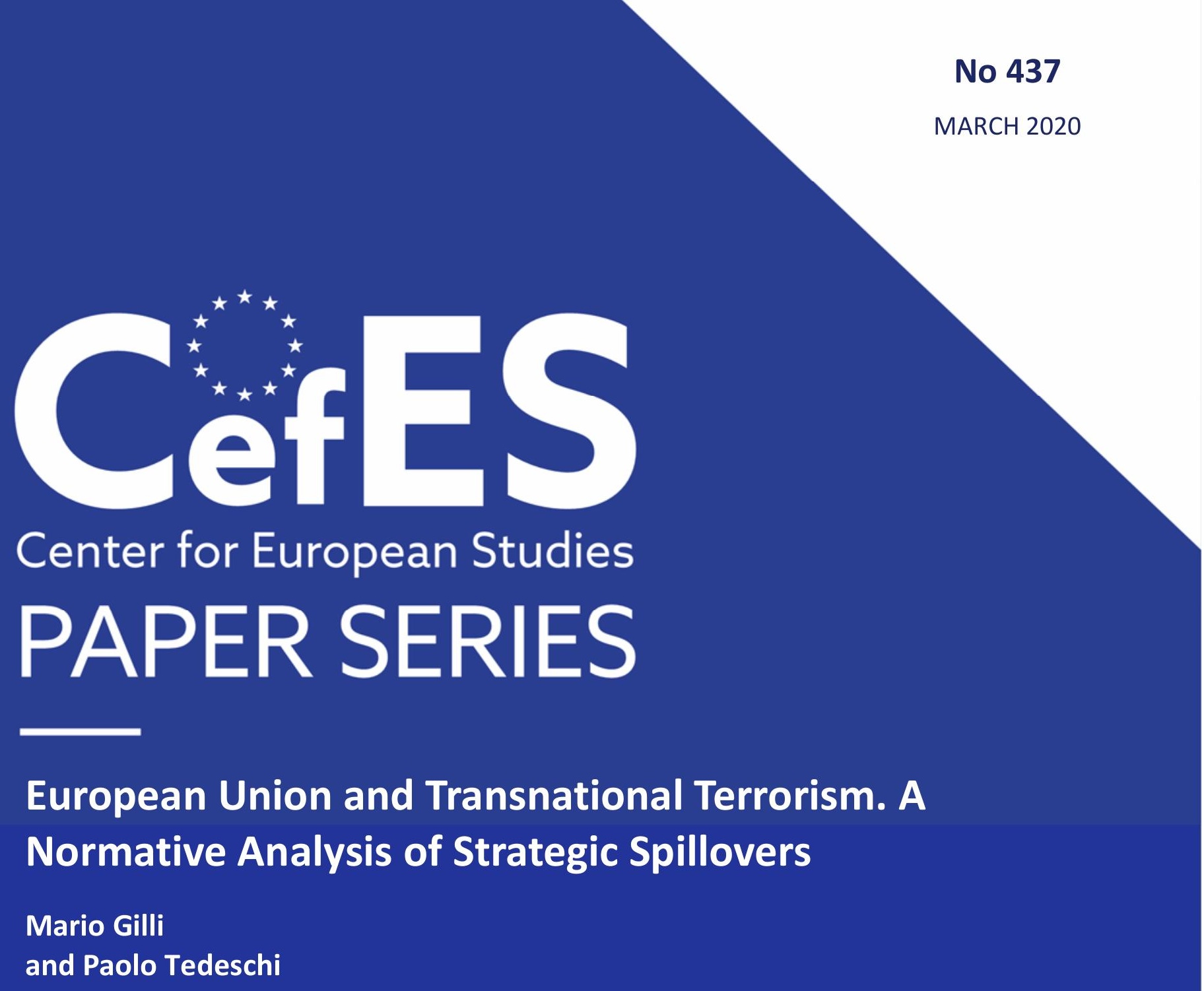




\title{
European Union and Transnational Terrorism. A Normative Analysis of Strategic Spillovers
}

\author{
Mario Gilli \\ Department of Economics. Management and Statistics and \\ Center for European Studies, University of Milan-Bicocca. \\ Paolo Tedeschi \\ Department of Economics. Management and Statistics and \\ Center for European Studies, University of Milan-Bicocca.
}

March, 2020*

\begin{abstract}
There are two main categories of antiterrorism policies - active and defensive. Active measures target the terrorists directly, and by weakening their ability to operate, are a sort of public good. On the other hand, defensive measures try to protect a potential target. Unilateral defensive measures can induce terrorists to substitute one target for another, in particular a foreign one. These positive and negative externalities of different antiterrorism measures raise the question of the best institutional setting for countries cooperation. This paper studies how in democratic countries public opinion and past intelligence proactive policies affect the efficiency of counterterrorism defensive policies according to different institutional scenarios regulating cooperation among countries. The aim of the paper is to study the consequences of four different scenarios on the choice of defensive policies: full decentralization, intelligence cooperation, unanimous political cooperation, such as the European Union for security policies, and full political union, the first best solution. The main result is that intelligence cooperation reduces the possibility of inefficient defensive policies more than unanimous political cooperation, a case of second best theory.
\end{abstract}

${ }^{*}$ We would like to thank the partecipants of the CefES Research Project "Norms and rules. Causes and effects of socio-demographic, economic and political dynamics inside and outside Europe", the participants to the 1st CefES Conference on European Studies, the participants to NESPUTT 2019, and the participants to PEDD2020. The comments by Thomas Apolte, Roger Congleton, Stefano Colombo, Giorgio Di Maio, Mario Ferrero, Simon Hug, Samuli Leppala, Marco Mantovani, and Martin Paldam have been very useful. 


\section{Contents}

\begin{tabular}{lll}
\hline & Introduction & 4
\end{tabular}

\begin{tabular}{|ll|}
\hline 2 & Transnational Terrorism and Anti-Terrorism in the European \\
\hline \hline Union & $\mathbf{7}$
\end{tabular}

$2.1 \quad$ Islamist Terrorism in the European Union . . . . . . . . . . 7

2.2 Security and Policy Governance in the European Union . . . . . 8

$\begin{array}{lll}3 & \text { The Basic Model } & 11\end{array}$

3.1 The Different Scenarios . . . . . . . . . . . . . . . . . . . . . . 14

3.1.1 Scenario 1: Full Decentralization . . . . . . . . . . . . 15

3.1 .2 Scenario 2: Intelligence Cooperation . . . . . . . . . . 15

3.1.3 Scenario 3: Unanimous Political Cooperation à la EU . . 17

3.1.4 Scenario 4: Full Political Union . . . . . . . . . . . . . . 18

3.2 Comments on the Model . . . . . . . . . . . . . . . . . . . . . . 19

4 Countries Efficient Policy Choices 20

4.1 The Countries Efficient Choices with Private Information . . . . 21

4.2 Efficient Choices when Countries Share the Information . . . . . 22

5 The Equilibrium Policy Choices 24

5.1 The Terrorists Sequential Rational Choices . . . . . . . . . . . 24

5.2 Perfect Bayesian Equilibria in Scenario 1 with Fully Decentral-

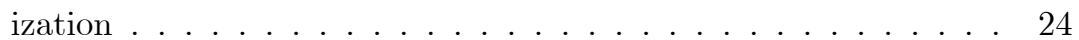

5.3 Perfect Bayesian Equilibria in Scenario 2 with Intelligence Coop-

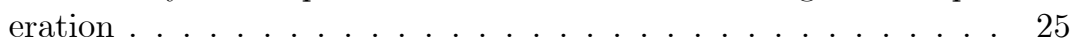

5.4 Perfect Bayesian Equilibria in Scenario 3 with Unanimous Policy

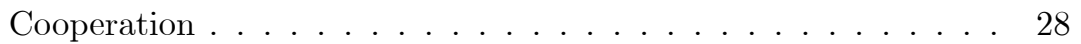

5.5 Remarks on the Equilibria in the Full Decentralization Scenario . 28

5.6 Remarks on the Equilibria in the Intelligence Cooperation Scenario 30

5.7 Remarks on the Equilibria in the Unanimous Political Union Sce-

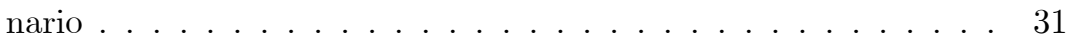

$\begin{array}{lll}6 & \text { Conclusion } & 32\end{array}$

\begin{tabular}{lll}
\hline I & Appendix & 38
\end{tabular}

7 Appendix A: Calculation of Terrorists' Sequential Rational Choices 38

8 Appendix B: Efficient Choices and Countries' Information 42

8.1 The Countries Efficient Choices with Private Information . . . . 42

8.2 The Countries Efficient Choices with Common Information . . . 45

9 Appendix C: Perfect Bayesian Equilibria with Full Decentralization 47 
10 Appendix D: Perfect Bayesian Equilibria with Intelligence Cooperation

68

11 Appendix E: Perfect Bayesian Equilibria of the Game in Scenario 3 with Policy Cooperation

102 


\section{Introduction}

Between 2010 and 2016, over 300 people were killed in terrorist attacks in the European Union. In particular, between January 7, 2015 and July 26, 2016, 239 persons were killed by Jihadist terrorists in France, including the 130 people murdered on November 13, 2015, in the attacks at Stade de France and at the Bataclan music hall, the worst single massacre of French civilians since the one committed by the Nazis in the village of Orandur-sur-Glane during Word War II. French was also the first victim of this new wave of Islamist terrorism, but Belgium too was struck in March 2016, with the suicide attacks on Brussels National airport and the Maelbeek metro station. Germany was attacked on 19 December 2016 by Jihadist Anis Amri driving a truck into a Berlin Christmas market. After 2016, deaths fell by 52 per cent, from 168 in 2016 to 81 in 2017. From January until October 2018, fewer than ten deaths because of Jihadist terrorism were recorded in Europe, however despite the fall in deaths, the number of incidents and arrests rose, in particular in 2019 there have been 16 foiled Jihadist terrorist plots in EU, witnessing the effectiveness of European anti-terrorism policies.

In an era where terrorism and other serious crime operate across borders, both the European Union and its Member States have a responsibility towards their citizens to deliver an area of internal security where individuals are protected, in full compliance with EU fundamental rights. Member States have the front line responsibility for security because they are democratically accountable to their citizens, but cannot address transnational threats effectively acting on their own. That is why it is crucial to consider the European governance in which national authorities can and do work together effectively to tackle shared challenges so that it possible to construct an effective Security Union in which the rights and freedoms of citizens are well protected.

The terrorists' attacks in Europe raise at least two different but related research topics: the analysis of the determinants of governments' counterterrorism reactions and the search for the most efficient institutional cooperation to reduce terrorism threats. Both aspects require both a theoretical and an empirical enquiry; in particular it is not possible to avoid a theoretical model because the observable equilibrium outcomes crucially depend on the non-observable out of equilibrium actions. The main purpose of this paper is to enlighten the combined effect of voters' propensity towards securization policies and of institutional scenarios on the (in)efficiency of counter-terrorism defensive policies, when active intelligence policies are inherited by a country's past history. In this way, we compare the relative efficiency of different institutional agreements as a function of voters' propensity for securization policies. To be clear, we are not investigating the best counter-terrorism policies, their costs and benefits and whether some anti-terrorism policies can be counter-productive. Instead, we take active policies as given, and we analyse how defensive policies efficiency is affected by governments' information and institutional cooperation. This analysis requires the combination of two different streams of literature, the works on political 
unions and the research on counterterrorism policies 1

Counterterrorism policies consist of government's actions aimed to reducing terrorists' motivation, to inhibit terrorist attacks or to curtail their consequences, and can be divided in two main categories, active and defensive. Active or offensive measures target the terrorists, their resources, or their supporters directly: by weakening the ability of terrorists to operate, active policies reduce the frequency and prevalence of attacks against all at-risk targets, home and foreign; hence they are a sort of public good. Defensive or passive policies try to protect a potential target against an attack or to ameliorate the damage in case of an attack. Defensive measures may involve the installation of technological barriers, the hardening of targets (e.g., barriers in public spaces), and the deployment of security personnel. Unilateral defensive measures can induce terrorists to substitute one target for another relatively cheaper, in particular a foreign one, hence globally they generate negative spillover effects. Moreover, offensive and defensive policies can be complementary, in the sense that active policies may provide information useful for defensive measures. On the other hand, offensive policies can backlash on the terrorists' attitude to attack a specific country since these actions may create new grievances. This distinctive emphasis on positive and negative externalities is by now standard, but significantly less works study their different public observability: many active policies, such as intelligence, are not publicly known, while defensive measures should be observable to be effective. We start from these aspects, assuming that intelligence activities is a country private information given by its past history, that active and defensive policies complement in reducing the likely effects of terrorists' attacks and that the terrorist organization targets more heavily a country that it believes is more heavily involved in active policies, using each country defensive policy to try to infer such information ${ }^{2}$ Moreover, since counter-terrorism policies affect the likelihood of terrorism attacks in different ways according to these different spillover effects, the literature on political union is crucial to analyze these problems. Given these characteristics of counter-terrorism policies, it should be clear the relevance of the institutional settings that trade-off the benefits of policies' coordination to internalize policy spillovers and the costs of uniform political decisions on otherwise heterogeneous citizens, i.e. the research program on political unions.

This paper investigates the interplay between the possibly cooperative decisions in defensive measures and the citizens propensity towards law and order policies, when two countries are threatened by the same terrorist group and defensive policies are influenced by given country specific preemptive measures. Specifically, we consider a peculiar type of active counter-terrorism measures, intelligence and covert missions that are country specific and that we assume depends on the country past history. We also assume that these active measure

\footnotetext{
${ }^{1}$ On political union see e.g. Alesina and Spolaore 1997 and 2006, Alesina et al. 2000 and 2005, on counterterrorism policies see e.g. Arce and Sandler 2005, 2007 and 2010, Bandyopadhyay and Sandler 2011, Brueck et al. 2008, Bueno de Mesquita 2007, Das and Lahiri 2006, Enders and Sandler 2006b.

${ }^{2}$ Kunreuther and Heal 2003; Sandier and Enders 2004 and 2006.
} 
have two opposite effects: on one hand they backfire in the sense that terrorists would prefer to attack countries more heavily involved in active policies even if terrorists are not fully informed on a country involvement in intelligence activities, on the other hand intelligence allows more effective defensive measures, reducing terrorism effectivity. Within this complex interaction between active and defensive policies, our institutional reference point is the European Union (EU) and its counter-terrorism governance. Thus, we compare four different institutional scenarios: full decentralization, intelligence coordination, political union such as EU, and full integration (efficient reference point). For each of these scenarios, we show the inefficiencies related to the interplay between active and defensive policies and lack of internalization of the strategic effects of such policies; moreover, we connect such inefficiencies to the countries' democratic accountability as a function of citizens' propensity towards law and order policies and on the institutional cooperation possibilities between countries.

The model aims to capture this strategic interaction between a transnational terrorist organization such as ISIS, with two democratic countries that are potential targets for terrorist attacks and that may coordinate their antiterrorism activities in different ways, depending on the institutional scenario. We consider a four-stage game where nature chooses the active policies, the two countries are fully or partially informed about nature's choices, and choose the defensive policies, then the terrorist organization decides which country to attack, and finally, observing the level of their country damages and the government public policy, voters decide whether to dismiss or not the incumbent government. We look for closed form solutions to make precise comparisons, thus we simplify the model assuming specific functional forms for the conflict technology and agents' payoffs; moreover we also assume a finite set of players' actions and of country types. Specifically, we suppose that each country is characterized by two types of active policies, high or low, and that given its private information on the amount of intelligence, each government must choose whether to allocate a high or low amount of the current public extra budget to defensive policies or to other public goods. Regarding players' payoffs, we assume that the citizens wish to trade off between lack of damages caused by terrorists' attacks and public expenditure on public goods different from defensive measures, while the government just wish to be reelected. As for the payoff of the terrorist organization, the definition of its ultimate objective is a complicated and debated issue. In this paper, we focus on its immediate goal, the amount of damages inflicted on a country, positively weighted by the involvement of that country in active antiterrorism policies (the backlash effect). This aspect is consistent with the empirical evidence that successful attacks stimulate the recruitment of terrorists, especially when target countries are actively involved in antiterrorism policies. This structure of strategic interaction lead to a non trivial signalling game, with four types, two senders - the governments of two countries -, one receiver - the terrorist organization -, and two non strategic players, each country voters.

The model is related but different from the established literature in several important aspects. First, as usual, it assumes that both the terrorist organi- 
zation and the countries act strategically. Second, each country government is democratically accountable to its citizens, that reelect or dismiss the incumbent government depending on their policy decisions. Third, active measures are a country private information, and this will influence its optimal defensive decisions because of complementarity between active and defensive policies. Moreover, all countries are potential targets for a terrorist attack, however the greater the amount of active measures, the higher terrorists' propensity to attack that country, a backlash effect. Since active policies are not observable by

terrorists', defensive measures may work as a signal. Finally, the possibility of defensive policy cooperation depend on four possible institutional scenarios, i.e. from four different structure of strategic interaction, from a full decentralized setting to a full political union, through the intermediate steps of simple intelligence cooperation and of unanimous policy cooperation, as in EU. Within this model, we show that the likelihood of inefficient policy choices has a complex non monotone relationship with the amount of institutional cooperation, unless public opinion is extreme for or against law and order policies. In particular, domestic policy choices are usually inefficient because of excessive expenditure on defensive policies.

The paper is organized as follows. Section 2 presents some information on Islamic terrorism and on EU anti-terrorism governance, section 3 presents and discuss the model, sections 4 and 5 present the efficient and the equilibrium policies in our institutional scenarios, section 6 compares efficient and actual outcomes and section 7 concludes. The calculations and the proofs are in the online Appendix: since the analysis of this four stage signaling model requires many tedious long calculations to characterize the set of possible equilibria, in the main text we only report the relevant results, together with self-explaining figures.

\section{Transnational Terrorism and Anti-Terrorism in the European Union}

\subsection{Islamist Terrorism in the European Union}

Since 1968, Europe has confronted an increasing threat of transnational terrorists, i.e. incidents involving perpetrators, victims, institutions, governments, or terrorists from two or more countries. After the 9/11 attacks, the EU set three guidelines for how European states should respond to terrorism

1. all the aspects of anti-terrorism policies should be under control of civil authorities;

2. the security forces must conduct all anti-terrorist operations within the law;

3. special powers, which may become necessary, should be approved by the legislature only for a fixed and limited time. 
However, until the 2004 bombing in Madrid, Al Qaeda did not select European countries for attack. The situation changed following the arrival of European troops in Afghanistan and in Iraq. The impact in terms of deaths and injured was shocking, in particular because of the 192 deaths and 2050 injuries of the 2004 Madrid train bombing and of the 52 deaths and 784 injuries because of 2005 London bombing. After some quiet years, in the last 10 years, there has been a new rise in Islamic Terrorism in Europe, in particular the years 201416 saw more people killed by Islamic terrorist attacks in Europe than all ten previous years combined, and the highest rate of attack plots per year. Since 2014, more than 20 fatal attacks have been carried out in Europe. France saw eight attacks between January 2015 and July 2016; United Kingdom saw three major attacks carried out in a span of four months in early 2017; other targets in Europe have included Belgium, Germany, Russia, and Spain. The transcontinental city of Istanbul also saw both bombings and shootings, in January 2016, June 2016 and January 2017. The deadliest attacks of this period have been the November 2015 Paris attacks (130 killed), the July 2016 Nice truck attack (86 killed), the June 2016 Atatürk Airport attack (45 killed), the March 2016 Brussels bombings (32 killed), and the May 2017 Manchester Arena bombing (23 killed). Most attacks since 2014 have been carried out by individuals using guns, knives and vehicles. A notable exception is the Brussels cell, which carried out two of the deadliest attacks of the period. Despite high-profile attacks in Barcelona and Manchester in 2017, the surge in terrorism over the past few years has abated. France recorded seven deaths from terrorism in 2017, down from 162 in 2015. Germany experienced 26 deaths in 2016, but just one in 2017. There was also a large improvement in Belgium, where the number of deaths fell from 36 in 2016 to 2 in 2017. The deadliest terror attacks in 2017 were a suicide bombing in the United Kingdom at an Ariana Grande concert in Manchester, that killed 23 people and injured an additional 119 and a vehicular attack in Barcelona that killed 23 people and injured 101 more. Decreased activity from ISIS and other Jihadist groups in Europe has occurred alongside significantly stricter counterterrorism and security measures throughout Europe and loss of territory in Iraq and Syria, in particular increased counter-terrorism spending and security measures have reduced the frequency and the lethality of attacks, as witnessed by the increased number of incidents and of arrests ${ }^{3}$ From the data of the Europol EU Terrorism Situation and Trend Report (TE-SAT) we can construct the following graphs of deaths, injured and arrests in EU from 2008 to 2017 because of Islamist Terrorism 4

\subsection{Security and Policy Governance in the European Union}

Our main institutional reference point is the European Union (EU) and its antiterrorism governance structure. In the simplest of terms, EU is more than an

\footnotetext{
${ }^{3}$ Data from the Global Terrorism Index.

${ }^{4}$ The official definition of "Islamist Terrorism" by European Union (Europol (2009-2018) is "terrorism ... perpetrated by individuals, groups, networks or organizations which evoke Islam to justify their actions".
} 


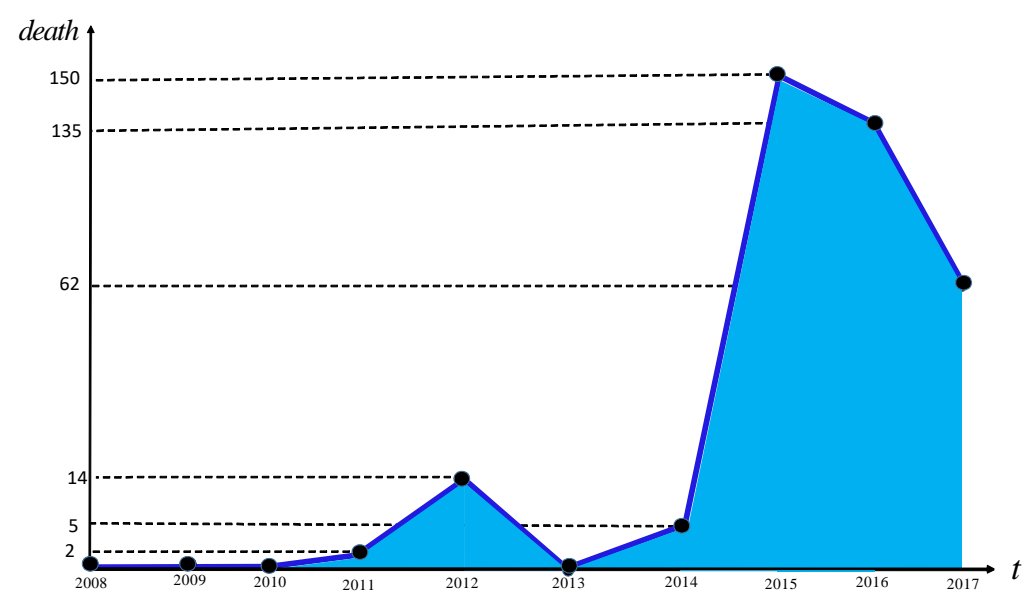

Figure 1: Deaths in EU because of Islamist Terrorism

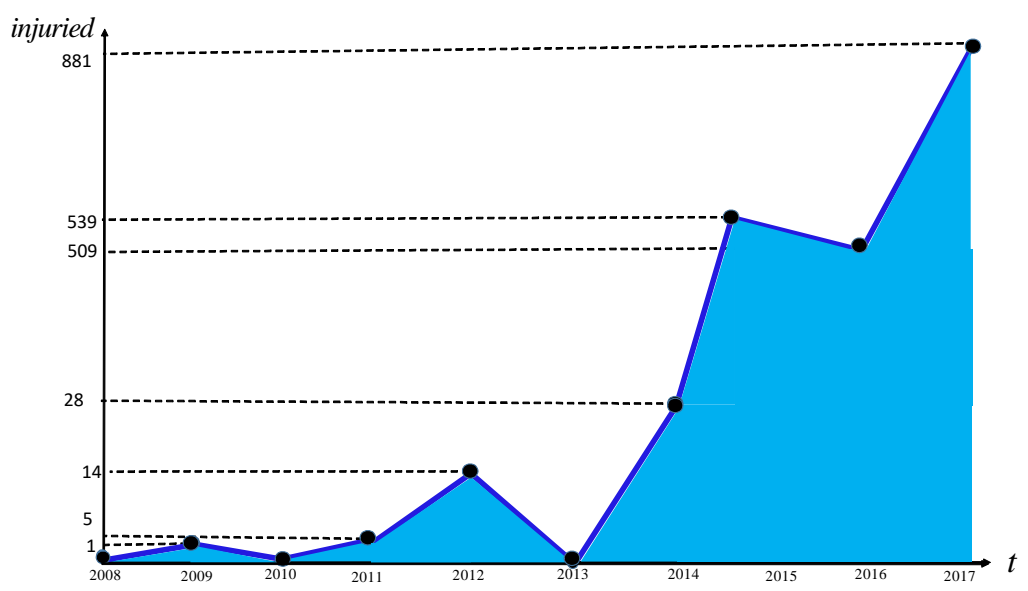

Figure 2: Injuried in EU because of Islamist Terrorism 


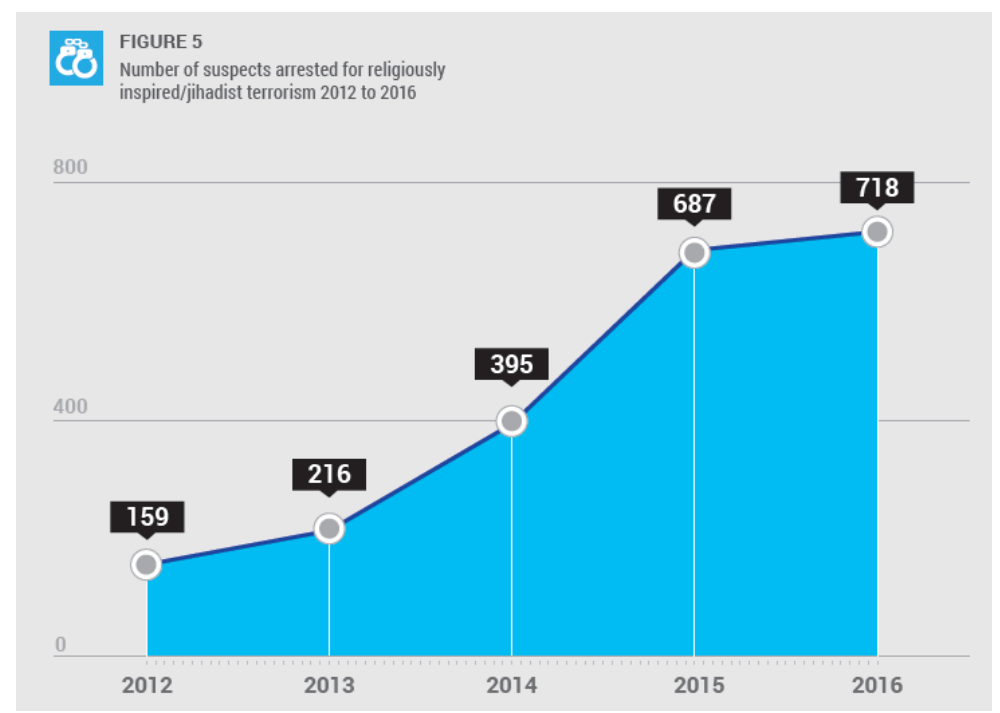

Figure 3: Arrests in EU because of Islamist Terrorism

international organization, but less than a federal state, with a new and original form of governance. Its aim is to deal with transnational problems involving positive and negative externalities, in particular concerning economy, environment and security. The main EU challenge is to manage national differences providing a collective return in exchange of the limitation of national sovereignty. Within EU policies, the Area of Freedom, Security and Justice (AFSJ) is a collection of home affairs and justice policies designed to ensure security, rights and free movement within EU. Areas covered include the harmonization of private international law, extradition arrangements between member states, policies on internal and external border controls, common travel visa, immigration and asylum policies and police and judicial cooperation.

The "EU Global Strategy for Foreign and Security Policy" (hereafter EUGS) was presented on 28 June 2016 and represented the final result of a two yearlong reflection about the regional and global strategic landscape surrounding Europe, substituting the 2003 European Security Strategy. The title of the document, "Shared Vision, Common Action: A Stronger Europe", illustrates that EU governance considers very important to improve the sharing of aims and information, and the realization of common efforts involving all EU's members and their partners (beginning with the NATO). EUGS calls for a new EU approach to conflicts and crises as well as for new better links between EU internal and external policies, as required by the growth of the migration and terrorism phenomena. It establishes the improvement of the Common Foreign and Security Policy (CFSP) and of the Common Security and Defence Policy (CSDP). The EUGS confirmed that member States remained sovereign in their 
defence decisions: nevertheless, it also indicated that "to acquire and maintain many of these capabilities, defence cooperation must become the norm" and that "to increase investment in and solidarity on counter-terrorism are key". So the EUGS "will therefore encourage greater information sharing and intelligence cooperation between Member States and EU agencies. This entails shared alerts on violent extremism, terrorist networks and foreign terrorist fighters, as well as monitoring and removing unlawful content from the media. Alongside, the EU will support the swift recovery of Members States in the event of attacks through enhanced efforts on security of supply, the protection of critical infrastructure, and strengthening the voluntary framework for cyber crisis management". Improved information-sharing between CSDP and Justice and Home Affairs actors has been given particular prominence, with progress made in developing a pilot project for a Crime Information Cell located within a CSDP operation (EUNAVFOR MED Operation Sophia) to test integration of internal and external instruments at field level more systematically.

Besides this EU general scheme, new restrictive anti-terrorism rules were established by single countries: e.g. in Belgium the legislation on privacy intrusive techniques was broadly reviewed and modernized, including issues such hacking by the police into a laptop or a smartphone from outside, or infiltrating groups on internet. It became possible to search a house at night, in specific circumstances. Besides, the period of "garde à vue" was extended and new offences were added to the code of criminal law, including the act of preparing a terrorist attack. Finally the relationship between intelligence services and the judicial investigation became much more flexible than it was before. A database was set up where intelligence and police agencies share information about certain categories of people. It includes all sorts of extremism, not only Islamic extremism and people who were in Syria, but also far right extremism. Other actors such as prisons or probation officers were granted access to the database and are required to feed it.

\section{The Basic Model}

The aim of the model is to study the interplay between preemptive and defensive countermeasures against terrorism when two countries are threatened by the same terrorist group and the national governments are democratically accountable to citizens. Our reference point is the European Union and its institutional counter-terrorism governance, previously explained. European countries have a long and specific history of involvement in terrorism related situations, often related to their post-colonial heritage, as the case of Jihadist terrorism shows. Because of this characteristic, we consider a peculiar type of active counterterrorism measure, intelligence and covert missions, that we assume as given and as country specific, i.e. correlated to each country past history. Moreover, these given active measures have two opposite effects: on one hand they backfire in the sense that terrorists would prefer to attack countries they believe to be more heavily involved in active policies, on the other hand they allow more ef- 
fective defensive measures, reducing terrorism effectivity. Finally, an important political characteristic of European countries is that they are democratic, thus it is important to model each government accountability towards each country specific voters.

We formalize these ideas as follows. We consider five agents: the governments of country $j \in\{A, B\}, G^{j}$, a terrorist organization $T$ and the voters of country $j \in\{A, B\}, V^{j}$. Country $j$ is characterized by a given amount of active policies $p^{j}$, which is its private information. Each government, $G^{j}$, receives a signal $\sigma^{j} \in\left\{p^{j},\left(p^{A}, p^{B}\right)\right\}$ that provides different information on countries' intelligence, according to the institutional setting: when the country are independent $\sigma^{j}=p^{j}$, i.e., each country knows its intelligence only, when there is information sharing $\sigma^{j}=\left(p^{A}, p^{B}\right)$, i.e. each country knows its and the other country amount of active policy. Then each government chooses the allocation of the public budget between defence measures $d^{j}$ and residual public expenditure $g^{j}$. Hence $1=g^{j}+d^{j}$, because of budget constraint. The governments' decisions are jointly taken when there is policy cooperation, otherwise they are taken independently and simultaneously. The terrorist organization $T$ observes $\left(d^{A}, d^{B}\right)$ but not countries intelligence policies, and decides the allocation of available resources between attacks to country $A$ and country $B, a^{A}$ and $a^{B}$, so that $a^{A}+a^{B}=1$, again because of budget constraint. Finally, voters of each coun$\operatorname{try} j, V^{j}$, decide whether to remove or not the government, $R=1$ or $R=0$. As far as the agents' objectives, we assume that each government $G^{j}$ is interested in maximizing the probability of re-election, hence $U^{G^{j}}\left(R^{j}=0\right)>U^{G^{j}}\left(R^{j}=1\right)$, while the terrorist organization $T$ is interested in maximizing the weighted total loss from attacks $E^{T}\left(p^{A} \mid d^{A}\right) l^{A}+E^{T}\left(p^{B} \mid d^{B}\right) l^{B}$, where $l^{j} \in[0,1]$ is the intensity of losses measured in percentages, and the weight $E^{T}\left(p^{j} \mid d^{j}\right)$ is the terrorist evaluation of government $j$ active policy, given terrorists' information. The idea is that the terrorists organization prefers to attack countries that have more strong active policies. This is consistent with the empirical evidence 5 and formalizes the idea of active policies backlash. Finally, we assume that the probability of removing the government is determined by probabilistic voting à la Persson-Tabellini, so that voters are not strategic players. Let we stress that this version of governments' budget constraints, means that terrorists' attacks do not affect the available resources. Similarly, we assume that the terrorists' budget constraint is not affected by countries' active or defensive policies, coherently with the situation we want to model.

Summing up, the timing of the play is the following:

1. Nature characterize each government $G^{j}$ by a given stock of active policies, $p^{j}$ and by a signal $\sigma^{j} \in\left\{p^{j},\left(p^{A}, p^{B}\right)\right\} ;$

2. $G^{A}$ and $G^{B}$ simultaneously decide how much of the current budget should be allocated to defensive policies $d^{A}$ and $d^{B}$;

3. $T$ does not observe $\left(p^{A}, p^{B}\right)$, just $\left(d^{A}, d^{B}\right)$, then decides how much resources to use to attack countries $A$ and $B, a^{A}$ and $a^{B}$ causing a loss of

\footnotetext{
${ }^{5}$ E.g. Sandler 2003.
} 


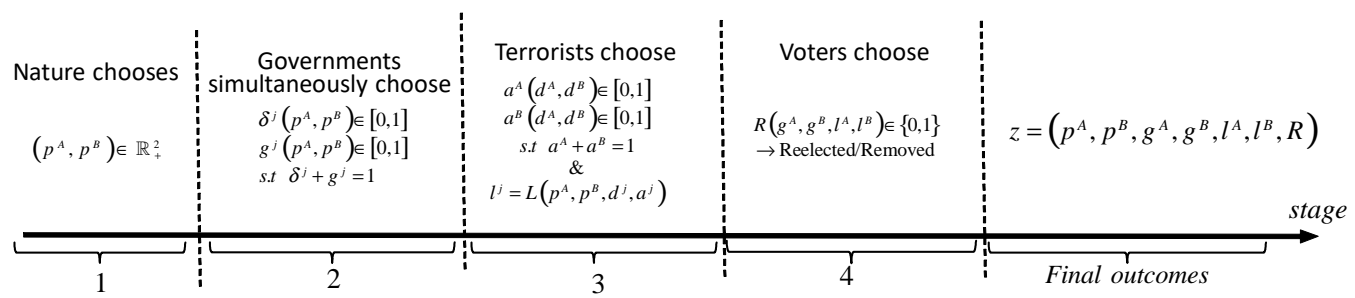

Figure 4: The timing of the game

intensity of $l^{A}$ and $l^{B}$

4. Voters of country $j, V^{j}, j \in\{A, B\}$, remove or not the government, $R=1$ and $R=0$, according to the probabilistic voting model à la PerssonTabellini as a function of $\left(d^{A}, d^{B}, l^{A}, l^{B}\right)$.

The timing is constructed to model the idea that intelligence policies depend on past choices that can't be renegotiated, however they affect the effectivity of subsequent strategic choices on defensive policies. Moreover, by definition terrorist can't observe countries' intelligence, but can extract information from countries' strategic choices. Finally, voters observe the effects of government's choices and decide whether to dismiss it or not. The timing of the games is represented in figure 1.

To simplify calculations and to derive closed form solutions, we consider the following specific assumptions on variables and on functional forms:

1. The countries' possible types are finite, in particular:

$$
\left(p^{A}, p^{B}\right) \in\{1,2\} \times\{1,2\}
$$

where $p^{j}=1$ means weak active policy, $p^{j}=2$ strong active policy;

2. Types are independent and symmetric, hence

$$
\operatorname{Pr}\left\{p^{j}=1 \mid p^{-j}=1\right\}=\operatorname{Pr}\left\{p^{j}=1\right\}=\pi ;
$$

3. The government's possible choices of defensive policies are finite, in particular

$$
\left(d^{A}, d^{B}\right) \in\{0,1\} \times\{0,1\}
$$

where $d^{j}=0$ means weak defensive policy, $d^{j}=1$ strong defensive policy; 
4. Country $j$ loss from a terrorist attack is given by the following ratio

$$
l^{j}=L^{j}\left(a^{j}, p^{A}+p^{B}, p^{j}, d^{j}\right)=\frac{a^{j}}{p^{A}+p^{B}+p^{j} d^{j}}
$$

that models in the simplest way the public good characteristic of active policies and the complementarity between active and defensive policies. This functional form is a version of the standard Tullock conflict technology ${ }^{6}$ adapted to this contest;

5. The probability of not removing government $j$ depends on a parameter $\omega$ uniformly distributed on $[-1, \gamma]$ so that government $j$ is re-elected if and only if $\omega \leq \gamma g^{j}-l^{j}$, hence

$$
\operatorname{Pr}\left\{R^{j}=0 \mid g^{j}, l^{j}\right\}=\frac{1}{1+\gamma}\left(1+\gamma g^{j}-l^{j}\right)=1-\frac{1}{1+\gamma}\left(\gamma d^{j}+l^{j}\right)
$$

where $\gamma \in[0,1]$ is a parameter that reduces the effect of the bundle of public goods different from security, to account for the fact that there many different public goods apart from security and that usually the voters' preferences are dispersed on generic public goods while they agree on security. In other words, $\gamma$ is a measure of the weight the voters attach to tangible and intangible public goods different from defensive policies. Hence the greater $\gamma$, the smaller the voters' weight on security policies.

These assumptions are obviously restrictive, however, besides allowing the calculations of closed form solutions, they are also used to sign the global effects of opposing externalities. Their meaning is quite transparent. The loss from a terrorist attack $l^{j}$ is modelled adapting to this setting the standard Tullock conflict technology, including also the idea of strategic complementarity between intelligence and defensive policies, so that $l^{j}$ is increasing in the magnitude of the attack $a^{j}$, decreasing in the total amount of active policies $p^{A}+p^{B}$, that thus work as a public good, and decreasing in defensive measures $d^{j}$ interacted with the amount of active policy $p^{j}$ to model the idea that defensive and active policies are complements. Type $p^{j} \in\{1,2\}$ as well as $d^{j} \in\{0,1\}$ are an effective way of modelling the idea that active and defensive policies might be weak or strong, avoiding discontinuity in the conflict technology. Finally, the model of probability voting is a standard version à la Persson-Tabellini where the probability of re-election is increasing in the amount of the public good $g^{j}$, decreasing in the loss from a terrorist attack $l^{j}$, and the greater $\gamma$, the smaller the voters' weight on security policies.

\subsection{The Different Scenarios}

We consider four possible institutional scenarios that correspond to four different game's forms.

\footnotetext{
${ }^{6}$ Tullock 1980.
} 


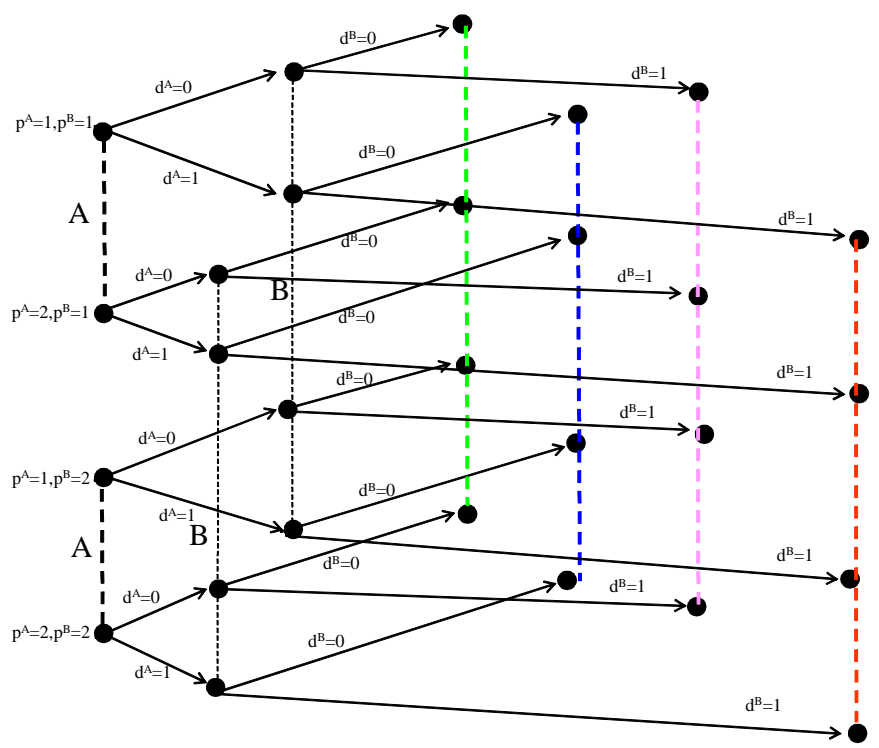

Figure 5: Full decentralization

\subsubsection{Scenario 1: Full Decentralization}

In a full decentralized environment, countries share neither information nor policy choices. This scenario is formalized assuming that a country's signal provides no information on the other country stock of active policy, hence

$$
\sigma^{j}\left(p^{A}, p^{B}\right)=p^{j} .
$$

Hence, each country choice maps from the set of its possible active policies, i.e. country $j$ private information, to the set of defensive policies:

$$
\delta^{j}:\{1,2\} \rightarrow\{0,1\} .
$$

The first stage of the game tree with full decentralization is represented in the following figure.

\subsubsection{Scenario 2: Intelligence Cooperation}

In an environment with intelligence cooperation, the countries share information on past active policies. This scenario is formalized assuming that a country's signal provides full information on the joint stock of active policies, so that:

$$
\sigma^{j}\left(p^{A}, p^{B}\right)=\left(p^{A}, p^{B}\right) .
$$




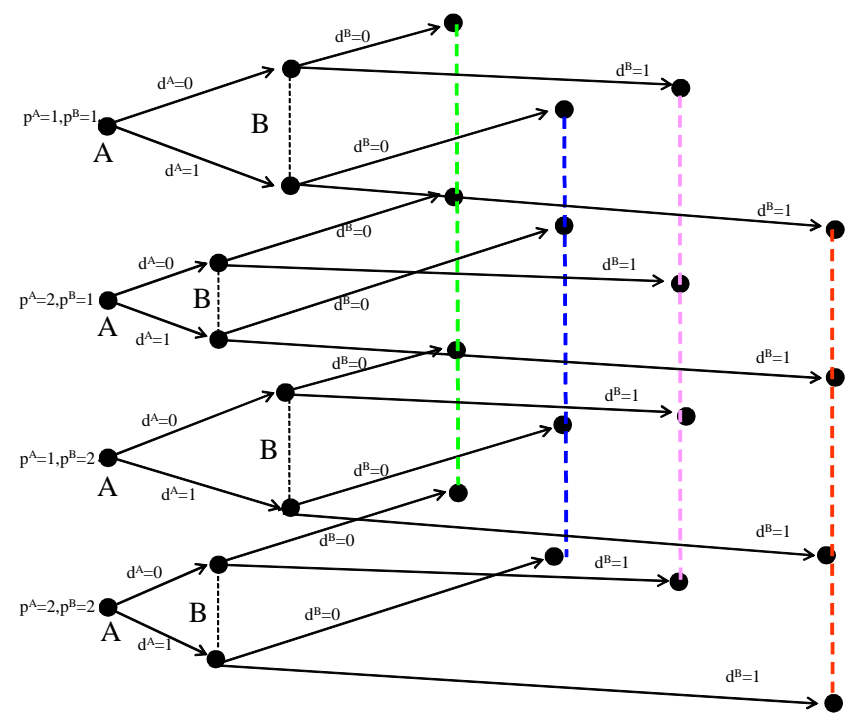

Figure 6: Intelligence cooperation

Hence, each country choice maps from the set of both countries possible active policies to the set of defensive policies of country $j$, so that:

$$
\delta^{j}:\{1,2\} \times\{1,2\} \rightarrow\{0,1\} .
$$

In this institutional scenario, there is perfect sharing of information on active policies between countries, however each government independently implements its defensive policies to maximize the probability of re-election. The first stage of the game tree with intelligence cooperation is represented in the following figure.

The importance of intelligence cooperation emerges by the reconstruction of ISIS last terrorists attacks in Europe, where some of them could have been avoided if intelligence information would have been fully shared within European countries ${ }^{7}$ Existing information sharing systems provide a vital operational tool for European countries, but there is agreement that there are gaps in functionality, coverage and inter operability. This means that there is scope for greater and better information sharing between member states, and a key facilitator should the building of trust and awareness of the benefits of sharing information. In this paper we do not tackle the issue of trust building, however we hope that our results will consolidate the global view on the benefits of sharing all the intelligence information.

\footnotetext{
${ }^{7}$ This has been the case for the Paris 2015 and the Berlin 2016 attacks.
} 


\subsubsection{Scenario 3: Unanimous Political Cooperation à la EU}

In an environment with policy cooperation, the countries share information on active policies and jointly choose the defensive policies. This scenario is formalized assuming that a country's signal provides full information on the joint stock of active policies:

$$
\sigma^{A \cup B}\left(p^{A}, p^{B}\right)=\left(p^{A}, p^{B}\right)
$$

so that the countries' strategies are the following kind of maps

$$
\delta^{A \cup B}:\{1,2\} \times\{1,2\} \rightarrow\{0,1\} \times\{0,1\} .
$$

In this institutional scenario, there is perfect sharing of information on active policies between countries, the problem is how to model the payoff function of a Political Union such as EU. There are two main approaches to study organizations regulated by formal voting procedures. The first approach models a given institutional scenario with as many details as possible while retaining technical feasibility. We are not following this approach, even if we recognize its importance. The alternative approach, the one used in this paper, aims at studying general issues and their impact on the equilibria of an international organization such as EU, constructing a very simple model, artificial perhaps, that isolates as much as possible the issues under analysis, analyzing the effects on equilibrium outcomes. As explained in section 4, EU's foreign and security policy decision are governed according to EUGS, which requires unanimity. The literature that may be used to model the policies choice process in this institutional scenario, is huge and diversified, from the analysis of veto power in voting model 88 to the study of the consequences of unanimous jury verdicts 9 from the calculations of the equilibria in delegated games with unanimity ${ }^{10}$ to the general analysis of decision making in committees ${ }^{11}$ from the study of social choices in unanimity games ${ }^{12}$ to the works on Bayesian Persuasion ${ }^{13}$ from the analysis of strategic delegation and voting rules ${ }^{14}$ to the effect of coalition formation in bargaining models ${ }^{15}$ However, all these studies consider detailed models of strategic interaction, while we need a concise tool to model decision making in the unanimity EU setting. Moreover, in many of the above studies the focus is either the role of incomplete information or the search for efficient solutions, an approach that by construction we exclude from our institutional scenario. Our starting idea is that a government would approve a collective decision making only if the decision would not reduce its wellbeing. This implies that there is no substitutability between countries payoffs, hence the union payoff function

\footnotetext{
${ }^{8}$ See e.g.Tsebelis 2002.

${ }^{9}$ See e.g. Feddersen and Pesendorfer 1998.

${ }^{10}$ See Granoty et al. 2003.

${ }^{11}$ Levy 2007.

${ }^{12}$ Kalai and Samet 1985.

${ }^{13}$ Gentzkow and Kamenica 2011.

${ }^{14}$ Harstad 2010.

${ }^{15}$ Rogna 2019.
} 
should satisfy the following restriction:

$$
U^{G^{A \cup B}}\left(U^{G^{A}}\left(R^{A}\right), U^{G^{B}}\left(R^{B}\right)\right)=\min \left\{U^{G^{A}}\left(R^{A}\right), U^{G^{B}}\left(R^{B}\right)\right\},
$$

i.e. we use the Rawlsian social welfare function as a simple way of modelling political decision that satisfy the above institutional conditions. It means that we model the EU unanimous decision making on security matter as if the union considers the governments' welfare as perfect complements. The point is that the governments take a collective decision, however each government is accountable to its own electorate, and this synthetic functional form is a way to model both these aspects, collective decision making and independent accountability. Moreover, to reduce the enormous number of possible equilibria, we limit our analysis to symmetric equilibria because in this specific institutional setting we consider unrealistic that negotiations between symmetric players may lead to asymmetric agreements ${ }^{16}$

\subsubsection{Scenario 4: Full Political Union}

In an environment with full political union, de facto there is a unique country, so there is perfect information on active policies and the defensive policies is chosen by a single government. This scenario is formalized as follows:

$$
\begin{aligned}
\sigma^{A B}\left(p^{A}, p^{B}\right) & =\left(p^{A}, p^{B}\right) \\
\delta^{A B}:\{1,2\} \times\{1,2\} & \rightarrow\{0,1\} \times\{0,1\} .
\end{aligned}
$$

Besides perfect sharing of information on active policies and joint choice of the defensive policies, in a full political union the aim is to maximize the global probability of re-election. This means that the political union payoff function is

$$
U^{A B}(R)=U^{G^{A}}(R)+U^{G^{B}}(R),
$$

while there is unique electorate with payoff

$$
U^{V}\left(g^{A}+g^{B}, l^{A}+l^{B}\right) .
$$

Clearly, between scenarios 3 and 4 there is no difference in the game form, the crucial difference regards the choice of defensive policies, that in case of unanimous policy cooperation are jointly chosen by two independently accountable agents, the governments of country A and country B, while in scenario 4 they are chosen by a single agent, accountable to the voters' of both countries. The first stage of the game tree with policy cooperation or with full political union is however identical and it is represented in the following figure.

The following table sum up the four different scenarios and their peculiarities:

\footnotetext{
${ }^{16}$ See Kalai and Samet 1985. For a discussion on asymmetric equilibria in symmetric games see Amir et al. 2010 and Cavalli et al. 2017.
} 


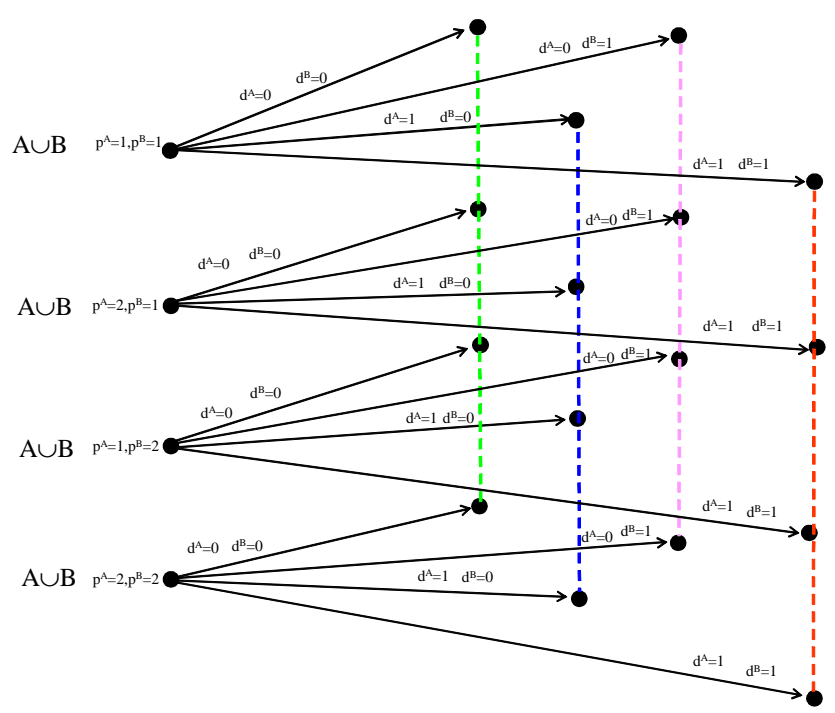

Figure 7: Political cooperation

\begin{tabular}{|c|c|}
\hline \multicolumn{2}{|r|}{ Table 1} \\
\hline Scenario & Changes in the game \\
\hline Fully decentralization & $\left\{\begin{array}{c}\sigma^{j}\left(p^{A}, p^{B}\right)=p^{j} \\
\delta^{j}:\{1,2\} \rightarrow\{0,1\}\end{array}\right.$ \\
\hline Intelligence cooperation & $\left\{\begin{array}{c}\sigma^{j}\left(p^{A}, p^{B}\right)=\left(p^{A}, p^{B}\right) \\
\delta^{j}:\{1,2\} \times\{1,2\} \rightarrow\{0,1\}\end{array}\right.$ \\
\hline Unanimous Political Cooperation & $\begin{array}{c}\sigma^{A \cup B}\left(p^{A}, p^{B}\right)=\left(p^{A}, p^{B}\right) \\
\delta^{A \cup B}:\{1,2\} \times\{1,2\} \rightarrow\{0,1\} \times\{0,1\} \\
U^{G^{A \cup B}}\left(U^{G^{A}}\left(R^{A}\right), U^{G^{B}}\left(R^{B}\right)\right)= \\
=\min \left\{U^{G^{A}}\left(R^{A}\right), U^{G^{B}}\left(R^{B}\right)\right\}\end{array}$ \\
\hline Full political union & $\begin{array}{c}\sigma^{A B}\left(p^{A}, p^{B}\right)=\left(p^{A}, p^{B}\right) \\
\delta^{A B}:\{1,2\} \times\{1,2\} \rightarrow\{0,1\} \times\{0,1\} \\
U^{A B}(R)=U^{G^{A}}(R)+U^{G^{B}}(R) \\
U^{V}\left(g^{A}+g^{B}, l^{A}+l^{B}\right)\end{array}$ \\
\hline
\end{tabular}

\subsection{Comments on the Model}

The aim of the model is to study the interplay between preemptive and defensive measures against terrorism when countries are threatened by the same terrorist group and the national governments are democratically accountable to citizens. The outcomes of this situation of strategic interaction depend on the institutional setting, i.e. whether there is fully decentralization, intelligence coordination, policy coordination or full political union. The crucial exogenous 
variables, are

- the probability of low active policies $\pi$, so that $1-\pi$ is a measure of a country historical involvement in foreign interventionism;

- the parameter $\gamma$ that reduces the weight on the probability of re-election of the bundle of public goods different from security, so that $1-\gamma$ is a measure of voters' propensity towards securization policy;

- the extensive form of the game, i.e. the institutional scenario.

In this way, the model is able to explain the amount of resources each government allocates to defensive counterterrorism and to other policies, the intensity of terrorism activity against each country, the domestic political stability and the efficiency of different institutional settings as functions of government active policies, of voters' concern for security and of the kind of cooperation among countries. The following table, sum up exogenous and endogenous variables, and their interpretation:

\begin{tabular}{|c|c||c|c|}
\hline \multicolumn{5}{|c|}{ Table 2 } \\
\hline Exog. var. & Interpretation & Endog. var. & Interpretation \\
\hline \multirow{2}{*}{$1-\pi$} & historical involvement in & $d^{j} \in\{0,1\}$ & defensive counterterrorism \\
& foreign interventionism & $j \in\{A, B\}$ & policies of country $j$ \\
\hline \multirow{2}{*}{$1-\gamma$} & voters' propensity towards & $a^{j} \in[0,1]$ & terrorists' attacks \\
& securization policy & $j \in\{A, B\}$ & towards country $j$ \\
\hline \multirow{2}{*}{$\Gamma$} & institutional scenario $\Leftrightarrow$ & \multirow{2}{*}{$d^{j *}(\Gamma) \gtrless d^{j E}(\Gamma)$} & $\begin{array}{c}\text { efficiency ranking as a } \\
\text { function of the scenario }\end{array}$ \\
\hline
\end{tabular}

In the following sections, we propose a qualitative view of our formal results, that are quantitatively specified in the Appendix.

\section{Countries Efficient Policy Choices}

Agents' efficient choices depend obviously on their private information, thus we have to distinguish between the two cases, when the countries have private information on their active policies and when they share this information. The efficient outcome for voters, then it is given by the choice $\delta^{E}\left(\sigma^{j}\left(p^{j}, p^{-j}\right)\right)$ that maximizes the probability of both government reelection. Thus, the social welfare function $\widehat{W}$ to maximize is

$$
\begin{gathered}
\widehat{W}=E\left[\operatorname{Pr}\left\{R^{A}=0 ; g^{A}, l^{A} \mid \sigma^{A}\right\}\right]+E\left[\operatorname{Pr}\left\{R^{B}=0 ; g^{B}, l^{B} \mid \sigma^{B}\right\}\right]= \\
=2-\frac{1}{1+\gamma}\left\{E\left[\gamma d^{A}+\frac{a^{A}}{p^{A}+p^{B}+p^{A} d^{A}} \mid \sigma^{A}\right]+E\left[\gamma d^{B}+\frac{a^{B}}{p^{A}+p^{B}+p^{B} d^{B}} \mid \sigma^{B}\right]\right\} \propto
\end{gathered}
$$




$$
\propto-E\left[\gamma d^{A}+\frac{a}{p^{A}+p^{B}+p^{A} d^{A}} \mid \sigma^{A}\right]+E\left[\gamma d^{B}+\frac{1-a}{p^{A}+p^{B}+p^{B} d^{B}} \mid \sigma^{B}\right] .
$$

Obviously the social welfare function depends also on the values of $a$, that is anticipated as sequentially rational.

\subsection{The Countries Efficient Choices with Private Informa- tion}

When there is full decentralization, then $\sigma^{j}\left(p^{j}, p^{-j}\right)=p^{j}$. The efficient outcome for the government and the citizens of country $j$ should consider this information limitation. Then the efficient choices are the solution of the following minimization problem

$$
\begin{gathered}
d^{E}=\left(d^{E, A}\left(p^{A}\right), d^{E, B}\left(p^{B}\right)\right) \in \arg \min E\left[W \mid \sigma^{j}\left(p^{j}, p^{-j}\right)=p^{j}\right] \Leftrightarrow \\
\Leftrightarrow d^{E}=\left(d^{E, A}\left(p^{A}\right), d^{E, B}\left(p^{B}\right)\right) \in \\
\in \arg \min \left\{E\left[\gamma d^{A}\left(p^{A}\right)+\frac{a^{S R} \pi}{p^{A}+p^{A} d^{A}\left(p^{A}\right)+1}+\frac{a^{S R}(1-\pi)}{p^{A}+p^{A} d^{A}\left(p^{A}\right)+2}\right]+\right. \\
\left.+E\left[\gamma d^{B}\left(p^{B}\right)+\frac{\left(1-a^{S R}\right) \pi}{p^{B}+p^{B} d^{B}\left(p^{B}\right)+1}+\frac{\left(1-a^{S R}\right)(1-\pi)}{p^{B}+p^{B} d^{B}\left(p^{B}\right)+2}\right]\right\}
\end{gathered}
$$

where the expectation is taken with respect to the possible states of nature $\left(p^{A}, p^{B}\right) \in\{1,2\} \times\{1,2\}$. In Appendix A we derived the following characterization of the efficient counter-terrorism policies.

Proposition 1 When each country has private information on its active policies, the efficient counter-terrorism defensive policies depend on the country historical involvement in foreign intervention $(1-\pi)$ and on voters' propensity towards securization policies $(1-\gamma)$. In particular

1. when both the country historical involvement in foreign intervention (1- $\pi$ ) is low and voters' propensity towards securization policies is high (low $\gamma$ ), efficient defensive policies are uniformly strong;

2. when the country historical involvement in foreign intervention is low/intermediate and voters' propensity towards securization policies is high/intermediate, efficient defensive policies are strong when active counterterrorism policies are significant and weak otherwise;

3. when voters' propensity towards securization policies is low/intermediate, efficient defensive policies are uniformly weak for any country historical involvement in foreign intervention.

Graphically, we get the following picture for the region $(\gamma, \pi)$ : 


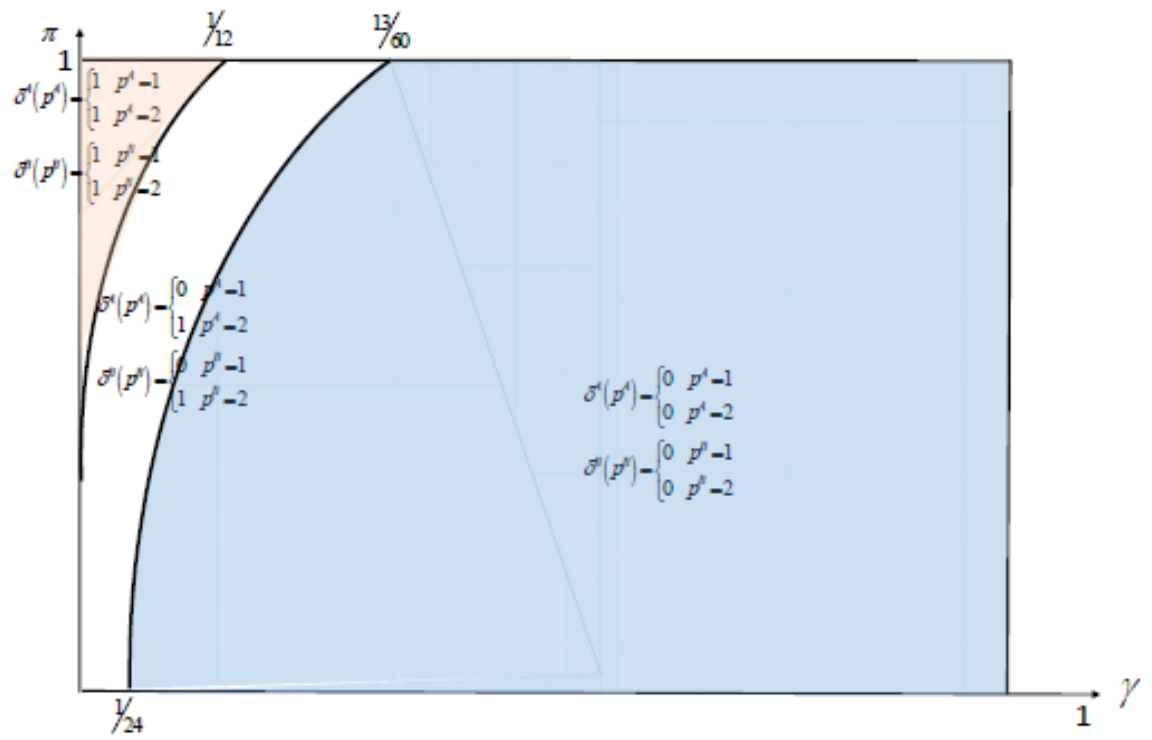

Figure 8: Countries' efficient policies with private information

Let us remark that efficiency requires that stronger defensive policies are associated to strong voters' request for securization policies (low $\gamma$ ), however this is not enough, it also requires a significant lack of historical involvement in foreign interventionism, i.e. high $\pi$, however for low/intermediate level of voters' request for securization policies, historical involvement in foreign interventionism is irrelevant.

\subsection{Efficient Choices when Countries Share the Informa- tion}

When the countries share their private information, $\sigma^{j}\left(p^{A}, p^{B}\right)=\left(p^{A}, p^{B}\right)$. Then the efficient choice $\delta^{E}\left(p^{A}, p^{B}\right)$ is the solution of the following minimization problem

$$
\begin{gathered}
d^{E}=\left(d^{E, A}\left(p^{A}, p^{B}\right), d^{E, B}\left(p^{A}, p^{B}\right)\right) \in \arg \min E[W] \Leftrightarrow \\
\Leftrightarrow d^{E}=\left(d^{E, A}\left(p^{A}, p^{B}\right), d^{E, B}\left(p^{A}, p^{B}\right)\right) \in \\
\in \arg \min E\left[\left\{\gamma d^{A}+\frac{a^{S R}}{p^{A}+p^{B}+p^{A} d^{A}}+\gamma d^{B}+\frac{\left(1-a^{S R}\right)}{p^{A}+p^{B}+p^{B} d^{B}}\right\}\right]
\end{gathered}
$$

where the expectation is taken with respect to the possible states of nature $\left(p^{A}, p^{B}\right) \in\{1,2\} \times\{1,2\}$.

In the Appendix A, we derived the following result 


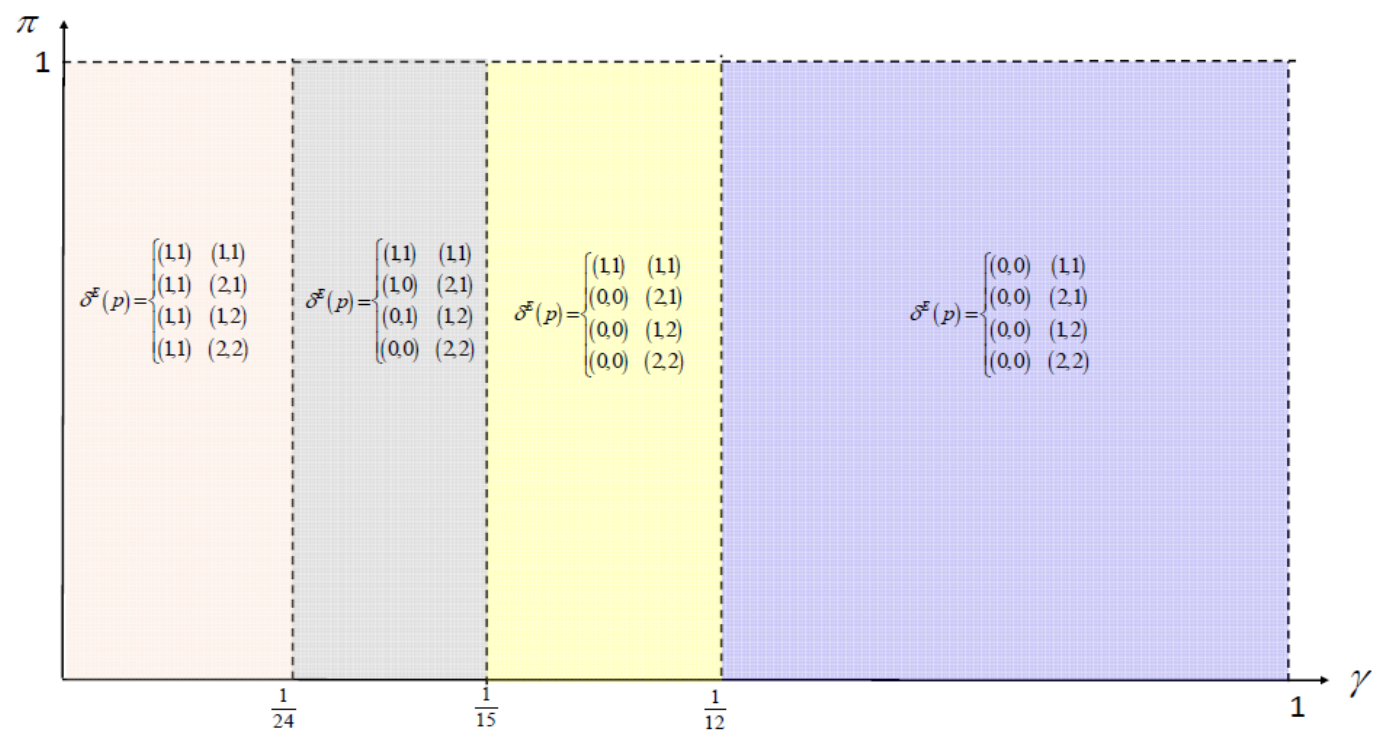

Figure 9: Countries' efficient policies with shared information

Proposition 2 When the countries share their private information, the efficient counter-terrorism defensive policy choices do not depend on the country historical involvement in foreign intervention $(1-\pi)$, but only on voters' propensity towards securization policies $(1-\gamma)$. In particular

1. when voters' propensity towards securization policies is low (high $\gamma$ ), efficient defensive policies are uniformly weak;

2. when voters' propensity towards securization policies is low/intermediate, efficient defensive policies are strong if active counter-terrorism policies are not significant for both countries and weak otherwise;

3. when voters' propensity towards securization policies is high/intermediate, efficient defensive policies are strong if active counterterrorism policies are not significant for both countries or for the country with significant active measures, weak otherwise;

4. when voters' propensity towards securization policies is high, efficient defensive policies are uniformly strong.

Graphically, we get the following picture:

Let us stress that now the probability $\pi$, i.e. the likelihood of non involvement in foreign interventionism, is irrelevant because there is no more private information between the countries. Moreover, as before, efficiency requires that 
stronger defensive policies are associated to stronger voters' request for securization policies (low $\gamma$ ), however for intermediate levels of $\gamma$, a strong defensive policy is associated to unilateral active counter-terrorism policy or to bilateral lack of interventionism.

\section{The Equilibrium Policy Choices}

To solve the game, we consider Perfect Bayesian Equilibria (PBE) in pure strategies as solution concept. Hence, to solve the game we work backward. However, the third stage is not strategic because of our assumption of probabilistic voting. Hence we start from the terrorists' choice, that depends on their beliefs. As usual with signalling games, one of the main problems in characterizing the set of possible PBE is the indeterminacy of the out-of-equilibrium beliefs. A common practice in this class of games, is the use of some form of forward induction to solve such indeterminacy problem, however in this case this criterion is not applicable, because there is no clear indication that a deviation is more likely for a type than for the other. Thus, we prefer to assume passive updating, i.e. the idea that out-of-equilibrium actions do not convey any relevant information that requires to change priors.

\subsection{The Terrorists Sequential Rational Choices}

To derive the PBE, we start with terrorists sequential rational choice as a function of their beliefs. The terrorists' choice depends of the observed

$$
\left(d^{A}, d^{B}\right) \in\{0,1\} \times\{0,1\}
$$

hence it does not directly depend on the institutional scenario. Of course, what might be different are terrorists' beliefs that depend on the governments' policy choice. Formally, the terrorists sequential rational choice is given by the solution to the following maximization problem

$$
\max _{\left\{a^{A}\left(d^{A}, d^{B}\right), a^{B}\left(d^{A}, d^{B}\right) \mid a+a^{B}=1\right\}} E\left(\frac{E\left(p^{A} \mid d^{A}, d^{B}\right) a\left(d^{A}, d^{B}\right)}{p^{A}+p^{B}+p^{A} d^{A}}+\frac{E\left(p^{B} \mid d^{A}, d^{B}\right) a^{B}\left(d^{A}, d^{B}\right)}{p^{A}+p^{B}+p^{B} d^{B}}\right) .
$$

In appendix $\mathrm{B}$, we derive ${ }^{17}$ the terrorists' sequential best replies at each information set as a function of their beliefs, a crucial result to derive the PBE in any institutional scenario.

\subsection{Perfect Bayesian Equilibria in Scenario 1 with Fully Decentralization}

For the governments, there are 2 possible information set and 2 actions for each information set, i.e. 4 possible strategies, hence there are 16 possible

${ }^{17}$ See Lemma 1. 
strategy profiles. In Appendix C, we characterize the entire set of equilibria. In particular, to find a precise characterization of the set of $\mathrm{PBE}$, when $a^{* A}\left(d^{A}, d^{B}\right) \in[0,1]$ we consider the expected value of terrorist sequential rational action, i.e. $a^{*}=\frac{1}{2}$. Then, we get the following result.

Proposition 3 The counter-terrorism game with full decentralization has the following set of pure strategy Perfect Bayesian equilibria that depend on each country historical involvement in foreign intervention $(1-\pi)$ and on voters' propensity towards securization policies $(1-\gamma)$ :

1. When voters' propensity towards securization policies is high (small $\gamma$ ), for any historical involvement in foreign intervention $(1-\pi)$ there is a PBE such that it is sequentially rational to play uninformly strong defensive policies, and terrorists may attack both countries;

2. When voters' propensity towards securization policies is high/intermediate (small/intermediate $\gamma$ ), for any historical involvement in foreign intervention $(1-\pi)$ there is a PBE such that it is sequentially rational to play strong defensive policies when active policies are not significant, and terrorists attack the country when defensive policies are weak;

3. When voters' propensity towards securization policies increases with the probability of historical involvement in foreign intervention, there are two PBE such that it is sequentially rational for a country to play strong defensive policies when active policies are not significant and for the other country to play uniformly weak defensive policies, and terrorists attack the country with weak defensive policies;

4. When voters' propensity towards securization policies is low or intermediate, for any historical involvement in foreign intervention there is a PBE such that it is sequentially rational to uniformly play weak defensive policies, and terrorists may attack both countries.

Let us stress that for many parameters' values there are multiple equilibria, as the following figure shows.

\subsection{Perfect Bayesian Equilibria in Scenario 2 with Intelli- gence Cooperation}

For each government there are 4 possible information set and 2 actions for each information set, i.e. 16 possible strategies, hence there are 256 possible strategy profiles. Hence, it would be unfeasible to consider all these possibilities. However, using the terrorists' sequential rational choice as a function of their possible beliefs as derived in Lemma 1, in Appendix D we are able to characterize the entire set of PBE. 


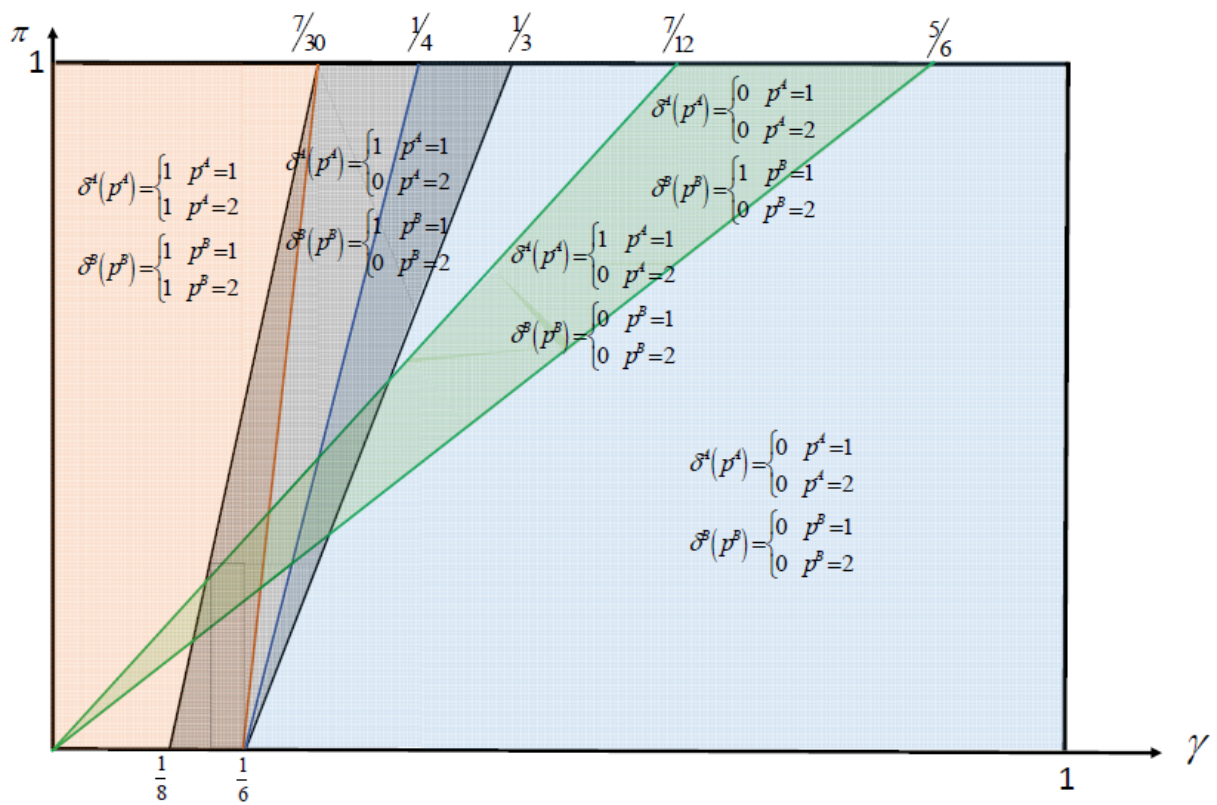

Figure 10: The equilibrium outcomes with full decentralization

Proposition 4 The counter-terrorism game with intelligence cooperation has the following set of pure strategy Perfect Bayesian equilibria that does not depend on each country historical involvement in foreign intervention $(1-\pi)$, but only on voters' propensity towards securization policies $(1-\gamma)$ :

1. When voters' propensity towards securization policies is low (high $\gamma$ ), defensive policies are uniformly weak for both countries, and terrorists may attack both countries;

2. When voters' propensity towards securization policies is low/intermediate, there are multiple equilibria: one equilibrium where defensive policies are uniformly weak for both countries and terrorists may attack both countries, and two equilibria where a country with weak active policy has strong defensive policies and terrorists attack the country with low defensive policy;

3. When voters' propensity towards securization policies is intermediate/low, there are multiple equilibria: one equilibrium where defensive policies are uniformly weak for both countries and terrorists may attack both countries, and two equilibria where a country has strong defensive policies unless both countries active policies are strong, and terrorists attack the country with weak defensive policy;

4. When voters' propensity towards securization policies is intermediate/high there are multiple equilibria: one equilibrium where defensive policies are 


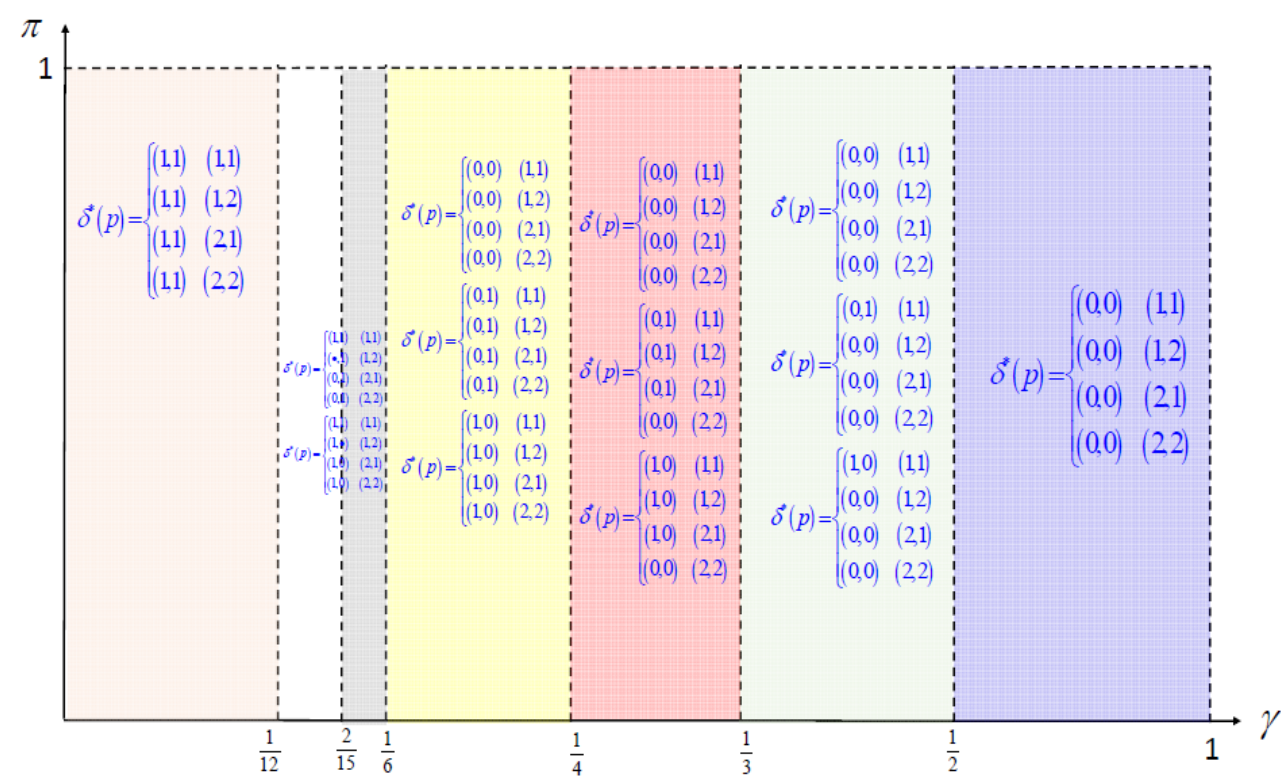

Figure 11: Equilibrium outcomes with intelligence cooperation

uniformly weak for both countries and terrorists may attack both countries, and two equilibria where one of the country has strong defensive policies notwithstanding the active policies and terrorists attack the country with weak defensive policy;

5. When voters' propensity towards securization policies is high/intermediate, there are multiple equilibria where one country defensive policies are uniformly strong and the other country has strong defensive policies only when both countries have weak active policies and terrorists attack the country with sometime weak defensive policies;

6. When voters' propensity towards securization policies is high, there are multiple equilibria where one country defensive policies are uniformly strong and the other country has strong defensive policies only when the other country has weak active policies and terrorists attack the country with sometime weak defensive policies;

7. When voters' propensity towards securization policies is very strong, defensive policies are uniformly strong for both countries, and terrorists may attack both countries.

Graphically, the situation is the following 


\subsection{Perfect Bayesian Equilibria in Scenario 3 with Unan- imous Policy Cooperation}

As for scenario 2, there are 4 possible information set and 4 actions for each information set, hence there are 256 possible strategy profiles. Hence, it would be unfeasible to consider all these possibilities. However, using the terrorists' sequential rational choice as a function of their possible beliefs as derived in Lemma 1, in Appendix E we are able to characterize the entire set of PBE.

Proposition 5 The counter-terrorism game with unanimous policy cooperation has the following set of pure strategy Perfect Bayesian equilibria that does not depend on each country historical involvement in foreign intervention $(1-\pi)$, but only on voters' propensity towards securization policies $(1-\gamma)$ :

1. When voters' propensity towards securization policies is low (high $\gamma$ ), defensive policies are uniformly weak for both countries, and terrorists may attack both countries;

2. When voters' propensity towards securization policies is low/intermediate, defensive policies are strong for both countries when both countries active policies are weak, otherwise defensive policies are weak, and terrorists may attack both countries;

3. When voters' propensity towards securization policies is high/intermediate, defensive policies are weak for both countries only when both countries active policies are strong, otherwise both countries defensive policies are strong, and terrorists may attack both countries;

4. When voters' propensity towards securization policies is high, defensive policies are uniformly strong for both countries, and terrorists may attack both countries.

Graphically, the situation is the following

\subsection{Remarks on the Equilibria in the Full Decentralization Scenario}

The comparison between the PBE and the efficient countries' choices of the full decentralized scenario is illustrated in the following figure.

Remarks 1 Three aspects are particular important:

1. in equilibrium, the countries will never exploit the advantage of having a strong active policy using a strong defensive policy, which is the efficient choice when the citizens evaluate quite significantly a defensive policy and the probability of historical involvement in foreign intervention is strong, i.e. when $\gamma$ and $\pi$ are low intermediate; 


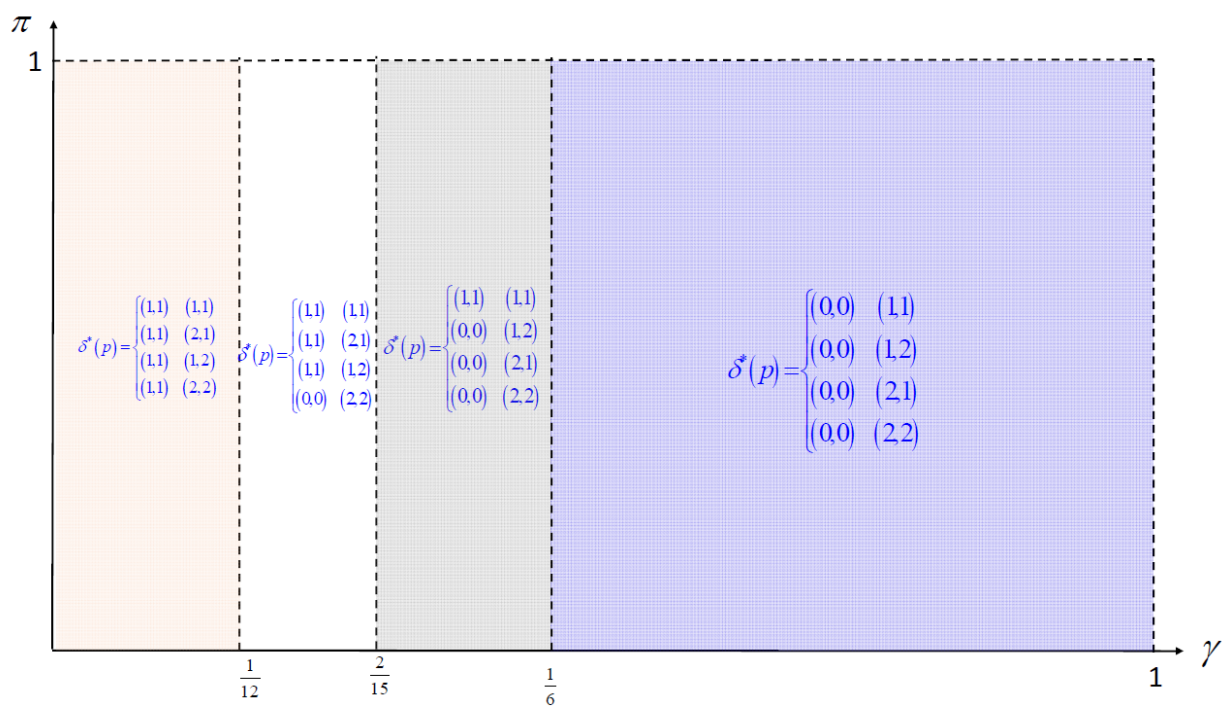

Figure 12: Equilibrium outcomes in a unanimous political union

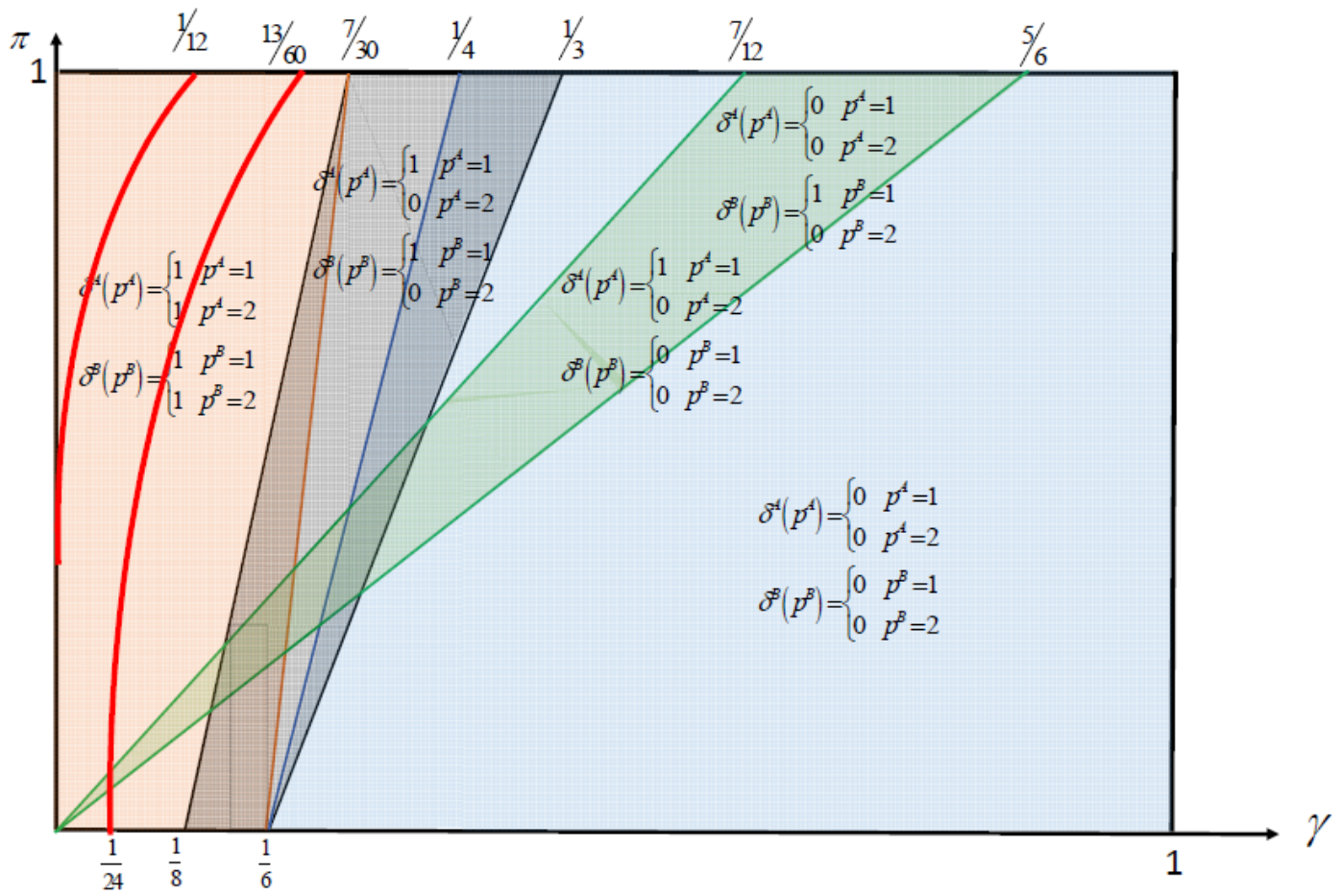

Figure 13: The inefficiency of defensive policies with full decentralization 


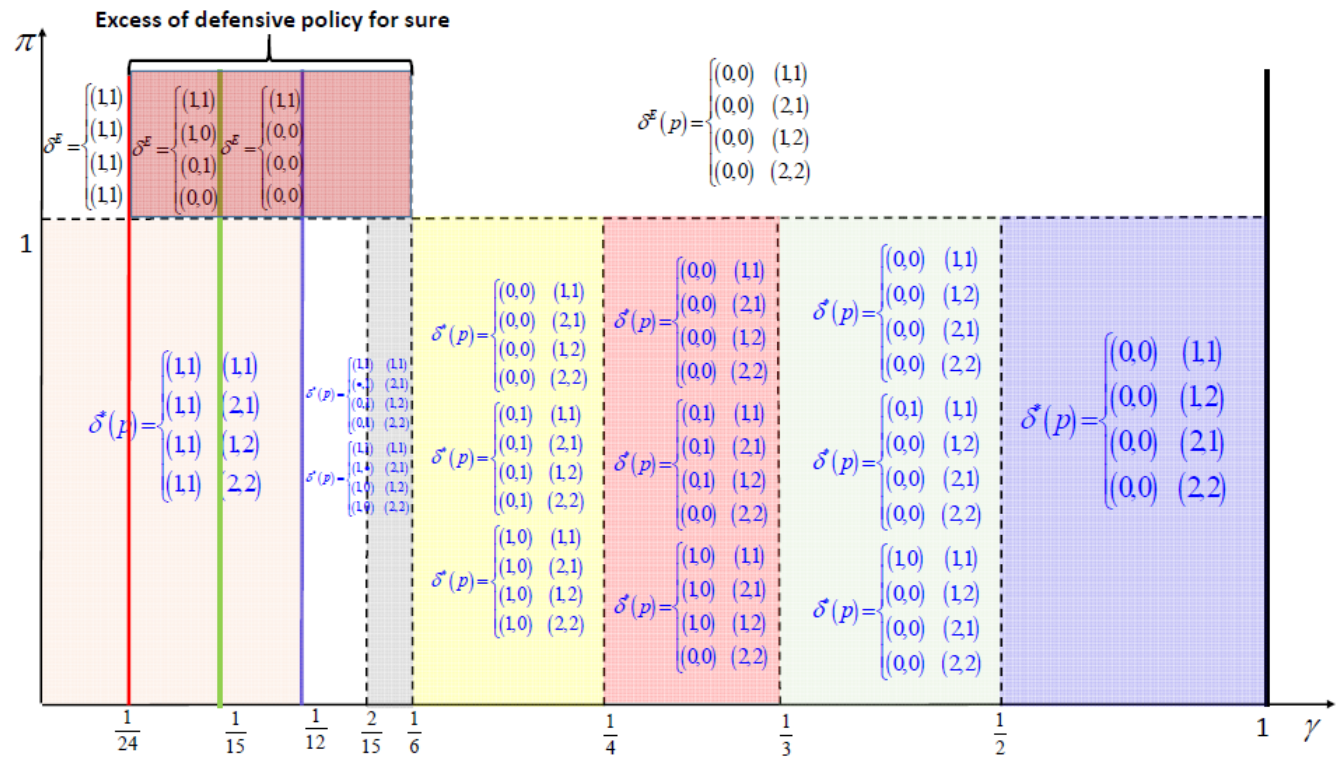

Figure 14: The inefficiency of defensive policies with intelligence cooperation

2. in equilibrium, the countries will invest too much and too often in defensive policies, unless the citizens evaluate strongly a defensive policy and the probability of historical involvement in foreign intervention is weak, i.e. when $\gamma$ is low and $\pi$ is high;

3. in equilibrium the efficient choices of avoiding defensive policies is the unique equilibrium only when the citizens are not very interested in a defensive policy and the probability of historical involvement in foreign intervention is high, i.e. when $\gamma$ is high and $\pi$ is low intermediate; otherwise there are multiple equilibria with an excess spending in defensive policies when there is no active counter-terrorism policy.

\subsection{Remarks on the Equilibria in the Intelligence Coop- eration Scenario}

The comparison between the PBE and the efficient countries' choices when there is intelligence cooperation are illustrated in the following figure.

Remarks 2 Four aspects are particular important

1. As expected, both equilibrium and efficient choices do not depend on the likelihood of having historical involvement in foreign intervention, since there is full sharing of information between countries; 


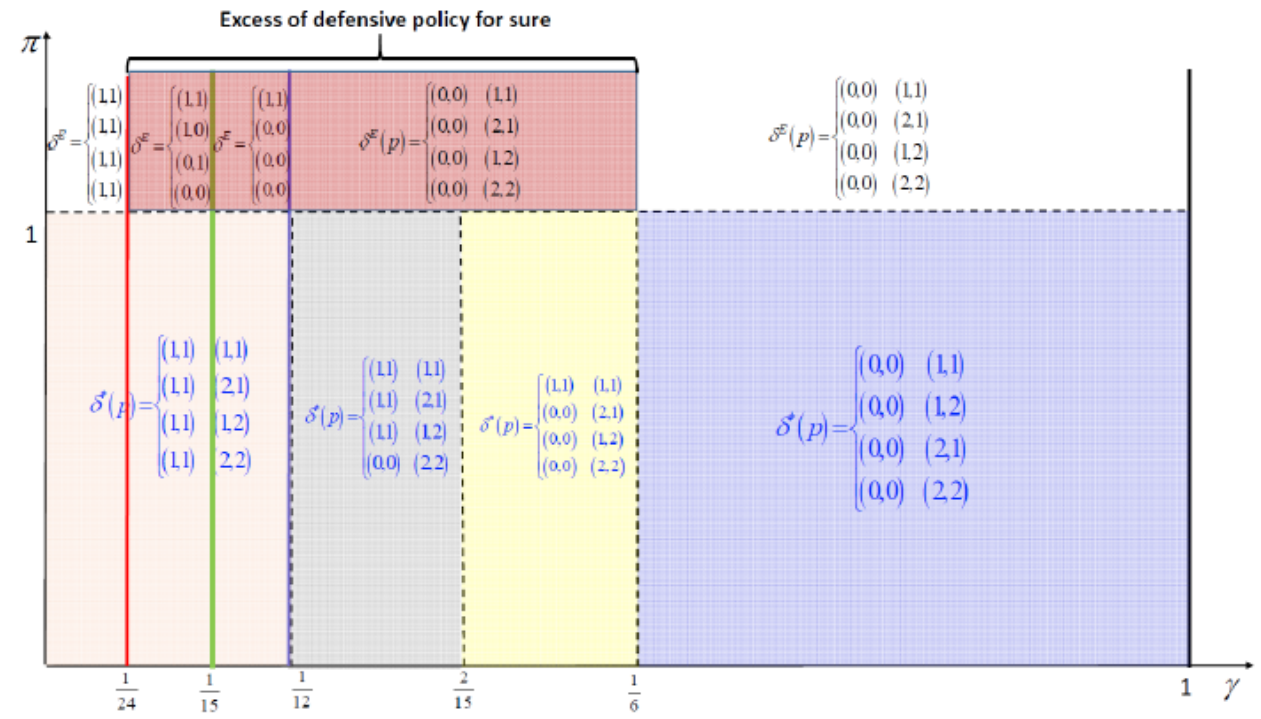

Figure 15: The inefficiency of defensive policies with unanimous political union

2. when the citizens evaluate defensive policies very strongly ( $\gamma$ very low) or when they don't evaluate very much defensive policies ( $\gamma$ high), then the countries' equilibrium choices are efficient;

3. when the citizens evaluate defensive policies strongly ( $\gamma$ low), then in equilibrium there is surely an excess of defensive policies

4. when the citizens' evaluation of defensive policies is intermediate ( $\gamma$ intermediate), there is one efficient equilibrium, however there are also inefficient equilibria because of excessive defensive policies.

Summing up, the coordination of intelligence reduces the likelihood of inefficient policies, however the possibility of inefficient excessive defensive policies persists, in particular when they are significantly evaluated by the citizens.

\subsection{Remarks on the Equilibria in the Unanimous Political Union Scenario}

The comparison between the PBE and the efficient countries' choices in the Political Union scenario are illustrated in the following figure.

Remarks 3 Four aspects are particular important 
1. As expected, both equilibrium and efficient choices do not depend on the likelihood of having historical involvement in foreign intervention, since between countries there is full sharing of information;

2. when the citizens evaluate defensive policies very significantly ( $\gamma$ very low) or when they don't evaluate very much defensive policies ( $\gamma$ high), then the countries' equilibrium choices are surely efficient, as in the scenario of intelligence cooperation;

3. when the citizens' evaluation of defensive policies is intermediate ( $\gamma$ intermediate), then the equilibria are inefficient because of excessive defensive policies;

4. most importantly, the unanimous political union is less likely to be efficient that the case of coordination of intelligence.

\section{Conclusion}

Our paper differs from the previous literature in several important aspects. It emphasizes the role of past history in determining the involvement in active foreign antiterrorism, the backlash effects of such involvement, the importance of democratic accountability in the choice of defensive policies and, crucially, the relevance of the institutional scenarios to fix the possibility of policy cooperation between countries and thus in inducing more or less efficient policies. Two aspects play a crucial role in the study of the (in)efficient defensive policy choices, the citizens' propensity towards securization policies and the institutional scenario. We believe that the analysis of the effects of the citizens' propensity towards securization policies within the four institutional scenarios is quite informative, even if the model is simple. In particular, we show that while the best institutional setting to eliminate the consequences of negative externalities is clearly a full political union and the worst case is the situation of full decentralization, from a mere efficiency point of view, political cooperation with unanimity is worse than a simple intelligence coordination. This is partially surprising, however it remind us of the second best principle and of the well known fact that we cannot guarantee efficient outcomes in coordination games with perfectly coinciding interests ${ }^{18}$ In this case, the situation is even more complex because the countries' interests are not perfectly aligned and any decision should be taken unanimously. A further interesting feature of this model is that inefficiency is usually more likely for intermediate values of voters' propensity towards securization policies. This means that the institutional scenario is less crucial when voters are uniformously in favor or against securization policies, while it matters mostly with voters' fragmentation or in intermediate situations.

An obvious limitation of the analysis is the explicit functional form for players' payoffs, however this approach is quite common in the literature ${ }^{19}$ and al-

\footnotetext{
${ }^{18}$ See e.g. Cooper 2010.

${ }^{19}$ See e.g. Alesina and Spolaore 1997 and 2006, Alesina et al. 2000 and 2005.
} 
lows the explicit characterization of the set of the game's equilibria. Besides this methodological choice, many important aspects are missing from our analysis. First of all, we think it is crucial to develop the analysis of alternative institutional scenarios with a more detailed model of political cooperation. A second important aspect is to consider the explicit choice of alternative active counterterrorism policies. Finally, our models abstract by assuming just two targeted nations. We think it will be useful to generalize the model to $n$ countries to study the effects of changes in the number of member states on efficiency. More generally, we believe that the integration between studies of security policies with works on political unions is extremely promising. 


\section{References}

[1] Alesina, A., Spolaore, E. (1997). On the Number and Size of Nations. The Quarterly Journal of Economics, 112(4), 1027-1056.

[2] Alesina, A., Angeloni, I., \& Etro, F. (2005). International Unions. The American Economic Review, 95(3), 602-615.

[3] Alesina, A., Spolaore, E. , Wacziarg, R. (2000) Economic Integration and Political Disintegration, American Economic Review, 90 (5), 1276-97.

[4] Alesina, A., Spolaore, E. (2006). Conflict, defense spending, and the number of nations. European Economic Review 50(1): 91-120.

[5] Amir, R., F. Garcia, and M. Knauff (2010), Symmetry-breaking in twoplayer games via strategic substitutes and diagonal nonconcavity: A synthesis, Journal of Economic Theory vol. 145, N.5, pp. 1968-1986.

[6] Arce D. G. M. and T. Sandler (2005), Counterterrorism: A Game-Theoretic Analysis, The Journal of Conflict Resolution, Vol. 49, No. 2, pp. 183-200.

[7] Arce, M., Sandler, T., (2007), Terrorist signaling and the value of intelligence. Brit. J. Polit. Sci. 37, 576-586.

[8] Arce, D., \& Sandler, T. (2010). Terrorist Spectaculars: Backlash Attacks and the Focus of Intelligence. The Journal of Conflict Resolution, 54(2), 354-373.

[9] Arce D. G. and K. Siqueira (2014), Motivating operatives for suicide missions and conventional terrorist attacks, Journal of Theoretical Politics, Volume 26, issue: 4, pp. 677-695.

[10] Bandyopadhyay S. and Todd Sandler (2011), The Interplay Between Preemptive and Defensive Counterterrorism Measures: A Two-stage Game, Economica, Vol. 78, No. 311, pp. 546-564

[11] Bandyopadhyay S. and T. Sandler (2014), Immigration policy and counterterrorism, Journal of Public Economics 110, pp. 112-123.

[12] Brueck T., D. Meierrieks and F. Schneider (2008), "The Economics of Terrorism and Counter-Terrorism: A Survey (Part I), CESifo Working Paper N. 3011.

[13] Brueck T., D. Meierrieks and F. Schneider (2008), "The Economics of Terrorism and Counter-Terrorism: A Survey (Part II), CESifo Working Paper N. 3012.

[14] Brueck T. and D. Meierrieks (2015), "The Economics of Counterterrorism: A Survey, Journal of Economic Surveys, Vol. 29, No. 1, pp. 131-157 
[15] Bueno de Mesquita, Ethan (2007). "Politics and the Suboptimal Provision of Counterterror," International Organization 61.

[16] Bueno De Mesquita E. and E. S. Dickson (2007), The Propaganda of the Deed: Terrorism, Counterterrorism and Mobilization, American Journal of Political Science, vol. 51, no. 2, pp. 364-381.

[17] Cavalli, F., M. Gilli, and A. Naimzada (2017), A new approach to contest models, Tech. rep. 364, University of Milan Bicocca, Department of Economics, Management and Statistics, Working Paper, SSRN Series.

[18] Cárceles-Poveda E. and Y. Taumana (2011), A strategic analysis of the war against transnational terrorism, Games and Economic Behavior 71, pp. 49-65.

[19] Cooper R. (2010), Coordination Games, Cambridge, Cambridge University Press.

[20] Das S. P. and S. Lahiri (2006), A Strategic Analysis of Terrorist Activity and Counter-Terrorism Policies, The B.E. Journal of Theoretical Economics, vol. 6 , no. 1, pp. 1-28.

[21] de Oliveira, A. R., Faria, J. R., \& Silva, E. C. D. (2018). Transnational Terrorism: Externalities and Coalition Formation. Journal of Conflict Resolution, 62(3), 496-528.

[22] Enders, W. and T. Sandler (1993) 'Effectiveness of anti-terrorism policies: vector- autoregression-intervention analysis,' American Political Science Review 87, 829-44.

[23] Enders, W. and T. Sandler (2006a) 'Distribution of transnational terrorism among countries by income classes and geography after $9 / 11$,' International Studies Quarterly 50, 367-93

[24] Enders W. and T.Sandler (2006b), The Political Economy of Terrorism, Cambridge University Press, Cambridge.

[25] Enders, W., T. Sandler, and J. Cauley. 1990. UN Conventions, Technology, and Retaliation in the Fight against Terrorism: An Econometric Evaluation. Terrorism and Political Violence 2:83-105.

[26] English Robert (2016), Does Terrorism work? Oxford University Press, Oxford.

[27] Europol (2009-2018) EU TERRORISM SITUATION AND TREND REPORT https://www.europol.europa.eu/activities-services/mainreports/eu-terrorism-situation-and-trend-report

[28] Feddersen, T., \& Pesendorfer, W. (1998). Convicting the Innocent: The Inferiority of Unanimous Jury Verdicts under Strategic Voting. American Political Science Review, 92(1), 23-35. doi:10.2307/2585926 
[29] Gaibulloev K. and T. Sandler (2019), What We Have Learned about Terrorism since 9/11, Journal of Economic Literature 57(2), 275-328

[30] Gentzkow, M. and E. Kamenica 2011. Bayesian persuasion, American Economic Review, 101(6).

[31] Gottlieb Stuart (ed) (2014), Debating Terrorism and Counterterrorism, SAGE, London.

[32] Granoty, D, M. Maschleryy and J. Shalev, (2003) Voting for voters: The unanimity case, International Journal of Game Theory, 31(2):155-202

[33] Harstad, B. (2010), Strategic delegation and voting rules, Journal of Public Economics 94, 102-113.

[34] Kalai E. and D. Samet 1985, Unanimity Games and Pareto Optimality, International Journal of Game Theory, Vol. 14, Issue 1, page 41-50.

[35] Kunreuther, H. and Heal, G. (2003). Interdependent security, Journal of Risk and Uncertainty, 26, 231-249.

[36] Lapan, H.E., Sandler, T., (1993). Terrorism and signalling. Europ. J. Polit. Economy 9 (3), 383-398.

[37] Levy, G. (2007). Decision Making in Committees: Transparency, Reputation, and Voting Rules. The American Economic Review, 97(1), 150-168.

[38] Li Q. (2005), Does Democracy Promote or Reduce Transnational Terrorist Incidents?, The Journal of Conflict Resolution, Vol. 49, No. 2, pp. 278-297

[39] Nesser, P. (2018). Europe hasn't won the war on terror", https://www.politico.eu/article/europe-hasnt-won-the-war-on-terror/.

[40] Overgaard P. B., (1994). The Scale of terrorist attacks as a signal of resources. J. Conflict Resolution 38 (3), 452-478.

[41] Rogna M. (2019), Coalition Formation and Bargaining Protocols: A Review of the Literature, Journal of Economic Surveys Vol. 33, No. 1, pp. 226-251

[42] Rosendorff, B. Peter, and Todd Sandler (2004), Too much of a good thing? The active response dilemma, Journal of Conflict Resolution 48, 657-71.

[43] Sandler, T. (2003), Collective action and transnational terrorism. World Economy 26 (6): 779-802.

[44] Sandler T. and W. Enders (2004), An Economic Perspective on Transnational Terrorism, European Journal of Political Economy, 20, 301-316.

[45] Sandler T. and K. Siqueira (2006), Global Terrorism: Deterrence versus Pre-Emption, The Canadian Journal of Economics, Vol. 39, No.4, pp. 13701387 
[46] Siqueira K. (2003), Participation in organized and unorganized protests and rebellions, European Journal of Political Economy, vol. 19, no. 4, pp. 651-918.

[47] Siqueira K. and T. Sandler (2006), Terrorist versus the Government: Strategic Interaction, Support, and Sponsorship, The Journal of Conflict Resolution, vol. 46, no.1, pp. 878-898.

[48] Tullock, G. (1980), Efficient rent seeking. In: Buchanan, J., Tollison, R., Tullock, G. (Eds.), Toward a Theory of the Rent Seeking Society. Texas A\&M University Press, pp. 97-112.

[49] Tsebelis, G. (2002), Veto Players: How Political Institutions. Princeton, Princeton UP and Russell Sage Foundation. 


\section{Part I}

\section{Appendix}

\section{Appendix A: Calculation of Terrorists' Sequen- tial Rational Choices}

To simplify notation, write $a$ instead of $a^{A}$. The terrorists' rational choice in the second stage does not depend on the first stage structure, only on the observed $\left(d^{A}, d^{B}\right) \in\{0,1\} \times\{0,1\}$

$$
\max _{a\left(d^{A}, d^{B}\right) \in[0,1]} E\left(\frac{p^{A} a\left(d^{A}, d^{B}\right)}{p^{A}+p^{B}+p^{A} d^{A}}+\frac{p^{B}\left[1-a\left(d^{A}, d^{B}\right)\right]}{p^{A}+p^{B}+p^{B} d^{B}} \mid d^{A}, d^{B}\right)=
$$

where the expectation depend on $T$ beliefs on $\left(p^{A}, p^{B}\right)$ given the observation $\left(d^{A}, d^{B}\right)$.To simplify let we use the following notation:

$$
\begin{aligned}
& \mu^{11 \mid 00}=\mu\left\{\left(p^{A}, p^{B}\right)=(1,1) \mid\left(d^{A}=0, d^{B}=0\right)\right\} \\
& \mu^{21 \mid 00}=\mu\left\{\left(p^{A}, p^{B}\right)=(2,1) \mid\left(d^{A}=0, d^{B}=0\right)\right\} \\
& \mu^{12 \mid 00}=\mu\left\{\left(p^{A}, p^{B}\right)=(1,2) \mid\left(d^{A}=0, d^{B}=0\right)\right\} \\
& \mu^{22 \mid 00}=\mu\left\{\left(p^{A}, p^{B}\right)=(2,2) \mid\left(d^{A}=0, d^{B}=0\right)\right\} \\
& \mu^{11 \mid 10}=\mu\left\{\left(p^{A}, p^{B}\right)=(1,1) \mid\left(d^{A}=1, d^{B}=0\right)\right\} \\
& \mu^{21 \mid 10}=\mu\left\{\left(p^{A}, p^{B}\right)=(2,1) \mid\left(d^{A}=1, d^{B}=0\right)\right\} \\
& \mu^{12 \mid 10}=\mu\left\{\left(p^{A}, p^{B}\right)=(1,2) \mid\left(d^{A}=1, d^{B}=0\right)\right\} \\
& \mu^{22 \mid 10}=\mu\left\{\left(p^{A}, p^{B}\right)=(2,2) \mid\left(d^{A}=1, d^{B}=0\right)\right\} \\
& \mu^{11 \mid 01}=\mu\left\{\left(p^{A}, p^{B}\right)=(1,1) \mid\left(d^{A}=0, d^{B}=1\right)\right\} \\
& \mu^{21 \mid 01}=\mu\left\{\left(p^{A}, p^{B}\right)=(2,1) \mid\left(d^{A}=0, d^{B}=1\right)\right\} \\
& \mu^{12 \mid 01}=\mu\left\{\left(p^{A}, p^{B}\right)=(1,2) \mid\left(d^{A}=0, d^{B}=1\right)\right\} \\
& \mu^{22 \mid 01}=\mu\left\{\left(p^{A}, p^{B}\right)=(2,2) \mid\left(d^{A}=0, d^{B}=01\right)\right\} \\
& \mu^{11 \mid 11}=\mu\left\{\left(p^{A}, p^{B}\right)=(1,1) \mid\left(d^{A}=1, d^{B}=1\right)\right\} \\
& \mu^{21 \mid 11}=\mu\left\{\left(p^{A}, p^{B}\right)=(2,1) \mid\left(d^{A}=1, d^{B}=1\right)\right\} \\
& \mu^{12 \mid 11}=\mu\left\{\left(p^{A}, p^{B}\right)=(1,2) \mid\left(d^{A}=1, d^{B}=1\right)\right\} \\
& \mu^{22 \mid 11}=\mu\left\{\left(p^{A}, p^{B}\right)=(2,2) \mid\left(d^{A}=1, d^{B}=1\right)\right\}
\end{aligned}
$$

Then 
1. When $\left(d^{A}, d^{B}\right)=(0,0)$

$$
\begin{gathered}
E\left(\frac{p^{A} a}{p^{A}+p^{B}+p^{A} d^{A}}\right)=\frac{a\left(3+\mu^{2,1 \mid 0,0}-\mu^{1,2 \mid 0,0}\right)}{6} \\
E\left(\frac{p^{B}\left(1-a^{A}\right)}{p^{A}+p^{B}+\left(p^{B}+1\right) d^{B}+1}\right)=\frac{(1-a)}{2}-\frac{(1-a) \mu^{2,1 \mid 0,0}}{6}+\frac{(1-a) \mu^{1,2 \mid 0,0}}{6}
\end{gathered}
$$

thus

$$
a^{S R}(0,0) \in\left\{\begin{array}{cc}
\{1\} & \mu^{2,1 \mid 0,0} \geq \mu^{1,2 \mid 0,0} \\
{[0,1]} & \mu^{2,1 \mid 0,0}=\mu^{1,2 \mid 0,0} \\
\{0\} & \mu^{2,1 \mid 0,0} \leq \mu^{1,2 \mid 0,0}
\end{array}\right.
$$

2. When $\left(d^{A}, d^{B}\right)=(1,0)$

$$
\begin{aligned}
E\left(\frac{p^{A} a}{p^{A}+p^{B}+p^{A} d^{A}}\right) & =\frac{a}{3}+\frac{a\left(4 \mu^{2,1 \mid 1,0}-5 \mu^{1,2 \mid 1,0}\right)}{60} \\
E\left(\frac{p^{B}\left(1-a^{A}\right)}{p^{A}+p^{B}+\left(p^{B}+1\right) d^{B}+1}\right) & =\frac{(1-a)}{2}-\frac{(1-a) \mu^{2,1 \mid 1,0}}{6}+\frac{(1-a) \mu^{1,2 \mid 1,0}}{6}
\end{aligned}
$$

thus

$$
a^{S R}(1,0) \in\left\{\begin{array}{cl}
\{1\} & \mu^{2,1 \mid 1,0} \geq \frac{15 \mu^{1,2 \mid 1,0}+10}{14} \\
{[0,1]} & \mu^{2,1 \mid 1,0}=\frac{15 \mu^{1,2 \mid 1,0}+10}{14} \\
\{0\} & \mu^{2,1 \mid 1,0} \leq \frac{15 \mu^{1,2 \mid 1,0}+10}{14}
\end{array}\right.
$$

3. When $\left(d^{A}, d^{B}\right)=(0,1)$

$$
\begin{gathered}
E\left(\frac{p^{A} a}{p^{A}+p^{B}+p^{A} d^{A}}\right)=\frac{a}{2}+\frac{a \mu^{2,1 \mid 0,0}}{6}-\frac{a \mu^{1,2 \mid 0,1}}{6} \\
E\left(\frac{p^{B}\left(1-a^{A}\right)}{p^{A}+p^{B}+\left(p^{B}+1\right) d^{B}+1}\right)=\frac{(1-a)}{3}-\frac{(1-a) \mu^{2,1 \mid 0,1}}{12}+\frac{(1-a) \mu^{1,2 \mid 0,1}}{15}
\end{gathered}
$$

thus

$$
a^{S R}(0,1) \in\left\{\begin{array}{cl}
\{1\} & \mu^{2,1 \mid 0,1} \geq \frac{14 \mu^{1,2 \mid 0,1}-10}{1 \mu^{1,25,1}} \\
{[0,1]} & \mu^{2,1 \mid 0,1}=\frac{14 \mu^{15}-10}{15} \\
\{0\} & \mu^{2,1 \mid 0,1} \leq \frac{14 \mu^{1,2 \mid 0,1}-10}{15}
\end{array}\right.
$$

4. when $\left(d^{A}, d^{B}\right)=(1,1)$

$$
\begin{gathered}
E\left(\frac{p^{A} a}{p^{A}+p^{B}+p^{A} d^{A}}\right)=\frac{a}{3}+\frac{a \mu^{2,1 \mid 1,1}}{15}-\frac{a \mu^{1,2 \mid 1,1}}{12} \\
E\left(\frac{p^{B}\left(1-a^{A}\right)}{p^{A}+p^{B}+\left(p^{B}+1\right) d^{B}+1}\right)=\frac{(1-a)}{3}-\frac{(1-a) \mu^{2,1 \mid 1,1}}{12}+\frac{(1-a) \mu^{1,2 \mid 1,1}}{15}
\end{gathered}
$$


thus

$$
a^{S R}(1,1) \in\left\{\begin{array}{cc}
\{1\} & \mu^{2,1 \mid 1,1} \geq \mu^{1,2 \mid 1,1} \\
{[0,1]} & \mu^{2,1 \mid 1,1}=\mu^{1,2 \mid 1,1} \\
\{0\} & \mu^{2,1 \mid 1,1} \leq \mu^{1,2 \mid 1,1}
\end{array}\right.
$$

So, the terrorists sequential rational $a^{S R}\left(d^{A}, d^{B}\right)$ choices depend on their beliefs as follows:

Lemma 1 The terrorists' sequential rational choices depend on their beliefs as follows:

If $\left\{\begin{array}{c}\mu^{2,1 \mid 0,0} \geq \mu^{1,2 \mid 0,0} \\ \mu^{2,1 \mid 1,0} \geq \frac{15 \mu^{1,2 \mid 1,0}+10}{14} \\ \mu^{2,1 \mid 0,1} \geq \frac{14 \mu^{1,2 \mid 0,1}-10}{15} \\ \mu^{2,1 \mid 1,1} \geq \mu^{1,2 \mid 1,1}\end{array} \quad\right.$ then $\quad a^{S R}\left(d^{A}, d^{B}\right) \in\left\{\begin{array}{l}\{1\} \\ \{1\} \quad\left(d^{A}, d^{B}\right)=(0,0) \\ \{1\} \quad\left(d^{A}, d^{B}\right)=(1,0) \\ \{1\} \quad\left(d^{A}, d^{B}\right)=(0,1)\end{array}\right.$

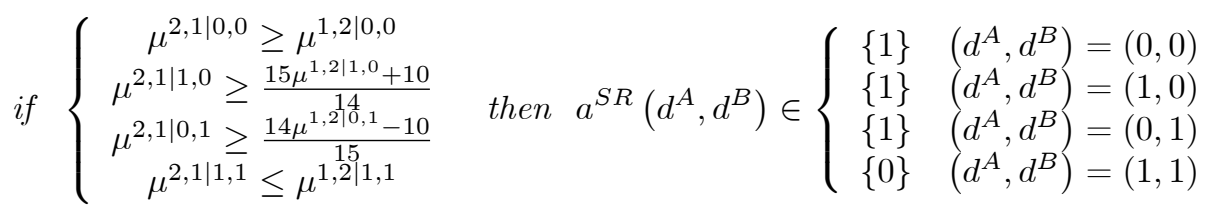

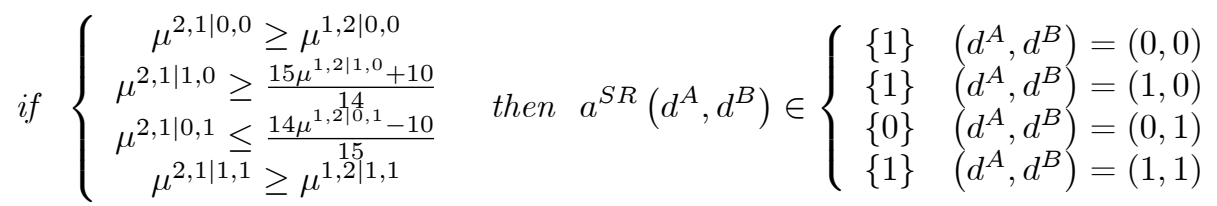

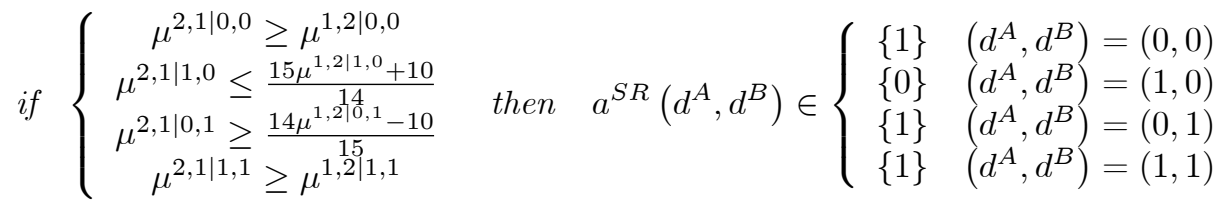

if $\left\{\begin{array}{c}\mu^{2,1 \mid 0,0} \leq \mu^{1,2 \mid 0,0} \\ \mu^{2,1 \mid 1,0} \geq \frac{15 \mu^{1,2 \mid 1,0}+10}{14} \\ \mu^{2,1 \mid 0,1} \geq \frac{14 \mu^{1,2 \mid 0,1}-10}{15} \\ \mu^{2,1 \mid 1,1} \geq \mu^{1,2 \mid 1,1}\end{array} \quad\right.$ then $\quad a^{S R}\left(d^{A}, d^{B}\right) \in\left\{\begin{array}{l}\{0\} \quad\left(d^{A}, d^{B}\right)=(0,0) \\ \{1\} \quad\left(d^{A}, d^{B}\right)=(1,0) \\ \{1\} \quad\left(d^{A}, d^{B}\right)=(0,1) \\ \{1\} \quad\left(d^{A}, d^{B}\right)=(1,1)\end{array}\right.$

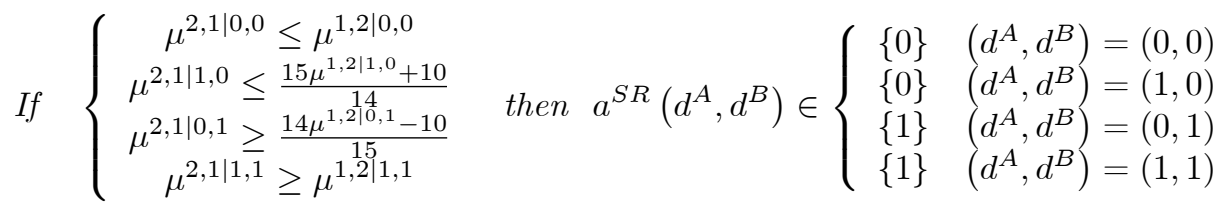

If $\left\{\begin{array}{c}\mu^{2,1 \mid 0,0} \leq \mu^{1,2 \mid 0,0} \\ \mu^{2,1 \mid 1,0} \geq \frac{15 \mu^{1,2 \mid 1,0}+10}{14} \\ \mu^{2,1 \mid 0,1} \leq \frac{14 \mu^{1,2 \mid 0,1}-10}{15} \\ \mu^{2,1 \mid 1,1} \geq \mu^{1,2 \mid 1,1}\end{array} \quad\right.$ then $a^{S R}\left(d^{A}, d^{B}\right) \in \begin{cases}\{0\} & \left(d^{A}, d^{B}\right)=(0,0) \\ \{1\} & \left(d^{A}, d^{B}\right)=(1,0) \\ \{0\} & \left(d^{A}, d^{B}\right)=(0,1) \\ \{1\} & \left(d^{A}, d^{B}\right)=(1,1)\end{cases}$ 


$$
\begin{aligned}
& \text { If }\left\{\begin{array} { c } 
{ \mu ^ { 2 , 1 | 0 , 0 } \leq \mu ^ { 1 , 2 | 0 , 0 } } \\
{ \mu ^ { 2 , 1 | 1 , 0 } \geq \frac { 1 5 \mu ^ { 1 , 2 | 1 , 0 } + 1 0 } { 1 4 } } \\
{ \mu ^ { 2 , 1 | 0 , 1 } \geq \frac { 1 4 \mu ^ { 1 , 2 | 0 , 1 } - 1 0 } { 1 5 } } \\
{ \mu ^ { 2 , 1 | 1 , 1 } \leq \mu ^ { 1 , 2 | 1 , 1 } }
\end{array} \quad \text { then } \quad a ^ { S R } ( d ^ { A } , d ^ { B } ) \in \left\{\begin{array}{l}
\{0\} \\
\{1\} \quad\left(d^{A}, d^{B}\right)=(0,0) \\
\{1\} \quad\left(d^{A}, d^{B}\right)=(1,0) \\
\{0\} \quad\left(d^{A}, d^{B}\right)=(0,1)
\end{array}\right.\right. \\
& \text { If }\left\{\begin{array} { c } 
{ \mu ^ { 2 , 1 | 0 , 0 } \geq \mu ^ { 1 , 2 | 0 , 0 } } \\
{ \mu ^ { 2 , 1 | 1 , 0 } \leq \frac { 1 5 \mu ^ { 1 , 2 | 1 , 0 } + 1 0 } { 1 4 } } \\
{ \mu ^ { 2 , 1 | 0 , 1 } \leq \frac { 1 4 \mu ^ { 1 , 2 | 0 , 1 } - 1 0 } { 1 5 } } \\
{ \mu ^ { 2 , 1 | 1 , 1 } \geq \mu ^ { 1 , 2 | 1 , 1 } }
\end{array} \quad \text { then } \quad a ^ { S R } ( d ^ { A } , d ^ { B } ) \in \left\{\begin{array}{l}
\{1\} \quad\left(d^{A}, d^{B}\right)=(0,0) \\
\{0\} \quad\left(d^{A}, d^{B}\right)=(1,0) \\
\{0\} \quad\left(d^{A}, d^{B}\right)=(0,1) \\
\{1\} \quad\left(d^{A}, d^{B}\right)=(1,1)
\end{array}\right.\right.
\end{aligned}
$$

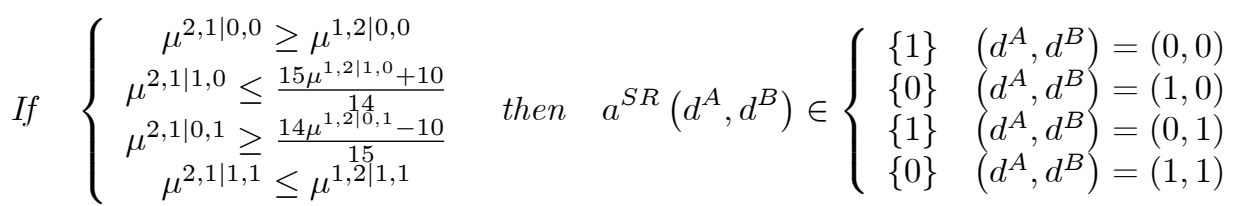

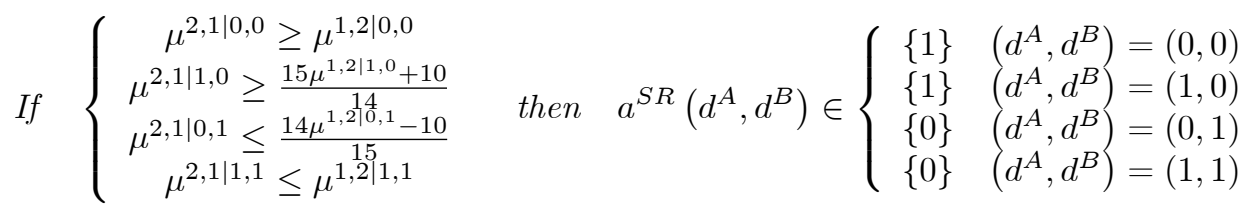

$$
\begin{aligned}
& \text { If }\left\{\begin{array} { c } 
{ \mu ^ { 2 , 1 | 0 , 0 } \leq \mu ^ { 1 , 2 | 0 , 0 } } \\
{ \mu ^ { 2 , 1 | 1 , 0 } \leq \frac { 1 5 \mu ^ { 1 , 2 | 1 , 0 } + 1 0 } { 1 4 } } \\
{ \mu ^ { 2 , 1 | 0 , 1 } \leq \frac { 1 4 \mu ^ { 1 , 2 | 0 , 1 } - 1 0 } { 1 5 } } \\
{ \mu ^ { 2 , 1 | 1 , 1 } \geq \mu ^ { 1 , 2 | 1 , 1 } }
\end{array} \quad \text { then } a ^ { S R } ( d ^ { A } , d ^ { B } ) \in \left\{\begin{array}{l}
\{0\} \\
\{0\} \quad\left(d^{A}, d^{B}\right)=(0,0) \\
\{0\} \quad\left(d^{A}, d^{B}\right)=(1,0) \\
\{1\} \quad\left(d^{A}, d^{B}\right)=(0,1)
\end{array}\right.\right.
\end{aligned}
$$

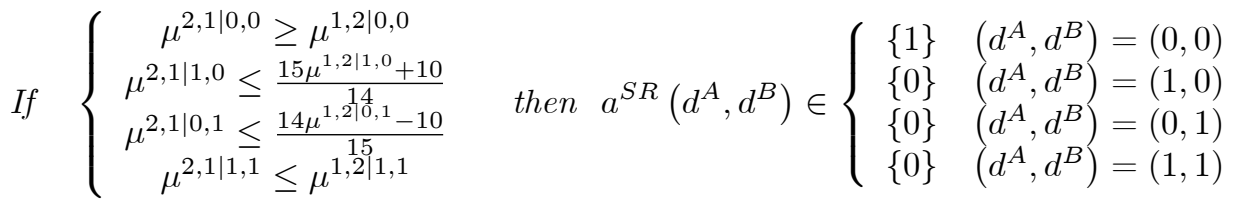

$$
\begin{aligned}
& \text { If }\left\{\begin{array} { c } 
{ \mu ^ { 2 , 1 | 0 , 0 } \leq \mu ^ { 1 , 2 | 0 , 0 } } \\
{ \mu ^ { 2 , 1 | 1 , 0 } \geq \frac { 1 5 \mu ^ { 1 , 2 | 1 , 0 } + 1 0 } { 1 4 } } \\
{ \mu ^ { 2 , 1 | 0 , 1 } \leq \frac { 1 4 \mu ^ { 1 , 2 | 0 , 1 } - 1 0 } { 1 5 } } \\
{ \mu ^ { 2 , 1 | 1 , 1 } \leq \mu ^ { 1 , 2 | 1 , 1 } }
\end{array} \quad \text { then } \quad a ^ { S R } ( d ^ { A } , d ^ { B } ) \in \left\{\begin{array}{c}
\{0\} \quad\left(d^{A}, d^{B}\right)=(0,0) \\
\{1\} \quad\left(d^{A}, d^{B}\right)=(1,0) \\
\{0\} \quad\left(d^{A}, d^{B}\right)=(0,1) \\
\{0\} \quad\left(d^{A}, d^{B}\right)=(1,1)
\end{array}\right.\right. \\
& \text { If }\left\{\begin{array} { c } 
{ \mu ^ { 2 , 1 | 0 , 0 } \leq \mu ^ { 1 , 2 | 0 , 0 } } \\
{ \mu ^ { 2 , 1 | 1 , 0 } \leq \frac { 1 5 \mu ^ { 1 , 2 | 1 , 0 } + 1 0 } { 1 4 } } \\
{ \mu ^ { 2 , 1 | 0 , 1 } \geq \frac { 1 4 \mu ^ { 1 , 2 | 0 , 1 } - 1 0 } { 1 5 } } \\
{ \mu ^ { 2 , 1 | 1 , 1 } \leq \mu ^ { 1 , 2 | 1 , 1 } }
\end{array} \quad \text { then } \quad a ^ { S R } ( d ^ { A } , d ^ { B } ) \in \left\{\begin{array}{l}
\{0\} \quad\left(d^{A}, d^{B}\right)=(0,0) \\
\{0\} \quad\left(d^{A}, d^{B}\right)=(1,0) \\
\{1\} \quad\left(d^{A}, d^{B}\right)=(0,1) \\
\{0\} \quad\left(d^{A}, d^{B}\right)=(1,1)
\end{array}\right.\right.
\end{aligned}
$$

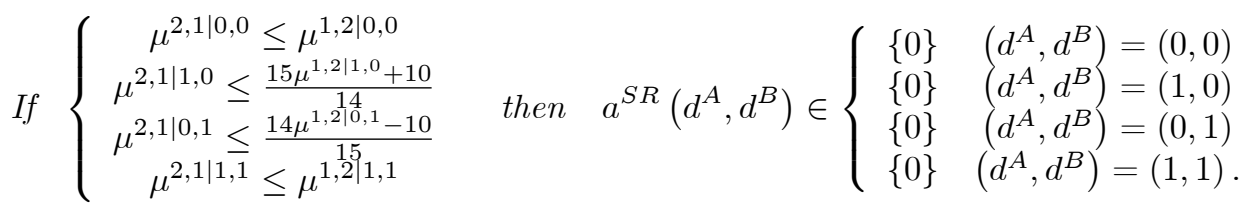




\section{Appendix B: Efficient Choices and Countries' Information}

\subsection{The Countries Efficient Choices with Private Informa- tion}

When there is full decentralization, $\sigma^{j}\left(p^{j}, p^{-j}\right)=p^{j}$. The efficient outcome for the government and the citizens of country $j$ should consider this information limitation, hence it is given by the choice $\delta^{E, j}\left(p^{j}\right)$ that maximizes the probability of reelection for any sequential rational $a^{S R}$. Then, the social welfare function $W$ to maximize is

$$
\begin{aligned}
& W\left(d^{A}, d^{B} \mid p^{A}, p^{B}\right)=E\left[\operatorname{Pr}\left\{R^{A}=0 ; g^{A}, l^{A} \mid \sigma^{A}=p^{A}\right\}\right]+E\left[\operatorname{Pr}\left\{R^{B}=0 ; g^{B}, l^{B} \mid \sigma^{B}=p^{B}\right\}\right] \propto \\
& \propto-\left[\gamma d^{A}+\frac{a \pi}{p^{A}+p^{A} d^{A}+1}+\frac{a(1-\pi)}{p^{A}+p^{A} d^{A}+2}+\gamma d^{B}+\frac{(1-a) \pi}{p^{B}+p^{B} d^{B}+1}+\frac{(1-a)(1-\pi)}{p^{B}+p^{B} d^{B}+2}\right] .
\end{aligned}
$$

Obviously the social welfare function depends also on the values of $a$, that are anticipated as sequentially rational. Since the efficiency problem is symmetric in $A$ and $B$, the possible solution satisfy the restriction $d^{E}=\left(d^{E, A}\left(p^{A}\right)=d^{E, B}\left(p^{B}\right)\right)$ where

$d^{E, j}\left(p^{j}\right) \in\left\{\left\{\begin{array}{ll}0 & \text { if } p^{j}=1 \\ 0 & \text { if } p^{j}=2\end{array},\left\{\begin{array}{ll}0 & \text { if } p^{j}=1 \\ 1 & \text { if } p^{j}=2\end{array},\left\{\begin{array}{ll}1 & \text { if } p^{j}=1 \\ 0 & \text { if } p^{j}=2\end{array},\left\{\begin{array}{lll}1 & \text { if } p^{j}=1 \\ 1 & \text { if } p^{j}=2\end{array}\right\}\right.\right.\right.\right.$.

Therefore, the possible efficient solution may be

$$
\begin{aligned}
& d^{E}=\left(d^{E, A}\left(p^{A}\right), d^{E, B}\left(p^{B}\right)\right) \in \\
& \in\left\{\left\{\begin{array}{ll}
(0,0) & \text { if } p^{j}=1 \\
(0,0) & \text { if } p^{j}=2
\end{array},\left\{\begin{array}{ll}
(0,0) & \text { if } p^{j}=1 \\
(1,1) & \text { if } p^{j}=2
\end{array},\left\{\begin{array}{ll}
(1,1) & \text { if } p^{j}=1 \\
(0,0) & \text { if } p^{j}=2
\end{array},\left\{\begin{array}{ll}
(1,1) & \text { if } p^{j}=1 \\
(1,1) & \text { if } p^{j}=2
\end{array}\right\}\right.\right.\right.\right.
\end{aligned}
$$

that to simplify we will denote omitting the second column. Hence

$$
\begin{gathered}
d^{E}=\left(d^{E, A}\left(p^{A}\right)=d^{E, B}\left(p^{B}\right)\right) \in \\
\in \arg \min \left\{E \left[W\left(\left\{\begin{array}{l}
(0,0) \\
(0,0)
\end{array}\right)\right], E\left[W\left(\left\{\begin{array}{l}
(0,0) \\
(1,1)
\end{array}\right)\right], E\left[W\left(\left\{\begin{array}{l}
(1,1) \\
(0,0)
\end{array}\right)\right], E\left[W\left(\left\{\begin{array}{l}
(1,1) \\
(1,1)
\end{array}\right)\right]\right\}\right.\right.\right.\right.
\end{gathered}
$$

where

$W\left(\left\{\begin{array}{c}(0,0) \\ (0,0)\end{array}\right)= \begin{cases}\frac{a^{S R} \pi}{2}+\frac{a^{S R}(1-\pi)}{3}+\frac{\left(1-a^{S R}\right) \pi}{2}+\frac{\left(1-a^{S R}\right)(1-\pi)}{3} & \text { if } \quad\left(p^{A}=1, p^{B}=1\right) \\ \frac{a^{S R} \pi}{3}+\frac{a^{S R}(1-\pi)}{4}+\frac{\left(1-a^{S R}\right) \pi}{2}+\frac{\left(1-a^{S R}\right)(1-\pi)}{3} & \text { if }\left(p^{A}=2, p^{B}=1\right) \\ \frac{a^{S R} \pi}{2}+\frac{a^{S R}(1-\pi)}{3}+\frac{\left(1-a^{S R}\right) \pi}{3}+\frac{\left(1-a^{S R}\right)(1-\pi)}{4} & \text { if }\left(p^{A}=1, p^{B}=2\right) \\ \frac{a^{S R} \pi}{3}+\frac{a^{S R}(1-\pi)}{4}+\frac{\left(1-a^{S R}\right) \pi}{3}+\frac{\left(1-a^{S R}\right)(1-\pi)}{4} & \text { if } \quad\left(p^{A}=2, p^{B}=2\right)\end{cases}\right.$ 


$$
\begin{aligned}
& W\left(\left\{\begin{array}{c}
(0,0) \\
(1,1)
\end{array}\right)=\left\{\begin{array}{cll}
\frac{a^{S R} \pi}{2}+\frac{a^{S R}(1-\pi)}{3}+\frac{\left(1-a^{S R}\right) \pi}{2}+\frac{\left(1-a^{S R}\right)(1-\pi)}{3} & \text { if } \quad\left(p^{A}=1, p^{B}=1\right) \\
\gamma+\frac{a^{S R} \pi}{5}+\frac{a^{S R}(1-\pi)}{6}+\frac{\left(1-a^{S R}\right) \pi}{2^{S R}}+\frac{\left(1-a^{S R}\right)(1-\pi)}{3} & \text { if } \quad\left(p^{A}=2, p^{B}=1\right) \\
\frac{a^{S R} \pi}{2}+\frac{a^{S R}(1-\pi)}{3}+\gamma+\frac{\left(1-a^{S R}\right) \pi}{5}+\frac{\left(1-a^{S R}\right)(1-\pi)}{6} & \text { if } \quad\left(p^{A}=1, p^{B}=2\right) \\
\gamma+\frac{a^{S R} \pi}{5}+\frac{a^{S R}(1-\pi)}{6}+\gamma+\frac{\left(1-a^{S R}\right) \pi}{5}+\frac{\left(1-a^{S R}\right)(1-\pi)}{6} & \text { if } \quad\left(p^{A}=2, p^{B}=2\right)
\end{array}\right.\right. \\
& W\left(\left\{\begin{array}{c}
(1,1) \\
(0,0)
\end{array}\right)=\left\{\begin{array}{cll}
\gamma+\frac{a^{S R} \pi}{3}+\frac{a^{S R}(1-\pi)}{4}+\gamma+\frac{\left(1-a^{S R}\right) \pi}{3}+\frac{\left(1-a^{S R}\right)(1-\pi)}{4} & \text { if } \quad\left(p^{A}=1, p^{B}=1\right) \\
\frac{a^{S R} \pi}{3}+\frac{a^{S R}(1-\pi)}{4}+\gamma+\frac{\left(1-a^{S R}\right) \pi}{3}+\frac{\left(1-a^{S R}\right)(1-\pi)}{3^{4}} & \text { if } \quad\left(p^{A}=2, p^{B}=1\right) \\
\gamma+\frac{a^{S R} \pi}{3}+\frac{a^{S R}(1-\pi)}{4}+\frac{\left(1-a^{S R}\right) \pi}{3}+\frac{\left(1-a^{S R}\right)(1-\pi)}{4} & \text { if } \quad\left(p^{A}=1, p^{B}=2\right) \\
\frac{a^{S R} \pi}{3}+\frac{a^{S R}(1-\pi)}{4}+\frac{\left(1-a^{S R}\right) \pi}{3}+\frac{\left(1-a^{S R}\right)(1-\pi)}{4} & \text { if } \quad\left(p^{A}=2, p^{B}=2\right)
\end{array}\right.\right. \\
& W\left(\left\{\begin{array}{c}
(1,1) \\
(1,1)
\end{array}\right)=\left\{\begin{array}{lll}
\gamma+\frac{a^{S R} \pi}{3}+\frac{a^{S R}(1-\pi)}{4}+\gamma+\frac{\left(1-a^{S R}\right) \pi}{3^{S R}}+\frac{\left(1-a^{S R}\right)(1-\pi)}{\left(1-a^{S}\right)(1-\pi)} & \text { if } \quad\left(p^{A}=1, p^{B}=1\right) \\
\left.\gamma+\frac{a^{S R} \pi}{5}+\frac{a^{S R}(1-\pi)}{6}+\gamma+\frac{\left(1-a^{S R}\right)}{3}+\frac{\left(1-a^{A}=2, p^{B}=1\right)}{3^{4}}\right) & \text { if }(1-\pi) \\
\gamma+\frac{a^{S R} \pi}{3}+\frac{a^{S R}(1-\pi)}{4}+\gamma+\frac{\left(1-a^{S R}\right) \pi}{5}+\frac{\left(1-a^{S R}\right)(1-\pi}{5^{6}} & \text { if } \quad\left(p^{A}=1, p^{B}=2\right) \\
\gamma+\frac{a^{S R} \pi}{5}+\frac{a^{S R}(1-\pi)}{6}+\gamma+\frac{\left(1-a^{S R}\right) \pi}{5}+\frac{\left(1-a^{S R}\right)(1-\pi)}{6} & \text { if } \quad\left(p^{A}=2, p^{B}=2\right)
\end{array}\right.\right.
\end{aligned}
$$

Now we should consider the terrorists' sequential rational choice $a^{S R}$. From the calculation of Appendix A, assuming Passive Updating (PU)

1.

$$
W\left(\left\{\begin{array}{c}
(0,0) \\
(0,0)
\end{array}\right)=\left\{\begin{array}{ccc}
\frac{\pi}{2}+\frac{1-\pi}{3} & \text { if } & \left(p^{A}=1, p^{B}=1\right) \\
\frac{\pi}{2}+\frac{1-\pi}{3}-\frac{a^{*} \pi}{6}-\frac{a^{*}(1-\pi)}{12} & \text { if } & \left(p^{A}=2, p^{B}=1\right) \\
\frac{\pi}{3}+\frac{1-\pi}{4}+\frac{a^{*} \pi}{6}+\frac{a^{*}(1-\pi)}{12} & \text { if } & \left(p^{A}=1, p^{B}=2\right) \\
\frac{\pi}{3}+\frac{1-\pi}{4} & \text { if } & \left(p^{A}=2, p^{B}=2\right)
\end{array}\right.\right.
$$

2 .

$$
W\left(\left\{\begin{array}{c}
(0,0) \\
(1,1)
\end{array}\right)=\left\{\begin{array}{ccc}
\frac{\pi}{2}+\frac{1-\pi}{3} & \text { if } \quad\left(p^{A}=1, p^{B}=1\right) \\
\gamma+\frac{\pi}{5}+\frac{1-\pi}{6} & \text { if } & \left(p^{A}=2, p^{B}=1\right) \\
\gamma+\frac{\pi}{5}+\frac{1-\pi}{6} & \text { if } & \left(p^{A}=1, p^{B}=2\right) \\
2 \gamma+\frac{\pi}{5}+\frac{1-\pi}{6} & \text { if } & \left(p^{A}=2, p^{B}=2\right)
\end{array}\right.\right.
$$

3.

$$
W\left(\left\{\begin{array}{c}
(1,1) \\
(0,0)
\end{array}\right)=\left\{\begin{array}{ccc}
2 \gamma+\frac{\pi}{3}+\frac{1-\pi}{4} & \text { if } & \left(p^{A}=1, p^{B}=1\right) \\
\gamma+\frac{\pi}{3}+\frac{1-\pi}{4} & \text { if } & \left(p^{A}=2, p^{B}=1\right) \\
\gamma+\frac{\pi}{3}+\frac{(1-\pi)}{4} & \text { if } & \left(p^{A}=1, p^{B}=2\right) \\
\frac{\pi}{3}+\frac{1-\pi}{4} & \text { if } & \left(p^{A}=2, p^{B}=2\right)
\end{array}\right.\right.
$$

4.

$$
W\left(\left\{\begin{array}{c}
(1,1) \\
(1,1)
\end{array}\right)=\left\{\begin{array}{ccc}
2 \gamma+\frac{\pi}{3}+\frac{1-\pi}{4} & \text { if } \quad\left(p^{A}=1, p^{B}=1\right) \\
2 \gamma+\frac{\pi}{3}+\frac{1-\pi}{4}-\frac{2 a^{*} \pi}{15}-\frac{a^{*}(1-\pi)}{12} & \text { if } \quad\left(p^{A}=2, p^{B}=1\right) \\
2 \gamma+\frac{\pi}{5}+\frac{1-\pi}{6}+\frac{2 a^{*} \pi}{15}+\frac{a^{*}(1-\pi)}{12} & \text { if } \quad\left(p^{A}=1, p^{B}=2\right) \\
2 \gamma+\frac{\pi}{5}+\frac{1-\pi}{6} & \text { if } \quad\left(p^{A}=2, p^{B}=2\right)
\end{array}\right.\right.
$$


Thus:

1.

$$
E\left[W\left(\left\{\begin{array}{c}
(0,0) \\
(0,0)
\end{array}\right)\right]=\pi\left(\frac{\pi}{2}+\frac{1-\pi}{3}\right)+(1-\pi)\left(\frac{\pi}{3}+\frac{1-\pi}{4}\right)\right.
$$

2.

$$
E\left[W\left(\left\{\begin{array}{l}
(0,0) \\
(1,1)
\end{array}\right)\right]=2(1-\pi) \gamma+\pi^{2}\left(\frac{\pi}{2}+\frac{1-\pi}{3}\right)+\left(1-\pi^{2}\right)\left(\frac{\pi}{5}+\frac{1-\pi}{6}\right)\right.
$$

3.

$$
E\left[W\left(\left\{\begin{array}{l}
(1,1) \\
(0,0)
\end{array}\right)\right]=2 \pi \gamma+\frac{\pi}{3}+\frac{1-\pi}{4}\right.
$$

4.

$$
E\left[W\left(\left\{\begin{array}{c}
(1,1) \\
(1,1)
\end{array}\right)\right]=2 \gamma+\pi\left(\frac{\pi}{3}+\frac{1-\pi}{4}\right)+(1-\pi)\left(\frac{\pi}{5}+\frac{1-\pi}{6}\right)\right.
$$

Note that

1.

$$
E\left[W\left(\left\{\begin{array}{c}
(1,1) \\
(0,0)
\end{array}\right)\right] \geq \min \left\{E \left[W\left(\left\{\begin{array}{c}
(0,0) \\
(0,0)
\end{array}\right)\right], E\left[W\left(\left\{\begin{array}{c}
(0,0) \\
(1,1)
\end{array}\right)\right], E\left[W\left(\left\{\begin{array}{c}
(1,1) \\
(1,1)
\end{array}\right)\right]\right\}\right.\right.\right.\right.
$$

2.

$$
E\left[W\left(\left\{\begin{array}{c}
(0,0) \\
(0,0)
\end{array}\right)\right] \leq E\left[W\left(\left\{\begin{array}{c}
(0,0) \\
(1,1)
\end{array}\right)\right] \Leftrightarrow \pi\left(\frac{3 \pi}{20}+\frac{1-\pi}{12}\right)+\frac{\pi}{15}+\frac{1-\pi}{24} \leq \gamma\right.\right.
$$

3.

$$
E\left[W\left(\left\{\begin{array}{c}
(1,1) \\
(1,1)
\end{array}\right)\right] \leq E\left[W\left(\left\{\begin{array}{c}
(0,0) \\
(1,1)
\end{array}\right)\right] \Leftrightarrow \gamma \leq \pi\left(\frac{\pi}{4}+\frac{1-\pi}{6}\right)+(1-\pi)\left(\frac{\pi}{10}+\frac{1-\pi}{12}\right)-\left(\frac{\pi}{6}+\frac{1-1}{8}\right.\right.\right.
$$

Hence, we can conclude with the following lemma.

Lemma 2 When there is private information, the countries efficient defensive policies are

1.

$$
\gamma \in\left[0, \pi\left(\frac{\pi}{4}+\frac{1-\pi}{6}\right)+(1-\pi)\left(\frac{\pi}{10}+\frac{1-\pi}{12}\right)-\left(\frac{\pi}{6}+\frac{1-\pi}{8}\right)\right] \Leftrightarrow d^{E, j}\left(p^{A}\right)=\left\{\begin{array}{lll}
1 & \text { if } & p^{j}=1 \\
1 & \text { if } & p^{j}=2
\end{array}\right.
$$

2.

$$
\begin{aligned}
\gamma & \in\left[\pi\left(\frac{\pi}{4}+\frac{1-\pi}{6}\right)+(1-\pi)\left(\frac{\pi}{10}+\frac{1-\pi}{12}\right)-\left(\frac{\pi}{6}+\frac{1-\pi}{8}\right), \pi\left(\frac{3 \pi}{20}+\frac{1-\pi}{12}\right)+\frac{\pi}{15}+\frac{1-\pi}{24}\right] \Leftrightarrow \\
& \Leftrightarrow d^{E, j}\left(p^{A}\right)= \begin{cases}0 & \text { if } p^{j}=1 \\
1 & \text { if } p^{j}=2\end{cases}
\end{aligned}
$$

3.

$$
\gamma \in\left[\pi\left(\frac{3 \pi}{20}+\frac{1-\pi}{12}\right)+\frac{\pi}{15}+\frac{1-\pi}{24}, 1\right] \Leftrightarrow d^{E, j}\left(p^{A}\right)=\left\{\begin{array}{lll}
0 & \text { if } & p^{j}=1 \\
0 & \text { if } & p^{j}=2
\end{array}\right.
$$




\subsection{The Countries Efficient Choices with Common Infor- mation}

When there is common information, $\sigma^{j}\left(p^{j}, p^{-j}\right)=\left(p^{j}, p^{-j}\right)$. The efficient outcome for the government and the citizens of country $j$ should consider this information, hence it is given by the choice $\delta^{E, j}\left(p^{A}, p^{B}\right)$ that maximizes the probability of reelection for any sequential rational $a^{S R}$. Then, the social welfare function $\widehat{W}$ to maximize is

$$
\begin{aligned}
& \widehat{W}=E[\operatorname{Pr}\left.\left\{R^{A}=0 ; g^{A}, l^{A} \mid \sigma^{A}=p^{A}, p^{B}\right\}\right]+E\left[\operatorname{Pr}\left\{R^{B}=0 ; g^{B}, l^{B} \mid \sigma^{B}=p^{A}, p^{B}\right\}\right] \propto \\
& \propto-\left[\gamma d^{A}+\frac{a}{p^{A}+p^{B}+p^{A} d^{A}}+\gamma d^{B}+\frac{(1-a)}{p^{A}+p^{B}+p^{B} d^{B}}\right] .
\end{aligned}
$$

Then the efficient choices are the solution of the following minimization problem

$d^{E}=\left(d^{E, A}\left(p^{A}, p^{B}\right), d^{E, B}\left(p^{A}, p^{B}\right)\right) \in \arg \min \left\{\gamma d^{A}+\frac{a^{S R}}{p^{A}+p^{B}+p^{A} d^{A}}+\gamma d^{B}+\frac{\left(1-a^{S R}\right)}{p^{A}+p^{B}+p^{B} d^{B}}\right\}$.

Since the efficiency problem is symmetric in $A$ and $B$, the possible efficient solution are

1.

$$
d^{E}=\left(d^{E, A}\left(p^{A}\right), d^{E, B}\left(p^{B}\right) \mid(1,1)\right) \in\{(0,0),(1,1)\}
$$

2.

$$
d^{E}=\left(d^{E, A}\left(p^{A}\right), d^{E, B}\left(p^{B}\right) \mid(2,1)\right) \in\{(0,0),(1,0)(0,1),(1,1)\}
$$

3.

$$
d^{E}=\left(d^{E, A}\left(p^{A}\right), d^{E, B}\left(p^{B}\right) \mid(1,2)\right) \in\{(0,0),(0,1)(1,0),(1,1)\}
$$

4.

$$
d^{E}=\left(d^{E, A}\left(p^{A}\right), d^{E, B}\left(p^{B}\right) \mid(2,2)\right) \in\{(0,0),(1,1)\} .
$$

where to simplify we omitted the second column. Thus

1. when $\left(p^{A}=1, p^{B}=1\right)$, then $W(0,0)=\frac{1}{2} \quad \& \quad W(1,1)=2 \gamma+\frac{1}{3}$ hence

$$
W(0,0) \leq W(1,1) \Leftrightarrow \frac{1}{2} \leq 2 \gamma+\frac{1}{3} \Leftrightarrow \frac{1}{12} \leq \gamma \Rightarrow \delta^{E}(1,1)= \begin{cases}(0,0) & \text { if } \gamma \in\left[\frac{1}{12}, 1\right] \\ (1,1) & \text { if } \gamma \in\left[0, \frac{1}{12}\right]\end{cases}
$$

2. when $\left(p^{A}=2, p^{B}=1\right)$, then $W(0,0)=\frac{1}{3} \quad \& \quad W(1,1)=2 \gamma+\frac{1}{4}-\frac{a^{*}}{20}$, $W(1,0)=\gamma+\frac{1}{3}-\frac{2 a^{*}}{15}, \quad W(0,1)=\gamma+\frac{1}{4}+\frac{a^{*}}{12}$. Suppose $a^{*}=\frac{1}{2}$, then $W(1,0) \leq W(0,1) \Leftrightarrow 0 \leq \gamma, W(0,0) \leq W(1,0) \Leftrightarrow \frac{1}{15} \leq \gamma, W(0,0) \leq$ $W(1,0) \Leftrightarrow \frac{1}{15} \leq \gamma, W(1,0) \leq W(1,1) \Leftrightarrow \frac{1}{24} \leq \gamma$ so that

$$
\delta^{E}(2,1)= \begin{cases}(0,0) & \text { if } \gamma \in\left[\frac{1}{15}, 1\right] \\ (1,0) & \text { if } \gamma \in\left[\frac{1}{24}, \frac{1}{15}\right] \\ (1,1) & \text { if } \gamma \in\left[0, \frac{1}{24}\right]\end{cases}
$$


3. when $\left(p^{A}=1, p^{B}=2\right)$, then $W(0,0)=\frac{1}{3}, \quad W(1,1)=2 \gamma+\frac{1}{5}+\frac{a^{*}}{20}$, $W(1,0)=\gamma+\frac{1}{3}-\frac{a^{*}}{12}, W(0,1)=\gamma+\frac{1}{5}+\frac{2 a^{*}}{15}$. To simplify suppose $a^{*}=\frac{1}{2}$, then $W(0,1) \leq W(1,0) \Leftrightarrow 0 \leq \gamma \quad \& \quad W(0,0) \leq W(0,1) \Leftrightarrow \frac{1}{15} \leq \gamma \quad \&$ $W(0,1) \leq W(1,1) \Leftrightarrow \frac{1}{24} \leq \gamma$ so that

$$
\delta^{E}(1,2)= \begin{cases}(0,0) & \text { if } \gamma \in\left[\frac{1}{15}, 1\right] \\ (0,1) & \text { if } \gamma \in\left[\frac{1}{24}, \frac{1}{15}\right] \\ (1,1) & \text { if } \gamma \in\left[0, \frac{1}{24}\right]\end{cases}
$$

4. when $\left(p^{A}=2, p^{B}=2\right)$, then $W(0,0)=\frac{1}{4} \quad \& \quad W(1,1)=2 \gamma+\frac{1}{6}$ hence $W(0,0) \leq W(1,1) \Leftrightarrow \frac{1}{24} \leq \gamma$ so that

$$
\delta^{E}(1,1)= \begin{cases}(0,0) & \text { if } \gamma \in\left[\frac{1}{24}, 1\right] \\ (1,1) & \text { if } \gamma \in\left[0, \frac{1}{24}\right] .\end{cases}
$$

Hence, we can conclude with the following lemma.

Lemma 3 When there is common information, the countries efficient policies are

1.

$$
\gamma \in\left[\frac{1}{12}, 1\right] \Rightarrow d^{E}= \begin{cases}(0,0) & \text { if }\left(p^{A}, p^{B}\right)=(1,1) \\ (0,0) & \text { if }\left(p^{A}, p^{B}\right)=(2,1) \\ (0,0) & \text { if }\left(p^{A}, p^{B}\right)=(1,2) \\ (0,0) & \text { if }\left(p^{A}, p^{B}\right)=(2,2)\end{cases}
$$

2.

$$
\gamma \in\left[\frac{1}{15}, \frac{1}{12}\right] \Rightarrow d^{E}= \begin{cases}(1,1) & \text { if }\left(p^{A}, p^{B}\right)=(1,1) \\ (0,0) & \text { if }\left(p^{A}, p^{B}\right)=(2,1) \\ (0,0) & \text { if }\left(p^{A}, p^{B}\right)=(1,2) \\ (0,0) & \text { if }\left(p^{A}, p^{B}\right)=(2,2)\end{cases}
$$

3.

$$
\gamma \in\left[\frac{1}{24}, \frac{1}{15}\right] \Rightarrow d^{E}= \begin{cases}(1,1) & \text { if }\left(p^{A}, p^{B}\right)=(1,1) \\ (1,0) & \text { if }\left(p^{A}, p^{B}\right)=(2,1) \\ (0,1) & \text { if }\left(p^{A}, p^{B}\right)=(1,2) \\ (0,0) & \text { if }\left(p^{A}, p^{B}\right)=(2,2)\end{cases}
$$

4.

$$
\gamma \in\left[0, \frac{1}{24}\right] \Rightarrow d^{E}= \begin{cases}(1,1) & \text { if }\left(p^{A}, p^{B}\right)=(1,1) \\ (1,1) & \text { if }\left(p^{A}, p^{B}\right)=(2,1) \\ (1,1) & \text { if }\left(p^{A}, p^{B}\right)=(1,2) \\ (1,1) & \text { if }\left(p^{A}, p^{B}\right)=(2,2)\end{cases}
$$




\section{Appendix C: Perfect Bayesian Equilibria with Full Decentralization}

Let we distinguish four possible kind of PBE:

1. A and B separating, with four possible strategy profiles

2. A separating and B pooling, with four possible strategy profiles

3. A pooling and B separating, with four possible strategy profiles

4. A and B pooling, with four possible strategy profiles.

Hence, totally there are sixteen possible strategy profiles. To model out-of equilibrium beliefs, we assume Passive Updating (PU).

\section{A and B separating}

\section{Case 1.1}

$$
\begin{gathered}
\delta^{A}\left(p^{A}\right)=\left\{\begin{array}{ll}
0 & \text { if } p^{A}=1 \\
1 & \text { if } p^{A}=2
\end{array} \text { and } \delta^{B}\left(p^{B}\right)= \begin{cases}0 & \text { if } p^{B}=1 \\
1 & \text { if } p^{B}=2\end{cases} \right. \\
\mu^{A}=\mu\left(p^{A}=1 \mid d^{A}\right)=\left\{\begin{array}{ll}
1 & \text { if } d^{A}=0 \\
0 & \text { if } d^{A}=1
\end{array} \text { and } \mu^{B}=\mu\left(p^{B}=1 \mid d^{B}\right)= \begin{cases}1 & \text { if } d^{B}=0 \\
0 & \text { if } d^{B}=1\end{cases} \right.
\end{gathered}
$$

- when $\left(d^{A}=0, d^{B}=0\right)$, then

$$
E\left(\frac{p^{A} a}{p^{A}+p^{B}+p^{A} d^{A}}\right)+E\left(\frac{p^{B}(1-a)}{p^{A}+p^{B}+p^{B} d^{B}}\right)=\frac{a}{2}+\frac{(1-a)}{2}=\frac{1}{2} \Rightarrow a^{* A}\left(d^{A}=0, d^{B}=0\right) \in[0,1]
$$

- when $\left(d^{A}=0, d^{B}=1\right)$, then

$$
E\left(\frac{p^{A} a}{p^{A}+p^{B}+p^{A} d^{A}}\right)+E\left(\frac{p^{B}(1-a)}{p^{A}+p^{B}+p^{B} d^{B}}\right)=\frac{a}{3}+\frac{2(1-a)}{5}=\frac{6-a}{15} \Rightarrow a^{* A}\left(d^{A}=0, d^{B}=1\right)=0
$$

- when $\left(d^{A}=1, d^{B}=0\right)$, then

$$
E\left(\frac{p^{A} a}{p^{A}+p^{B}+p^{A} d^{A}}\right)+E\left(\frac{p^{B}(1-a)}{p^{A}+p^{B}+p^{B} d^{B}}\right)=\frac{2 a}{5}+\frac{(1-a)}{3}=\frac{a+5}{15} \Rightarrow a^{* A}\left(d^{A}=1, d^{B}=0\right)=1
$$

- when $\left(d^{A}=1, d^{B}=1\right)$, then

$$
E\left(\frac{p^{A} a}{p^{A}+p^{B}+p^{A} d^{A}}\right)+E\left(\frac{p^{B}(1-a)}{p^{A}+p^{B}+p^{B} d^{B}}\right)=\frac{a}{3}+\frac{(1-a)}{3}=\frac{1}{3} \Rightarrow a^{* A}\left(d^{A}=1, d^{B}=1\right) \in[0,1]
$$

To conclude

$$
a^{* A}\left(d^{A}, d^{B}\right) \in\left\{\begin{array}{cc}
{[0,1]} & \text { if } \quad\left(d^{A}=0, d^{B}=0\right) \\
\{0\} & \text { if } \quad\left(d^{A}=0, d^{B}=1\right) \\
\{1\} & \text { if } \quad\left(d^{A}=1, d^{B}=0\right) \\
{[0,1]} & \text { if } \quad\left(d^{A}=1, d^{B}=1\right)
\end{array}\right.
$$


First stage: the governments' rational choices

$$
\begin{gathered}
\delta^{A}\left(p^{A}\right)=\left\{\begin{array}{ll}
0 & \text { if } p^{A}=1 \\
1 & \text { if } p^{A}=2
\end{array} \text { and } \delta^{B}\left(p^{B}\right)=\left\{\begin{array}{lll}
0 & \text { if } p^{B}=1 \\
1 & \text { if } p^{B}=2
\end{array}\right.\right. \\
a^{* A}\left(d^{A}, d^{B}\right) \in\left\{\begin{array}{cc}
{[0,1]} & \text { if }\left(d^{A}=0, d^{B}=0\right) \\
\{0\} & \text { if }\left(d^{A}=0, d^{B}=1\right) \\
\{1\} & \text { if }\left(d^{A}=1, d^{B}=0\right) \\
{[0,1]} & \text { if }\left(d^{A}=1, d^{B}=1\right)
\end{array}\right.
\end{gathered}
$$

are part of a PBE if and only if

$$
\begin{aligned}
& \begin{cases}-\left(\gamma d^{* A}+\frac{a^{* A}}{p^{A}+1+p^{A} d^{* A}}\right) \pi-\left(\gamma d^{* A}+\frac{a^{* A}}{p^{A}+2+p^{A} d^{* A}}\right)(1-\pi) \geq & \text { if } p^{A}=1 \\
\geq-\left(\gamma d^{A}+\frac{a^{* A}}{p^{A}+1+p^{A} d^{A}}\right) \pi-\left(\gamma d^{A}+\frac{a^{* A}}{p^{A}+2+p^{A} d^{A}}\right)(1-\pi) & \\
-\left(\gamma d^{* A}+\frac{a^{* A}}{p^{A}+1+p^{A} d^{* A}}\right) \pi-\left(\gamma d^{* A}+\frac{a^{* A}}{p^{A}+2+p^{A} d^{* A}}\right)(1-\pi) \geq & \text { if } p^{A}=2 \\
\geq-\left(\gamma d^{A}+\frac{a^{* A}}{p^{A}+1+p^{A} d^{A}}\right) \pi-\left(\gamma d^{A}+\frac{a^{* A}}{p^{A}+2+p^{A} d^{A}}\right)(1-\pi) & \end{cases} \\
& \left\{\begin{array}{l}
-\left(\gamma d^{* B}+\frac{1-a^{* A}}{p^{B}+1+p^{B} d^{* B}}\right) \pi-\left(\gamma d^{* B}+\frac{1-a^{* A}}{p^{B}+2+p^{B} d^{* B}}\right)(1-\pi) \geq \\
\geq-\left(\gamma d^{B}+\frac{1-a^{* A}}{p^{B}+1+p^{B} d^{B}}\right) \pi-\left(\gamma d^{B}+\frac{1-a^{* A}}{p^{B}+2+p^{B} d^{B}}\right)(1-\pi) \\
-\left(\gamma d^{* B}+\frac{1-a^{* A}}{p^{B}+1+p^{B} d^{* B}}\right) \pi-\left(\gamma d^{* B}+\frac{1-a^{* A}}{p^{B}+2+p^{B} d^{* B}}\right)(1-\pi) \geq \\
\geq-\left(\gamma d^{B}+\frac{1-a^{* A}}{p^{B}+1+p^{B} d^{B}}\right) \pi-\left(\gamma d^{B}+\frac{1-a^{* A}}{p^{B}+2+p^{B} d^{B}}\right)(1-\pi)
\end{array}\right.
\end{aligned}
$$

which are impossible because the four restrictions are mutually inconsistent.

\section{Case 1.2}

$$
\begin{gathered}
\delta^{A}\left(p^{A}\right)=\left\{\begin{array}{ll}
0 & \text { if } p^{A}=1 \\
1 & \text { if } p^{A}=2
\end{array} \text { and } \delta^{B}\left(p^{B}\right)=\left\{\begin{array}{lll}
1 & \text { if } p^{B}=1 \\
0 & \text { if } p^{B}=2
\end{array}\right.\right. \\
\mu^{A}=\mu\left(p^{A}=1 \mid d^{A}\right)=\left\{\begin{array}{ll}
1 & \text { if } d^{A}=0 \\
0 & \text { if } d^{A}=1
\end{array} \text { and } \mu^{B}=\mu\left(p^{B}=1 \mid d^{B}\right)= \begin{cases}0 & \text { if } d^{B}=0 \\
1 & \text { if } d^{B}=1\end{cases} \right.
\end{gathered}
$$

- when $\left(d^{A}=0, d^{B}=0\right)$, then

$$
E\left(\frac{p^{A} a}{p^{A}+p^{B}+p^{A} d^{A}}\right)+E\left(\frac{p^{B}(1-a)}{p^{A}+p^{B}+p^{B} d^{B}}\right)=\frac{2-a}{3} \Rightarrow a^{* A}\left(d^{A}=0, d^{B}=0\right)=0
$$

- when $\left(d^{A}=0, d^{B}=1\right)$, then

$$
E\left(\frac{p^{A} a}{p^{A}+p^{B}+p^{A} d^{A}}\right)+E\left(\frac{p^{B}(1-a)}{p^{A}+p^{B}+p^{B} d^{B}}\right)=\frac{a+2}{6} \Rightarrow a^{* A}\left(d^{A}=0, d^{B}=1\right)=1
$$

- when $\left(d^{A}=1, d^{B}=0\right)$, then

$$
E\left(\frac{p^{A} a}{p^{A}+p^{B}+p^{A} d^{A}}\right)+E\left(\frac{p^{B}(1-a)}{p^{A}+p^{B}+p^{B} d^{B}}\right)=\frac{3-a}{6} \Rightarrow a^{* A}\left(d^{A}=1, d^{B}=0\right)=0
$$


- when $\left(d^{A}=1, d^{B}=1\right)$, then

$$
E\left(\frac{p^{A} a}{p^{A}+p^{B}+p^{A} d^{A}}\right)+E\left(\frac{p^{B}(1-a)}{p^{A}+p^{B}+p^{B} d^{B}}\right)=\frac{3 a+5}{20} \Rightarrow a^{* A}\left(d^{A}=1, d^{B}=1\right)=1
$$

To conclude

$$
a^{* A}\left(d^{A}, d^{B}\right) \in\left\{\begin{array}{lll}
\{0\} & \text { if } & \left(d^{A}=0, d^{B}=0\right) \\
\{1\} & \text { if } & \left(d^{A}=0, d^{B}=1\right) \\
\{0\} & \text { if } & \left(d^{A}=1, d^{B}=0\right) \\
\{1\} & \text { if } & \left(d^{A}=1, d^{B}=1\right)
\end{array}\right.
$$

First stage: the governments' rational choices

$$
\begin{gathered}
\delta^{A}\left(p^{A}\right)=\left\{\begin{array}{ll}
0 & \text { if } p^{A}=1 \\
1 & \text { if } p^{A}=2
\end{array} \text { and } \delta^{B}\left(p^{B}\right)=\left\{\begin{array}{lll}
1 & \text { if } p^{B}=1 \\
0 & \text { if } p^{B}=2
\end{array}\right.\right. \\
a^{* A}\left(d^{A}, d^{B}\right) \in \begin{cases}\{0\} & \text { if }\left(d^{A}=0, d^{B}=0\right) \\
\{1\} & \text { if }\left(d^{A}=0, d^{B}=1\right) \\
\{0\} & \text { if }\left(d^{A}=1, d^{B}=0\right) \\
\{1\} & \text { if }\left(d^{A}=1, d^{B}=1\right)\end{cases}
\end{gathered}
$$

are part of a PBE if and only if

$$
\begin{aligned}
& \begin{cases}-\left(\gamma d^{* A}+\frac{a^{* A}}{p^{A}+1+p^{A} d^{* A}}\right) \pi-\left(\gamma d^{* A}+\frac{a^{* A}}{p^{A}+2+p^{A} d^{* A}}\right)(1-\pi) \geq & \text { if } p^{A}=1 \\
\geq-\left(\gamma d^{A}+\frac{a^{* A}}{p^{A}+1+p^{A} d^{A}}\right) \pi-\left(\gamma d^{A}+\frac{a^{* A}}{p^{A}+2+p^{A} d^{A}}\right)(1-\pi) & \\
-\left(\gamma d^{* A}+\frac{a^{* A}}{p^{A}+1+p^{A} d^{* A}}\right) \pi-\left(\gamma d^{* A}+\frac{a^{* A}}{p^{A}+2+p^{A} d^{* A}}\right)(1-\pi) \geq & \text { if } p^{A}=2 \\
\geq-\left(\gamma d^{A}+\frac{a^{* A}}{p^{A}+1+p^{A} d^{A}}\right) \pi-\left(\gamma d^{A}+\frac{a^{* A}}{p^{A}+2+p^{A} d^{A}}\right)(1-\pi) & \end{cases} \\
& \left\{\begin{array}{l}
-\left(\gamma d^{* B}+\frac{1-a^{* A}}{p^{B}+1+p^{B} d^{* B}}\right) \pi-\left(\gamma d^{* B}+\frac{1-a^{* A}}{p^{B}+2+p^{B} d^{* B}}\right)(1-\pi) \geq \\
\geq-\left(\gamma d^{B}+\frac{1-a^{* A}}{p^{B}+1+p^{B} d^{B}}\right) \pi-\left(\gamma d^{B}+\frac{1-a^{* A}}{p^{B}+2+p^{B} d^{B}}\right)(1-\pi) \\
-\left(\gamma d^{* B}+\frac{1-a^{* A}}{p^{B}+1+p^{B} d^{* B}}\right) \pi-\left(\gamma d^{* B}+\frac{1-a^{* A}}{p^{B}+2+p^{B} d^{* B}}\right)(1-\pi) \geq \\
\geq-\left(\gamma d^{B}+\frac{1-a^{* A}}{p^{B}+1+p^{B} d^{B}}\right) \pi-\left(\gamma d^{B}+\frac{1-a^{* A}}{p^{B}+2+p^{B} d^{B}}\right)(1-\pi)
\end{array}\right.
\end{aligned}
$$

which are impossible because the four restrictions are mutually inconsistent.

\section{Case 1.3}

$$
\begin{gathered}
\delta^{A}\left(p^{A}\right)=\left\{\begin{array}{ll}
1 & \text { if } p^{A}=1 \\
0 & \text { if } p^{A}=2
\end{array} \text { and } \delta^{B}\left(p^{B}\right)= \begin{cases}0 & \text { if } p^{B}=1 \\
1 & \text { if } p^{B}=2\end{cases} \right. \\
\mu^{A}=\mu\left(p^{A}=1 \mid d^{A}\right)=\left\{\begin{array}{ll}
0 & \text { if } d^{A}=0 \\
1 & \text { if } d^{A}=1
\end{array} \text { and } \mu^{B}=\mu\left(p^{B}=1 \mid d^{B}\right)= \begin{cases}1 & \text { if } d^{B}=0 \\
0 & \text { if } d^{B}=1\end{cases} \right.
\end{gathered}
$$


- when $\left(d^{A}=0, d^{B}=0\right)$, then

$$
E\left(\frac{p^{A} a}{p^{A}+p^{B}+p^{A} d^{A}}\right)+E\left(\frac{p^{B}(1-a)}{p^{A}+p^{B}+p^{B} d^{B}}\right)=\frac{a+1}{3} \Rightarrow a^{* A}\left(d^{A}=0, d^{B}=0\right)=1
$$

- when $\left(d^{A}=0, d^{B}=1\right)$, then

$$
E\left(\frac{p^{A} a}{p^{A}+p^{B}+p^{A} d^{A}}\right)+E\left(\frac{p^{B}(1-a)}{p^{A}+p^{B}+p^{B} d^{B}}\right)=\frac{a+2}{6} \Rightarrow a^{* A}\left(d^{A}=0, d^{B}=1\right)=1
$$

- when $\left(d^{A}=1, d^{B}=0\right)$, then

$$
E\left(\frac{p^{A} a}{p^{A}+p^{B}+p^{A} d^{A}}\right)+E\left(\frac{p^{B}(1-a)}{p^{A}+p^{B}+p^{B} d^{B}}\right)=\frac{3-a}{6} \Rightarrow a^{* A}\left(d^{A}=1, d^{B}=0\right)=0
$$

- when $\left(d^{A}=1, d^{B}=1\right)$, then

$$
E\left(\frac{p^{A} a}{p^{A}+p^{B}+p^{A} d^{A}}\right)+E\left(\frac{p^{B}(1-a)}{p^{A}+p^{B}+p^{B} d^{B}}\right)=\frac{8-3 a}{20} \Rightarrow a^{* A}\left(d^{A}=1, d^{B}=1\right)=0
$$

To conclude

$$
a^{* A}\left(d^{A}, d^{B}\right) \in\left\{\begin{array}{lll}
\{1\} & \text { if } & \left(d^{A}=0, d^{B}=0\right) \\
\{1\} & \text { if } & \left(d^{A}=0, d^{B}=1\right) \\
\{0\} & \text { if } & \left(d^{A}=1, d^{B}=0\right) \\
\{0\} & \text { if } & \left(d^{A}=1, d^{B}=1\right)
\end{array}\right.
$$

First stage: the governments' rational choices

$$
\begin{gathered}
\delta^{A}\left(p^{A}\right)=\left\{\begin{array}{ll}
1 & \text { if } p^{A}=1 \\
0 & \text { if } p^{A}=2
\end{array} \text { and } \delta^{B}\left(p^{B}\right)= \begin{cases}0 & \text { if } p^{B}=1 \\
1 & \text { if } p^{B}=2\end{cases} \right. \\
a^{* A}\left(d^{A}, d^{B}\right) \in \begin{cases}\{1\} & \text { if }\left(d^{A}=0, d^{B}=0\right) \\
\{1\} & \text { if }\left(d^{A}=0, d^{B}=1\right) \\
\{0\} & \text { if }\left(d^{A}=1, d^{B}=0\right) \\
\{0\} & \text { if }\left(d^{A}=1, d^{B}=1\right)\end{cases}
\end{gathered}
$$

are part of a PBE if and only if

$$
\left\{\begin{array}{cc}
-\left(\gamma d^{* A}+\frac{a^{* A}}{p^{A}+1+p^{A} d^{* A}}\right) \pi-\left(\gamma d^{* A}+\frac{a^{* A}}{p^{A}+2+p^{A} d^{* A}}\right)(1-\pi) \geq & \text { if } p^{A}=1 \\
\geq-\left(\gamma d^{A}+\frac{a^{* A}}{p^{A}+1+p^{A} d^{A}}\right) \pi-\left(\gamma d^{A}+\frac{a^{* A}}{p^{A}+2+p^{A} d^{A}}\right)(1-\pi) & \\
-\left(\gamma d^{* A}+\frac{a^{* A}}{p^{A}+1+p^{A} d^{* A}}\right) \pi-\left(\gamma d^{* A}+\frac{a^{* A}}{p^{A}+2+p^{A} d^{* A}}\right)(1-\pi) \geq & \text { if } p^{A}=2 \\
\geq-\left(\gamma d^{A}+\frac{a^{* A}}{p^{A}+1+p^{A} d^{A}}\right) \pi-\left(\gamma d^{A}+\frac{a^{* A}}{p^{A}+2+p^{A} d^{A}}\right)(1-\pi) &
\end{array}\right.
$$




$$
\left\{\begin{array}{l}
-\left(\gamma d^{* B}+\frac{1-a^{* A}}{p^{B}+1+p^{B} d^{* B}}\right) \pi-\left(\gamma d^{* B}+\frac{1-a^{* A}}{p^{B}+2+p^{B} d^{* B}}\right)(1-\pi) \geq \\
\geq-\left(\gamma d^{B}+\frac{1-a^{* A}}{p^{B}+1+p^{B} d^{B}}\right) \pi-\left(\gamma d^{B}+\frac{1-a^{* A}}{p^{B}+2+p^{B} d^{B}}\right)(1-\pi) \\
-\left(\gamma d^{* B}+\frac{1-a^{* A}}{p^{B}+1+p^{B} d^{* B}}\right) \pi-\left(\gamma d^{* B}+\frac{1-a^{* A}}{p^{B}+2+p^{B} d^{* B}}\right)(1-\pi) \geq \\
\geq-\left(\gamma d^{B}+\frac{1-a^{* A}}{p^{B}+1+p^{B} d^{B}}\right) \pi-\left(\gamma d^{B}+\frac{1-a^{* A}}{p^{B}+2+p^{B} d^{B}}\right)(1-\pi)
\end{array}\right.
$$

which are impossible.

\section{Case 1.4}

$$
\begin{gathered}
\delta^{A}\left(p^{A}\right)=\left\{\begin{array}{ll}
1 & \text { if } p^{A}=1 \\
0 & \text { if } p^{A}=2
\end{array} \text { and } \delta^{B}\left(p^{B}\right)= \begin{cases}1 & \text { if } p^{B}=1 \\
0 & \text { if } p^{B}=2\end{cases} \right. \\
\mu^{A}=\mu\left(p^{A}=1 \mid d^{A}\right)=\left\{\begin{array}{ll}
0 & \text { if } d^{A}=0 \\
1 & \text { if } d^{A}=1
\end{array} \text { and } \mu^{B}=\mu\left(p^{B}=1 \mid d^{B}\right)= \begin{cases}0 & \text { if } d^{B}=0 \\
1 & \text { if } d^{B}=1\end{cases} \right.
\end{gathered}
$$

- when $\left(d^{A}=0, d^{B}=0\right)$, then

$$
E\left(\frac{p^{A} a}{p^{A}+p^{B}+p^{A} d^{A}}\right)+E\left(\frac{p^{B}(1-a)}{p^{A}+p^{B}+p^{B} d^{B}}\right)=\frac{1}{2} \Rightarrow a^{* A}\left(d^{A}=0, d^{B}=0\right) \in[0,1]
$$

- when $\left(d^{A}=0, d^{B}=1\right)$, then

$$
E\left(\frac{p^{A} a}{p^{A}+p^{B}+p^{A} d^{A}}\right)+E\left(\frac{p^{B}(1-a)}{p^{A}+p^{B}+p^{B} d^{B}}\right)=\frac{5 a+3}{12} \Rightarrow a^{* A}\left(d^{A}=0, d^{B}=1\right)=1
$$

- when $\left(d^{A}=1, d^{B}=0\right)$, then

$$
E\left(\frac{p^{A} a}{p^{A}+p^{B}+p^{A} d^{A}}\right)+E\left(\frac{p^{B}(1-a)}{p^{A}+p^{B}+p^{B} d^{B}}\right)=\frac{8-5 a}{12} \Rightarrow a^{* A}\left(d^{A}=1, d^{B}=0\right)=0
$$

- when $\left(d^{A}=1, d^{B}=1\right)$, then

$$
E\left(\frac{p^{A} a}{p^{A}+p^{B}+p^{A} d^{A}}\right)+E\left(\frac{p^{B}(1-a)}{p^{A}+p^{B}+p^{B} d^{B}}\right)=\frac{1}{3} \Rightarrow a^{* A}\left(d^{A}=1, d^{B}=1\right) \in[0,1]
$$

To conclude

$$
a^{* A}\left(d^{A}, d^{B}\right) \in\left\{\begin{array}{cc}
{[0,1]} & \text { if } \quad\left(d^{A}=0, d^{B}=0\right) \\
\{1\} & \text { if } \quad\left(d^{A}=0, d^{B}=1\right) \\
\{0\} & \text { if } \quad\left(d^{A}=1, d^{B}=0\right) \\
{[0,1]} & \text { if } \quad\left(d^{A}=1, d^{B}=1\right)
\end{array}\right.
$$


First stage: the governments' rational choices

$$
\begin{gathered}
\delta^{A}\left(p^{A}\right)=\left\{\begin{array}{ll}
1 & \text { if } p^{A}=1 \\
0 & \text { if } p^{A}=2
\end{array} \text { and } \delta^{B}\left(p^{B}\right)=\left\{\begin{array}{lll}
1 & \text { if } p^{B}=1 \\
0 & \text { if } p^{B}=2
\end{array}\right.\right. \\
a^{* A}\left(d^{A}, d^{B}\right) \in\left\{\begin{array}{cc}
{[0,1]} & \text { if }\left(d^{A}=0, d^{B}=0\right) \\
\{1\} & \text { if }\left(d^{A}=0, d^{B}=1\right) \\
\{0\} & \text { if }\left(d^{A}=1, d^{B}=0\right) \\
{[0,1]} & \text { if }\left(d^{A}=1, d^{B}=1\right)
\end{array}\right.
\end{gathered}
$$

are part of a PBE if and only if

$$
\begin{aligned}
& \left\{\begin{array}{l}
-\left(\gamma d^{* A}+\frac{a^{* A}}{p^{A}+1+p^{A} d^{* A}}\right) \pi-\left(\gamma d^{* A}+\frac{a^{* A}}{p^{A}+2+p^{A} d^{* A}}\right)(1-\pi) \geq \\
\geq-\left(\gamma d^{A}+\frac{a^{* A}}{p^{A}+1+p^{A} d^{A}}\right) \pi-\left(\gamma d^{A}+\frac{a^{* A}}{p^{A}+2+p^{A} d^{A}}\right)(1-\pi) \\
-\left(\gamma d^{* A}+\frac{a^{* A}}{p^{A}+1+p^{A} d^{* A}}\right) \pi-\left(\gamma d^{* A}+\frac{a^{* A}}{p^{A}+2+p^{A} d^{* A}}\right)(1-\pi) \geq \\
\geq-\left(\gamma d^{A}+\frac{a^{* A}}{p^{A}+1+p^{A} d^{A}}\right) \pi-\left(\gamma d^{A}+\frac{a^{* A}}{p^{A}+2+p^{A} d^{A}}\right)(1-\pi)
\end{array}\right. \\
& \left\{\begin{array}{l}
-\left(\gamma d^{* B}+\frac{1-a^{* A}}{p^{B}+1+p^{B} d^{* B}}\right) \pi-\left(\gamma d^{* B}+\frac{1-a^{* A}}{p^{B}+2+p^{B} d^{* B}}\right)(1-\pi) \geq \\
\geq-\left(\gamma d^{B}+\frac{1-a^{* A}}{p^{B}+1+p^{B} d^{B}}\right) \pi-\left(\gamma d^{B}+\frac{1-a^{* A}}{p^{B}+2+p^{B} d^{B}}\right)(1-\pi) \\
-\left(\gamma d^{* B}+\frac{1-a^{* A}}{p^{B}+1+p^{B} d^{* B}}\right) \pi-\left(\gamma d^{* B}+\frac{1-a^{* A}}{p^{B}+2+p^{B} d^{* B}}\right)(1-\pi) \geq \\
\geq-\left(\gamma d^{B}+\frac{1-a^{* A}}{p^{B}+1+p^{B} d^{B}}\right) \pi-\left(\gamma d^{B}+\frac{1-a^{* A}}{p^{B}+2+p^{B} d^{B}}\right)(1-\pi)
\end{array}\right.
\end{aligned}
$$

which for any $\pi \in(0,1)$ and $a^{* A} \in[0,1]$ are possible when

$$
\begin{aligned}
\gamma \in[ & {\left[\frac{1}{3} \pi+\frac{a^{*}}{4}-\frac{9 a^{*}}{20} \pi, \frac{1}{2} \pi+\frac{a^{*}}{3}-\frac{2 a^{*}}{3} \pi\right] \cap } \\
& \cap\left[-\frac{7}{60} \pi+\frac{1}{4}-\frac{a^{*}}{4}+\frac{9 a^{*}}{20} \pi,-\frac{1}{6} \pi+\frac{a^{*}}{3} \pi+\frac{1}{3}\right] .
\end{aligned}
$$

\section{A separating and B pooling}

\section{Case 2.1}

$$
\begin{gathered}
\delta^{A}\left(p^{A}\right)=\left\{\begin{array}{ll}
0 & \text { if } p^{A}=1 \\
1 & \text { if } p^{A}=2
\end{array} \text { and } \delta^{B}\left(p^{B}\right)=\left\{\begin{array}{lll}
0 & \text { if } p^{B}=1 \\
0 & \text { if } p^{B}=2
\end{array}\right.\right. \\
\mu^{A}=\mu\left(p^{A}=1 \mid d^{A}\right)=\left\{\begin{array}{ll}
1 & \text { if } d^{A}=0 \\
0 & \text { if } d^{A}=1
\end{array} \text { and } \mu^{B}=\mu\left(p^{B}=1 \mid d^{B}\right)= \begin{cases}\pi & \text { if } d^{B}=0 \\
\pi & \text { if } d^{B}=1\end{cases} \right.
\end{gathered}
$$

- when $\left(d^{A}=0, d^{B}=0\right)$, then

$$
E\left(\frac{p^{A} a}{p^{A}+p^{B}+p^{A} d^{A}}\right)+E\left(\frac{p^{B}(1-a)}{p^{A}+p^{B}+p^{B} d^{B}}\right)=\frac{2 a \pi+4-2 a-\pi}{6} \Rightarrow a^{* A}\left(d^{A}=0, d^{B}=1\right)=0
$$


- when $\left(d^{A}=0, d^{B}=1\right)$, then

$$
\begin{gathered}
E\left(\frac{p^{A} a}{p^{A}+p^{B}+p^{A} d^{A}}\right)+E\left(\frac{p^{B}(1-a)}{p^{A}+p^{B}+p^{B} d^{B}}\right)= \\
=\left(\frac{7 \pi-2}{30}\right) a+\frac{12-2 \pi}{30} \Rightarrow a^{* A}\left(d^{A}=0, d^{B}=1\right)= \begin{cases}1 & \pi \geq \frac{2}{7} \\
0 & \pi \leq \frac{2}{7}\end{cases}
\end{gathered}
$$

- when $\left(d^{A}=1, d^{B}=0\right)$, then

$$
E\left(\frac{p^{A} a}{p^{A}+p^{B}+p^{A} d^{A}}\right)+E\left(\frac{p^{B}(1-a)}{p^{A}+p^{B}+p^{B} d^{B}}\right)=\frac{(7 \pi-5) a}{30}+\frac{15-5 \pi}{30} \Rightarrow a^{* A}(1,0)= \begin{cases}1 & \pi \geq \frac{5}{7} \\ 0 & \pi \leq \frac{5}{7}\end{cases}
$$

- when $\left(d^{A}=1, d^{B}=1\right)$, then

$$
\begin{gathered}
E\left(\frac{p^{A} a}{p^{A}+p^{B}+p^{A} d^{A}}\right)+E\left(\frac{p^{B}(1-a)}{p^{A}+p^{B}+p^{B} d^{B}}\right)= \\
=\frac{2 a \pi}{5}+\frac{a(1-\pi)}{3}+\frac{(1-a) \pi}{4}+\frac{(1-a)(1-\pi)}{3} \Rightarrow a^{* A}(1,0)=1
\end{gathered}
$$

To conclude

- if $\pi \in\left[0, \frac{2}{7}\right]$

$$
a^{* A}\left(d^{A}, d^{B}\right) \in\left\{\begin{array}{lll}
\{0\} & \text { if } & \left(d^{A}=0, d^{B}=0\right) \\
\{0\} & \text { if } & \left(d^{A}=0, d^{B}=1\right) \\
\{0\} & \text { if } & \left(d^{A}=1, d^{B}=0\right) \\
\{1\} & \text { if } & \left(d^{A}=1, d^{B}=1\right)
\end{array}\right.
$$

- if $\pi \in\left[\frac{2}{7}, \frac{5}{7}\right]$

$$
a^{* A}\left(d^{A}, d^{B}\right) \in\left\{\begin{array}{lll}
\{0\} & \text { if } & \left(d^{A}=0, d^{B}=0\right) \\
\{1\} & \text { if } & \left(d^{A}=0, d^{B}=1\right) \\
\{0\} & \text { if } & \left(d^{A}=1, d^{B}=0\right) \\
\{1\} & \text { if } & \left(d^{A}=1, d^{B}=1\right)
\end{array}\right.
$$

- if $\pi \in\left[\frac{5}{7}, 1\right]$

$$
a^{* A}\left(d^{A}, d^{B}\right) \in\left\{\begin{array}{lll}
\{0\} & \text { if } & \left(d^{A}=0, d^{B}=0\right) \\
\{1\} & \text { if } & \left(d^{A}=0, d^{B}=1\right) \\
\{1\} & \text { if } & \left(d^{A}=1, d^{B}=0\right) \\
\{1\} & \text { if } & \left(d^{A}=1, d^{B}=1\right)
\end{array}\right.
$$

First stage: the governments' rational choices 
Case $\pi \in\left[0, \frac{2}{7}\right]$

$$
\begin{gathered}
\delta^{A}\left(p^{A}\right)=\left\{\begin{array}{ll}
0 & \text { if } p^{A}=1 \\
1 & \text { if } p^{A}=2
\end{array} \text { and } \delta^{B}\left(p^{B}\right)=\left\{\begin{array}{lll}
0 & \text { if } p^{B}=1 \\
0 & \text { if } p^{B}=2
\end{array}\right.\right. \\
a^{* A}\left(d^{A}, d^{B}\right) \in \begin{cases}\{0\} & \text { if }\left(d^{A}=0, d^{B}=0\right) \\
\{0\} & \text { if }\left(d^{A}=0, d^{B}=1\right) \\
\{0\} & \text { if }\left(d^{A}=1, d^{B}=0\right) \\
\{1\} & \text { if }\left(d^{A}=1, d^{B}=1\right)\end{cases}
\end{gathered}
$$

are part of a PBE if and only if

$$
\begin{aligned}
& \begin{cases}-\left(\gamma d^{* A}+\frac{a^{* A}}{p^{A}+1+p^{A} d^{* A}}\right) \pi-\left(\gamma d^{* A}+\frac{a^{* A}}{p^{A}+2+p^{A} d^{* A}}\right)(1-\pi) \geq & \text { if } p^{A}=1 \\
\geq-\left(\gamma d^{A}+\frac{a^{* A}}{p^{A}+1+p^{A} d^{A}}\right) \pi-\left(\gamma d^{A}+\frac{a^{* A}}{p^{A}+2+p^{A} d^{A}}\right)(1-\pi) & \\
-\left(\gamma d^{* A}+\frac{a^{* A}}{p^{A}+1+p^{A} d^{* A}}\right) \pi-\left(\gamma d^{* A}+\frac{a^{* A}}{p^{A}+2+p^{A} d^{* A}}\right)(1-\pi) \geq & \text { if } p^{A}=2 \\
\geq-\left(\gamma d^{A}+\frac{a^{* A}}{p^{A}+1+p^{A} d^{A}}\right) \pi-\left(\gamma d^{A}+\frac{a^{* A}}{p^{A}+2+p^{A} d^{A}}\right)(1-\pi) & \end{cases} \\
& \left\{\begin{array}{l}
-\left(\gamma d^{* B}+\frac{1-a^{* A}}{p^{B}+1+p^{B} d^{* B}}\right) \pi-\left(\gamma d^{* B}+\frac{1-a^{* A}}{p^{B}+2+p^{B} d^{* B}}\right)(1-\pi) \geq \\
\geq-\left(\gamma d^{B}+\frac{1-a^{* A}}{p^{B}+1+p^{B} d^{B}}\right) \pi-\left(\gamma d^{B}+\frac{1-a^{* A}}{p^{B}+2+p^{B} d^{B}}\right)(1-\pi) \\
-\left(\gamma d^{* B}+\frac{1-a^{* A}}{p^{B}+1+p^{B} d^{* B}}\right) \pi-\left(\gamma d^{* B}+\frac{1-a^{* A}}{p^{B}+2+p^{B} d^{* B}}\right)(1-\pi) \geq \\
\geq-\left(\gamma d^{B}+\frac{1-a^{* A}}{p^{B}+1+p^{B} d^{B}}\right) \pi-\left(\gamma d^{B}+\frac{1-a^{* A}}{p^{B}+2+p^{B} d^{B}}\right)(1-\pi)
\end{array}\right.
\end{aligned}
$$

which for any $\pi \in\left[0, \frac{2}{7}\right]$ and $a^{* A} \in[0,1]$ is impossible.

Case $\pi \in\left[\frac{2}{7}, \frac{5}{7}\right]$

$$
\begin{gathered}
\delta^{A}\left(p^{A}\right)=\left\{\begin{array}{ll}
0 & \text { if } p^{A}=1 \\
1 & \text { if } p^{A}=2
\end{array} \text { and } \delta^{B}\left(p^{B}\right)= \begin{cases}0 & \text { if } p^{B}=1 \\
0 & \text { if } p^{B}=2\end{cases} \right. \\
a^{* A}\left(d^{A}, d^{B}\right) \in \begin{cases}\{0\} & \text { if }\left(d^{A}=0, d^{B}=0\right) \\
\{1\} & \text { if }\left(d^{A}=0, d^{B}=1\right) \\
\{0\} & \text { if }\left(d^{A}=1, d^{B}=0\right) \\
\{1\} & \text { if }\left(d^{A}=1, d^{B}=1\right)\end{cases}
\end{gathered}
$$

are part of a PBE if and only if

$$
\begin{cases}-\left(\gamma d^{* A}+\frac{a^{* A}}{p^{A}+1+p^{A} d^{* A}}\right) \pi-\left(\gamma d^{* A}+\frac{a^{* A}}{p^{A}+2+p^{A} d^{* A}}\right)(1-\pi) \geq & \text { if } p^{A}=1 \\ \geq-\left(\gamma d^{A}+\frac{a^{* A}}{p^{A}+1+p^{A} d^{A}}\right) \pi-\left(\gamma d^{A}+\frac{a^{* A}}{p^{A}+2+p^{A} d^{A}}\right)(1-\pi) & \\ -\left(\gamma d^{* A}+\frac{a^{* A}}{p^{A}+1+p^{A} d^{* A}}\right) \pi-\left(\gamma d^{* A}+\frac{a^{* A}}{p^{A}+2+p^{A} d^{* A}}\right)(1-\pi) \geq & \text { if } p^{A}=2 \\ \geq-\left(\gamma d^{A}+\frac{a^{* A}}{p^{A}+1+p^{A} d^{A}}\right) \pi-\left(\gamma d^{A}+\frac{a^{* A}}{p^{A}+2+p^{A} d^{A}}\right)(1-\pi) & \end{cases}
$$




$$
\left\{\begin{array}{l}
-\left(\gamma d^{* B}+\frac{1-a^{* A}}{p^{B}+1+p^{B} d^{* B}}\right) \pi-\left(\gamma d^{* B}+\frac{1-a^{* A}}{p^{B}+2+p^{B} d^{* B}}\right)(1-\pi) \geq \\
\geq-\left(\gamma d^{B}+\frac{1-a^{* A}}{p^{B}+1+p^{B} d^{B}}\right) \pi-\left(\gamma d^{B}+\frac{1-a^{* A}}{p^{B}+2+p^{B} d^{B}}\right)(1-\pi) \\
-\left(\gamma d^{* B}+\frac{1-a^{* A}}{p^{B}+1+p^{B} d^{* B}}\right) \pi-\left(\gamma d^{* B}+\frac{1-a^{* A}}{p^{B}+2+p^{B} d^{* B}}\right)(1-\pi) \geq \\
\geq-\left(\gamma d^{B}+\frac{1-a^{* A}}{p^{B}+1+p^{B} d^{B}}\right) \pi-\left(\gamma d^{B}+\frac{1-a^{* A}}{p^{B}+2+p^{B} d^{B}}\right)(1-\pi)
\end{array}\right.
$$

which for any $\pi \in\left[\frac{2}{7}, \frac{5}{7}\right]$ and $a^{* A} \in[0,1]$ is impossible.

Case $\pi \in\left[\frac{5}{7}, 1\right]$

$$
\begin{gathered}
\delta^{A}\left(p^{A}\right)=\left\{\begin{array}{ll}
0 & \text { if } p^{A}=1 \\
1 & \text { if } p^{A}=2
\end{array} \text { and } \delta^{B}\left(p^{B}\right)= \begin{cases}0 & \text { if } p^{B}=1 \\
0 & \text { if } p^{B}=2\end{cases} \right. \\
a^{* A}\left(d^{A}, d^{B}\right) \in \begin{cases}\{0\} & \text { if }\left(d^{A}=0, d^{B}=0\right) \\
\{1\} & \text { if }\left(d^{A}=0, d^{B}=1\right) \\
\{1\} & \text { if }\left(d^{A}=1, d^{B}=0\right) \\
\{1\} & \text { if }\left(d^{A}=1, d^{B}=1\right)\end{cases}
\end{gathered}
$$

are part of a PBE if and only if

$$
\begin{aligned}
& \left\{\begin{array}{l}
-\left(\gamma d^{* A}+\frac{a^{* A}}{p^{A}+1+p^{A} d^{* A}}\right) \pi-\left(\gamma d^{* A}+\frac{a^{* A}}{p^{A}+2+p^{A} d^{* A}}\right)(1-\pi) \geq \\
\geq-\left(\gamma d^{A}+\frac{a^{* A}}{p^{A}+1+p^{A} d^{A}}\right) \pi-\left(\gamma d^{A}+\frac{a^{* A}}{p^{A}+2+p^{A} d^{A}}\right)(1-\pi) \\
-\left(\gamma d^{* A}+\frac{a^{* A}}{p^{A}+1+p^{A} d^{* A}}\right) \pi-\left(\gamma d^{* A}+\frac{a^{* A}}{p^{A}+2+p^{A} d^{* A}}\right)(1-\pi) \geq \\
\geq-\left(\gamma d^{A}+\frac{a^{* A}}{p^{A}+1+p^{A} d^{A}}\right) \pi-\left(\gamma d^{A}+\frac{a^{* A}}{p^{A}+2+p^{A} d^{A}}\right)(1-\pi)
\end{array}\right. \\
& \left\{\begin{array}{l}
-\left(\gamma d^{* B}+\frac{1-a^{* A}}{p^{B}+1+p^{B} d^{* B}}\right) \pi-\left(\gamma d^{* B}+\frac{1-a^{* A}}{p^{B}+2+p^{B} d^{* B}}\right)(1-\pi) \geq \\
\geq-\left(\gamma d^{B}+\frac{1-a^{* A}}{p^{B}+1+p^{B} d^{B}}\right) \pi-\left(\gamma d^{B}+\frac{1-a^{* A}}{p^{B}+2+p^{B} d^{B}}\right)(1-\pi) \\
-\left(\gamma d^{* B}+\frac{1-a^{* A}}{p^{B}+1+p^{B} d^{* B}}\right) \pi-\left(\gamma d^{* B}+\frac{1-a^{* A}}{p^{B}+2+p^{B} d^{* B}}\right)(1-\pi) \geq \\
\geq-\left(\gamma d^{B}+\frac{1-a^{* A}}{p^{B}+1+p^{B} d^{B}}\right) \pi-\left(\gamma d^{B}+\frac{1-a^{*}}{p^{B}+2+p^{B} d^{B}}\right)(1-\pi)
\end{array}\right.
\end{aligned}
$$

which for any $\pi \in\left[\frac{5}{7}, 1\right]$ and $a^{* A} \in[0,1]$ is impossible.

\section{Case 2.2}

$$
\begin{aligned}
& \delta^{A}\left(p^{A}\right)=\left\{\begin{array}{lll}
1 & \text { if } & p^{A}=1 \\
0 & \text { if } & p^{A}=2
\end{array} \text { and } \delta^{B}\left(p^{B}\right)=\left\{\begin{array}{lll}
0 & \text { if } p^{B}=1 \\
0 & \text { if } & p^{B}=2
\end{array}\right.\right. \\
& \mu^{A}=\mu\left(p^{A}=1 \mid d^{A}\right)=\left\{\begin{array}{ll}
0 & \text { if } d^{A}=0 \\
1 & \text { if } d^{A}=1
\end{array} \quad \text { and } \mu^{B}=\mu\left(p^{B}=1 \mid d^{B}\right)=\left\{\begin{array}{cc}
\pi & \text { if } d^{B}=0 \\
\pi & \text { if } d^{B}=1
\end{array}\right.\right.
\end{aligned}
$$

- when $\left(d^{A}=0, d^{B}=0\right)$, then

$$
E\left(\frac{p^{A} a}{p^{A}+p^{B}+p^{A} d^{A}}\right)+E\left(\frac{p^{B}(1-a)}{p^{A}+p^{B}+p^{B} d^{B}}\right)=\frac{2 a \pi+3-\pi}{6} \Rightarrow a^{* A}\left(d^{A}=0, d^{B}=0\right)=1
$$


- when $\left(d^{A}=0, d^{B}=1\right)$, then

$$
E\left(\frac{p^{A} a}{p^{A}+p^{B}+p^{A} d^{A}}\right)+E\left(\frac{p^{B}(1-a)}{p^{A}+p^{B}+p^{B} d^{B}}\right)=\frac{3 a \pi+2 a+4-\pi}{12} \Rightarrow a^{* A}\left(d^{A}=0, d^{B}=1\right)=1
$$

- when $\left(d^{A}=1, d^{B}=0\right)$, then

$$
E\left(\frac{p^{A} a}{p^{A}+p^{B}+p^{A} d^{A}}\right)+E\left(\frac{p^{B}(1-a)}{p^{A}+p^{B}+p^{B} d^{B}}\right)=\frac{3 a \pi-5 a-2 \pi+8}{12} \Rightarrow a^{* A}\left(d^{A}=1, d^{B}=0\right)=0
$$

- when $\left(d^{A}=1, d^{B}=1\right)$, then

$$
E\left(\frac{p^{A} a}{p^{A}+p^{B}+p^{A} d^{A}}\right)+E\left(\frac{p^{B}(1-a)}{p^{A}+p^{B}+p^{B} d^{B}}\right)=\frac{9 a \pi-9 a+24-4 \pi}{60} \Rightarrow a^{* A}\left(d^{A}=1, d^{B}=1\right)=0
$$

To conclude

$$
a^{* A}\left(d^{A}, d^{B}\right) \in\left\{\begin{array}{lll}
\{1\} & \text { if } & \left(d^{A}=0, d^{B}=0\right) \\
\{1\} & \text { if } & \left(d^{A}=0, d^{B}=1\right) \\
\{0\} & \text { if } & \left(d^{A}=1, d^{B}=0\right) \\
\{0\} & \text { if } & \left(d^{A}=1, d^{B}=1\right)
\end{array}\right.
$$

First stage: the governments' rational choices

$$
\begin{gathered}
\delta^{A}\left(p^{A}\right)=\left\{\begin{array}{ll}
1 & \text { if } p^{A}=1 \\
0 & \text { if } p^{A}=2
\end{array} \text { and } \delta^{B}\left(p^{B}\right)=\left\{\begin{array}{lll}
0 & \text { if } p^{B}=1 \\
0 & \text { if } p^{B}=2
\end{array}\right.\right. \\
a^{* A}\left(d^{A}, d^{B}\right) \in \begin{cases}\{1\} & \text { if }\left(d^{A}=0, d^{B}=0\right) \\
\{1\} & \text { if }\left(d^{A}=0, d^{B}=1\right) \\
\{0\} & \text { if }\left(d^{A}=1, d^{B}=0\right) \\
\{0\} & \text { if }\left(d^{A}=1, d^{B}=1\right)\end{cases}
\end{gathered}
$$

are part of a PBE if and only if

$$
\begin{aligned}
& \begin{cases}-\left(\gamma d^{* A}+\frac{a^{* A}}{p^{A}+1+p^{A} d^{* A}}\right) \pi-\left(\gamma d^{* A}+\frac{a^{* A}}{p^{A}+2+p^{A} d^{* A}}\right)(1-\pi) \geq & \text { if } p^{A}=1 \\
\geq-\left(\gamma d^{A}+\frac{a^{* A}}{p^{A}+1+p^{A} d^{A}}\right) \pi-\left(\gamma d^{A}+\frac{a^{* A}}{p^{A}+2+p^{A} d^{A}}\right)(1-\pi) & \\
-\left(\gamma d^{* A}+\frac{a^{* A}}{p^{A}+1+p^{A} d^{* A}}\right) \pi-\left(\gamma d^{* A}+\frac{a^{* A}}{p^{A}+2+p^{A} d^{* A}}\right)(1-\pi) \geq & \text { if } p^{A}=2 \\
\geq-\left(\gamma d^{A}+\frac{a^{* A}}{p^{A}+1+p^{A} d^{A}}\right) \pi-\left(\gamma d^{A}+\frac{a^{* A}}{p^{A}+2+p^{A} d^{A}}\right)(1-\pi) & \end{cases} \\
& \left\{\begin{array}{l}
-\left(\gamma d^{* B}+\frac{1-a^{* A}}{p^{B}+1+p^{B} d^{* B}}\right) \pi-\left(\gamma d^{* B}+\frac{1-a^{* A}}{p^{B}+2+p^{B} d^{* B}}\right)(1-\pi) \geq \\
\geq-\left(\gamma d^{B}+\frac{1-a^{* A}}{p^{B}+1+p^{B} d^{B}}\right) \pi-\left(\gamma d^{B}+\frac{1-a^{* A}}{p^{B}+2+p^{B} d^{B}}\right)(1-\pi) \\
-\left(\gamma d^{* B}+\frac{1-a^{* A}}{p^{B}+1+p^{B} d^{* B}}\right) \pi-\left(\gamma d^{* B}+\frac{1-a^{* A}}{p^{B}+2+p^{B} d^{* B}}\right)(1-\pi) \geq \\
\geq-\left(\gamma d^{B}+\frac{1-a^{* A}}{p^{B}+1+p^{B} d^{B}}\right) \pi-\left(\gamma d^{B}+\frac{1-a^{* A}}{p^{B}+2+p^{B} d^{B}}\right)(1-\pi)
\end{array}\right.
\end{aligned}
$$

which are possible when

$$
\gamma \in\left[\left(\frac{7}{12}\right) \pi ;\left(\frac{5}{6}\right) \pi\right]
$$




\section{Case 2.3}

$$
\begin{gathered}
\delta^{A}\left(p^{A}\right)=\left\{\begin{array}{ll}
0 & \text { if } p^{A}=1 \\
1 & \text { if } p^{A}=2
\end{array} \text { and } \delta^{B}\left(p^{B}\right)= \begin{cases}1 & \text { if } p^{B}=1 \\
1 & \text { if } p^{B}=2\end{cases} \right. \\
\mu^{A}=\mu\left(p^{A}=1 \mid d^{A}\right)=\left\{\begin{array}{ll}
1 & \text { if } d^{A}=0 \\
0 & \text { if } d^{A}=1
\end{array} \text { and } \mu^{B}=\mu\left(p^{B}=1 \mid d^{B}\right)= \begin{cases}\pi & \text { if } d^{B}=0 \\
\pi & \text { if } d^{B}=1\end{cases} \right.
\end{gathered}
$$

- when $\left(d^{A}=0, d^{B}=0\right)$, then

$$
E\left(\frac{p^{A} a}{p^{A}+p^{B}+p^{A} d^{A}}\right)+E\left(\frac{p^{B}(1-a)}{p^{A}+p^{B}+p^{B} d^{B}}\right)=\frac{-2 a+2 a \pi+4-\pi}{6} \Rightarrow a^{* A}\left(d^{A}=0, d^{B}=0\right)=0
$$

- when $\left(d^{A}=0, d^{B}=1\right)$, then

$$
E\left(\frac{p^{A} a}{p^{A}+p^{B}+p^{A} d^{A}}\right)+E\left(\frac{p^{B}(1-a)}{p^{A}+p^{B}+p^{B} d^{B}}\right)=\frac{(7 \pi-2) a+12-2 \pi}{30} \Rightarrow a^{* A}\left(d^{A}=0, d^{B}=1\right)=\left\{\begin{array}{l}
0 \\
1
\end{array}\right.
$$

- when $\left(d^{A}=1, d^{B}=0\right)$, then

$$
E\left(\frac{p^{A} a}{p^{A}+p^{B}+p^{A} d^{A}}\right)+E\left(\frac{p^{B}(1-a)}{p^{A}+p^{B}+p^{B} d^{B}}\right)=\frac{(7 \pi-5) a+15-5 \pi}{30} \Rightarrow a^{* A}\left(d^{A}=1, d^{B}=0\right)=\left\{\begin{array}{l}
0 \\
1
\end{array}\right.
$$

- when $\left(d^{A}=1, d^{B}=1\right)$, then

$$
E\left(\frac{p^{A} a}{p^{A}+p^{B}+p^{A} d^{A}}\right)+E\left(\frac{p^{B}(1-a)}{p^{A}+p^{B}+p^{B} d^{B}}\right)=\frac{9 a \pi-5 \pi+20}{60} \Rightarrow a^{* A}\left(d^{A}=1, d^{B}=1\right)=1
$$

To conclude

- if $\pi \in\left[0, \frac{2}{7}\right]$

$$
a^{* A}\left(d^{A}, d^{B}\right) \in\left\{\begin{array}{lll}
\{0\} & \text { if } & \left(d^{A}=0, d^{B}=0\right) \\
\{0\} & \text { if } \quad\left(d^{A}=0, d^{B}=1\right) \\
\{0\} & \text { if } \left.\quad d^{A}=1, d^{B}=0\right) \\
\{1\} & \text { if } \quad\left(d^{A}=1, d^{B}=1\right)
\end{array}\right.
$$

- if $\pi \in\left[\frac{2}{7}, \frac{5}{7}\right]$

$$
a^{* A}\left(d^{A}, d^{B}\right) \in\left\{\begin{array}{lll}
\{0\} & \text { if } & \left(d^{A}=0, d^{B}=0\right) \\
\{1\} & \text { if } & \left(d^{A}=0, d^{B}=1\right) \\
\{0\} & \text { if } & \left(d^{A}=1, d^{B}=0\right) \\
\{1\} & \text { if } & \left(d^{A}=1, d^{B}=1\right)
\end{array}\right.
$$

- if $\pi \in\left[\frac{5}{7}, 1\right]$

$$
a^{* A}\left(d^{A}, d^{B}\right) \in\left\{\begin{array}{lll}
\{0\} & \text { if } & \left(d^{A}=0, d^{B}=0\right) \\
\{1\} & \text { if } & \left(d^{A}=0, d^{B}=1\right) \\
\{1\} & \text { if } & \left(d^{A}=1, d^{B}=0\right) \\
\{1\} & \text { if } & \left(d^{A}=1, d^{B}=1\right)
\end{array}\right.
$$


First stage: the governments' rational choices

Case $\pi \in\left[0, \frac{2}{7}\right]$

$$
\begin{gathered}
\delta^{A}\left(p^{A}\right)=\left\{\begin{array}{ll}
0 & \text { if } p^{A}=1 \\
1 & \text { if } p^{A}=2
\end{array} \text { and } \delta^{B}\left(p^{B}\right)=\left\{\begin{array}{lll}
1 & \text { if } p^{B}=1 \\
1 & \text { if } p^{B}=2
\end{array}\right.\right. \\
a^{* A}\left(d^{A}, d^{B}\right) \in \begin{cases}\{0\} & \text { if } \quad\left(d^{A}=0, d^{B}=0\right) \\
\{0\} & \text { if } \quad\left(d^{A}=0, d^{B}=1\right) \\
\{0\} & \text { if } \quad\left(d^{A}=1, d^{B}=0\right) \\
\{1\} & \text { if }\left(d^{A}=1, d^{B}=1\right)\end{cases}
\end{gathered}
$$

are part of a PBE if and only if

$$
\begin{aligned}
& \left\{\begin{array}{l}
-\left(\gamma d^{* A}+\frac{a^{* A}}{p^{A}+1+p^{A} d^{* A}}\right) \pi-\left(\gamma d^{* A}+\frac{a^{* A}}{p^{A}+2+p^{A} d^{* A}}\right)(1-\pi) \geq \\
\geq-\left(\gamma d^{A}+\frac{a^{* A}}{p^{A}+1+p^{A} d^{A}}\right) \pi-\left(\gamma d^{A}+\frac{a^{* A}}{p^{A}+2+p^{A} d^{A}}\right)(1-\pi) \\
-\left(\gamma d^{* A}+\frac{a^{* A}}{p^{A}+1+p^{A} d^{* A}}\right) \pi-\left(\gamma d^{* A}+\frac{a^{* A}}{p^{A}+2+p^{A} d^{* A}}\right)(1-\pi) \geq \\
\geq-\left(\gamma d^{A}+\frac{a^{* A}}{p^{A}+1+p^{A} d^{A}}\right) \pi-\left(\gamma d^{A}+\frac{a^{* A}}{p^{A}+2+p^{A} d^{A}}\right)(1-\pi)
\end{array}\right. \\
& \left\{\begin{array}{l}
-\left(\gamma d^{* B}+\frac{1-a^{* A}}{p^{B}+1+p^{B} d^{* B}}\right) \pi-\left(\gamma d^{* B}+\frac{1-a^{* A}}{p^{B}+2+p^{B} d^{* B}}\right)(1-\pi) \geq \\
\geq-\left(\gamma d^{B}+\frac{1-a^{* A}}{p^{B}+1+p^{B} d^{B}}\right) \pi-\left(\gamma d^{B}+\frac{1-a^{* A}}{p^{B}+2+p^{B} d^{B}}\right)(1-\pi) \\
-\left(\gamma d^{* B}+\frac{1-a^{* A}}{p^{B}+1+p^{B} d^{* B}}\right) \pi-\left(\gamma d^{* B}+\frac{1-a^{* A}}{p^{B}+2+p^{B} d^{* B}}\right)(1-\pi) \geq \\
\geq-\left(\gamma d^{B}+\frac{1-a^{* A}}{p^{B}+1+p^{B} d^{B}}\right) \pi-\left(\gamma d^{B}+\frac{1-a^{* A}}{p^{B}+2+p^{B} d^{B}}\right)(1-\pi)
\end{array}\right.
\end{aligned}
$$

which for any $\pi \in\left[0, \frac{2}{7}\right]$ and $a^{* A} \in[0,1]$ is impossible.

Case $\pi \in\left[\frac{2}{7}, \frac{5}{7}\right]$

$$
\begin{gathered}
\delta^{A}\left(p^{A}\right)=\left\{\begin{array}{ll}
0 & \text { if } p^{A}=1 \\
1 & \text { if } p^{A}=2
\end{array} \text { and } \delta^{B}\left(p^{B}\right)=\left\{\begin{array}{lll}
1 & \text { if } p^{B}=1 \\
1 & \text { if } p^{B}=2
\end{array}\right.\right. \\
a^{* A}\left(d^{A}, d^{B}\right) \in \begin{cases}\{0\} & \text { if } \quad\left(d^{A}=0, d^{B}=0\right) \\
\{1\} & \text { if }\left(d^{A}=0, d^{B}=1\right) \\
\{0\} & \text { if } \quad\left(d^{A}=1, d^{B}=0\right) \\
\{1\} & \text { if }\left(d^{A}=1, d^{B}=1\right)\end{cases}
\end{gathered}
$$

are part of a PBE if and only

$$
\left\{\begin{array}{l}
-\left(\gamma d^{* A}+\frac{a^{* A}}{p^{A}+1+p^{A} d^{* A}}\right) \pi-\left(\gamma d^{* A}+\frac{a^{* A}}{p^{A}+2+p^{A} d^{* A}}\right)(1-\pi) \geq \\
\geq-\left(\gamma d^{A}+\frac{a^{* A}}{p^{A}+1+p^{A} d^{A}}\right) \pi-\left(\gamma d^{A}+\frac{a^{* A}}{p^{A}+2+p^{A} d^{A}}\right)(1-\pi) \\
-\left(\gamma d^{* A}+\frac{a^{* A}}{p^{A}+1+p^{A} d^{* A}}\right) \pi-\left(\gamma d^{* A}+\frac{a^{* A}}{p^{A}+2+p^{A} d^{* A}}\right)(1-\pi) \geq \\
\geq-\left(\gamma d^{A}+\frac{a^{* A}}{p^{A}+1+p^{A} d^{A}}\right) \pi-\left(\gamma d^{A}+\frac{a^{* A}}{p^{A}+2+p^{A} d^{A}}\right)(1-\pi)
\end{array}\right.
$$




$$
\left\{\begin{array}{l}
-\left(\gamma d^{* B}+\frac{1-a^{* A}}{p^{B}+1+p^{B} d^{* B}}\right) \pi-\left(\gamma d^{* B}+\frac{1-a^{* A}}{p^{B}+2+p^{B} d^{* B}}\right)(1-\pi) \geq \\
\geq-\left(\gamma d^{B}+\frac{1-a^{* A}}{p^{B}+1+p^{B} d^{B}}\right) \pi-\left(\gamma d^{B}+\frac{1-a^{* A}}{p^{B}+2+p^{B} d^{B}}\right)(1-\pi) \\
-\left(\gamma d^{* B}+\frac{1-a^{* A}}{p^{B}+1+p^{B} d^{* B}}\right) \pi-\left(\gamma d^{* B}+\frac{1-a^{* A}}{p^{B}+2+p^{B} d^{* B}}\right)(1-\pi) \geq \\
\geq-\left(\gamma d^{B}+\frac{1-a^{* A}}{p^{B}+1+p^{B} d^{B}}\right) \pi-\left(\gamma d^{B}+\frac{1-a^{* A}}{p^{B}+2+p^{B} d^{B}}\right)(1-\pi)
\end{array}\right.
$$

which for any $\pi \in\left[\frac{2}{7}, \frac{5}{7}\right]$ and $a^{* A} \in[0,1]$ is impossible.

Case $\pi \in\left[\frac{5}{7}, 1\right]$

$$
\begin{gathered}
\delta^{A}\left(p^{A}\right)=\left\{\begin{array}{ll}
0 & \text { if } p^{A}=1 \\
1 & \text { if } p^{A}=2
\end{array} \text { and } \delta^{B}\left(p^{B}\right)=\left\{\begin{array}{lll}
1 & \text { if } p^{B}=1 \\
1 & \text { if } p^{B}=2
\end{array}\right.\right. \\
a^{* A}\left(d^{A}, d^{B}\right) \in \begin{cases}\{0\} & \text { if } \quad\left(d^{A}=0, d^{B}=0\right) \\
\{1\} & \text { if }\left(d^{A}=0, d^{B}=1\right) \\
\{1\} & \text { if } \quad\left(d^{A}=1, d^{B}=0\right) \\
\{1\} & \text { if }\left(d^{A}=1, d^{B}=1\right)\end{cases}
\end{gathered}
$$

are part of a PBE if and only if

$$
\begin{aligned}
& \left\{\begin{array}{l}
-\left(\gamma d^{* A}+\frac{a^{* A}}{p^{A}+1+p^{A} d^{* A}}\right) \pi-\left(\gamma d^{* A}+\frac{a^{* A}}{p^{A}+2+p^{A} d^{* A}}\right)(1-\pi) \geq \\
\geq-\left(\gamma d^{A}+\frac{a^{* A}}{p^{A}+1+p^{A} d^{A}}\right) \pi-\left(\gamma d^{A}+\frac{a^{* A}}{p^{A}+2+p^{A} d^{A}}\right)(1-\pi) \\
-\left(\gamma d^{* A}+\frac{a^{* A}}{p^{A}+1+p^{A} d^{* A}}\right) \pi-\left(\gamma d^{* A}+\frac{a^{* A}}{p^{A}+2+p^{A} d^{* A}}\right)(1-\pi) \geq \\
\geq-\left(\gamma d^{A}+\frac{a^{* A}}{p^{A}+1+p^{A} d^{A}}\right) \pi-\left(\gamma d^{A}+\frac{a^{* A}}{p^{A}+2+p^{A} d^{A}}\right)(1-\pi)
\end{array}\right. \\
& \left\{\begin{array}{l}
-\left(\gamma d^{* B}+\frac{1-a^{* A}}{p^{B}+1+p^{B} d^{* B}}\right) \pi-\left(\gamma d^{* B}+\frac{1-a^{* A}}{p^{B}+2+p^{B} d^{* B}}\right)(1-\pi) \geq \\
\geq-\left(\gamma d^{B}+\frac{1-a^{* A}}{p^{B}+1+p^{B} d^{B}}\right) \pi-\left(\gamma d^{B}+\frac{1-a^{* A}}{p^{B}+2+p^{B} d^{B}}\right)(1-\pi) \\
-\left(\gamma d^{* B}+\frac{1-a^{* A}}{p^{B}+1+p^{B} d^{* B}}\right) \pi-\left(\gamma d^{* B}+\frac{1-a^{* A}}{p^{B}+2+p^{B} d^{* B}}\right)(1-\pi) \geq \\
\geq-\left(\gamma d^{B}+\frac{1-a^{* A}}{p^{B}+1+p^{B} d^{B}}\right) \pi-\left(\gamma d^{B}+\frac{1-a^{*}}{p^{B}+2+p^{B} d^{B}}\right)(1-\pi)
\end{array}\right.
\end{aligned}
$$

which for any $\pi \in\left[\frac{5}{7}, 1\right]$ and $a^{* A} \in[0,1]$ is impossible.

\section{Case 2.4}

$$
\begin{aligned}
& \delta^{A}\left(p^{A}\right)=\left\{\begin{array}{ll}
1 & \text { if } p^{A}=1 \\
0 & \text { if } p^{A}=2
\end{array} \text { and } \delta^{B}\left(p^{B}\right)= \begin{cases}1 & \text { if } p^{B}=1 \\
1 & \text { if } p^{B}=2\end{cases} \right. \\
& \mu^{A}=\mu\left(p^{A}=1 \mid d^{A}\right)=\left\{\begin{array}{ll}
0 & \text { if } d^{A}=0 \\
1 & \text { if } d^{A}=1
\end{array} \quad \text { and } \mu^{B}=\mu\left(p^{B}=1 \mid d^{B}\right)=\left\{\begin{array}{cc}
\pi & \text { if } d^{B}=0 \\
\pi & \text { if } d^{B}=1
\end{array}\right.\right.
\end{aligned}
$$

- when $\left(d^{A}=0, d^{B}=0\right)$, then

$$
E\left(\frac{p^{A} a}{p^{A}+p^{B}+p^{A} d^{A}}\right)+E\left(\frac{p^{B}(1-a)}{p^{A}+p^{B}+p^{B} d^{B}}\right)=\frac{2 a \pi+3-\pi}{6} \Rightarrow a^{* A}\left(d^{A}=0, d^{B}=0\right)=1
$$


- when $\left(d^{A}=0, d^{B}=1\right)$, then

$$
E\left(\frac{p^{A} a}{p^{A}+p^{B}+p^{A} d^{A}}\right)+E\left(\frac{p^{B}(1-a)}{p^{A}+p^{B}+p^{B} d^{B}}\right)=\frac{3 a \pi+2 a+4-\pi}{12} \Rightarrow a^{* A}\left(d^{A}=0, d^{B}=1\right)=1
$$

- when $\left(d^{A}=1, d^{B}=0\right)$, then

$$
E\left(\frac{p^{A} a}{p^{A}+p^{B}+p^{A} d^{A}}\right)+E\left(\frac{p^{B}(1-a)}{p^{A}+p^{B}+p^{B} d^{B}}\right)=\frac{3 a \pi-5 a+8-2 \pi}{12} \Rightarrow a^{* A}\left(d^{A}=1, d^{B}=0\right)=0
$$

- when $\left(d^{A}=1, d^{B}=1\right)$, then

$$
E\left(\frac{p^{A} a}{p^{A}+p^{B}+p^{A} d^{A}}\right)+E\left(\frac{p^{B}(1-a)}{p^{A}+p^{B}+p^{B} d^{B}}\right)=\frac{9 a \pi-9 a+24-4 \pi}{60} \Rightarrow a^{* A}\left(d^{A}=1, d^{B}=1\right)=0
$$

To conclude

$$
a^{* A}\left(d^{A}, d^{B}\right) \in\left\{\begin{array}{lll}
\{1\} & \text { if } & \left(d^{A}=0, d^{B}=0\right) \\
\{1\} & \text { if } & \left(d^{A}=0, d^{B}=1\right) \\
\{0\} & \text { if } \quad\left(d^{A}=1, d^{B}=0\right) \\
\{0\} & \text { if } \quad\left(d^{A}=1, d^{B}=1\right)
\end{array}\right.
$$

First stage: the governments' rational choices

$$
\begin{gathered}
\delta^{A}\left(p^{A}\right)=\left\{\begin{array}{ll}
1 & \text { if } p^{A}=1 \\
0 & \text { if } p^{A}=2
\end{array} \text { and } \delta^{B}\left(p^{B}\right)=\left\{\begin{array}{lll}
1 & \text { if } p^{B}=1 \\
1 & \text { if } p^{B}=2
\end{array}\right.\right. \\
a^{* A}\left(d^{A}, d^{B}\right) \in \begin{cases}\{1\} & \text { if } \quad\left(d^{A}=0, d^{B}=0\right) \\
\{1\} & \text { if }\left(d^{A}=0, d^{B}=1\right) \\
\{0\} & \text { if }\left(d^{A}=1, d^{B}=0\right) \\
\{0\} & \text { if }\left(d^{A}=1, d^{B}=1\right)\end{cases}
\end{gathered}
$$

are part of a PBE if and only if

$$
\begin{aligned}
& \left\{\begin{array}{c}
-\left(\gamma d^{* A}+\frac{a^{* A}}{p^{A}+1+p^{A} d^{* A}}\right) \pi-\left(\gamma d^{* A}+\frac{a^{* A}}{p^{A}+2+p^{A} d^{* A}}\right)(1-\pi) \geq \\
\geq-\left(\gamma d^{A}+\frac{a^{* A}}{p^{A}+1+p^{A} d^{A}}\right) \pi-\left(\gamma d^{A}+\frac{a^{* A}}{p^{A}+2+p^{A} d^{A}}\right)(1-\pi) \\
-\left(\gamma d^{* A}+\frac{a^{* A}}{p^{A}+1+p^{A} d^{* A}}\right) \pi-\left(\gamma d^{* A}+\frac{a^{* A}}{p^{A}+2+p^{A} d^{* A}}\right)(1-\pi) \geq \quad \text { if } p^{A}=1 \\
\geq-\left(\gamma d^{A}+\frac{a^{* A}}{p^{A}+1+p^{A} d^{A}}\right) \pi-\left(\gamma d^{A}+\frac{a^{* A}}{p^{A}+2+p^{A} d^{A}}\right)(1-\pi)
\end{array}\right. \\
& \left\{\begin{array}{c}
-\left(\gamma d^{* B}+\frac{1-a^{* A}}{p^{B}+1+p^{B} d^{* B}}\right) \pi-\left(\gamma d^{* B}+\frac{1-a^{* A}}{p^{B}+2+p^{B} d^{* B}}\right)(1-\pi) \geq \quad \text { if } p^{B}=1 \\
\geq-\left(\gamma d^{B}+\frac{1-a^{* A}}{p^{B}+1+p^{B} d^{B}}\right) \pi-\left(\gamma d^{B}+\frac{1-a^{* A}}{p^{B}+2+p^{B} d^{B}}\right)(1-\pi) \\
-\left(\gamma d^{* B}+\frac{1-a^{* A}}{p^{B}+1+p^{B} d^{* B}}\right) \pi-\left(\gamma d^{* B}+\frac{1-a^{* A}}{p^{B}+2+p^{B} d^{* B}}\right)(1-\pi) \geq \quad \text { if } p^{B}=2 \\
\geq-\left(\gamma d^{B}+\frac{1-a^{* A}}{p^{B}+1+p^{B} d^{B}}\right) \pi-\left(\gamma d^{B}+\frac{1-a^{* A}}{p^{B}+2+p^{B} d^{B}}\right)(1-\pi)
\end{array}\right.
\end{aligned}
$$

which are impossible. 
3. A pooling and B separating Being the game symmetric, we get exactly the same conclusion of the previous paragraph.

\section{A and B pooling}

\section{Case 4.1}

$$
\begin{gathered}
\delta^{A}\left(p^{A}\right)=\left\{\begin{array}{ll}
0 & \text { if } p^{A}=1 \\
0 & \text { if } p^{A}=2
\end{array} \text { and } \delta^{B}\left(p^{B}\right)= \begin{cases}0 & \text { if } p^{B}=1 \\
0 & \text { if } p^{B}=2\end{cases} \right. \\
\mu^{j}=\mu\left(p^{j}=1 \mid d^{j}\right)= \begin{cases}\pi & \text { if } d^{j}=0 \\
\pi & \text { if } d^{j}=1 .\end{cases}
\end{gathered}
$$

- when $\left(d^{A}=0, d^{B}=0\right)$, then

$$
\begin{gathered}
E\left(\frac{p^{A} a}{p^{A}+p^{B}+p^{A} d^{A}}\right)+E\left(\frac{p^{B}(1-a)}{p^{A}+p^{B}+p^{B} d^{B}}\right)= \\
=\frac{a \pi^{2}}{2}+\frac{a \pi(1-\pi)}{3}+\frac{2 a(1-\pi) \pi}{3}+\frac{a(1-\pi)^{2}}{2}+ \\
+\frac{(1-a) \pi^{2}}{2}+\frac{2(1-a) \pi(1-\pi)}{3}+\frac{(1-a)(1-\pi) \pi}{3}+\frac{(1-a)(1-\pi)^{2}}{2} \Rightarrow \\
\Rightarrow a^{* A}\left(d^{A}=0, d^{B}=0\right) \in[0,1]
\end{gathered}
$$

- when $\left(d^{A}=0, d^{B}=1\right)$, then

$$
\begin{gathered}
E\left(\frac{p^{A} a}{p^{A}+p^{B}+p^{A} d^{A}}\right)+E\left(\frac{p^{B}(1-a)}{p^{A}+p^{B}+p^{B} d^{B}}\right)= \\
=\frac{a \pi^{2}}{2}+\frac{a \pi(1-\pi)}{3}+\frac{2 a(1-\pi) \pi}{3}+\frac{a(1-\pi)^{2}}{2}+ \\
+\frac{(1-a) \pi^{2}}{3}+\frac{2(1-a) \pi(1-\pi)}{5}+\frac{(1-a)(1-\pi) \pi}{4}+\frac{(1-a)(1-\pi)^{2}}{3} \Rightarrow \\
\Rightarrow a^{* A}\left(d^{A}=0, d^{B}=1\right)=1
\end{gathered}
$$

- when $\left(d^{A}=1, d^{B}=0\right)$, then

$$
\begin{gathered}
E\left(\frac{p^{A} a}{p^{A}+p^{B}+p^{A} d^{A}}\right)+E\left(\frac{p^{B}(1-a)}{p^{A}+p^{B}+p^{B} d^{B}}\right)= \\
=\frac{a \pi^{2}}{3}+\frac{a \pi(1-\pi)}{4}+\frac{2 a(1-\pi) \pi}{5}+\frac{a(1-\pi)^{2}}{3}+ \\
+\frac{(1-a) \pi^{2}}{2}+\frac{2(1-a) \pi(1-\pi)}{3}+\frac{(1-a)(1-\pi) \pi}{3}+\frac{(1-a)(1-\pi)^{2}}{2} \Rightarrow \\
\Rightarrow a^{* A}\left(d^{A}=1, d^{B}=0\right)=0
\end{gathered}
$$


- when $\left(d^{A}=1, d^{B}=1\right)$, then

$$
\begin{gathered}
E\left(\frac{p^{A} a}{p^{A}+p^{B}+p^{A} d^{A}}\right)+E\left(\frac{p^{B}(1-a)}{p^{A}+p^{B}+p^{B} d^{B}}\right)= \\
=\frac{a \pi^{2}}{3}+\frac{a \pi(1-\pi)}{4}+\frac{2 a(1-\pi) \pi}{5}+\frac{a(1-\pi)^{2}}{3}+ \\
+\frac{(1-a) \pi^{2}}{3}+\frac{2(1-a) \pi(1-\pi)}{5}+\frac{(1-a)(1-\pi) \pi}{4}+\frac{(1-a)(1-\pi)^{2}}{3} \Rightarrow \\
\Rightarrow a^{* A}\left(d^{A}=1, d^{B}=0\right) \in[0,1]
\end{gathered}
$$

To conclude

$$
a^{* A}\left(d^{A}, d^{B}\right) \in\left\{\begin{array}{ccc}
{[0,1]} & \text { if } & \left(d^{A}=0, d^{B}=0\right) \\
\{1\} & \text { if } & \left(d^{A}=0, d^{B}=1\right) \\
\{0\} & \text { if } & \left(d^{A}=1, d^{B}=0\right) \\
{[0,1]} & \text { if } & \left(d^{A}=1, d^{B}=1\right)
\end{array}\right.
$$

First stage: the governments' rational choices

$$
\begin{gathered}
\delta^{A}\left(p^{A}\right)=\left\{\begin{array}{ll}
0 & \text { if } p^{A}=1 \\
0 & \text { if } p^{A}=2
\end{array} \text { and } \delta^{B}\left(p^{B}\right)=\left\{\begin{array}{lll}
0 & \text { if } p^{B}=1 \\
0 & \text { if } p^{B}=2
\end{array}\right.\right. \\
a^{* A}\left(d^{A}, d^{B}\right) \in\left\{\begin{array}{cc}
{[0,1]} & \text { if }\left(d^{A}=0, d^{B}=0\right) \\
\{1\} & \text { if }\left(d^{A}=0, d^{B}=1\right) \\
\{0\} & \text { if }\left(d^{A}=1, d^{B}=0\right) \\
{[0,1]} & \text { if }\left(d^{A}=1, d^{B}=1\right)
\end{array}\right.
\end{gathered}
$$

are part of a PBE if and only if

$$
\begin{aligned}
& \begin{cases}-\left(\gamma d^{* A}+\frac{a^{* A}}{p^{A}+1+p^{A} d^{* A}}\right) \pi-\left(\gamma d^{* A}+\frac{a^{* A}}{p^{A}+2+p^{A} d^{* A}}\right)(1-\pi) \geq & \text { if } p^{A}=1 \\
\geq-\left(\gamma d^{A}+\frac{a^{* A}}{p^{A}+1+p^{A} d^{A}}\right) \pi-\left(\gamma d^{A}+\frac{a^{* A}}{p^{A}+2+p^{A} d^{A}}\right)(1-\pi) & \\
-\left(\gamma d^{* A}+\frac{a^{* A}}{p^{A}+1+p^{A} d^{* A}}\right) \pi-\left(\gamma d^{* A}+\frac{a^{* A}}{p^{A}+2+p^{A} d^{* A}}\right)(1-\pi) \geq & \text { if } p^{A}=2 \\
\geq-\left(\gamma d^{A}+\frac{a^{* A}}{p^{A}+1+p^{A} d^{A}}\right) \pi-\left(\gamma d^{A}+\frac{a^{* A}}{p^{A}+2+p^{A} d^{A}}\right)(1-\pi) & \end{cases} \\
& \left\{\begin{array}{l}
-\left(\gamma d^{* B}+\frac{1-a^{* A}}{p^{B}+1+p^{B} d^{* B}}\right) \pi-\left(\gamma d^{* B}+\frac{1-a^{* A}}{p^{B}+2+p^{B} d^{* B}}\right)(1-\pi) \geq \\
\geq-\left(\gamma d^{B}+\frac{1-a^{* A}}{p^{B}+1+p^{B} d^{B}}\right) \pi-\left(\gamma d^{B}+\frac{1-a^{* A}}{p^{B}+2+p^{B} d^{B}}\right)(1-\pi) \\
-\left(\gamma d^{* B}+\frac{1-a^{* A}}{p^{B}+1+p^{B} d^{* B}}\right) \pi-\left(\gamma d^{* B}+\frac{1-a^{* A}}{p^{B}+2+p^{B} d^{* B}}\right)(1-\pi) \geq \\
\geq-\left(\gamma d^{B}+\frac{1-a^{* A}}{p^{B}+1+p^{B} d^{B}}\right) \pi-\left(\gamma d^{B}+\frac{1-a^{* A}}{p^{B}+2+p^{B} d^{B}}\right)(1-\pi)
\end{array}\right.
\end{aligned}
$$

which are possible when

$\gamma \in\left[\left(\frac{a^{* A}}{2}\right) \pi+\left(\frac{a^{* A}}{3}\right)(1-\pi), 1\right] \cap\left[\left(\frac{1-a^{* A}}{2}\right) \pi+\left(\frac{1-a^{* A}}{3}\right)(1-\pi), 1\right]$. 


\section{Case 4.2}

$$
\begin{gathered}
\delta^{A}\left(p^{A}\right)=\left\{\begin{array}{ll}
0 & \text { if } p^{A}=1 \\
0 & \text { if } p^{A}=2
\end{array} \text { and } \delta^{B}\left(p^{B}\right)=\left\{\begin{array}{lll}
1 & \text { if } p^{B}=1 \\
1 & \text { if } p^{B}=2
\end{array}\right.\right. \\
\mu^{A}=\mu\left(p^{A}=1 \mid d^{A}\right)=\left\{\begin{array}{ll}
\pi & \text { if } d^{A}=0 \\
\pi & \text { if } d^{A}=1
\end{array} \text { and } \mu^{B}=\mu\left(p^{B}=1 \mid d^{B}\right)= \begin{cases}\pi & \text { if } d^{B}=0 \\
\pi & \text { if } d^{B}=1\end{cases} \right.
\end{gathered}
$$

- when $\left(d^{A}=0, d^{B}=0\right)$, then

$$
\begin{gathered}
E\left(\frac{p^{A} a}{p^{A}+p^{B}+p^{A} d^{A}}\right)+E\left(\frac{p^{B}(1-a)}{p^{A}+p^{B}+p^{B} d^{B}}\right)= \\
=\frac{a \pi^{2}}{2}+\frac{a \pi(1-\pi)}{3}+\frac{2 a(1-\pi) \pi}{3}+\frac{a(1-\pi)^{2}}{2}+ \\
+\frac{(1-a) \pi^{2}}{2}+\frac{2(1-a) \pi(1-\pi)}{3}+\frac{(1-a)(1-\pi) \pi}{3}+\frac{(1-a)(1-\pi)^{2}}{2} \Rightarrow \\
\Rightarrow a^{* A}\left(d^{A}=0, d^{B}=0\right) \in[0,1]
\end{gathered}
$$

- when $\left(d^{A}=0, d^{B}=1\right)$, then

$$
\begin{gathered}
E\left(\frac{p^{A} a}{p^{A}+p^{B}+p^{A} d^{A}}\right)+E\left(\frac{p^{B}(1-a)}{p^{A}+p^{B}+p^{B} d^{B}}\right)= \\
=\frac{a \pi^{2}}{2}+\frac{a \pi(1-\pi)}{3}+\frac{2 a(1-\pi) \pi}{3}+\frac{a(1-\pi)^{2}}{2}+ \\
+\frac{(1-a) \pi^{2}}{3}+\frac{2(1-a) \pi(1-\pi)}{5}+\frac{(1-a)(1-\pi) \pi}{4}+\frac{(1-a)(1-\pi)^{2}}{3} \Rightarrow \\
\Rightarrow a^{* A}\left(d^{A}=0, d^{B}=1\right)=1
\end{gathered}
$$

- when $\left(d^{A}=1, d^{B}=0\right)$, then

$$
\begin{gathered}
E\left(\frac{p^{A} a}{p^{A}+p^{B}+p^{A} d^{A}}\right)+E\left(\frac{p^{B}(1-a)}{p^{A}+p^{B}+p^{B} d^{B}}\right)= \\
=\frac{a \pi^{2}}{3}+\frac{a \pi(1-\pi)}{4}+\frac{2 a(1-\pi) \pi}{5}+\frac{a(1-\pi)^{2}}{3}+ \\
+\frac{(1-a) \pi^{2}}{2}+\frac{2(1-a) \pi(1-\pi)}{3}+\frac{(1-a)(1-\pi) \pi}{3}+\frac{(1-a)(1-\pi)^{2}}{2} \Rightarrow \\
\Rightarrow a^{* A}\left(d^{A}=0, d^{B}=1\right)=0
\end{gathered}
$$


- when $\left(d^{A}=1, d^{B}=1\right)$, then

$$
\begin{gathered}
E\left(\frac{p^{A} a}{p^{A}+p^{B}+p^{A} d^{A}}\right)+E\left(\frac{p^{B}(1-a)}{p^{A}+p^{B}+p^{B} d^{B}}\right)= \\
=\frac{a \pi^{2}}{3}+\frac{a \pi(1-\pi)}{4}+\frac{2 a(1-\pi) \pi}{5}+\frac{a(1-\pi)^{2}}{3}+ \\
+\frac{(1-a) \pi^{2}}{3}+\frac{2(1-a) \pi(1-\pi)}{5}+\frac{(1-a)(1-\pi) \pi}{4}+\frac{(1-a)(1-\pi)^{2}}{3} \Rightarrow \\
\Rightarrow a^{* A}\left(d^{A}=1, d^{B}=1\right) \in[0,1]
\end{gathered}
$$

To conclude

$$
a^{* j}\left(d^{A}, d^{B}\right) \in\left\{\begin{array}{ccc}
{[0,1]} & \text { if } & \left(d^{A}=0, d^{B}=0\right) \\
\{1\} & \text { if } & \left(d^{A}=0, d^{B}=1\right) \\
\{0\} & \text { if } & \left(d^{A}=1, d^{B}=0\right) \\
{[0,1]} & \text { if } & \left(d^{A}=1, d^{B}=1\right)
\end{array}\right.
$$

First stage: the governments' rational choices

$$
\begin{gathered}
\delta^{A}\left(p^{A}\right)=\left\{\begin{array}{ll}
0 & \text { if } p^{A}=1 \\
0 & \text { if } p^{A}=2
\end{array} \text { and } \delta^{B}\left(p^{B}\right)=\left\{\begin{array}{lll}
1 & \text { if } p^{B}=1 \\
1 & \text { if } p^{B}=2
\end{array}\right.\right. \\
a^{* j}\left(d^{A}, d^{B}\right) \in\left\{\begin{array}{cc}
{[0,1]} & \text { if } \quad\left(d^{A}=0, d^{B}=0\right) \\
\{1\} & \text { if } \quad\left(d^{A}=0, d^{B}=1\right) \\
\{0\} & \text { if } \quad\left(d^{A}=1, d^{B}=0\right) \\
{[0,1]} & \text { if } \quad\left(d^{A}=1, d^{B}=1\right)
\end{array}\right.
\end{gathered}
$$

are part of a PBE if and only if

$$
\begin{aligned}
& \begin{cases}-\left(\gamma d^{* A}+\frac{a^{* A}}{p^{A}+1+p^{A} d^{* A}}\right) \pi-\left(\gamma d^{* A}+\frac{a^{* A}}{p^{A}+2+p^{A} d^{* A}}\right)(1-\pi) \geq & \text { if } p^{A}=1 \\
\geq-\left(\gamma d^{A}+\frac{a^{* A}}{p^{A}+1+p^{A} d^{A}}\right) \pi-\left(\gamma d^{A}+\frac{a^{* A}}{p^{A}+2+p^{A} d^{A}}\right)(1-\pi) & \\
-\left(\gamma d^{* A}+\frac{a^{* A}}{p^{A}+1+p^{A} d^{* A}}\right) \pi-\left(\gamma d^{* A}+\frac{a^{* A}}{p^{A}+2+p^{A} d^{* A}}\right)(1-\pi) \geq & \text { if } p^{A}=2 \\
\geq-\left(\gamma d^{A}+\frac{a^{* A}}{p^{A}+1+p^{A} d^{A}}\right) \pi-\left(\gamma d^{A}+\frac{a^{* A}}{p^{A}+2+p^{A} d^{A}}\right)(1-\pi) & \end{cases} \\
& \left\{\begin{array}{l}
-\left(\gamma d^{* B}+\frac{1-a^{* A}}{p^{B}+1+p^{B} d^{* B}}\right) \pi-\left(\gamma d^{* B}+\frac{1-a^{* A}}{p^{B}+2+p^{B} d^{* B}}\right)(1-\pi) \geq \\
\geq-\left(\gamma d^{B}+\frac{1-a^{* A}}{p^{B}+1+p^{B} d^{B}}\right) \pi-\left(\gamma d^{B}+\frac{1-a^{* A}}{p^{B}+2+p^{B} d^{B}}\right)(1-\pi) \\
-\left(\gamma d^{* B}+\frac{1-a^{* A}}{p^{B}+1+p^{B} d^{* B}}\right) \pi-\left(\gamma d^{* B}+\frac{1-a^{* A}}{p^{B}+2+p^{B} d^{* B}}\right)(1-\pi) \geq \\
\geq-\left(\gamma d^{B}+\frac{1-a^{* A}}{p^{B}+1+p^{B} d^{B}}\right) \pi-\left(\gamma d^{B}+\frac{1-a^{* A}}{p^{B}+2+p^{B} d^{B}}\right)(1-\pi)
\end{array}\right.
\end{aligned}
$$

which is impossible. 


\section{Case 4.3}

$$
\delta^{A}\left(p^{A}\right)=\left\{\begin{array}{lll}
1 & \text { if } & p^{A}=1 \\
1 & \text { if } & p^{A}=2
\end{array} \quad \text { and } \delta^{B}\left(p^{B}\right)=\left\{\begin{array}{lll}
0 & \text { if } & p^{B}=1 \\
0 & \text { if } & p^{B}=2
\end{array}\right.\right.
$$

Being the game symmetric, we get exactly the same conclusion of the previous paragraph.

\section{Case 4.4}

$$
\begin{gathered}
\delta^{A}\left(p^{A}\right)=\left\{\begin{array}{ll}
1 & \text { if } p^{A}=1 \\
1 & \text { if } p^{A}=2
\end{array} \text { and } \delta^{B}\left(p^{B}\right)=\left\{\begin{array}{lll}
1 & \text { if } p^{B}=1 \\
1 & \text { if } p^{B}=2
\end{array}\right.\right. \\
\mu^{A}=\mu\left(p^{A}=1 \mid d^{A}\right)=\left\{\begin{array}{ll}
\pi & \text { if } d^{A}=0 \\
\pi & \text { if } d^{A}=1
\end{array} \text { and } \mu^{B}=\mu\left(p^{B}=1 \mid d^{B}\right)= \begin{cases}\pi & \text { if } d^{B}=0 \\
\pi & \text { if } d^{B}=1\end{cases} \right.
\end{gathered}
$$

- when $\left(d^{A}=0, d^{B}=0\right)$, then

$$
\begin{gathered}
E\left(\frac{p^{A} a}{p^{A}+p^{B}+p^{A} d^{A}}\right)+E\left(\frac{p^{B}(1-a)}{p^{A}+p^{B}+p^{B} d^{B}}\right)= \\
=\frac{a \pi^{2}}{2}+\frac{a \pi(1-\pi)}{3}+\frac{2 a(1-\pi) \pi}{3}+\frac{a(1-\pi)^{2}}{2}+ \\
+\frac{(1-a) \pi^{2}}{2}+\frac{2(1-a) \pi(1-\pi)}{3}+\frac{(1-a)(1-\pi) \pi}{3}+\frac{(1-a)(1-\pi)^{2}}{2} \Rightarrow \\
\Rightarrow a^{* A}\left(d^{A}=0, d^{B}=0\right) \in[0,1]
\end{gathered}
$$

- when $\left(d^{A}=0, d^{B}=1\right)$, then

$$
\begin{gathered}
E\left(\frac{p^{A} a}{p^{A}+p^{B}+p^{A} d^{A}}\right)+E\left(\frac{p^{B}(1-a)}{p^{A}+p^{B}+p^{B} d^{B}}\right)= \\
=\frac{a \pi^{2}}{2}+\frac{a \pi(1-\pi)}{3}+\frac{2 a(1-\pi) \pi}{3}+\frac{a(1-\pi)^{2}}{2}+ \\
+\frac{(1-a) \pi^{2}}{3}+\frac{2(1-a) \pi(1-\pi)}{5}+\frac{(1-a)(1-\pi) \pi}{4}+\frac{(1-a)(1-\pi)^{2}}{3} \Rightarrow \\
\Rightarrow a^{* A}\left(d^{A}=0, d^{B}=1\right)=1
\end{gathered}
$$

- when $\left(d^{A}=1, d^{B}=0\right)$, then

$$
\begin{gathered}
E\left(\frac{p^{A} a}{p^{A}+p^{B}+p^{A} d^{A}}\right)+E\left(\frac{p^{B}(1-a)}{p^{A}+p^{B}+p^{B} d^{B}}\right)= \\
=\frac{a \pi^{2}}{3}+\frac{a \pi(1-\pi)}{4}+\frac{2 a(1-\pi) \pi}{5}+\frac{a(1-\pi)^{2}}{3}+ \\
+\frac{(1-a) \pi^{2}}{2}+\frac{2(1-a) \pi(1-\pi)}{3}+\frac{(1-a)(1-\pi) \pi}{3}+\frac{(1-a)(1-\pi)^{2}}{2} \Rightarrow \\
\Rightarrow a^{* A}\left(d^{A}=1, d^{B}=0\right)=0
\end{gathered}
$$


- when $\left(d^{A}=1, d^{B}=1\right)$, then

$$
\begin{gathered}
E\left(\frac{p^{A} a}{p^{A}+p^{B}+p^{A} d^{A}}\right)+E\left(\frac{p^{B}(1-a)}{p^{A}+p^{B}+p^{B} d^{B}}\right)= \\
=\frac{a \pi^{2}}{3}+\frac{a \pi(1-\pi)}{4}+\frac{2 a(1-\pi) \pi}{5}+\frac{a(1-\pi)^{2}}{3}+ \\
+\frac{(1-a) \pi^{2}}{3}+\frac{2(1-a) \pi(1-\pi)}{5}+\frac{(1-a)(1-\pi) \pi}{4}+\frac{(1-a)(1-\pi)^{2}}{3} \Rightarrow \\
\Rightarrow a^{* A}\left(d^{A}=1, d^{B}=1\right) \in[0,1]
\end{gathered}
$$

To conclude

$$
a^{* A}\left(d^{A}, d^{B}\right) \in\left\{\begin{array}{ccc}
{[0,1]} & \text { if } \quad\left(d^{A}=0, d^{B}=0\right) \\
\{1\} & \text { if } \quad\left(d^{A}=0, d^{B}=1\right) \\
\{0\} & \text { if } \quad\left(d^{A}=1, d^{B}=0\right) \\
{[0,1]} & \text { if } \quad\left(d^{A}=1, d^{B}=1\right)
\end{array}\right.
$$

First stage: the governments' rational choices

$$
\begin{gathered}
\delta^{A}\left(p^{A}\right)=\left\{\begin{array}{ll}
1 & \text { if } p^{A}=1 \\
1 & \text { if } p^{A}=2
\end{array} \text { and } \delta^{B}\left(p^{B}\right)=\left\{\begin{array}{lll}
1 & \text { if } p^{B}=1 \\
1 & \text { if } p^{B}=2
\end{array}\right.\right. \\
a^{* A}\left(d^{A}, d^{B}\right) \in\left\{\begin{array}{cc}
{[0,1]} & \text { if }\left(d^{A}=0, d^{B}=0\right) \\
\{1\} & \text { if }\left(d^{A}=0, d^{B}=1\right) \\
\{0\} & \text { if }\left(d^{A}=1, d^{B}=0\right) \\
{[0,1]} & \text { if }\left(d^{A}=1, d^{B}=1\right)
\end{array}\right.
\end{gathered}
$$

are part of a PBE if and only if

$$
\begin{aligned}
& \left\{\begin{array}{l}
-\left(\gamma d^{* A}+\frac{a^{* A}}{p^{A}+1+p^{A} d^{* A}}\right) \pi-\left(\gamma d^{* A}+\frac{a^{* A}}{p^{A}+2+p^{A} d^{* A}}\right)(1-\pi) \geq \\
\geq-\left(\gamma d^{A}+\frac{a^{* A}}{p^{A}+1+p^{A} d^{A}}\right) \pi-\left(\gamma d^{A}+\frac{a^{* A}}{p^{A}+2+p^{A} d^{A}}\right)(1-\pi) \\
-\left(\gamma d^{* A}+\frac{a^{* A}}{p^{A}+1+p^{A} d^{* A}}\right) \pi-\left(\gamma d^{* A}+\frac{a^{* A}}{p^{A}+2+p^{A} d^{* A}}\right)(1-\pi) \geq \\
\geq-\left(\gamma d^{A}+\frac{a^{* A}}{p^{A}+1+p^{A} d^{A}}\right) \pi-\left(\gamma d^{A}+\frac{a^{* A}}{p^{A}+2+p^{A} d^{A}}\right)(1-\pi)
\end{array}\right. \\
& \left\{\begin{array}{l}
-\left(\gamma d^{* B}+\frac{1-a^{* A}}{p^{B}+1+p^{B} d^{* B}}\right) \pi-\left(\gamma d^{* B}+\frac{1-a^{* A}}{p^{B}+2+p^{B} d^{* B}}\right)(1-\pi) \geq \\
\geq-\left(\gamma d^{B}+\frac{1-a^{* A}}{p^{B}+1+p^{B} d^{B}}\right) \pi-\left(\gamma d^{B}+\frac{1-a^{* A}}{p^{B}+2+p^{B} d^{B}}\right)(1-\pi) \\
-\left(\gamma d^{* B}+\frac{1-a^{* A}}{p^{B}+1+p^{B} d^{* B}}\right) \pi-\left(\gamma d^{* B}+\frac{1-a^{* A}}{p^{B}+2+p^{B} d^{* B}}\right)(1-\pi) \geq \\
\geq-\left(\gamma d^{B}+\frac{1-a^{* A}}{p^{B}+1+p^{B} d^{B}}\right) \pi-\left(\gamma d^{B}+\frac{1-a^{* A}}{p^{B}+2+p^{B} d^{B}}\right)(1-\pi)
\end{array}\right.
\end{aligned}
$$

which are possible when

$\gamma \in\left[0, \frac{1}{12} \pi+\frac{1}{4}-\frac{1}{30} a^{* A} \pi-\frac{1}{6} a^{* A}\right] \cap\left[0, \frac{1}{12} \pi+\frac{1}{4}-\frac{1}{30}\left(1-a^{* A}\right) \pi-\frac{1}{6}\left(1-a^{* A}\right)\right]$.

Now, we are able to characterize the entire set of equilibria. 
Proposition 6 The counter-terrorism game with full decentralization has a set of pure strategy Perfect Bayesian Equilibria consistent with Passive Updating that depend on $\gamma$ and $\pi$ :

1. When

$$
\gamma \in\left[0, \frac{1}{12} \pi+\frac{1}{4}-\frac{1}{30} a^{*} \pi-\frac{1}{6} a^{*}\right] \cap\left[0, \frac{1}{12} \pi+\frac{1}{4}-\frac{1}{30}\left(1-a^{*}\right) \pi-\frac{1}{6}\left(1-a^{*}\right)\right]
$$

with $a^{*} \in[0,1]$, there is a PBE such that it is sequentially rational to play

$$
\begin{gathered}
\delta^{A *}\left(p^{A}\right)=\left\{\begin{array}{ll}
1 & \text { if } p^{A}=1 \\
1 & \text { if } p^{A}=2
\end{array} \text { and } \delta^{B *}\left(p^{B}\right)= \begin{cases}1 & \text { if } p^{B}=1 \\
1 & \text { if } p^{B}=2\end{cases} \right. \\
a^{A *}\left(d^{A}, d^{B}\right) \in\left\{\begin{array}{ccc}
{[0,1]} & \text { if }\left(d^{A}=0, d^{B}=0\right) \\
\{1\} & \text { if }\left(d^{A}=0, d^{B}=1\right) \\
\{0\} & \text { if }\left(d^{A}=1, d^{B}=0\right) \\
{[0,1]} & \text { if }\left(d^{A}=1, d^{B}=1\right) .
\end{array}\right.
\end{gathered}
$$

2. When

$$
\begin{aligned}
\gamma \in[ & {\left[\frac{1}{3} \pi+\frac{a^{*}}{4}-\frac{9 a^{*}}{20} \pi, \frac{1}{2} \pi+\frac{a^{*}}{3}-\frac{2 a^{*}}{3} \pi\right] \cap } \\
& \cap\left[-\frac{7}{60} \pi+\frac{1}{4}-\frac{a^{*}}{4}+\frac{9 a^{*}}{20} \pi,-\frac{1}{6} \pi+\frac{a^{*}}{3} \pi+\frac{1}{3}\right]
\end{aligned}
$$

and $a^{*} \in[0,1]$, there is a PBE such that it is sequentially rational to play

$$
\begin{gathered}
\delta^{A *}\left(p^{A}\right)=\left\{\begin{array}{ll}
1 & \text { if } p^{A}=1 \\
0 & \text { if } p^{A}=2
\end{array} \text { and } \delta^{B *}\left(p^{B}\right)=\left\{\begin{array}{lll}
1 & \text { if } p^{B}=1 \\
0 & \text { if } p^{B}=2
\end{array}\right.\right. \\
a^{A *}\left(d^{A}, d^{B}\right) \in\left\{\begin{array}{ccc}
{[0,1]} & \text { if }\left(d^{A}=0, d^{B}=0\right) \\
\{1\} & \text { if }\left(d^{A}=0, d^{B}=1\right) \\
\{0\} & \text { if }\left(d^{A}=1, d^{B}=0\right) \\
{[0,1]} & \text { if }\left(d^{A}=1, d^{B}=1\right) .
\end{array}\right.
\end{gathered}
$$

3. When

$$
\gamma \in\left[\left(\frac{7}{12}\right) \pi ;\left(\frac{5}{6}\right) \pi\right]
$$

there are two PBE such that it is sequentially rational to play

(a)

$$
\begin{gathered}
\delta^{A *}\left(p^{A}\right)=\left\{\begin{array}{ll}
0 & \text { if } p^{A}=1 \\
0 & \text { if } p^{A}=2
\end{array} \text { and } \delta^{B *}\left(p^{B}\right)= \begin{cases}1 & \text { if } p^{B}=1 \\
0 & \text { if } p^{B}=2\end{cases} \right. \\
a^{A *}\left(d^{A}, d^{B}\right) \in \begin{cases}\{0\} & \text { if }\left(d^{A}=0, d^{B}=0\right) \\
\{1\} & \text { if }\left(d^{A}=0, d^{B}=1\right) \\
\{0\} & \text { if }\left(d^{A}=1, d^{B}=0\right) \\
\{1\} & \text { if }\left(d^{A}=1, d^{B}=1\right)\end{cases}
\end{gathered}
$$


(b)

$$
\begin{gathered}
\delta^{A *}\left(p^{A}\right)=\left\{\begin{array}{ll}
1 & \text { if } p^{A}=1 \\
0 & \text { if } p^{A}=2
\end{array} \text { and } \delta^{B *}\left(p^{B}\right)= \begin{cases}0 & \text { if } p^{B}=1 \\
0 & \text { if } p^{B}=2\end{cases} \right. \\
a^{A *}\left(d^{A}, d^{B}\right) \in \begin{cases}\{1\} & \text { if }\left(d^{A}=0, d^{B}=0\right) \\
\{1\} & \text { if }\left(d^{A}=0, d^{B}=1\right) \\
\{0\} & \text { if }\left(d^{A}=1, d^{B}=0\right) \\
\{0\} & \text { if }\left(d^{A}=1, d^{B}=1\right)\end{cases}
\end{gathered}
$$

4. When

$$
\gamma \in\left[\left(\frac{a^{* A}}{2}\right) \pi+\left(\frac{a^{* A}}{3}\right)(1-\pi), 1\right] \cap\left[\left(\frac{1-a^{* A}}{2}\right) \pi+\left(\frac{1-a^{* A}}{3}\right)(1-\pi), 1\right]
$$

there is a PBE such that it is sequentially rational to play

$$
\begin{gathered}
\delta^{A *}\left(p^{A}\right)=\left\{\begin{array}{ll}
0 & \text { if } p^{A}=1 \\
0 & \text { if } p^{A}=2
\end{array} \text { and } \delta^{B *}\left(p^{B}\right)=\left\{\begin{array}{lll}
0 & \text { if } p^{B}=1 \\
0 & \text { if } p^{B}=2
\end{array}\right.\right. \\
a^{* A}\left(d^{A}, d^{B}\right) \in\left\{\begin{array}{ccc}
{[0,1]} & \text { if }\left(d^{A}=0, d^{B}=0\right) \\
\{1\} & \text { if }\left(d^{A}=0, d^{B}=1\right) \\
\{0\} & \text { if }\left(d^{A}=1, d^{B}=0\right) \\
{[0,1]} & \text { if }\left(d^{A}=1, d^{B}=1\right) .
\end{array}\right.
\end{gathered}
$$

\section{Appendix D: Perfect Bayesian Equilibria with Intelligence Cooperation}

Consider the 16 possible combination of beliefs and the consequent sequential rational choice of the countries (lemma 1). We consider only pure strategy PBE

1. Suppose

$$
\left\{\begin{array}{c}
\mu^{2,1 \mid 0,0} \geq \mu^{1,2 \mid 0,0} \\
\mu^{2,1 \mid 1,0} \geq \frac{15 \mu^{1,2 \mid 1,0}+10}{14} \\
\mu^{2,1 \mid 0,1} \geq \frac{14 \mu^{1,2 \mid 0,1}-10}{15} \\
\mu^{2,1 \mid 1,1} \geq \mu^{1,2 \mid 1,1}
\end{array}\right.
$$

Hence

(a) in $\left(p^{A}, p^{B}\right)=(1,1)$ then

$$
\begin{aligned}
& -\left(\gamma d^{* A}+\frac{a^{*}}{2+d^{* A}}\right) \geq-\left(\gamma d^{A}+\frac{a^{*}}{2+d^{A}}\right) \Rightarrow \delta^{A *}\left(d^{B} \mid\left(p^{A}, p^{B}\right)=(1,1)\right)=\left\{\begin{array}{lll}
1 & \text { if } \gamma \in\left[0, \frac{1}{6}\right. \\
0 & \text { if } \gamma \in\left[\frac{1}{6}, 1\right.
\end{array}\right] \\
& -\left(\gamma d^{* B}+\frac{1-a^{*}}{2+d^{* B}}\right) \geq-\left(\gamma d^{B}+\frac{1-a^{*}}{2+d^{B}}\right) \Rightarrow \delta^{B *}\left(d^{A} \mid\left(p^{A}, p^{B}\right)=(1,1)\right)=0
\end{aligned}
$$


(b) in $\left(p^{A}, p^{B}\right)=(2,1)$ then

$$
\begin{aligned}
& -\left(\gamma d^{* A}+\frac{a^{*}}{3+2 d^{* A}}\right) \geq-\left(\gamma d^{A}+\frac{a^{*}}{3+2 d^{A}}\right) \Rightarrow d^{A *}\left(d^{B} \mid\left(p^{A}, p^{B}\right)=(1,1)\right)=\left\{\begin{array}{lll}
1 & \text { if } \gamma \in\left[0, \frac{2}{15}\right. \\
0 & \text { if } \gamma \in\left[\frac{2}{15}, 1\right]
\end{array}\right] \\
& -\left(\gamma d^{* B}+\frac{1-a^{*}}{3+d^{* B}}\right) \geq-\left(\gamma d^{B}+\frac{1-a^{*}}{3+d^{B}}\right) \Rightarrow d^{B *}\left(d^{A} \mid\left(p^{A}, p^{B}\right)=(1,1)\right)=0
\end{aligned}
$$

(c) in $\left(p^{A}, p^{B}\right)=(1,2)$ then

$$
\begin{aligned}
& -\left(\gamma d^{* A}+\frac{a^{*}}{3+d^{* A}}\right) \geq-\left(\gamma d^{A}+\frac{a^{*}}{3+d^{A}}\right) \Rightarrow \delta^{A *}\left(d^{B} \mid\left(p^{A}, p^{B}\right)=(1,1)\right)=\left\{\begin{array}{ll}
1 & \text { if } \gamma \in\left[0, \frac{1}{12}\right] \\
0 & \text { if } \gamma \in\left[\frac{1}{12}, 1\right.
\end{array}\right] \\
& -\left(\gamma d^{* B}+\frac{1-a^{*}}{3+2 d^{* B}}\right) \geq-\left(\gamma d^{B}+\frac{1-a^{*}}{3+2 d^{B}}\right) \Rightarrow \delta^{B *}\left(d^{A} \mid\left(p^{A}, p^{B}\right)=(1,1)\right)=0
\end{aligned}
$$

(d) in $\left(p^{A}, p^{B}\right)=(2,2)$ then

$$
\begin{aligned}
& -\left(\gamma d^{* A}+\frac{a^{*}}{4+2 d^{* A}}\right) \geq-\left(\gamma d^{A}+\frac{a^{*}}{4+2 d^{A}}\right) \Rightarrow \delta^{A *}\left(d^{B} \mid\left(p^{A}, p^{B}\right)=(1,1)\right)=\left\{\begin{array}{lll}
1 & \text { if } \gamma \in\left[0, \frac{1}{12}\right] \\
0 & \text { if } \gamma \in\left[\frac{1}{12}, 1\right]
\end{array}\right] \\
& -\left(\gamma d^{* B}+\frac{1-a^{*}}{4+2 d^{* B}}\right) \geq-\left(\gamma d^{B}+\frac{1-a^{*}}{4+2 d^{B}}\right) \Rightarrow \delta^{B *}\left(d^{A} \mid\left(p^{A}, p^{B}\right)=(1,1)\right)=0
\end{aligned}
$$

So we conclude that in these beliefs regions, there are the following possible $\mathrm{PBE}$

i. if $\gamma \in\left[0, \frac{1}{12}\right]$

$$
\left\{d^{A *}\left(p^{A}, p^{B}\right), d^{B *}\left(p^{A}, p^{B}\right)\right\}= \begin{cases}\{1,0\} & \text { if }\left(p^{A}, p^{B}\right)=(1,1) \\ \{1,0\} & \text { if }\left(p^{A}, p^{B}\right)=(2,1) \\ \{1,0\} & \text { if }\left(p^{A}, p^{B}\right)=(1,2) \\ \{1,0\} & \text { if }\left(p^{A}, p^{B}\right)=(2,2)\end{cases}
$$

ii. if $\gamma \in\left[\frac{1}{12}, \frac{2}{15}\right]$

$$
\left\{d^{A *}\left(p^{A}, p^{B}\right), d^{B *}\left(p^{A}, p^{B}\right)\right\}= \begin{cases}\{1,0\} & \text { if }\left(p^{A}, p^{B}\right)=(1,1) \\ \{1,0\} & \text { if }\left(p^{A}, p^{B}\right)=(2,1) \\ \{0,0\} & \text { if }\left(p^{A}, p^{B}\right)=(1,2) \\ \{0,0\} & \text { if }\left(p^{A}, p^{B}\right)=(2,2)\end{cases}
$$

iii. if $\gamma \in\left[\frac{2}{15}, \frac{1}{6}\right]$

$$
\left\{d^{A *}\left(p^{A}, p^{B}\right), d^{B *}\left(p^{A}, p^{B}\right)\right\}= \begin{cases}\{1,0\} & \text { if }\left(p^{A}, p^{B}\right)=(1,1) \\ \{0,0\} & \text { if }\left(p^{A}, p^{B}\right)=(2,1) \\ \{0,0\} & \text { if }\left(p^{A}, p^{B}\right)=(1,2) \\ \{0,0\} & \text { if }\left(p^{A}, p^{B}\right)=(2,2)\end{cases}
$$


iv. if $\gamma \in\left[\frac{1}{6}, 1\right]$

$$
\left\{d^{A *}\left(p^{A}, p^{B}\right), d^{B *}\left(p^{A}, p^{B}\right)\right\}= \begin{cases}\{0,0\} & \text { if }\left(p^{A}, p^{B}\right)=(1,1) \\ \{0,0\} & \text { if }\left(p^{A}, p^{B}\right)=(2,1) \\ \{0,0\} & \text { if }\left(p^{A}, p^{B}\right)=(1,2) \\ \{0,0\} & \text { if }\left(p^{A}, p^{B}\right)=(2,2)\end{cases}
$$

Now, we have to go back to terrorists' beliefs

- when $\gamma \in\left[0, \frac{1}{12}\right]$ then $\mu^{2,1 \mid 1,0}=(1-\pi) \pi$ which means that $\mu^{2,1 \mid 1,0} \geq \frac{15 \mu^{1,2 \mid 1,0}+10}{14}$ is not satisfied, hence we get no PBE.

- when $\gamma \in\left[\frac{1}{12}, \frac{2}{15}\right]$ then $\mu^{2,1 \mid 1,0}=\frac{(1-\pi) \pi}{\pi^{2}+(1-\pi) \pi}, \mu^{1,2 \mid 1,0}=0 \quad$ which means that $\mu^{2,1 \mid 1,0} \geq \frac{15 \mu^{1,2 \mid 1,0}+10}{14} \Leftrightarrow \pi \leq \frac{2}{7}$. Moreover $\mu^{2,1 \mid 0,0}=0$, $\mu^{1,2 \mid 0,0}=\frac{(1-\pi) \pi}{(1-\pi) \pi+(1-\pi)^{2}} \quad$ which means that $\mu^{2,1 \mid 0,0} \geq \mu^{1,2 \mid 0,0} \quad$ is impossible.

- when $\gamma \in\left[\frac{2}{15}, \frac{1}{6}\right]$ then $\mu^{2,1 \mid 1,0}=0, \mu^{1,2 \mid 1,0}=0$ which means that $\mu^{2,1 \mid 1,0} \geq \frac{15 \mu^{1,2 \mid 1,0}+10}{14}$ is impossible.

- when $\gamma \in\left[\frac{1}{6}, 1\right]$ then $\mu^{2,1 \mid 0,0}=(1-\pi) \pi, \mu^{1,2 \mid 0,0}=(1-\pi) \pi$ which means that $\mu^{2,1 \mid 0,0} \geq \mu^{1,2 \mid 0,0}$ is satisfied.

2. Suppose

$$
\left\{\begin{array}{c}
\mu^{2,1 \mid 0,0} \geq \mu^{1,2 \mid 0,0} \\
\mu^{2,1 \mid 1,0} \geq \frac{15 \mu^{1,2 \mid 1,0}+10}{14} \\
\mu^{2,1 \mid 0,1} \geq \frac{14 \mu^{1,2 \mid 0,1}-10}{15} \\
\mu^{2,1 \mid 1,1} \leq \mu^{1,2 \mid 1,1}
\end{array}\right.
$$

Following the scheme of point 1 , we conclude that in these beliefs regions there are the following possible PBE

(a) i. if $\gamma \in\left[0, \frac{1}{12}\right]$

$$
\left\{d^{A *}\left(p^{A}, p^{B}\right), d^{B *}\left(p^{A}, p^{B}\right)\right\}= \begin{cases}\{1,0\} & \text { if }\left(p^{A}, p^{B}\right)=(1,1) \\ \{1,0\} & \text { if }\left(p^{A}, p^{B}\right)=(2,1) \\ \{1,0\} & \text { if }\left(p^{A}, p^{B}\right)=(1,2) \\ \{1,0\} & \text { if }\left(p^{A}, p^{B}\right)=(2,2)\end{cases}
$$

ii. if $\gamma \in\left[\frac{1}{12}, \frac{2}{15}\right]$

$$
\left\{d^{A *}\left(p^{A}, p^{B}\right), d^{B *}\left(p^{A}, p^{B}\right)\right\}= \begin{cases}\{1,0\} & \text { if }\left(p^{A}, p^{B}\right)=(1,1) \\ \{1,0\} & \text { if }\left(p^{A}, p^{B}\right)=(2,1) \\ \{0,0\} & \text { if }\left(p^{A}, p^{B}\right)=(1,2) \\ \{0,0\} & \text { if }\left(p^{A}, p^{B}\right)=(2,2)\end{cases}
$$


iii. if $\gamma \in\left[\frac{2}{15}, \frac{1}{6}\right]$

$$
\left\{d^{A *}\left(p^{A}, p^{B}\right), d^{B *}\left(p^{A}, p^{B}\right)\right\}= \begin{cases}\{1,0\} & \text { if }\left(p^{A}, p^{B}\right)=(1,1) \\ \{0,0\} & \text { if }\left(p^{A}, p^{B}\right)=(2,1) \\ \{0,0\} & \text { if }\left(p^{A}, p^{B}\right)=(1,2) \\ \{0,0\} & \text { if }\left(p^{A}, p^{B}\right)=(2,2)\end{cases}
$$

iv. if $\gamma \in\left[\frac{1}{6}, 1\right]$

$$
\left\{d^{A *}\left(p^{A}, p^{B}\right), d^{B *}\left(p^{A}, p^{B}\right)\right\}= \begin{cases}\{0,0\} & \text { if }\left(p^{A}, p^{B}\right)=(1,1) \\ \{0,0\} & \text { if }\left(p^{A}, p^{B}\right)=(2,1) \\ \{0,0\} & \text { if }\left(p^{A}, p^{B}\right)=(1,2) \\ \{0,0\} & \text { if }\left(p^{A}, p^{B}\right)=(2,2)\end{cases}
$$

Now, we have to go back to terrorists' beliefs

- when $\gamma \in\left[0, \frac{1}{12}\right]$ then $\mu^{2,1 \mid 1,0}=(1-\pi) \pi, \mu^{1,2 \mid 1,0}=(1-\pi) \pi$ which means that $\mu^{2,1 \mid 1,0} \geq \frac{15 \mu^{1,2 \mid 1,0}+10}{14}$ is not satisfied, hence we get no PBE.

- when $\gamma \in\left[\frac{1}{12}, \frac{2}{15}\right]$ then $\mu^{2,1 \mid 0,0}=0, \mu^{1,2 \mid 0,0}=\frac{(1-\pi) \pi}{(1-\pi) \pi+(1-\pi)^{2}} \quad$ which means that $\mu^{2,1 \mid 0,0} \geq \mu^{1,2 \mid 0,0}$ is impossible.

- when $\gamma \in\left[\frac{2}{15}, \frac{1}{6}\right]$ then $\mu^{2,1 \mid 1,0}=0, \mu^{1,2 \mid 1,0}=0$ which means that $\mu^{2,1 \mid 1,0} \geq \frac{15 \mu^{1,2 \mid 1,0}+10}{14}$ is impossible.

- when $\gamma \in\left[\frac{1}{6}, 1\right]$ then $\mu^{2,1 \mid 0,0}=(1-\pi) \pi, \mu^{1,2 \mid 0,0}=(1-\pi) \pi$ which means that $\mu^{2,1 \mid 0,0} \geq \mu^{1,2 \mid 0,0}$ is satisfied.

3. Suppose

$$
\left\{\begin{array}{c}
\mu^{2,1 \mid 0,0} \geq \mu^{1,2 \mid 0,0} \\
\mu^{2,1 \mid 1,0} \geq \frac{15 \mu^{1,2 \mid 1,0}+10}{14} \\
\mu^{2,1 \mid 0,1} \leq \frac{14 \mu^{1,2 \mid 0,1}-10}{15} \\
\mu^{2,1 \mid 1,1} \geq \mu^{1,2 \mid 1,1}
\end{array}\right.
$$

Following the scheme of point 1 , we conclude that in these beliefs regions there are the following possible PBE

(a) i. if $\gamma \in\left[0, \frac{1}{12}\right]$

$$
\left\{d^{A *}\left(p^{A}, p^{B}\right), d^{B *}\left(p^{A}, p^{B}\right)\right\}= \begin{cases}\{1,0\} & \text { if }\left(p^{A}, p^{B}\right)=(1,1) \\ \{1,0\} & \text { if }\left(p^{A}, p^{B}\right)=(2,1) \\ \{1,0\} & \text { if }\left(p^{A}, p^{B}\right)=(1,2) \\ \{1,0\} & \text { if }\left(p^{A}, p^{B}\right)=(2,2)\end{cases}
$$

ii. if $\gamma \in\left[\frac{1}{12}, \frac{2}{15}\right]$

$$
\left\{d^{A *}\left(p^{A}, p^{B}\right), d^{B *}\left(p^{A}, p^{B}\right)\right\}= \begin{cases}\{1,0\} & \text { if }\left(p^{A}, p^{B}\right)=(1,1) \\ \{1,0\} & \text { if }\left(p^{A}, p^{B}\right)=(2,1) \\ \{1,0\} & \text { if }\left(p^{A}, p^{B}\right)=(1,2) \\ \{0,0\} & \text { if }\left(p^{A}, p^{B}\right)=(2,2)\end{cases}
$$


iii. if $\gamma \in\left[\frac{2}{15}, \frac{1}{6}\right]$

$$
\left\{d^{A *}\left(p^{A}, p^{B}\right), d^{B *}\left(p^{A}, p^{B}\right)\right\}= \begin{cases}\{1,0\} & \text { if }\left(p^{A}, p^{B}\right)=(1,1) \\ \{0,0\} & \text { if }\left(p^{A}, p^{B}\right)=(2,1) \\ \{0,0\} & \text { if }\left(p^{A}, p^{B}\right)=(1,2) \\ \{0,0\} & \text { if }\left(p^{A}, p^{B}\right)=(2,2)\end{cases}
$$

iv. if $\gamma \in\left[\frac{1}{6}, 1\right]$

$$
\left\{d^{A *}\left(p^{A}, p^{B}\right), d^{B *}\left(p^{A}, p^{B}\right)\right\}= \begin{cases}\{0,0\} & \text { if }\left(p^{A}, p^{B}\right)=(1,1) \\ \{0,0\} & \text { if }\left(p^{A}, p^{B}\right)=(2,1) \\ \{0,0\} & \text { if }\left(p^{A}, p^{B}\right)=(1,2) \\ \{0,0\} & \text { if }\left(p^{A}, p^{B}\right)=(2,2)\end{cases}
$$

Now, we have to go back to terrorists' beliefs. Hence

- when $\gamma \in\left[0, \frac{1}{12}\right]$ then $\mu^{2,1 \mid 1,0}=(1-\pi) \pi, \mu^{1,2 \mid 1,0}=(1-\pi) \pi$ which means that $\mu^{2,1 \mid 1,0} \geq \frac{15 \mu^{1,2 \mid 1,0}+10}{14}$ is not satisfied, hence we get no PBE.

- when $\gamma \in\left[\frac{1}{12}, \frac{2}{15}\right]$ then $\mu^{2,1 \mid 1,0}=\frac{(1-\pi) \pi}{\pi^{2}+2(1-\pi) \pi}, \quad \mu^{1,2 \mid 1,0}=\frac{(1-\pi) \pi}{\pi^{2}+2(1-\pi) \pi}$ which means that $\mu^{2,1 \mid 1,0} \geq \frac{15 \mu^{1,2 \mid 1,0}+10}{14}$ is impossible.

- when $\gamma \in\left[\frac{2}{15}, \frac{1}{6}\right]$ then $\mu^{2,1 \mid 1,0}=0, \mu^{1,2 \mid 1,0}=0$ which means that $\mu^{2,1 \mid 1,0} \geq \frac{15 \mu^{1,2 \mid 1,0}+10}{14}$ is impossible.

- when $\gamma \in\left[\frac{1}{6}, 1\right]$ then $\mu^{2,1 \mid 0,0}=(1-\pi) \pi, \mu^{1,2 \mid 0,0}=(1-\pi) \pi$ which means that $\mu^{2,1 \mid 0,0} \geq \mu^{1,2 \mid 0,0}$ is satisfied.

4. Suppose

$$
\left\{\begin{array}{c}
\mu^{2,1 \mid 0,0} \geq \mu^{1,2 \mid 0,0} \\
\mu^{2,1 \mid 1,0} \leq \frac{15 \mu^{1,2 \mid 1,0}+10}{14} \\
\mu^{2,1 \mid 0,1} \geq \frac{14 \mu^{1,2 \mid 0,1}-10}{15} \\
\mu^{2,1 \mid 1,1} \geq \mu^{1,2 \mid 1,1}
\end{array}\right.
$$

Following the scheme of point 1 , we conclude that in these beliefs regions there are the following possible $\mathrm{PBE}$

(a) i. if $\gamma \in\left[0, \frac{1}{12}\right]$

$$
\left\{d^{A *}\left(p^{A}, p^{B}\right), d^{B *}\left(p^{A}, p^{B}\right)\right\}= \begin{cases}\{1,1\} & \text { if }\left(p^{A}, p^{B}\right)=(1,1) \\ \{1,1\} & \text { if }\left(p^{A}, p^{B}\right)=(2,1) \\ \{1,1\} & \text { if }\left(p^{A}, p^{B}\right)=(1,2) \\ \{1,1\} & \text { if } \quad\left(p^{A}, p^{B}\right)=(2,2)\end{cases}
$$

ii. if $\gamma \in\left[\frac{1}{2}, 1\right]$

$$
\left\{d^{A *}\left(p^{A}, p^{B}\right), d^{B *}\left(p^{A}, p^{B}\right)\right\}= \begin{cases}\{0,0\} & \text { if }\left(p^{A}, p^{B}\right)=(1,1) \\ \{0,0\} & \text { if }\left(p^{A}, p^{B}\right)=(2,1) \\ \{0,0\} & \text { if }\left(p^{A}, p^{B}\right)=(1,2) \\ \{0,0\} & \text { if }\left(p^{A}, p^{B}\right)=(2,2)\end{cases}
$$


Now, we have to go back to terrorists' beliefs, considering pure strategy PBE:

- when $\gamma \in\left[0, \frac{1}{12}\right]$ then $\mu^{2,1 \mid 1,1}=(1-\pi) \pi, \mu^{1,2 \mid 1,1}=(1-\pi) \pi$ which means that $\mu^{2,1 \mid 1,1} \geq \mu^{1,2 \mid 1,1}$ is satisfied.

- when $\gamma \in\left[\frac{1}{2}, 1\right]$ then $\mu^{2,1 \mid 0,0}=(1-\pi) \pi, \mu^{1,2 \mid 0,0}=(1-\pi) \pi$ which means that $\mu^{2,1 \mid 0,0} \geq \mu^{1,2 \mid 0,0}$ is satisfied.

5. Suppose

$$
\left\{\begin{array}{c}
\mu^{2,1 \mid 0,0} \leq \mu^{1,2 \mid 0,0} \\
\mu^{2,1 \mid 1,0} \geq \frac{15 \mu^{1,2 \mid 1,0}+10}{14} \\
\mu^{2,1 \mid 0,1} \geq \frac{14 \mu^{1,2 \mid 0,1}-10}{15} \\
\mu^{2,1 \mid 1,1} \geq \mu^{1,2 \mid 1,1}
\end{array}\right.
$$

Following the scheme of point 1 , we conclude that in these beliefs regions there are the following possible PBE

(a) i. if $\gamma \in\left[\frac{1}{6}, \frac{1}{4}\right]$

$$
\left\{d^{A *}\left(p^{A}, p^{B}\right), d^{B *}\left(p^{A}, p^{B}\right)\right\}= \begin{cases}\{0,1\} & \text { if }\left(p^{A}, p^{B}\right)=(1,1) \\ \{0,1\} & \text { if }\left(p^{A}, p^{B}\right)=(2,1) \\ \{0,1\} & \text { if }\left(p^{A}, p^{B}\right)=(1,2) \\ \{0,1\} & \text { if }\left(p^{A}, p^{B}\right)=(2,2)\end{cases}
$$

ii. if $\gamma \in\left[\frac{1}{4}, \frac{1}{3}\right]$

$$
\left\{d^{A *}\left(p^{A}, p^{B}\right), d^{B *}\left(p^{A}, p^{B}\right)\right\}= \begin{cases}\{0,1\} & \text { if }\left(p^{A}, p^{B}\right)=(1,1) \\ \{0,1\} & \text { if }\left(p^{A}, p^{B}\right)=(2,1) \\ \{0,1\} & \text { if }\left(p^{A}, p^{B}\right)=(1,2) \\ \{0,0\} & \text { if }\left(p^{A}, p^{B}\right)=(2,2)\end{cases}
$$

iii. if $\gamma \in\left[\frac{1}{3}, \frac{1}{2}\right]$

$$
\left\{d^{A *}\left(p^{A}, p^{B}\right), d^{B *}\left(p^{A}, p^{B}\right)\right\}= \begin{cases}\{0,1\} & \text { if }\left(p^{A}, p^{B}\right)=(1,1) \\ \{0,0\} & \text { if }\left(p^{A}, p^{B}\right)=(2,1) \\ \{0,0\} & \text { if }\left(p^{A}, p^{B}\right)=(1,2) \\ \{0,0\} & \text { if }\left(p^{A}, p^{B}\right)=(2,2)\end{cases}
$$

iv. if $\gamma \in\left[\frac{1}{2}, 1\right]$

$$
\left\{d^{A *}\left(p^{A}, p^{B}\right), d^{B *}\left(p^{A}, p^{B}\right)\right\}= \begin{cases}\{0,0\} & \text { if }\left(p^{A}, p^{B}\right)=(1,1) \\ \{0,0\} & \text { if }\left(p^{A}, p^{B}\right)=(2,1) \\ \{0,0\} & \text { if }\left(p^{A}, p^{B}\right)=(1,2) \\ \{0,0\} & \text { if }\left(p^{A}, p^{B}\right)=(2,2)\end{cases}
$$

Now, we have to go back to terrorists' beliefs, considering pure strategy PBE only. 
- when $\gamma \in\left[\frac{1}{6}, \frac{1}{4}\right]$ then $\mu^{2,1 \mid 0,1}=(1-\pi) \pi, \mu^{1,2 \mid 0,1}=(1-\pi) \pi$ which means that $\mu^{2,1 \mid 0,1} \geq \frac{14 \mu^{1,2 \mid 0,1}-10}{15}$ is satisfied.

- when $\gamma \in\left[\frac{1}{4}, \frac{1}{3}\right]$ then $\mu^{2,1 \mid 0,1}=\frac{(1-\pi) \pi}{\pi^{2}+2(1-\pi) \pi}, \quad \mu^{1,2 \mid 0,1}=(1-\pi) \pi$ which means that $\mu^{2,1 \mid 0,1} \geq \frac{14 \mu^{1,2 \mid 0,1}-10}{15}$ is satisfied. Moreover $\mu^{2,1 \mid 0,0}=\mu^{1,2 \mid 0,0}=0$ which means that $\mu^{2,1 \mid 0,0} \leq \mu^{1,2 \mid 0,0}$ is satisfied.

- when $\gamma \in\left[\frac{1}{3}, \frac{1}{2}\right]$ then $\mu^{2,1 \mid 0,1}=\mu^{1,2 \mid 0,1}=0$ which means that $\mu^{2,1 \mid 0,1} \geq \frac{14 \mu^{1,2 \mid 0,1}-10}{15}$ is satisfied. Moreover $\mu^{2,1 \mid 0,0}=\mu^{1,2 \mid 0,0}=$ $\frac{(1-\pi) \pi}{2(1-\pi) \pi+(1-\pi)^{2}}$ which means that $\mu^{2,1 \mid 0,0} \leq \mu^{1,2 \mid 0,0}$ is satisfied.

- when $\gamma \in\left[\frac{1}{2}, 1\right]$ then $\mu^{2,1 \mid 0,0}=\mu^{1,2 \mid 0,0}=(1-\pi) \pi$ which means that $\mu^{2,1 \mid 0,0} \leq \mu^{1,2 \mid 0,0}$ is satisfied.

6. Suppose

$$
\left\{\begin{array}{c}
\mu^{2,1 \mid 0,0} \leq \mu^{1,2 \mid 0,0} \\
\mu^{2,1 \mid 1,0} \leq \frac{15 \mu^{1,2 \mid 1,0}+10}{14} \\
\mu^{2,1 \mid 0,1} \geq \frac{14 \mu^{1,2 \mid 0,1}-10}{15} \\
\mu^{2,1 \mid 1,1} \geq \mu^{1,2 \mid 1,1}
\end{array}\right.
$$

Following the scheme of point 1 , we conclude that in these beliefs regions there are the following possible $\mathrm{PBE}$

(a) i. if $\gamma \in\left[0, \frac{1}{12}\right]$

$$
\left\{d^{A *}\left(p^{A}, p^{B}\right), d^{B *}\left(p^{A}, p^{B}\right)\right\}= \begin{cases}\{1,1\} & \text { if }\left(p^{A}, p^{B}\right)=(1,1) \\ \{1,1\} & \text { if }\left(p^{A}, p^{B}\right)=(2,1) \\ \{1,1\} & \text { if }\left(p^{A}, p^{B}\right)=(1,2) \\ \{1,1\} & \text { if }\left(p^{A}, p^{B}\right)=(2,2)\end{cases}
$$

ii. if $\gamma \in\left[\frac{1}{12}, \frac{2}{15}\right]$

$$
\left\{d^{A *}\left(p^{A}, p^{B}\right), d^{B *}\left(p^{A}, p^{B}\right)\right\}= \begin{cases}\{1,1\} & \text { if }\left(p^{A}, p^{B}\right)=(1,1) \\ \{1,1\} & \text { if }\left(p^{A}, p^{B}\right)=(2,1) \\ \{0,1\} & \text { if }\left(p^{A}, p^{B}\right)=(1,2) \\ \{0,1\} & \text { if }\left(p^{A}, p^{B}\right)=(2,2)\end{cases}
$$

iii. if $\gamma \in\left[\frac{2}{15}, \frac{1}{6}\right]$

$$
\left\{d^{A *}\left(p^{A}, p^{B}\right), d^{B *}\left(p^{A}, p^{B}\right)\right\}= \begin{cases}\{1,1\} & \text { if }\left(p^{A}, p^{B}\right)=(1,1) \\ \{0,1\} & \text { if }\left(p^{A}, p^{B}\right)=(2,1) \\ \{0,1\} & \text { if }\left(p^{A}, p^{B}\right)=(1,2) \\ \{0,1\} & \text { if }\left(p^{A}, p^{B}\right)=(2,2)\end{cases}
$$

iv. if $\gamma \in\left[\frac{1}{6}, \frac{1}{4}\right]$

$$
\left\{d^{A *}\left(p^{A}, p^{B}\right), d^{B *}\left(p^{A}, p^{B}\right)\right\}= \begin{cases}\{0,1\} & \text { if }\left(p^{A}, p^{B}\right)=(1,1) \\ \{0,1\} & \text { if }\left(p^{A}, p^{B}\right)=(2,1) \\ \{0,1\} & \text { if }\left(p^{A}, p^{B}\right)=(1,2) \\ \{0,1\} & \text { if }\left(p^{A}, p^{B}\right)=(2,2)\end{cases}
$$


v. if $\gamma \in\left[\frac{1}{4}, \frac{1}{3}\right]$

$$
\left\{d^{A *}\left(p^{A}, p^{B}\right), d^{B *}\left(p^{A}, p^{B}\right)\right\}= \begin{cases}\{0,1\} & \text { if }\left(p^{A}, p^{B}\right)=(1,1) \\ \{0,1\} & \text { if }\left(p^{A}, p^{B}\right)=(2,1) \\ \{0,1\} & \text { if }\left(p^{A}, p^{B}\right)=(1,2) \\ \{0,0\} & \text { if }\left(p^{A}, p^{B}\right)=(2,2)\end{cases}
$$

vi. if $\gamma \in\left[\frac{1}{3}, \frac{1}{2}\right]$

$$
\left\{d^{A *}\left(p^{A}, p^{B}\right), d^{B *}\left(p^{A}, p^{B}\right)\right\}= \begin{cases}\{0,1\} & \text { if }\left(p^{A}, p^{B}\right)=(1,1) \\ \{0,0\} & \text { if }\left(p^{A}, p^{B}\right)=(2,1) \\ \{0,0\} & \text { if }\left(p^{A}, p^{B}\right)=(1,2) \\ \{0,0\} & \text { if }\left(p^{A}, p^{B}\right)=(2,2)\end{cases}
$$

vii. if $\gamma \in\left[\frac{1}{2}, 1\right]$

$$
\left\{d^{A *}\left(p^{A}, p^{B}\right), d^{B *}\left(p^{A}, p^{B}\right)\right\}= \begin{cases}\{0,0\} & \text { if } \quad\left(p^{A}, p^{B}\right)=(1,1) \\ \{0,0\} & \text { if } \quad\left(p^{A}, p^{B}\right)=(2,1) \\ \{0,0\} & \text { if }\left(p^{A}, p^{B}\right)=(1,2) \\ \{0,0\} & \text { if } \quad\left(p^{A}, p^{B}\right)=(2,2)\end{cases}
$$

Now, we have to go back to terrorists' beliefs, considering pure strategy PBE only:

- when $\gamma \in\left[0, \frac{1}{12}\right]$ then $\mu^{2,1 \mid 1,1}=\mu^{1,2 \mid 1,1}=(1-\pi) \pi$ which means that $\mu^{2,1 \mid 1,1} \geq \mu^{1,2 \mid 1,1}$ is satisfied.

- when $\gamma \in\left[\frac{1}{12}, \frac{2}{15}\right]$ then $\mu^{2,1 \mid 0,1}=0, \mu^{1,2 \mid 0,1}=\frac{(1-\pi) \pi}{(1-\pi) \pi+(1-\pi)^{2}}$ which means that

$\mu^{2,1 \mid 0,1} \geq \frac{14 \mu^{1,2 \mid 0,1}-10}{15} \Leftrightarrow \pi \leq \frac{12-\sqrt{144-140}}{14}=\frac{5}{7}$ or $\pi \geq \frac{12+\sqrt{144-140}}{14}=1$

so that it is satisfied when $\pi \in\left[0, \frac{5}{7}\right]$. Moreover $\mu^{2,1 \mid 1,1}=\frac{(1-\pi) \pi}{\pi^{2}+(1-\pi) \pi}$, $\mu^{1,2 \mid 1,1}=0$ which means that $\mu^{2,1 \mid 1,1} \geq \mu^{1,2 \mid 1,1}$ is satisfied.

- when $\gamma \in\left[\frac{2}{15}, \frac{1}{6}\right]$ then $\mu^{2,1 \mid 0,1}=\mu^{1,2 \mid 0,1}=\frac{(1-\pi) \pi}{2(1-\pi) \pi+(1-\pi)^{2}}$ which means that $\mu^{2,1 \mid 0,1} \geq \frac{14 \mu^{1,2 \mid 0,1}-10}{15}$ is satisfied. Moreover $\mu^{2,1 \mid 1,1}=$ $\mu^{1,2 \mid 1,1}=0$ which means that $\mu^{2,1 \mid 1,1} \geq \mu^{1,2 \mid 1,1}$ is satisfied.

- when $\gamma \in\left[\frac{1}{6}, \frac{1}{4}\right]$ then $\mu^{2,1 \mid 0,1}=\mu^{1,2 \mid 0,1}=(1-\pi) \pi$ which means that $\mu^{2,1 \mid 0,1} \geq \frac{14 \mu^{1,2 \mid 0,1}-10}{15}$ is satisfied.

- when $\gamma \in\left[\frac{1}{4}, \frac{1}{3}\right]$ then $\mu^{2,1 \mid 0,1}=\mu^{1,2 \mid 0,1}=\frac{(1-\pi) \pi}{\pi^{2}+2(1-\pi) \pi}$ which means that $\mu^{2,1 \mid 0,1} \geq \frac{14 \mu^{1,2 \mid 0,1}-10}{15}$ is satisfied. Moreover $\mu^{2,1 \mid 0,0}=$ $\mu^{1,2 \mid 0,0}=0$ which means that $\mu^{2,1 \mid 0,0} \leq \mu^{1,2 \mid 0,0}$ is satisfied. 
- when $\gamma \in\left[\frac{1}{3}, \frac{1}{2}\right]$ then $\mu^{2,1 \mid 0,1}=\mu^{1,2 \mid 0,1}=0$ which means that $\mu^{2,1 \mid 0,1} \geq \frac{14 \mu^{1,2 \mid 0,1}-10}{15}$ is satisfied. Moreover $\mu^{2,1 \mid 0,0}=\mu^{1,2 \mid 0,0}=$ $\frac{(1-\pi) \pi}{2(1-\pi) \pi+(1-\pi)^{2}}$ which means that $\mu^{2,1 \mid 0,0} \leq \mu^{1,2 \mid 0,0}$ is satisfied.

- when $\gamma \in\left[\frac{1}{2}, 1\right]$ then $\mu^{2,1 \mid 0,0}=\mu^{1,2 \mid 0,0}=(1-\pi) \pi$ which means that $\mu^{2,1 \mid 0,0} \leq \mu^{1,2 \mid 0,0}$ is satisfied.

7. Suppose

$$
\left\{\begin{array}{c}
\mu^{2,1 \mid 0,0} \leq \mu^{1,2 \mid 0,0} \\
\mu^{2,1 \mid 1,0} \geq \frac{15 \mu^{1,2 \mid 1,0}+10}{14} \\
\mu^{2,1 \mid 0,1} \leq \frac{14 \mu^{1,2 \mid 0,1}-10}{15} \\
\mu^{2,1 \mid 1,1} \geq \mu^{1,2 \mid 1,1}
\end{array}\right.
$$

Following the scheme of point 1 , we conclude that in these beliefs regions there are the following possible $\mathrm{PBE}$

(a) i. if $\gamma \in\left[0, \frac{1}{12}\right]$

$$
\left\{d^{A *}\left(p^{A}, p^{B}\right), d^{B *}\left(p^{A}, p^{B}\right)\right\}= \begin{cases}\{0,1\} & \text { if }\left(p^{A}, p^{B}\right)=(1,1) \\ \{0,1\} & \text { if }\left(p^{A}, p^{B}\right)=(2,1) \\ \{0,1\} & \text { if }\left(p^{A}, p^{B}\right)=(1,2) \\ \{0,1\} & \text { if }\left(p^{A}, p^{B}\right)=(2,2)\end{cases}
$$

ii. if $\gamma \in\left[\frac{1}{12}, \frac{2}{15}\right]$

$$
\left\{d^{A *}\left(p^{A}, p^{B}\right), d^{B *}\left(p^{A}, p^{B}\right)\right\}= \begin{cases}\{0,1\} & \text { if }\left(p^{A}, p^{B}\right)=(1,1) \\ \{0,0\} & \text { if }\left(p^{A}, p^{B}\right)=(2,1) \\ \{0,1\} & \text { if }\left(p^{A}, p^{B}\right)=(1,2) \\ \{0,0\} & \text { if }\left(p^{A}, p^{B}\right)=(2,2)\end{cases}
$$

iii. if $\gamma \in\left[\frac{2}{15}, \frac{1}{6}\right]$

$$
\left\{d^{A *}\left(p^{A}, p^{B}\right), d^{B *}\left(p^{A}, p^{B}\right)\right\}= \begin{cases}\{0,1\} & \text { if }\left(p^{A}, p^{B}\right)=(1,1) \\ \{0,0\} & \text { if }\left(p^{A}, p^{B}\right)=(2,1) \\ \{0,0\} & \text { if }\left(p^{A}, p^{B}\right)=(1,2) \\ \{0,0\} & \text { if }\left(p^{A}, p^{B}\right)=(2,2)\end{cases}
$$

iv. if $\gamma \in\left[\frac{1}{6}, 1\right]$

$$
\left\{d^{A *}\left(p^{A}, p^{B}\right), d^{B *}\left(p^{A}, p^{B}\right)\right\}= \begin{cases}\{0,0\} & \text { if }\left(p^{A}, p^{B}\right)=(1,1) \\ \{0,0\} & \text { if }\left(p^{A}, p^{B}\right)=(2,1) \\ \{0,0\} & \text { if }\left(p^{A}, p^{B}\right)=(1,2) \\ \{0,0\} & \text { if }\left(p^{A}, p^{B}\right)=(2,2)\end{cases}
$$

Now, back to terrorists' beliefs

- when $\gamma \in\left[0, \frac{1}{12}\right]$ then $\mu^{2,1 \mid 0,1}=\mu^{1,2 \mid 0,1}=(1-\pi) \pi$ which means that $\mu^{2,1 \mid 0,1} \leq \frac{14 \mu^{1,2 \mid 0,1}-10}{15}$ is impossible. 
- when $\gamma \in\left[\frac{1}{12}, \frac{2}{15}\right]$ then $\mu^{2,1 \mid 0,0}=1, \mu^{1,2 \mid 0,0}=0$ which means that $\mu^{2,1 \mid 0,0} \leq \mu^{1,2 \mid 0,0}$ is impossible.

- when $\gamma \in\left[\frac{2}{15}, \frac{1}{6}\right]$ then $\mu^{2,1 \mid 0,1}=\mu^{1,2 \mid 0,1}=0$ which means that $\mu^{2,1 \mid 0,1} \geq \frac{14 \mu^{1,2 \mid 0,1}-10}{15}$ never satisfied.

- when $\gamma \in\left[\frac{1}{6}, 1\right]$ then $\mu^{2,1 \mid 0,0}=\mu^{1,2 \mid 0,0}=(1-\pi) \pi$ which means that $\mu^{2,1 \mid 0,0} \leq \mu^{1,2 \mid 0,0}$ is satisfied.

8. Suppose

$$
\left\{\begin{array}{c}
\mu^{2,1 \mid 0,0} \leq \mu^{1,2 \mid 0,0} \\
\mu^{2,1 \mid 1,0} \geq \frac{15 \mu^{1,2 \mid 1,0}+10}{14} \\
\mu^{2,1 \mid 0,1} \geq \frac{14 \mu^{1,2 \mid 0,1}-10}{15} \\
\mu^{2,1 \mid 1,1} \leq \mu^{1,2 \mid 1,1}
\end{array}\right.
$$

Following the scheme of point 1 , we conclude that in these beliefs regions there are the following possible $\mathrm{PBE}$

(a) i. if $\gamma \in\left[\frac{1}{2}, 1\right]$

$$
\left\{d^{A *}\left(p^{A}, p^{B}\right), d^{B *}\left(p^{A}, p^{B}\right)\right\}= \begin{cases}\{0,0\} & \text { if }\left(p^{A}, p^{B}\right)=(1,1) \\ \{0,0\} & \text { if }\left(p^{A}, p^{B}\right)=(2,1) \\ \{0,0\} & \text { if }\left(p^{A}, p^{B}\right)=(1,2) \\ \{0,0\} & \text { if }\left(p^{A}, p^{B}\right)=(2,2)\end{cases}
$$

Now, we have to go back to terrorists' beliefs, considering pure strategy PBE only. Hence

- when $\gamma \in\left[\frac{1}{2}, 1\right]$ then $\mu^{2,1 \mid 0,0}=\mu^{1,2 \mid 0,0}=(1-\pi) \pi$ which means that $\mu^{2,1 \mid 0,0} \leq \mu^{1,2 \mid 0,0}$ is satisfied.

9. Suppose

$$
\left\{\begin{array}{c}
\mu^{2,1 \mid 0,0} \geq \mu^{1,2 \mid 0,0} \\
\mu^{2,1 \mid 1,0} \leq \frac{15 \mu^{1,2 \mid 1,0}+10}{14} \\
\mu^{2,1 \mid 0,1} \leq \frac{14 \mu^{1,2 \mid 0,1}-10}{15} \\
\mu^{2,1 \mid 1,1} \geq \mu^{1,2 \mid 1,1}
\end{array}\right.
$$

Following the scheme of point 1 , we conclude that in these beliefs regions there are the following possible PBE

(a) i. if $\gamma \in\left[\frac{1}{2}, 1\right]$

$$
\left\{d^{A *}\left(p^{A}, p^{B}\right), d^{B *}\left(p^{A}, p^{B}\right)\right\}= \begin{cases}\{0,0\} & \text { if }\left(p^{A}, p^{B}\right)=(1,1) \\ \{0,0\} & \text { if }\left(p^{A}, p^{B}\right)=(2,1) \\ \{0,0\} & \text { if }\left(p^{A}, p^{B}\right)=(1,2) \\ \{0,0\} & \text { if }\left(p^{A}, p^{B}\right)=(2,2)\end{cases}
$$

Now, we have to go back to terrorists' beliefs, considering pure strategy PBE only. Hence 
- when $\gamma \in\left[\frac{1}{2}, 1\right]$ then $\mu^{2,1 \mid 0,0}=\mu^{1,2 \mid 0,0}=(1-\pi) \pi$ which means that $\mu^{2,1 \mid 0,0} \leq \mu^{1,2 \mid 0,0}$ is satisfied.

10. Suppose

$$
\left\{\begin{array}{c}
\mu^{2,1 \mid 0,0} \geq \mu^{1,2 \mid 0,0} \\
\mu^{2,1 \mid 1,0} \leq \frac{15 \mu^{1,2 \mid 1,0}+10}{14} \\
\mu^{2,1 \mid 0,1} \geq \frac{14 \mu^{1,2 \mid 0,1}-10}{15} \\
\mu^{2,1 \mid 1,1} \leq \mu^{1,2 \mid 1,1}
\end{array}\right.
$$

Following the scheme of point 1 , we conclude that in these beliefs regions there are the following possible PBE

(a) i. if $\gamma \in\left[0, \frac{1}{12}\right]$

$$
\left\{d^{A *}\left(p^{A}, p^{B}\right), d^{B *}\left(p^{A}, p^{B}\right)\right\}= \begin{cases}\{1,1\} & \text { if }\left(p^{A}, p^{B}\right)=(1,1) \\ \{1,1\} & \text { if }\left(p^{A}, p^{B}\right)=(2,1) \\ \{1,1\} & \text { if }\left(p^{A}, p^{B}\right)=(1,2) \\ \{1,1\} & \text { if }\left(p^{A}, p^{B}\right)=(2,2)\end{cases}
$$

ii. if $\gamma \in\left[\frac{1}{12}, \frac{2}{15}\right]$

$$
\left\{d^{A *}\left(p^{A}, p^{B}\right), d^{B *}\left(p^{A}, p^{B}\right)\right\}= \begin{cases}\{1,1\} & \text { if }\left(p^{A}, p^{B}\right)=(1,1) \\ \{1,0\} & \text { if }\left(p^{A}, p^{B}\right)=(2,1) \\ \{1,1\} & \text { if }\left(p^{A}, p^{B}\right)=(1,2) \\ \{1,0\} & \text { if }\left(p^{A}, p^{B}\right)=(2,2)\end{cases}
$$

iii. if $\gamma \in\left[\frac{2}{15}, \frac{1}{6}\right]$

$$
\left\{d^{A *}\left(p^{A}, p^{B}\right), d^{B *}\left(p^{A}, p^{B}\right)\right\}= \begin{cases}\{1,1\} & \text { if }\left(p^{A}, p^{B}\right)=(1,1) \\ \{1,0\} & \text { if }\left(p^{A}, p^{B}\right)=(2,1) \\ \{1,0\} & \text { if }\left(p^{A}, p^{B}\right)=(1,2) \\ \{1,0\} & \text { if }\left(p^{A}, p^{B}\right)=(2,2)\end{cases}
$$

iv. if $\gamma \in\left[\frac{1}{6}, \frac{1}{4}\right]$

$$
\left\{d^{A *}\left(p^{A}, p^{B}\right), d^{B *}\left(p^{A}, p^{B}\right)\right\}= \begin{cases}\{1,0\} & \text { if }\left(p^{A}, p^{B}\right)=(1,1) \\ \{1,0\} & \text { if }\left(p^{A}, p^{B}\right)=(2,1) \\ \{1,0\} & \text { if }\left(p^{A}, p^{B}\right)=(1,2) \\ \{1,0\} & \text { if }\left(p^{A}, p^{B}\right)=(2,2)\end{cases}
$$

v. if $\gamma \in\left[\frac{1}{4}, \frac{1}{3}\right]$

$$
\left\{d^{A *}\left(p^{A}, p^{B}\right), d^{B *}\left(p^{A}, p^{B}\right)\right\}= \begin{cases}\{1,0\} & \text { if }\left(p^{A}, p^{B}\right)=(1,1) \\ \{1,0\} & \text { if }\left(p^{A}, p^{B}\right)=(2,1) \\ \{1,0\} & \text { if }\left(p^{A}, p^{B}\right)=(1,2) \\ \{0,0\} & \text { if }\left(p^{A}, p^{B}\right)=(2,2)\end{cases}
$$


vi. if $\gamma \in\left[\frac{1}{3}, \frac{1}{2}\right]$

$$
\left\{d^{A *}\left(p^{A}, p^{B}\right), d^{B *}\left(p^{A}, p^{B}\right)\right\}= \begin{cases}\{1,0\} & \text { if }\left(p^{A}, p^{B}\right)=(1,1) \\ \{0,0\} & \text { if }\left(p^{A}, p^{B}\right)=(2,1) \\ \{0,0\} & \text { if }\left(p^{A}, p^{B}\right)=(1,2) \\ \{0,0\} & \text { if }\left(p^{A}, p^{B}\right)=(2,2)\end{cases}
$$

vii. if $\gamma \in\left[\frac{1}{2}, 1\right]$

$$
\left\{d^{A *}\left(p^{A}, p^{B}\right), d^{B *}\left(p^{A}, p^{B}\right)\right\}= \begin{cases}\{0,0\} & \text { if }\left(p^{A}, p^{B}\right)=(1,1) \\ \{0,0\} & \text { if }\left(p^{A}, p^{B}\right)=(2,1) \\ \{0,0\} & \text { if }\left(p^{A}, p^{B}\right)=(1,2) \\ \{0,0\} & \text { if }\left(p^{A}, p^{B}\right)=(2,2)\end{cases}
$$

Now, we have to go back to terrorists' beliefs. Hence

- when $\gamma \in\left[0, \frac{1}{12}\right]$ then $\mu^{2,1 \mid 1,1}=\mu^{1,2 \mid 1,1}=(1-\pi) \pi$ which means that $\mu^{2,1 \mid 1,1} \leq \mu^{1,2 \mid 1,1}$ is satisfied.

- when $\gamma \in\left[\frac{1}{12}, \frac{2}{15}\right]$ then $\mu^{2,1 \mid 1,1}=0, \mu^{1,2 \mid 1,1}=\frac{(1-\pi) \pi}{\pi^{2}+(1-\pi) \pi}$ which means that $\mu^{2,1 \mid 1,1} \leq \mu^{1,2 \mid 1,1}$ is satisfied. Moreover $\mu^{2,1 \mid 1,0}=$ $\frac{(1-\pi) \pi}{(1-\pi) \pi+(1-\pi)^{2}}, \mu^{1,2 \mid 1,0}=0$ which means that $\mu^{2,1 \mid 1,0} \leq \frac{15 \mu^{1,2 \mid 1,0}+10}{14} \Leftrightarrow$ $\pi \leq \frac{4-\sqrt{16-12}}{6}=\frac{1}{3} \vee \pi \geq \frac{4+\sqrt{16-12}}{6}=1$

- when $\gamma \in\left[\frac{2}{15}, \frac{1}{6}\right]$ then $\mu^{2,1 \mid 1,1}=\mu^{1,2 \mid 1,1}=0$ which means that $\mu^{2,1 \mid 1,1} \leq \mu^{1,2 \mid 1,1}$ is satisfied. Moreover $\mu^{2,1 \mid 1,0}=\mu^{1,2 \mid 1,0}=$ $\frac{(1-\pi) \pi}{2(1-\pi) \pi+(1-\pi)^{2}}$ which means that $\mu^{2,1 \mid 1,0} \leq \frac{15 \mu^{1,2 \mid 1,0}+10}{14}$ is satisfied.

- when $\gamma \in\left[\frac{1}{6}, \frac{1}{4}\right]$ then $\mu^{2,1 \mid 1,0}=\mu^{1,2 \mid 1,0}=(1-\pi) \pi$ which means that $\mu^{2,1 \mid 1,0} \leq \frac{15 \mu^{1,2 \mid 1,0}+10}{14}$ is satisfied.

- when $\gamma \in\left[\frac{1}{4}, \frac{1}{3}\right]$ then $\mu^{2,1 \mid 0,0}=\mu^{1,2 \mid 0,0}=0$ which means that $\mu^{2,1 \mid 0,0} \geq \mu^{1,2 \mid 0,0}$ is satisfied. Moreover $\mu^{2,1 \mid 1,0}=\mu^{1,2 \mid 1,0}=$ $\frac{(1-\pi) \pi}{2(1-\pi) \pi+\pi^{2}}$ which means that $\mu^{2,1 \mid 1,0} \leq \frac{15 \mu^{1,2 \mid 1,0}+10}{14}$ is satisfied.

- when $\gamma \in\left[\frac{1}{3}, \frac{1}{2}\right]$ then $\mu^{2,1 \mid 0,0}=\mu^{1,2 \mid 0,0}=\frac{(1-\pi) \pi}{2(1-\pi) \pi+(1-\pi)^{2}} \quad$ which means that $\mu^{2,1 \mid 0,0} \geq \mu^{1,2 \mid 0,0}$ is satisfied. Moreover $\mu^{2,1 \mid 1,0}=\mu^{1,2 \mid 1,0}=$ 0 which means that $\mu^{2,1 \mid 1,0} \leq \frac{15 \mu^{1,2 \mid 1,0}+10}{14}$ is satisfied.

- when $\gamma \in\left[\frac{1}{2}, 1\right]$ then $\mu^{2,1 \mid 0,0}=\mu^{1,2 \mid 0,0}=(1-\pi) \pi$ which means that $\mu^{2,1 \mid 0,0} \geq \mu^{1,2 \mid 0,0}$ is satisfied.

11. Suppose

$$
\left\{\begin{aligned}
& \mu^{2,1 \mid 0,0} \geq \mu^{1,2 \mid 0,0} \\
& \mu^{2,1 \mid 1,0} \geq \frac{15 \mu^{1,2 \mid 1,0}+10}{14} \\
& \mu^{2,1 \mid 0,1} \leq \frac{14 \mu^{1,2 \mid 0,1}-10}{15} \\
& \mu^{2,1 \mid 1,1} \leq \mu^{1,2 \mid 1,1}
\end{aligned}\right.
$$


Following the scheme of point 1 , we conclude that in these beliefs regions there are the following possible $\mathrm{PBE}$

(a) i. if $\gamma \in\left[0, \frac{1}{12}\right]$

$$
\left\{d^{A *}\left(p^{A}, p^{B}\right), d^{B *}\left(p^{A}, p^{B}\right)\right\}= \begin{cases}\{1,0\} & \text { if }\left(p^{A}, p^{B}\right)=(1,1) \\ \{1,0\} & \text { if }\left(p^{A}, p^{B}\right)=(2,1) \\ \{1,0\} & \text { if }\left(p^{A}, p^{B}\right)=(1,2) \\ \{1,0\} & \text { if }\left(p^{A}, p^{B}\right)=(2,2)\end{cases}
$$

ii. if $\gamma \in\left[\frac{1}{12}, \frac{2}{15}\right]$

$$
\left\{d^{A *}\left(p^{A}, p^{B}\right), d^{B *}\left(p^{A}, p^{B}\right)\right\}= \begin{cases}\{1,0\} & \text { if }\left(p^{A}, p^{B}\right)=(1,1) \\ \{1,0\} & \text { if }\left(p^{A}, p^{B}\right)=(2,1) \\ \{0,0\} & \text { if }\left(p^{A}, p^{B}\right)=(1,2) \\ \{0,0\} & \text { if }\left(p^{A}, p^{B}\right)=(2,2)\end{cases}
$$

iii. if $\gamma \in\left[\frac{2}{15}, \frac{1}{6}\right]$

$$
\left\{d^{A *}\left(p^{A}, p^{B}\right), d^{B *}\left(p^{A}, p^{B}\right)\right\}= \begin{cases}\{1,0\} & \text { if }\left(p^{A}, p^{B}\right)=(1,1) \\ \{0,0\} & \text { if }\left(p^{A}, p^{B}\right)=(2,1) \\ \{0,0\} & \text { if }\left(p^{A}, p^{B}\right)=(1,2) \\ \{0,0\} & \text { if }\left(p^{A}, p^{B}\right)=(2,2)\end{cases}
$$

iv. if $\gamma \in\left[\frac{1}{6}, 1\right]$

$$
\left\{d^{A *}\left(p^{A}, p^{B}\right), d^{B *}\left(p^{A}, p^{B}\right)\right\}= \begin{cases}\{0,0\} & \text { if }\left(p^{A}, p^{B}\right)=(1,1) \\ \{0,0\} & \text { if }\left(p^{A}, p^{B}\right)=(2,1) \\ \{0,0\} & \text { if }\left(p^{A}, p^{B}\right)=(1,2) \\ \{0,0\} & \text { if }\left(p^{A}, p^{B}\right)=(2,2)\end{cases}
$$

Now, we have to go back to terrorists' beliefs. Hence

- when $\gamma \in\left[0, \frac{1}{12}\right]$ then $\mu^{2,1 \mid 1,0}=\mu^{1,2 \mid 1,0}=(1-\pi) \pi$ which means that $\mu^{2,1 \mid 1,0} \geq \frac{15 \mu^{1,2 \mid 1,0}+10}{14}$ is not satisfied.

- when $\gamma \in\left[\frac{1}{12}, \frac{2}{15}\right]$ then $\mu^{2,1 \mid 0,0}=0, \mu^{1,2 \mid 0,0}=\frac{(1-\pi) \pi}{(1-\pi) \pi+(1-\pi)^{2}}$ which means that $\mu^{2,1 \mid 0,0} \geq \mu^{1,2 \mid 0,0}$ is not satisfied

- when $\gamma \in\left[\frac{2}{15}, \frac{1}{6}\right]$ then $\mu^{2,1 \mid 1,0}=\mu^{1,2 \mid 1,0}=0$ which means that $\mu^{2,1 \mid 1,0} \geq \frac{15 \mu^{1,2 \mid 1,0}+10}{14}$ is not satisfied.

- when $\gamma \in\left[\frac{1}{6}, 1\right]$ then $\mu^{2,1 \mid 0,0}=\mu^{1,2 \mid 0,0}=(1-\pi) \pi$ which means that $\mu^{2,1 \mid 0,0} \geq \mu^{1,2 \mid 0,0}$ is satisfied.

12. Suppose

$$
\left\{\begin{array}{c}
\mu^{2,1 \mid 0,0} \leq \mu^{1,2 \mid 0,0} \\
\mu^{2,1 \mid 1,0} \leq \frac{15 \mu^{1,2 \mid 1,0}+10}{14} \\
\mu^{2,1 \mid 0,1} \leq \frac{14 \mu^{1,2 \mid 0,1}-10}{15} \\
\mu^{2,1 \mid 1,1} \geq \mu^{1,2 \mid 1,1}
\end{array}\right.
$$


Following the scheme of point 1 , we conclude that in these beliefs regions there are the following possible $\mathrm{PBE}$

(a) i. if $\gamma \in\left[0, \frac{1}{12}\right]$

$$
\left\{d^{A *}\left(p^{A}, p^{B}\right), d^{B *}\left(p^{A}, p^{B}\right)\right\}= \begin{cases}\{0,1\} & \text { if }\left(p^{A}, p^{B}\right)=(1,1) \\ \{0,1\} & \text { if }\left(p^{A}, p^{B}\right)=(2,1) \\ \{0,1\} & \text { if }\left(p^{A}, p^{B}\right)=(1,2) \\ \{0,1\} & \text { if }\left(p^{A}, p^{B}\right)=(2,2)\end{cases}
$$

ii. if $\gamma \in\left[\frac{1}{12}, \frac{2}{15}\right]$

$$
\left\{d^{A *}\left(p^{A}, p^{B}\right), d^{B *}\left(p^{A}, p^{B}\right)\right\}= \begin{cases}\{0,1\} & \text { if }\left(p^{A}, p^{B}\right)=(1,1) \\ \{0,0\} & \text { if }\left(p^{A}, p^{B}\right)=(2,1) \\ \{0,1\} & \text { if }\left(p^{A}, p^{B}\right)=(1,2) \\ \{0,0\} & \text { if }\left(p^{A}, p^{B}\right)=(2,2)\end{cases}
$$

iii. if $\gamma \in\left[\frac{2}{15}, \frac{1}{6}\right]$

$$
\left\{d^{A *}\left(p^{A}, p^{B}\right), d^{B *}\left(p^{A}, p^{B}\right)\right\}= \begin{cases}\{0,1\} & \text { if }\left(p^{A}, p^{B}\right)=(1,1) \\ \{0,0\} & \text { if }\left(p^{A}, p^{B}\right)=(2,1) \\ \{0,0\} & \text { if }\left(p^{A}, p^{B}\right)=(1,2) \\ \{0,0\} & \text { if }\left(p^{A}, p^{B}\right)=(2,2)\end{cases}
$$

iv. if $\gamma \in\left[\frac{1}{6}, 1\right]$

$$
\left\{d^{A *}\left(p^{A}, p^{B}\right), d^{B *}\left(p^{A}, p^{B}\right)\right\}= \begin{cases}\{0,0\} & \text { if }\left(p^{A}, p^{B}\right)=(1,1) \\ \{0,0\} & \text { if }\left(p^{A}, p^{B}\right)=(2,1) \\ \{0,0\} & \text { if }\left(p^{A}, p^{B}\right)=(1,2) \\ \{0,0\} & \text { if }\left(p^{A}, p^{B}\right)=(2,2)\end{cases}
$$

Now, we have to go back to terrorists' beliefs. Hence

- when $\gamma \in\left[0, \frac{1}{12}\right]$ then $\mu^{2,1 \mid 0,1}=\mu^{1,2 \mid 0,1}=(1-\pi) \pi$ which means that $\mu^{2,1 \mid 0,1} \leq \frac{14 \mu^{1,2 \mid 0,1}-10}{15}$ is not satisfied.

- when $\gamma \in\left[\frac{1}{12}, \frac{2}{15}\right]$ then $\mu^{2,1 \mid 0,0}=\frac{(1-\pi) \pi}{(1-\pi) \pi+(1-\pi)^{2}}, \mu^{1,2 \mid 0,0}=0$ which means that $\mu^{2,1 \mid 0,0} \leq \mu^{1,2 \mid 0,0}$ is not satisfied

- when $\gamma \in\left[\frac{2}{15}, \frac{1}{6}\right]$ then $\mu^{2,1 \mid 0,1}=\mu^{1,2 \mid 0,1}=0$ which means that $\mu^{2,1 \mid 0,1} \leq \frac{15 \mu^{1,2 \mid 0,1}-10}{14}$ is not satisfied.

- when $\gamma \in\left[\frac{1}{6}, 1\right]$ then $\mu^{2,1 \mid 0,0}=\mu^{1,2 \mid 0,0}=(1-\pi) \pi$ which means that $\mu^{2,1 \mid 0,0} \leq \mu^{1,2 \mid 0,0}$ is satisfied.

13. Suppose

$$
\left\{\begin{array}{c}
\mu^{2,1 \mid 0,0} \geq \mu^{1,2 \mid 0,0} \\
\mu^{2,1 \mid 1,0} \leq \frac{15 \mu^{1,2 \mid 1,0}+10}{14} \\
\mu^{2,1 \mid 0,1} \leq \frac{14 \mu^{1,2 \mid 0,1}-10}{15} \\
\mu^{2,1 \mid 1,1} \leq \mu^{1,2 \mid 1,1}
\end{array}\right.
$$


Following the scheme of point 1 , we conclude that in these beliefs regions there are the following possible $\mathrm{PBE}$

(a) i. if $\gamma \in\left[\frac{1}{6}, \frac{1}{4}\right]$

$$
\left\{d^{A *}\left(p^{A}, p^{B}\right), d^{B *}\left(p^{A}, p^{B}\right)\right\}= \begin{cases}\{1,0\} & \text { if }\left(p^{A}, p^{B}\right)=(1,1) \\ \{1,0\} & \text { if }\left(p^{A}, p^{B}\right)=(2,1) \\ \{1,0\} & \text { if }\left(p^{A}, p^{B}\right)=(1,2) \\ \{1,0\} & \text { if }\left(p^{A}, p^{B}\right)=(2,2)\end{cases}
$$

ii. if $\gamma \in\left[\frac{1}{4}, \frac{1}{3}\right]$

$$
\left\{d^{A *}\left(p^{A}, p^{B}\right), d^{B *}\left(p^{A}, p^{B}\right)\right\}= \begin{cases}\{1,0\} & \text { if }\left(p^{A}, p^{B}\right)=(1,1) \\ \{1,0\} & \text { if }\left(p^{A}, p^{B}\right)=(2,1) \\ \{1,0\} & \text { if }\left(p^{A}, p^{B}\right)=(1,2) \\ \{0,0\} & \text { if }\left(p^{A}, p^{B}\right)=(2,2)\end{cases}
$$

iii. if $\gamma \in\left[\frac{1}{3}, \frac{1}{2}\right]$

$$
\left\{d^{A *}\left(p^{A}, p^{B}\right), d^{B *}\left(p^{A}, p^{B}\right)\right\}= \begin{cases}\{1,0\} & \text { if }\left(p^{A}, p^{B}\right)=(1,1) \\ \{0,0\} & \text { if }\left(p^{A}, p^{B}\right)=(2,1) \\ \{0,0\} & \text { if }\left(p^{A}, p^{B}\right)=(1,2) \\ \{0,0\} & \text { if }\left(p^{A}, p^{B}\right)=(2,2)\end{cases}
$$

iv. if $\gamma \in\left[\frac{1}{2}, 1\right]$

$$
\left\{d^{A *}\left(p^{A}, p^{B}\right), d^{B *}\left(p^{A}, p^{B}\right)\right\}= \begin{cases}\{0,0\} & \text { if }\left(p^{A}, p^{B}\right)=(1,1) \\ \{0,0\} & \text { if }\left(p^{A}, p^{B}\right)=(2,1) \\ \{0,0\} & \text { if }\left(p^{A}, p^{B}\right)=(1,2) \\ \{0,0\} & \text { if }\left(p^{A}, p^{B}\right)=(2,2)\end{cases}
$$

Now, we have to go back to terrorists' beliefs. Hence

- when $\gamma \in\left[\frac{1}{6}, \frac{1}{4}\right]$ then $\mu^{2,1 \mid 1,0}=\mu^{1,2 \mid 1,0}=(1-\pi) \pi$ which means that $\mu^{2,1 \mid 1,0} \leq \frac{15 \mu^{1,2 \mid 1,0}+10}{14}$ is satisfied.

- when $\gamma \in\left[\frac{1}{4}, \frac{1}{3}\right]$ then $\mu^{2,1 \mid 1,0}=\mu^{1,2 \mid 1,0}=\frac{(1-\pi) \pi}{\pi^{2}+2(1-\pi) \pi}$ which means that $\mu^{2,1 \mid 1,0} \leq \frac{15 \mu^{1,2 \mid 1,0}+10}{14}$ is satisfied. Moreover $\mu^{2,1 \mid 0,0}=\mu^{1,2 \mid 0,0}=$ 0 which means that $\mu^{2,1 \mid 0,0} \geq \mu^{1,2 \mid 0,0}$ is satisfied

- when $\gamma \in\left[\frac{1}{3}, \frac{1}{2}\right]$ then $\mu^{2,1 \mid 1,0}=\mu^{1,2 \mid 1,0}=0$ which means that $\mu^{2,1 \mid 1,0} \leq \frac{15 \mu^{1,2 \mid 1,0}+10}{14}$ is satisfied. Moreover $\mu^{2,1 \mid 0,0}=\mu^{1,2 \mid 0,0}=$ $\frac{(1-\pi) \pi}{2(1-\pi) \pi+(1-\pi)^{2}}$ which means that $\mu^{2,1 \mid 0,0} \geq \mu^{1,2 \mid 0,0}$ is satisfied

- when $\gamma \in\left[\frac{1}{2}, 1\right]$ then $\mu^{2,1 \mid 0,0}=\mu^{1,2 \mid 0,0}=(1-\pi) \pi$ which means that $\mu^{2,1 \mid 0,0} \geq \mu^{1,2 \mid 0,0}$ is satisfied. 
14. Suppose

$$
\left\{\begin{array}{c}
\mu^{2,1 \mid 0,0} \leq \mu^{1,2 \mid 0,0} \\
\mu^{2,1 \mid 1,0} \geq \frac{15 \mu^{1,2 \mid 1,0}+10}{14} \\
\mu^{2,1 \mid 0,1} \leq \frac{14 \mu^{1,2 \mid 0,1}-10}{15} \\
\mu^{2,1 \mid 1,1} \leq \mu^{1,2 \mid 1,1}
\end{array}\right.
$$

Following the scheme of point 1 , we conclude that in these beliefs regions there are the following possible PBE

(a) i. if $\gamma \in\left[0, \frac{1}{12}\right]$

$$
\left\{d^{A *}\left(p^{A}, p^{B}\right), d^{B *}\left(p^{A}, p^{B}\right)\right\}= \begin{cases}\{0,1\} & \text { if }\left(p^{A}, p^{B}\right)=(1,1) \\ \{0,1\} & \text { if }\left(p^{A}, p^{B}\right)=(2,1) \\ \{0,1\} & \text { if }\left(p^{A}, p^{B}\right)=(1,2) \\ \{0,1\} & \text { if }\left(p^{A}, p^{B}\right)=(2,2)\end{cases}
$$

ii. if $\gamma \in\left[\frac{1}{12}, \frac{2}{15}\right]$

$$
\left\{d^{A *}\left(p^{A}, p^{B}\right), d^{B *}\left(p^{A}, p^{B}\right)\right\}= \begin{cases}\{0,1\} & \text { if }\left(p^{A}, p^{B}\right)=(1,1) \\ \{0,0\} & \text { if }\left(p^{A}, p^{B}\right)=(2,1) \\ \{0,1\} & \text { if }\left(p^{A}, p^{B}\right)=(1,2) \\ \{0,0\} & \text { if }\left(p^{A}, p^{B}\right)=(2,2)\end{cases}
$$

iii. if $\gamma \in\left[\frac{2}{15}, \frac{1}{6}\right]$

$$
\left\{d^{A *}\left(p^{A}, p^{B}\right), d^{B *}\left(p^{A}, p^{B}\right)\right\}= \begin{cases}\{0,1\} & \text { if }\left(p^{A}, p^{B}\right)=(1,1) \\ \{0,0\} & \text { if }\left(p^{A}, p^{B}\right)=(2,1) \\ \{0,0\} & \text { if }\left(p^{A}, p^{B}\right)=(1,2) \\ \{0,0\} & \text { if }\left(p^{A}, p^{B}\right)=(2,2)\end{cases}
$$

iv. if $\gamma \in\left[\frac{1}{6}, 1\right]$

$$
\left\{d^{A *}\left(p^{A}, p^{B}\right), d^{B *}\left(p^{A}, p^{B}\right)\right\}= \begin{cases}\{0,0\} & \text { if }\left(p^{A}, p^{B}\right)=(1,1) \\ \{0,0\} & \text { if }\left(p^{A}, p^{B}\right)=(2,1) \\ \{0,0\} & \text { if }\left(p^{A}, p^{B}\right)=(1,2) \\ \{0,0\} & \text { if }\left(p^{A}, p^{B}\right)=(2,2)\end{cases}
$$

Now, we have to go back to terrorists' beliefs. Hence

- when $\gamma \in\left[0, \frac{1}{12}\right]$ then $\mu^{2,1 \mid 0,1}=\mu^{1,2 \mid 0,1}=(1-\pi) \pi$ which means that $\mu^{2,1 \mid 0,1} \leq \frac{14 \mu^{1,2 \mid 0,1}-10}{15}$ is not satisfied.

- when $\gamma \in\left[\frac{1}{12}, \frac{2}{15}\right]$ then $\mu^{2,1 \mid 0,0}=\frac{(1-\pi) \pi}{(1-\pi) \pi+(1-\pi)^{2}}, \mu^{1,2 \mid 0,0}=0$ which means that $\mu^{2,1 \mid 0,0} \leq \mu^{1,2 \mid 0,0}$ is not satisfied

- when $\gamma \in\left[\frac{2}{15}, \frac{1}{6}\right]$ then $\mu^{2,1 \mid 0,1}=\mu^{1,2 \mid 0,1}=0$ which means that $\mu^{2,1 \mid 0,1} \leq \frac{14 \mu^{1,2 \mid 0,1}-10}{15}$ is not satisfied. 
- when $\gamma \in\left[\frac{1}{6}, 1\right]$ then $\mu^{2,1 \mid 0,0}=\mu^{1,2 \mid 0,0}=(1-\pi) \pi$ which means that $\mu^{2,1 \mid 0,0} \leq \mu^{1,2 \mid 0,0}$ is satisfied.

15. Suppose

$$
\left\{\begin{array}{c}
\mu^{2,1 \mid 0,0} \leq \mu^{1,2 \mid 0,0} \\
\mu^{2,1 \mid 1,0} \leq \frac{15 \mu^{1,2 \mid 1,0}+10}{14} \\
\mu^{2,1 \mid 0,1} \geq \frac{14 \mu^{1,2 \mid 0,1}-10}{15} \\
\mu^{2,1 \mid 1,1} \leq \mu^{1,2 \mid 1,1}
\end{array}\right.
$$

Following the scheme of point 1 , we conclude that in these beliefs regions there are the following possible $\mathrm{PBE}$

(a) i. if $\gamma \in\left[0, \frac{1}{12}\right]$

$$
\left\{d^{A *}\left(p^{A}, p^{B}\right), d^{B *}\left(p^{A}, p^{B}\right)\right\}= \begin{cases}\{1,1\} & \text { if }\left(p^{A}, p^{B}\right)=(1,1) \\ \{1,1\} & \text { if }\left(p^{A}, p^{B}\right)=(2,1) \\ \{1,1\} & \text { if }\left(p^{A}, p^{B}\right)=(1,2) \\ \{1,1\} & \text { if }\left(p^{A}, p^{B}\right)=(2,2)\end{cases}
$$

ii. if $\gamma \in\left[\frac{1}{2}, 1\right]$

$$
\left\{d^{A *}\left(p^{A}, p^{B}\right), d^{B *}\left(p^{A}, p^{B}\right)\right\}= \begin{cases}\{0,0\} & \text { if }\left(p^{A}, p^{B}\right)=(1,1) \\ \{0,0\} & \text { if }\left(p^{A}, p^{B}\right)=(2,1) \\ \{0,0\} & \text { if }\left(p^{A}, p^{B}\right)=(1,2) \\ \{0,0\} & \text { if }\left(p^{A}, p^{B}\right)=(2,2)\end{cases}
$$

Now, we have to go back to terrorists' beliefs. Hence

- when $\gamma \in\left[0, \frac{1}{12}\right]$ then $\mu^{2,1 \mid 1,1}=\mu^{1,2 \mid 1,1}=(1-\pi) \pi$ which means that $\mu^{2,1 \mid 1,1} \leq \mu^{1,2 \mid 1,1}$ is satisfied.

- when $\gamma \in\left[\frac{1}{2}, 1\right]$ then $\mu^{2,1 \mid 0,0}=\mu^{1,2 \mid 0,0}=(1-\pi) \pi$ which means that $\mu^{2,1 \mid 0,0} \leq \mu^{1,2 \mid 0,0}$ is satisfied.

16. Suppose

$$
\left\{\begin{array}{c}
\mu^{2,1 \mid 0,0} \leq \mu^{1,2 \mid 0,0} \\
\mu^{2,1 \mid 1,0} \leq \frac{15 \mu^{1,2 \mid 1,0}+10}{14} \\
\mu^{2,1 \mid 0,1} \leq \frac{14 \mu^{1,2 \mid 0,1}-10}{15} \\
\mu^{2,1 \mid 1,1} \leq \mu^{1,2 \mid 1,1}
\end{array}\right.
$$

Following the scheme of point 1 , we conclude that in these beliefs regions there are the following possible PBE

(a) i. if $\gamma \in\left[0, \frac{1}{12}\right]$

$$
\left\{d^{A *}\left(p^{A}, p^{B}\right), d^{B *}\left(p^{A}, p^{B}\right)\right\}= \begin{cases}\{0,1\} & \text { if }\left(p^{A}, p^{B}\right)=(1,1) \\ \{0,1\} & \text { if }\left(p^{A}, p^{B}\right)=(2,1) \\ \{0,1\} & \text { if }\left(p^{A}, p^{B}\right)=(1,2) \\ \{0,1\} & \text { if }\left(p^{A}, p^{B}\right)=(2,2)\end{cases}
$$


ii. if $\gamma \in\left[\frac{1}{12}, \frac{2}{15}\right]$

$$
\left\{d^{A *}\left(p^{A}, p^{B}\right), d^{B *}\left(p^{A}, p^{B}\right)\right\}= \begin{cases}\{0,1\} & \text { if }\left(p^{A}, p^{B}\right)=(1,1) \\ \{0,0\} & \text { if }\left(p^{A}, p^{B}\right)=(2,1) \\ \{0,1\} & \text { if }\left(p^{A}, p^{B}\right)=(1,2) \\ \{0,0\} & \text { if }\left(p^{A}, p^{B}\right)=(2,2)\end{cases}
$$

iii. if $\gamma \in\left[\frac{2}{15}, \frac{1}{6}\right]$

$$
\left\{d^{A *}\left(p^{A}, p^{B}\right), d^{B *}\left(p^{A}, p^{B}\right)\right\}= \begin{cases}\{0,1\} & \text { if }\left(p^{A}, p^{B}\right)=(1,1) \\ \{0,0\} & \text { if }\left(p^{A}, p^{B}\right)=(2,1) \\ \{0,0\} & \text { if }\left(p^{A}, p^{B}\right)=(1,2) \\ \{0,0\} & \text { if }\left(p^{A}, p^{B}\right)=(2,2)\end{cases}
$$

iv. if $\gamma \in\left[\frac{1}{6}, 1\right]$

$$
\left\{d^{A *}\left(p^{A}, p^{B}\right), d^{B *}\left(p^{A}, p^{B}\right)\right\}= \begin{cases}\{0,0\} & \text { if } \quad\left(p^{A}, p^{B}\right)=(1,1) \\ \{0,0\} & \text { if } \quad\left(p^{A}, p^{B}\right)=(2,1) \\ \{0,0\} & \text { if }\left(p^{A}, p^{B}\right)=(1,2) \\ \{0,0\} & \text { if }\left(p^{A}, p^{B}\right)=(2,2)\end{cases}
$$

Now, we have to go back to terrorists' beliefs. Hence

- when $\gamma \in\left[0, \frac{1}{12}\right]$ then $\mu^{2,1 \mid 0,1}=\mu^{1,2 \mid 0,1}=(1-\pi) \pi$ which means that $\mu^{2,1 \mid 0,1} \leq \frac{14 \mu^{1,2 \mid 0,1}-10}{15}$ is not satisfied.

- when $\gamma \in\left[\frac{1}{12}, \frac{2}{15}\right]$ then $\mu^{2,1 \mid 0,0}=\frac{(1-\pi) \pi}{(1-\pi) \pi+(1-\pi)^{2}}, \mu^{1,2 \mid 0,0}=0$ which means that $\mu^{2,1 \mid 0,0} \leq \mu^{1,2 \mid 0,0}$ is not satisfied.

- when $\gamma \in\left[\frac{2}{15}, \frac{1}{6}\right]$ then $\mu^{2,1 \mid 0,1}=\mu^{1,2 \mid 0,1}=0$ which means that $\mu^{2,1 \mid 0,1} \leq \frac{14 \mu^{1,2 \mid 0,1}-10}{15}$ is not satisfied.

- when $\gamma \in\left[\frac{1}{6}, 1\right]$ then $\mu^{2,1 \mid 0,0}=\mu^{1,2 \mid 0,0}=(1-\pi) \pi$ which means that $\mu^{2,1 \mid 0,0} \leq \mu^{1,2 \mid 0,0}$ is satisfied.

Considering the 16 possible combination of beliefs and the consequent sequential rational choice of the countries we are able to characterize the entire set of equilibria.

Proposition 7 When $\gamma \in\left[\frac{1}{6}, 1\right]$ there are multiple PBE such that

1 .

$$
\left\{d^{A *}\left(p^{A}, p^{B}\right), d^{B *}\left(p^{A}, p^{B}\right)\right\}= \begin{cases}\{0,0\} & \text { if }\left(p^{A}, p^{B}\right)=(1,1) \\ \{0,0\} & \text { if }\left(p^{A}, p^{B}\right)=(2,1) \\ \{0,0\} & \text { if }\left(p^{A}, p^{B}\right)=(1,2) \\ \{0,0\} & \text { if }\left(p^{A}, p^{B}\right)=(2,2)\end{cases}
$$




$$
\begin{aligned}
& \left\{\begin{array}{c}
\mu^{2,1 \mid 0,0}=\mu^{1,2 \mid 0,0}=(1-\pi) \pi \\
\mu^{2,1 \mid 1,0} \geq \frac{15 \mu^{1,2 \mid 1,0}+10}{14} \\
\mu^{2,1 \mid 0,1} \geq \frac{14 \mu^{1,2 \mid 0,1}-10}{15} \\
\mu^{2,1 \mid 1,1} \geq \mu^{1,2 \mid 1,1}
\end{array}\right. \\
& a^{*}\left(d^{A}, d^{B}\right) \in \begin{cases}\{1\} & \left(d^{A}, d^{B}\right)=(0,0) \\
\{1\} & \left(d^{A}, d^{B}\right)=(1,0) \\
\{1\} & \left(d^{A}, d^{B}\right)=(0,1) \\
\{1\} & \left(d^{A}, d^{B}\right)=(1,1)\end{cases}
\end{aligned}
$$

2.

$$
\begin{aligned}
& \left\{d^{A *}\left(p^{A}, p^{B}\right), d^{B *}\left(p^{A}, p^{B}\right)\right\}= \begin{cases}\{0,0\} & \text { if }\left(p^{A}, p^{B}\right)=(1,1) \\
\{0,0\} & \text { if }\left(p^{A}, p^{B}\right)=(2,1) \\
\{0,0\} & \text { if }\left(p^{A}, p^{B}\right)=(1,2) \\
\{0,0\} & \text { if }\left(p^{A}, p^{B}\right)=(2,2)\end{cases} \\
& \left\{\begin{array}{c}
\mu^{2,1 \mid 0,0}=\mu^{1,2 \mid 0,0}=(1-\pi) \pi \\
\mu^{2,1 \mid 1,0} \geq \frac{15 \mu^{1,2 \mid 1,0}+10}{14} \\
\mu^{2,1 \mid 0,1} \geq \frac{14 \mu^{1,2 \mid 0,1}-10}{15} \\
\mu^{2,1 \mid 1,1} \leq \mu^{1,2 \mid 1,1}
\end{array}\right. \\
& a^{*}\left(d^{A}, d^{B}\right) \in \begin{cases}\{1\} & \left(d^{A}, d^{B}\right)=(0,0) \\
\{1\} & \left(d^{A}, d^{B}\right)=(1,0) \\
\{1\} & \left(d^{A}, d^{B}\right)=(0,1) \\
\{0\} & \left(d^{A}, d^{B}\right)=(1,1)\end{cases}
\end{aligned}
$$

3.

$$
\begin{gathered}
\left\{d^{A *}\left(p^{A}, p^{B}\right), d^{B *}\left(p^{A}, p^{B}\right)\right\}=\left\{\begin{array}{l}
\{0,0\} \quad \text { if }\left(p^{A}, p^{B}\right)=(1,1) \\
\{0,0\} \quad \text { if }\left(p^{A}, p^{B}\right)=(2,1) \\
\{0,0\} \quad \text { if }\left(p^{A}, p^{B}\right)=(1,2) \\
\{0,0\} \quad \text { if }\left(p^{A}, p^{B}\right)=(2,2)
\end{array}\right. \\
\left\{\begin{array}{r}
\mu^{2,1 \mid 0,0}=\mu^{1,2 \mid 0,0}=(1-\pi) \pi \\
\mu^{2,1 \mid 1,0} \geq \frac{15 \mu^{1,2 \mid 1,0}+10}{14} \\
\mu^{2,1 \mid 0,1} \leq \frac{14 \mu^{1,2 \mid 0,1}-10}{15} \\
\mu^{2,1 \mid 1,1} \geq \mu^{1,2 \mid 1,1}
\end{array}\right. \\
a^{*}\left(d^{A}, d^{B}\right) \in\left\{\begin{array}{r}
\{1\} \quad\left(d^{A}, d^{B}\right)=(0,0) \\
\{1\} \quad\left(d^{A}, d^{B}\right)=(1,0) \\
\{0\} \quad\left(d^{A}, d^{B}\right)=(0,1) \\
\{1\} \quad\left(d^{A}, d^{B}\right)=(1,1)
\end{array}\right.
\end{gathered}
$$

4.

$$
\left\{d^{A *}\left(p^{A}, p^{B}\right), d^{B *}\left(p^{A}, p^{B}\right)\right\} \in \begin{cases}\{(0,0)\} & \text { if }\left(p^{A}, p^{B}\right)=(1,1) \\ \{(0,0)\} & \text { if }\left(p^{A}, p^{B}\right)=(2,1) \\ \{(0,0)\} & \text { if }\left(p^{A}, p^{B}\right)=(1,2) \\ \{(0,0)\} & \text { if }\left(p^{A}, p^{B}\right)=(2,2)\end{cases}
$$




$$
\begin{aligned}
& \left\{\begin{array}{c}
\mu^{2,1 \mid 0,0}=\mu^{1,2 \mid 0,0}=(1-\pi) \pi \\
\mu^{2,1 \mid 1,0} \leq \frac{15 \mu^{1,2 \mid 1,0}+10}{14} \\
\mu^{2,1 \mid 0,1} \geq \frac{14 \mu^{1,2 \mid 0,1}-10}{15} \\
\mu^{2,1 \mid 1,1} \geq \mu^{1,2 \mid 1,1}
\end{array}\right. \\
& a^{*}\left(d^{A}, d^{B}\right) \in \begin{cases}\{1\} & \left(d^{A}, d^{B}\right)=(0,0) \\
\{0\} & \left(d^{A}, d^{B}\right)=(1,0) \\
\{1\} & \left(d^{A}, d^{B}\right)=(0,1) \\
\{1\} & \left(d^{A}, d^{B}\right)=(1,1)\end{cases}
\end{aligned}
$$

5.

$$
\begin{gathered}
\left\{d^{A *}\left(p^{A}, p^{B}\right), d^{B *}\left(p^{A}, p^{B}\right)\right\}=\left\{\begin{array}{l}
\{(0,0)\} \quad \text { if }\left(p^{A}, p^{B}\right)=(1,1) \\
\{(0,0)\} \quad \text { if }\left(p^{A}, p^{B}\right)=(2,1) \\
\{(0,0)\} \quad \text { if }\left(p^{A}, p^{B}\right)=(1,2) \\
\{(0,0)\} \quad \text { if }\left(p^{A}, p^{B}\right)=(2,2)
\end{array}\right. \\
\left\{\begin{array}{r}
\mu^{2,1 \mid 0,0}=\mu^{1,2 \mid 0,0}=(1-\pi) \pi \\
\mu^{2,1 \mid 1,0} \geq \frac{15 \mu^{1,2 \mid 1,0}+10}{14} \\
\mu^{2,1 \mid 0,1} \geq \frac{14 \mu^{1,2 \mid 0,1}-10}{15}
\end{array}\right. \\
\mu^{2,1 \mid 1,1} \geq \mu^{1,2 \mid 1,1} \\
a^{*}\left(d^{A}, d^{B}\right) \in\left\{\begin{array}{r}
\{0\} \quad\left(d^{A}, d^{B}\right)=(0,0) \\
\{1\} \quad\left(d^{A}, d^{B}\right)=(1,0) \\
\{1\} \quad\left(d^{A}, d^{B}\right)=(0,1) \\
\{1\} \quad\left(d^{A}, d^{B}\right)=(1,1)
\end{array}\right.
\end{gathered}
$$

6.

$$
\begin{gathered}
\left\{d^{A *}\left(p^{A}, p^{B}\right), d^{B *}\left(p^{A}, p^{B}\right)\right\}=\left\{\begin{array}{l}
\{0,0\} \quad \text { if }\left(p^{A}, p^{B}\right)=(1,1) \\
\{0,0\} \quad \text { if }\left(p^{A}, p^{B}\right)=(2,1) \\
\{0,0\} \quad \text { if }\left(p^{A}, p^{B}\right)=(1,2) \\
\{0,0\} \quad \text { if }\left(p^{A}, p^{B}\right)=(2,2)
\end{array}\right. \\
\left\{\begin{array}{r}
\mu^{2,1 \mid 0,0}=\mu^{1,2 \mid 0,0}=(1-\pi) \pi \\
\mu^{2,1 \mid 1,0} \leq \frac{15 \mu^{1,2 \mid 1,0}+10}{14} \\
\mu^{2,1 \mid 0,1} \geq \frac{14 \mu^{1,2 \mid 0,1}-10}{15}
\end{array}\right. \\
\mu^{2,1 \mid 1,1 \geq \mu^{1,2 \mid 1,1}} \\
a^{*}\left(d^{A}, d^{B}\right) \in\left\{\begin{array}{l}
\{0\} \quad\left(d^{A}, d^{B}\right)=(0,0) \\
\{0\} \quad\left(d^{A}, d^{B}\right)=(1,0) \\
\{1\} \quad\left(d^{A}, d^{B}\right)=(0,1) \\
\{1\} \quad\left(d^{A}, d^{B}\right)=(1,1)
\end{array}\right.
\end{gathered}
$$

7.

$$
\left\{d^{A *}\left(p^{A}, p^{B}\right), d^{B *}\left(p^{A}, p^{B}\right)\right\}= \begin{cases}\{0,0\} & \text { if }\left(p^{A}, p^{B}\right)=(1,1) \\ \{0,0\} & \text { if }\left(p^{A}, p^{B}\right)=(2,1) \\ \{0,0\} & \text { if }\left(p^{A}, p^{B}\right)=(1,2) \\ \{0,0\} & \text { if }\left(p^{A}, p^{B}\right)=(2,2)\end{cases}
$$




$$
\begin{aligned}
& \left\{\begin{array}{c}
\mu^{2,1 \mid 0,0}=\mu^{1,2 \mid 0,0}=(1-\pi) \pi \\
\mu^{2,1 \mid 1,0} \geq \frac{15 \mu^{1,2 \mid 1,0}+10}{14} \\
\mu^{2,1 \mid 0,1} \leq \frac{14 \mu^{1,2 \mid 0,1}-10}{15} \\
\mu^{2,1 \mid 1,1} \geq \mu^{1,2 \mid 1,1}
\end{array}\right. \\
& a^{*}\left(d^{A}, d^{B}\right) \in \begin{cases}\{0\} & \left(d^{A}, d^{B}\right)=(0,0) \\
\{1\} & \left(d^{A}, d^{B}\right)=(1,0) \\
\{0\} & \left(d^{A}, d^{B}\right)=(0,1) \\
\{1\} & \left(d^{A}, d^{B}\right)=(1,1)\end{cases}
\end{aligned}
$$

8.

$$
\begin{aligned}
& \left\{d^{A *}\left(p^{A}, p^{B}\right), d^{B *}\left(p^{A}, p^{B}\right)\right\}= \begin{cases}\{0,0\} & \text { if }\left(p^{A}, p^{B}\right)=(1,1) \\
\{0,0\} & \text { if }\left(p^{A}, p^{B}\right)=(2,1) \\
\{0,0\} & \text { if }\left(p^{A}, p^{B}\right)=(1,2) \\
\{0,0\} & \text { if }\left(p^{A}, p^{B}\right)=(2,2)\end{cases} \\
& \left\{\begin{array}{c}
\mu^{2,1 \mid 0,0}=\mu^{1,2 \mid 0,0}=(1-\pi) \pi \\
\mu^{2,1 \mid 1,0} \geq \frac{15 \mu^{1,2 \mid 1,0}+10}{14} \\
\mu^{2,1 \mid 0,1} \geq \frac{14 \mu^{1,2 \mid 0,1}-10}{15} \\
\mu^{2,1 \mid 1,1} \leq \mu^{1,2 \mid 1,1}
\end{array}\right. \\
& a^{*}\left(d^{A}, d^{B}\right) \in \begin{cases}\{0\} & \left(d^{A}, d^{B}\right)=(0,0) \\
\{1\} & \left(d^{A}, d^{B}\right)=(1,0) \\
\{1\} & \left(d^{A}, d^{B}\right)=(0,1) \\
\{0\} & \left(d^{A}, d^{B}\right)=(1,1)\end{cases}
\end{aligned}
$$

9.

$$
\begin{gathered}
\left\{d^{A *}\left(p^{A}, p^{B}\right), d^{B *}\left(p^{A}, p^{B}\right)\right\}=\left\{\begin{array}{l}
\{0,0\} \quad \text { if }\left(p^{A}, p^{B}\right)=(1,1) \\
\{0,0\} \quad \text { if }\left(p^{A}, p^{B}\right)=(2,1) \\
\{0,0\} \quad \text { if }\left(p^{A}, p^{B}\right)=(1,2) \\
\{0,0\} \quad \text { if }\left(p^{A}, p^{B}\right)=(2,2)
\end{array}\right. \\
\left\{\begin{array}{r}
\mu^{2,1 \mid 0,0}=\mu^{1,2 \mid 0,0}=(1-\pi) \pi \\
\mu^{2,1 \mid 1,0} \leq \frac{15 \mu^{1,2 \mid 1,0}+10}{14} \\
\mu^{2,1 \mid 0,1} \leq \frac{14 \mu^{1,2 \mid 0,1}-10}{15} \\
\mu^{2,1 \mid 1,1} \geq \mu^{1,2 \mid 1,1}
\end{array}\right. \\
a^{*}\left(d^{A}, d^{B}\right) \in\left\{\begin{array}{r}
\{1\} \quad\left(d^{A}, d^{B}\right)=(0,0) \\
\{0\} \quad\left(d^{A}, d^{B}\right)=(1,0) \\
\{0\} \quad\left(d^{A}, d^{B}\right)=(0,1) \\
\{1\} \quad\left(d^{A}, d^{B}\right)=(1,1)
\end{array}\right.
\end{gathered}
$$

10.

$$
\left\{d^{A *}\left(p^{A}, p^{B}\right), d^{B *}\left(p^{A}, p^{B}\right)\right\}= \begin{cases}\{0,0\} & \text { if }\left(p^{A}, p^{B}\right)=(1,1) \\ \{0,0\} & \text { if }\left(p^{A}, p^{B}\right)=(2,1) \\ \{0,0\} & \text { if }\left(p^{A}, p^{B}\right)=(1,2) \\ \{0,0\} & \text { if }\left(p^{A}, p^{B}\right)=(2,2)\end{cases}
$$




$$
\begin{aligned}
& \left\{\begin{array}{c}
\mu^{2,1 \mid 0,0}=\mu^{1,2 \mid 0,0}=(1-\pi) \pi \\
\mu^{2,1 \mid 1,0} \leq \frac{15 \mu^{1,2 \mid 1,0}+10}{14} \\
\mu^{2,1 \mid 0,1} \geq \frac{14 \mu^{1,2 \mid 0,1}-10}{15} \\
\mu^{2,1 \mid 1,1} \leq \mu^{1,2 \mid 1,1}
\end{array}\right. \\
& a^{*}\left(d^{A}, d^{B}\right) \in \begin{cases}\{1\} & \left(d^{A}, d^{B}\right)=(0,0) \\
\{0\} & \left(d^{A}, d^{B}\right)=(1,0) \\
\{1\} & \left(d^{A}, d^{B}\right)=(0,1) \\
\{0\} & \left(d^{A}, d^{B}\right)=(1,1)\end{cases}
\end{aligned}
$$

11.

$$
\begin{gathered}
\left\{d^{A *}\left(p^{A}, p^{B}\right), d^{B *}\left(p^{A}, p^{B}\right)\right\}=\left\{\begin{array}{l}
\{0,0\} \quad \text { if }\left(p^{A}, p^{B}\right)=(1,1) \\
\{0,0\} \quad \text { if }\left(p^{A}, p^{B}\right)=(2,1) \\
\{0,0\} \text { if }\left(p^{A}, p^{B}\right)=(1,2) \\
\{0,0\} \quad \text { if }\left(p^{A}, p^{B}\right)=(2,2)
\end{array}\right. \\
\left\{\begin{array}{r}
\mu^{2,1 \mid 0,0}=\mu^{1,2 \mid 0,0}=(1-\pi) \pi \\
\mu^{2,1 \mid 1,0} \geq \frac{15 \mu^{1,2 \mid 1,0}+10}{14} \\
\mu^{2,1 \mid 0,1} \leq \frac{14 \mu^{1,2 \mid 0,1}-10}{15}
\end{array}\right. \\
\mu^{2,1 \mid 1,1} \leq \mu^{1,2 \mid 1,1} \\
a^{*}\left(d^{A}, d^{B}\right) \in\left\{\begin{array}{r}
\{1\} \quad\left(d^{A}, d^{B}\right)=(0,0) \\
\{1\} \quad\left(d^{A}, d^{B}\right)=(1,0) \\
\{0\} \quad\left(d^{A}, d^{B}\right)=(0,1) \\
\{0\} \quad\left(d^{A}, d^{B}\right)=(1,1)
\end{array}\right.
\end{gathered}
$$

12.

$$
\begin{aligned}
& \left\{d^{A *}\left(p^{A}, p^{B}\right), d^{B *}\left(p^{A}, p^{B}\right)\right\}= \begin{cases}\{0,0\} & \text { if }\left(p^{A}, p^{B}\right)=(1,1) \\
\{0,0\} & \text { if }\left(p^{A}, p^{B}\right)=(2,1) \\
\{0,0\} & \text { if }\left(p^{A}, p^{B}\right)=(1,2) \\
\{0,0\} & \text { if }\left(p^{A}, p^{B}\right)=(2,2)\end{cases} \\
& \left\{\begin{array}{c}
\mu^{2,1 \mid 0,0}=\mu^{1,2 \mid 0,0} \\
\mu^{2,1 \mid 1,0} \leq \frac{15 \mu^{1,2 \mid 1,0}+10}{14} \\
\mu^{2,1 \mid 0,1} \leq \frac{14 \mu^{1,2 \mid 0,1}-10}{15} \\
\mu^{2,1 \mid 1,1} \geq \mu^{1,2 \mid 1,1}
\end{array}\right. \\
& a^{*}\left(d^{A}, d^{B}\right) \in \begin{cases}\{0\} & \left(d^{A}, d^{B}\right)=(0,0) \\
\{0\} & \left(d^{A}, d^{B}\right)=(1,0) \\
\{0\} & \left(d^{A}, d^{B}\right)=(0,1) \\
\{1\} & \left(d^{A}, d^{B}\right)=(1,1)\end{cases}
\end{aligned}
$$

13.

$$
\left\{d^{A *}\left(p^{A}, p^{B}\right), d^{B *}\left(p^{A}, p^{B}\right)\right\}= \begin{cases}\{0,0\} & \text { if }\left(p^{A}, p^{B}\right)=(1,1) \\ \{0,0\} & \text { if }\left(p^{A}, p^{B}\right)=(2,1) \\ \{0,0\} & \text { if }\left(p^{A}, p^{B}\right)=(1,2) \\ \{0,0\} & \text { if }\left(p^{A}, p^{B}\right)=(2,2)\end{cases}
$$




$$
\begin{aligned}
& \left\{\begin{array}{c}
\mu^{2,1 \mid 0,0}=\mu^{1,2 \mid 0,0}=(1-\pi) \pi \\
\mu^{2,1 \mid 1,0} \leq \frac{15 \mu^{1,2 \mid 1,0}+10}{14} \\
\mu^{2,1 \mid 0,1} \leq \frac{14 \mu^{1,2 \mid 0,1}-10}{15} \\
\mu^{2,1 \mid 1,1} \leq \mu^{1,2 \mid 1,1}
\end{array}\right. \\
& a^{*}\left(d^{A}, d^{B}\right) \in \begin{cases}\{1\} & \left(d^{A}, d^{B}\right)=(0,0) \\
\{0\} & \left(d^{A}, d^{B}\right)=(1,0) \\
\{0\} & \left(d^{A}, d^{B}\right)=(0,1) \\
\{0\} & \left(d^{A}, d^{B}\right)=(1,1)\end{cases}
\end{aligned}
$$

14.

$$
\begin{gathered}
\left\{d^{A *}\left(p^{A}, p^{B}\right), d^{B *}\left(p^{A}, p^{B}\right)\right\}=\left\{\begin{array}{l}
\{0,0\} \quad \text { if }\left(p^{A}, p^{B}\right)=(1,1) \\
\{0,0\} \quad \text { if }\left(p^{A}, p^{B}=(2,1)\right. \\
\{0,0\} \quad \text { if }\left(p^{A}, p^{B}\right)=(1,2) \\
\{0,0\} \quad \text { if }\left(p^{A}, p^{B}\right)=(2,2)
\end{array}\right. \\
\left\{\begin{array}{r}
\mu^{2,1 \mid 0,0}=\mu^{1,2 \mid 0,0}=(1-\pi) \pi \\
\mu^{2,1 \mid 1,0} \geq \frac{15 \mu^{1,2 \mid 1,0}+10}{14} \\
\mu^{2,1 \mid 0,1} \leq \frac{14 \mu^{1,2 \mid 0,1}-10}{15} \\
\mu^{2,1 \mid 1,1} \leq \mu^{1,2 \mid 1,1}
\end{array}\right. \\
a^{*}\left(d^{A}, d^{B}\right) \in\left\{\begin{array}{l}
\{0\} \quad\left(d^{A}, d^{B}\right)=(0,0) \\
\{1\} \quad\left(d^{A}, d^{B}\right)=(1,0) \\
\{0\} \quad\left(d^{A}, d^{B}\right)=(0,1) \\
\{0\} \quad\left(d^{A}, d^{B}\right)=(1,1)
\end{array}\right.
\end{gathered}
$$

15.

$$
\begin{gathered}
\left\{d^{A *}\left(p^{A}, p^{B}\right), d^{B *}\left(p^{A}, p^{B}\right)\right\}=\left\{\begin{array}{l}
\{0,0\} \quad \text { if }\left(p^{A}, p^{B}\right)=(1,1) \\
\{0,0\} \quad \text { if }\left(p^{A}, p^{B}\right)=(2,1) \\
\{0,0\} \quad \text { if }\left(p^{A}, p^{B}\right)=(1,2) \\
\{0,0\} \quad \text { if }\left(p^{A}, p^{B}\right)=(2,2)
\end{array}\right. \\
\left\{\begin{array}{r}
\mu^{2,1 \mid 0,0}=\mu^{1,2 \mid 0,0}=(1-\pi) \pi \\
\mu^{2,1 \mid 1,0} \leq \frac{15 \mu^{1,2 \mid 1,0}+10}{14} \\
\mu^{2,1 \mid 0,1} \geq \frac{14 \mu^{1,2 \mid 0,1}-10}{15}
\end{array}\right. \\
\mu^{2,1 \mid 1,1} \leq \mu^{1,2 \mid 1,1} \\
a^{*}\left(d^{A}, d^{B}\right) \in\left\{\begin{array}{l}
\{0\} \quad\left(d^{A}, d^{B}\right)=(0,0) \\
\{0\} \quad\left(d^{A}, d^{B}\right)=(1,0) \\
\{1\} \quad\left(d^{A}, d^{B}\right)=(0,1) \\
\{0\} \quad\left(d^{A}, d^{B}\right)=(1,1)
\end{array}\right.
\end{gathered}
$$

16.

$$
\left\{d^{A *}\left(p^{A}, p^{B}\right), d^{B *}\left(p^{A}, p^{B}\right)\right\}= \begin{cases}\{0,0\} & \text { if }\left(p^{A}, p^{B}\right)=(1,1) \\ \{0,0\} & \text { if }\left(p^{A}, p^{B}\right)=(2,1) \\ \{0,0\} & \text { if }\left(p^{A}, p^{B}\right)=(1,2) \\ \{0,0\} & \text { if }\left(p^{A}, p^{B}\right)=(2,2)\end{cases}
$$




$$
\begin{gathered}
\left\{\begin{array}{c}
\mu^{2,1 \mid 0,0} \leq \mu^{1,2 \mid 0,0}=(1-\pi) \pi \\
\mu^{2,1 \mid 1,0} \leq \frac{15 \mu^{1,2 \mid 1,0}+10}{14} \\
\mu^{2,1 \mid 0,1} \leq \frac{14 \mu^{1,2 \mid 0,1}-10}{15} \\
\mu^{2,1 \mid 1,1} \leq \mu^{1,2 \mid 1,1}
\end{array}\right. \\
a^{*}\left(d^{A}, d^{B}\right) \in \begin{cases}\{0\} & \left(d^{A}, d^{B}\right)=(0,0) \\
\{0\} & \left(d^{A}, d^{B}\right)=(1,0) \\
\{0\} & \left(d^{A}, d^{B}\right)=(0,1) \\
\{0\} & \left(d^{A}, d^{B}\right)=(1,1) .\end{cases}
\end{gathered}
$$

Proposition 8 When $\gamma \in\left[\frac{2}{15}, \frac{1}{6}\right]$ there are multiple PBE such that

1.

$$
\begin{gathered}
\left\{d^{A *}\left(p^{A}, p^{B}\right), d^{B *}\left(p^{A}, p^{B}\right)\right\}=\left\{\begin{array}{c}
(1,1) \text { if }\left(p^{A}, p^{B}\right)=(1,1) \\
(0,0) \text { if }\left(p^{A}, p^{B}\right)=(2,1) \\
(0,0) \text { if }\left(p^{A}, p^{B}\right)=(1,2) \\
(0,0) \text { if }\left(p^{A}, p^{B}\right)=(2,2)
\end{array}\right. \\
\left\{\begin{array}{c}
\mu^{2,1 \mid 0,0}=\mu^{1,2 \mid 0,0}=\frac{(1-\pi) \pi}{2(1-\pi) \pi+(1-\pi)^{2}} \\
\mu^{2,1 \mid 1,0} \geq \frac{15 \mu^{1,2 \mid 1,0}+10}{14} \\
\mu^{2,1 \mid 0,1} \leq \frac{14 \mu^{1,2 \mid 0,1}-10}{15} \\
\mu^{2,1 \mid 1,1}=\mu^{1,2 \mid 1,1}=0 \pi
\end{array}\right. \\
a^{*}\left(d^{A}, d^{B}\right) \in\left\{\begin{array}{l}
\{1\} \quad\left(d^{A}, d^{B}\right)=(0,0) \\
\{1\} \quad\left(d^{A}, d^{B}\right)=(1,0) \\
\{0\} \quad\left(d^{A}, d^{B}\right)=(0,1) \\
\{1\} \quad\left(d^{A}, d^{B}\right)=(1,1)
\end{array}\right.
\end{gathered}
$$

2.

$$
\begin{aligned}
& \left\{d^{A *}\left(p^{A}, p^{B}\right), d^{B *}\left(p^{A}, p^{B}\right)\right\} \in \begin{cases}\{(0,1)\} & \text { if }\left(p^{A}, p^{B}\right)=(1,1) \\
\{(0,0)\} & \text { if }\left(p^{A}, p^{B}\right)=(2,1) \\
\{(0,0)\} & \text { if }\left(p^{A}, p^{B}\right)=(1,2) \\
\{(0,0)\} & \text { if }\left(p^{A}, p^{B}\right)=(2,2)\end{cases} \\
& \left\{\begin{array}{c}
\mu^{2,1 \mid 0,0}=\mu^{1,2 \mid 0,0}=\frac{(1-\pi) \pi}{2(1-\pi) \pi+(1-\pi)^{2}} \\
\mu^{2,1 \mid 1,0} \leq \frac{15 \mu^{1,2 \mid 1,0}+10}{14} \\
0=\mu^{2,1 \mid 0,1} \geq \frac{14 \mu^{1,2 \mid 0,1}-10}{\mu^{2,1 \mid 1,1} \geq^{15} \mu^{1,2 \mid 1,1}} \mu^{1,2 \mid 0,1}=0
\end{array}\right. \\
& a^{*}\left(d^{A}, d^{B}\right) \in \begin{cases}\{1\} & \left(d^{A}, d^{B}\right)=(0,0) \\
\{0\} & \left(d^{A}, d^{B}\right)=(1,0) \\
\{1\} & \left(d^{A}, d^{B}\right)=(0,1) \\
\{1\} & \left(d^{A}, d^{B}\right)=(1,1)\end{cases}
\end{aligned}
$$


3.

$$
\begin{aligned}
& \left\{d^{A *}\left(p^{A}, p^{B}\right), d^{B *}\left(p^{A}, p^{B}\right)\right\} \in \begin{cases}\{(1,0)\} & \text { if }\left(p^{A}, p^{B}\right)=(1,1) \\
\{(0,0)\} & \text { if }\left(p^{A}, p^{B}\right)=(2,1) \\
\{(0,0)\} & \text { if }\left(p^{A}, p^{B}\right)=(1,2) \\
\{(0,0)\} & \text { if }\left(p^{A}, p^{B}\right)=(2,2)\end{cases} \\
& \left\{\begin{array}{c}
\mu^{2,1 \mid 0,0}=\mu^{1,2 \mid 0,0}=\frac{(1-\pi) \pi}{2(1-\pi) \pi+(1-\pi)^{2}} \\
0=\mu^{2,1 \mid 1,0} \leq \frac{15 \mu^{1,2 \mid 1,0}+10}{14} \& \mu^{1,2 \mid 1,0}=0 \\
\mu^{2,1 \mid 0,1} \geq \frac{14 \mu^{1,2 \mid 0,1}-10}{15} \\
\mu^{2,1 \mid 1,1} \geq \mu^{1,2 \mid 1,1}
\end{array}\right. \\
& a^{*}\left(d^{A}, d^{B}\right) \in \begin{cases}\{1\} & \left(d^{A}, d^{B}\right)=(0,0) \\
\{0\} & \left(d^{A}, d^{B}\right)=(1,0) \\
\{1\} & \left(d^{A}, d^{B}\right)=(0,1) \\
\{1\} & \left(d^{A}, d^{B}\right)=(1,1)\end{cases}
\end{aligned}
$$

4.

$$
\begin{aligned}
& \left\{d^{A *}\left(p^{A}, p^{B}\right), d^{B *}\left(p^{A}, p^{B}\right)\right\}= \begin{cases}\{1,1\} & \text { if }\left(p^{A}, p^{B}\right)=(1,1) \\
\{0,0\} & \text { if }\left(p^{A}, p^{B}\right)=(2,1) \\
\{0,0\} & \text { if }\left(p^{A}, p^{B}\right)=(1,2) \\
\{0,0\} & \text { if }\left(p^{A}, p^{B}\right)=(2,2)\end{cases} \\
& \left\{\begin{array}{c}
\mu^{2,1 \mid 0,0}=\mu^{1,2 \mid 0,0}=\frac{(1-\pi) \pi}{2(1-\pi) \pi+(1-\pi)^{2}} \\
\mu^{2,1 \mid 1,0} \geq \frac{15 \mu^{1,2 \mid 1,0}+10}{14} \\
\mu^{2,1 \mid 0,1} \leq \frac{14 \mu^{1,2 \mid 0,1}-10}{15} \\
\mu^{2,1 \mid 1,1}=\mu^{1,2 \mid 1,1}==
\end{array}\right. \\
& a^{*}\left(d^{A}, d^{B}\right) \in \begin{cases}\{0\} & \left(d^{A}, d^{B}\right)=(0,0) \\
\{1\} & \left(d^{A}, d^{B}\right)=(1,0) \\
\{0\} & \left(d^{A}, d^{B}\right)=(0,1) \\
\{1\} & \left(d^{A}, d^{B}\right)=(1,1)\end{cases}
\end{aligned}
$$

5.

$$
\begin{aligned}
& \left\{d^{A *}\left(p^{A}, p^{B}\right), d^{B *}\left(p^{A}, p^{B}\right)\right\}= \begin{cases}\{0,1\} & \text { if }\left(p^{A}, p^{B}\right)=(1,1) \\
\{0,0\} & \text { if }\left(p^{A}, p^{B}\right)=(2,1) \\
\{0,0\} & \text { if }\left(p^{A}, p^{B}\right)=(1,2) \\
\{0,0\} & \text { if }\left(p^{A}, p^{B}\right)=(2,2)\end{cases} \\
& \left\{\begin{array}{c}
\mu^{2,1 \mid 0,0}=\mu^{1,2 \mid 0,0}=\frac{(1-\pi) \pi}{2(1-\pi) \pi+(1-\pi)^{2}} \\
\mu^{2,1 \mid 1,0} \leq \frac{15 \mu^{1,2 \mid 1,0}+10}{14} \\
0=\mu^{2,1 \mid 0,1} \geq \frac{14 \mu^{1,2 \mid 0,1}-10}{\mu^{2,1 \mid 1,1}} \leq \mu^{1,2 \mid 0,1}=0 \\
\mu^{15} \mu^{1,2 \mid 1,1}
\end{array}\right.
\end{aligned}
$$




$$
a^{*}\left(d^{A}, d^{B}\right) \in \begin{cases}\{1\} & \left(d^{A}, d^{B}\right)=(0,0) \\ \{0\} & \left(d^{A}, d^{B}\right)=(1,0) \\ \{1\} & \left(d^{A}, d^{B}\right)=(0,1) \\ \{0\} & \left(d^{A}, d^{B}\right)=(1,1)\end{cases}
$$

6.

$$
\begin{aligned}
& \left\{d^{A *}\left(p^{A}, p^{B}\right), d^{B *}\left(p^{A}, p^{B}\right)\right\}= \begin{cases}\{1,0\} & \text { if }\left(p^{A}, p^{B}\right)=(1,1) \\
\{0,0\} & \text { if }\left(p^{A}, p^{B}\right)=(2,1) \\
\{0,0\} & \text { if }\left(p^{A}, p^{B}\right)=(1,2) \\
\{0,0\} & \text { if }\left(p^{A}, p^{B}\right)=(2,2)\end{cases} \\
& \left\{\begin{array}{c}
\mu^{2,1 \mid 0,0}=\mu^{1,2 \mid 0,0}=\frac{(1-\pi) \pi}{2(1-\pi) \pi+(1-\pi)^{2}} \\
0=\mu^{2,1 \mid 1,0} \leq \frac{15 \mu^{1,2 \mid 1,0}+10}{14} \& \mu^{1,2 \mid 1,0}=0 \\
\mu^{2,1 \mid 0,1} \geq \frac{14 \mu^{1,2 \mid 0,1}-10}{15} \\
\mu^{2,1 \mid 1,1} \leq \mu^{1,2 \mid 1,1}
\end{array}\right. \\
& a^{*}\left(d^{A}, d^{B}\right) \in \begin{cases}\{1\} & \left(d^{A}, d^{B}\right)=(0,0) \\
\{0\} & \left(d^{A}, d^{B}\right)=(1,0) \\
\{1\} & \left(d^{A}, d^{B}\right)=(0,1) \\
\{0\} & \left(d^{A}, d^{B}\right)=(1,1)\end{cases}
\end{aligned}
$$

7.

$$
\begin{aligned}
& \left\{d^{A *}\left(p^{A}, p^{B}\right), d^{B *}\left(p^{A}, p^{B}\right)\right\}= \begin{cases}\{1,0\} & \text { if }\left(p^{A}, p^{B}\right)=(1,1) \\
\{1,0\} & \text { if }\left(p^{A}, p^{B}\right)=(2,1) \\
\{0,1\} & \text { if }\left(p^{A}, p^{B}\right)=(1,2) \\
\{0,0\} & \text { if }\left(p^{A}, p^{B}\right)=(2,2)\end{cases} \\
& \left\{\begin{array}{c}
\mu^{2,1 \mid 0,0} \geq \mu^{1,2 \mid 0,0} \\
\mu^{2,1 \mid 1,0}=\frac{(1-\pi) \pi}{2(1-\pi) \pi+(1-\pi)^{2}} \leq \frac{15 \mu^{1,2 \mid 1,0}+10}{14} \& \mu^{1,2 \mid 1,0}=\frac{(1-\pi) \pi}{2(1-\pi) \pi+(1-\pi)^{2}} \\
\mu^{2,1 \mid 0,1} \geq \frac{14 \mu^{1,2 \mid 0,1}-10}{15} \\
\mu^{2,1 \mid 1,1}=0 \leq \mu^{1,2 \mid 1,1}=0
\end{array}\right. \\
& a^{*}\left(d^{A}, d^{B}\right) \in \begin{cases}\{1\} & \left(d^{A}, d^{B}\right)=(0,0) \\
\{0\} & \left(d^{A}, d^{B}\right)=(1,0) \\
\{1\} & \left(d^{A}, d^{B}\right)=(0,1) \\
\{0\} & \left(d^{A}, d^{B}\right)=(1,1)\end{cases}
\end{aligned}
$$

8.

$$
\left\{d^{A *}\left(p^{A}, p^{B}\right), d^{B *}\left(p^{A}, p^{B}\right)\right\}= \begin{cases}\{1,1\} & \text { if }\left(p^{A}, p^{B}\right)=(1,1) \\ \{0,0\} & \text { if }\left(p^{A}, p^{B}\right)=(2,1) \\ \{0,0\} & \text { if }\left(p^{A}, p^{B}\right)=(1,2) \\ \{0,0\} & \text { if }\left(p^{A}, p^{B}\right)=(2,2)\end{cases}
$$




$$
\begin{aligned}
& \left\{\begin{array}{c}
\mu^{2,1 \mid 0,0}=\mu^{1,2 \mid 0,0}=\frac{(1-\pi) \pi}{2(1-\pi) \pi+(1-\pi)^{2}} \\
\mu^{2,1 \mid 1,0} \geq \frac{15 \mu^{1,2 \mid 1,0}+10}{14} \\
\mu^{2,1 \mid 0,1} \leq \frac{14 \mu^{1,2 \mid 0,1}-10}{15} \\
0=\mu^{2,1 \mid 1,1}=\mu^{1,2 \mid 1,1}
\end{array}\right. \\
& a^{*}\left(d^{A}, d^{B}\right) \in \begin{cases}\{0\} & \left(d^{A}, d^{B}\right)=(0,0) \\
\{1\} & \left(d^{A}, d^{B}\right)=(1,0) \\
\{0\} & \left(d^{A}, d^{B}\right)=(0,1) \\
\{0\} & \left(d^{A}, d^{B}\right)=(1,1)\end{cases}
\end{aligned}
$$

9.

$$
\begin{aligned}
& \left\{d^{A *}\left(p^{A}, p^{B}\right), d^{B *}\left(p^{A}, p^{B}\right)\right\}= \begin{cases}\{1,0\} & \text { if }\left(p^{A}, p^{B}\right)=(1,1) \\
\{0,0\} & \text { if }\left(p^{A}, p^{B}\right)=(2,1) \\
\{0,0\} & \text { if }\left(p^{A}, p^{B}\right)=(1,2) \\
\{0,0\} & \text { if }\left(p^{A}, p^{B}\right)=(2,2)\end{cases} \\
& \left\{\begin{array}{c}
\mu^{2,1 \mid 0,0}=\mu^{1,2 \mid 0,0}=\frac{(1-\pi) \pi}{2(1-\pi) \pi+(1-\pi)^{2}} \\
{ }^{2}=\mu^{2,1 \mid 1,0} \leq \frac{15 \mu^{1,2 \mid 1,0}+10}{14} \& \mu^{1,2 \mid 1,0}=0 \\
\mu^{2,1 \mid 0,1} \geq \frac{14 \mu^{1,2 \mid 0,1}-10}{15} \\
\mu^{2,1 \mid 1,1} \leq \mu^{1,2 \mid 1,1}
\end{array}\right. \\
& a^{*}\left(d^{A}, d^{B}\right) \in \begin{cases}\{0\} & \left(d^{A}, d^{B}\right)=(0,0) \\
\{0\} & \left(d^{A}, d^{B}\right)=(1,0) \\
\{1\} & \left(d^{A}, d^{B}\right)=(0,1) \\
\{0\} & \left(d^{A}, d^{B}\right)=(1,1)\end{cases}
\end{aligned}
$$

10.

$$
\begin{aligned}
& \left\{d^{A *}\left(p^{A}, p^{B}\right), d^{B *}\left(p^{A}, p^{B}\right)\right\}= \begin{cases}\{0,1\} & \text { if }\left(p^{A}, p^{B}\right)=(1,1) \\
\{0,0\} & \text { if }\left(p^{A}, p^{B}\right)=(2,1) \\
\{0,0\} & \text { if }\left(p^{A}, p^{B}\right)=(1,2) \\
\{0,0\} & \text { if }\left(p^{A}, p^{B}\right)=(2,2)\end{cases} \\
& \left\{\begin{array}{c}
\mu^{2,1 \mid 0,0}=\mu^{1,2 \mid 0,0}=\frac{(1-\pi) \pi}{2(1-\pi) \pi+(1-\pi)^{2}} \\
\mu^{2,1 \mid 1,0} \leq \frac{15 \mu^{1,2 \mid 1,0}+10}{14} \\
0=\mu^{2,1 \mid 0,1} \geq \frac{14 \mu^{1,2 \mid 0,1}-10}{\mu^{2,1 \mid 1,1}} \leq \mu^{1,2 \mid 0,1}=0 \\
\mu^{1,2 \mid 1,1}
\end{array}\right. \\
& a^{*}\left(d^{A}, d^{B}\right) \in \begin{cases}\{0\} & \left(d^{A}, d^{B}\right)=(0,0) \\
\{0\} & \left(d^{A}, d^{B}\right)=(1,0) \\
\{1\} & \left(d^{A}, d^{B}\right)=(0,1) \\
\{0\} & \left(d^{A}, d^{B}\right)=(1,1)\end{cases}
\end{aligned}
$$


11.

$$
\begin{aligned}
& \left\{d^{A *}\left(p^{A}, p^{B}\right), d^{B *}\left(p^{A}, p^{B}\right)\right\}= \begin{cases}\{0,1\} & \text { if }\left(p^{A}, p^{B}\right)=(1,1) \\
\{0,0\} & \text { if }\left(p^{A}, p^{B}\right)=(2,1) \\
\{0,0\} & \text { if }\left(p^{A}, p^{B}\right)=(1,2) \\
\{0,0\} & \text { if }\left(p^{A}, p^{B}\right)=(2,2)\end{cases} \\
& \left\{\begin{array}{c}
\mu^{2,1 \mid 0,0}=\mu^{1,2 \mid 0,0}=\frac{(1-\pi) \pi}{2(1-\pi) \pi+(1-\pi)^{2}} \\
\mu^{2,1 \mid 1,0} \leq \frac{15 \mu^{1,2 \mid 1,0}+10}{14} \\
\mu^{2,1 \mid 0,1} \geq \frac{14 \mu^{1,2 \mid 0,1}-10}{15} \& \mu^{2,1 \mid 0,1}=\mu^{1,2 \mid 0,1}=0 \\
\mu^{2,1 \mid 1,1} \geq \mu^{1,2 \mid 1,1}
\end{array}\right. \\
& a^{*}\left(d^{A}, d^{B}\right) \in \begin{cases}\{0\} & \left(d^{A}, d^{B}\right)=(0,0) \\
\{0\} & \left(d^{A}, d^{B}\right)=(1,0) \\
\{1\} & \left(d^{A}, d^{B}\right)=(0,1) \\
\{1\} & \left(d^{A}, d^{B}\right)=(1,1)\end{cases}
\end{aligned}
$$

12.

$$
\begin{aligned}
& \left\{d^{A *}\left(p^{A}, p^{B}\right), d^{B *}\left(p^{A}, p^{B}\right)\right\}= \begin{cases}\{1,0\} & \text { if }\left(p^{A}, p^{B}\right)=(1,1) \\
\{0,0\} & \text { if }\left(p^{A}, p^{B}\right)=(2,1) \\
\{0,0\} & \text { if }\left(p^{A}, p^{B}\right)=(1,2) \\
\{0,0\} & \text { if }\left(p^{A}, p^{B}\right)=(2,2)\end{cases} \\
& \left\{\begin{array}{c}
\mu^{2,1 \mid 0,0}=\mu^{1,2 \mid 0,0}=\frac{(1-\pi) \pi}{2(1-\pi) \pi+(1-\pi)^{2}} \\
\mu^{2,1 \mid 1,0} \leq \frac{15 \mu^{1,2 \mid 1,0}+10}{14} \& \mu^{2,1 \mid 1,0}=\mu^{1,2 \mid 1,0}=0 \\
\mu^{2,1 \mid 0,1} \geq \frac{14 \mu^{1,2 \mid 0,1}-10}{\mu^{15}} \\
\mu^{2,1 \mid 1,1} \geq \mu^{1,2 \mid 1,1}
\end{array}\right. \\
& a^{*}\left(d^{A}, d^{B}\right) \in \begin{cases}\{0\} & \left(d^{A}, d^{B}\right)=(0,0) \\
\{0\} & \left(d^{A}, d^{B}\right)=(1,0) \\
\{1\} & \left(d^{A}, d^{B}\right)=(0,1) \\
\{1\} & \left(d^{A}, d^{B}\right)=(1,1)\end{cases}
\end{aligned}
$$

Proposition 9 When $\gamma \in\left[\frac{1}{12}, \frac{2}{15}\right]$ there are multiple PBE such that

1.

$$
\begin{gathered}
\left\{d^{A *}\left(p^{A}, p^{B}\right), d^{B *}\left(p^{A}, p^{B}\right)\right\}=\left\{\begin{array}{l}
\{(1,0)\} \quad \text { if }\left(p^{A}, p^{B}\right)=(1,1) \\
\{(1,0)\} \quad \text { if }\left(p^{A}, p^{B}\right)=(2,1) \\
\{(0,0)\} \quad \text { if }\left(p^{A}, p^{B}\right)=(1,2) \\
\{(0,0)\} \quad \text { if }\left(p^{A}, p^{B}\right)=(2,2)
\end{array}\right. \\
\left\{\begin{array}{c}
0=\mu^{2,1 \mid 0,0} \leq \mu^{1,2 \mid 0,0}=\frac{(1-\pi) \pi}{(1-\pi) \pi+(1-\pi)^{2}} \\
\frac{(1-\pi) \pi}{\pi^{2}+(1-\pi) \pi}=\mu^{2,1 \mid 1,0} \geq \frac{15 \mu^{1,2|1|, 0}+10}{14} \& \mu^{1,2 \mid 1,0}=0 \& \pi \leq \frac{2}{7} \\
\mu^{2,1 \mid 0,1} \geq \frac{14 \mu^{1,2 \mid 0,1}-10}{15} \\
\mu^{2,1 \mid 1,1} \geq \mu^{1,2 \mid 1,1}
\end{array}\right.
\end{gathered}
$$




$$
a^{*}\left(d^{A}, d^{B}\right) \in \begin{cases}\{0\} & \left(d^{A}, d^{B}\right)=(0,0) \\ \{1\} & \left(d^{A}, d^{B}\right)=(1,0) \\ \{1\} & \left(d^{A}, d^{B}\right)=(0,1) \\ \{1\} & \left(d^{A}, d^{B}\right)=(1,1)\end{cases}
$$

2.

$$
\begin{aligned}
& \left\{d^{A *}\left(p^{A}, p^{B}\right), d^{B *}\left(p^{A}, p^{B}\right)\right\}= \begin{cases}\{1,1\} & \text { if }\left(p^{A}, p^{B}\right)=(1,1) \\
\{1,1\} & \text { if }\left(p^{A}, p^{B}\right)=(2,1) \\
\{0,0\} & \text { if }\left(p^{A}, p^{B}\right)=(1,2) \\
\{0,0\} & \text { if }\left(p^{A}, p^{B}\right)=(2,2)\end{cases} \\
& \left\{\begin{array}{c}
\mu^{2,1 \mid 0,0}=0 \leq \mu^{1,2 \mid 0,0}=\frac{(1-\pi) \pi}{(1-\pi) \pi+(1-\pi)^{2}} \\
\mu^{2,1 \mid 1,0} \geq \frac{15 \mu^{1,2 \mid 1,0}+10}{14} \\
\mu^{2,1 \mid 0,1} \leq \frac{14 \mu^{1,2 \mid 0,1}-10}{15} \\
\mu^{2,1 \mid 1,1}=\frac{(1-\pi) \pi}{\pi^{2}+(1-\pi) \pi} \geq \mu^{1,2 \mid 1,1}=0
\end{array}\right. \\
& a^{*}\left(d^{A}, d^{B}\right) \in \begin{cases}\{0\} & \left(d^{A}, d^{B}\right)=(0,0) \\
\{1\} & \left(d^{A}, d^{B}\right)=(1,0) \\
\{0\} & \left(d^{A}, d^{B}\right)=(0,1) \\
\{1\} & \left(d^{A}, d^{B}\right)=(1,1)\end{cases}
\end{aligned}
$$

3.

$$
\begin{aligned}
& \left\{d^{A *}\left(p^{A}, p^{B}\right), d^{B *}\left(p^{A}, p^{B}\right)\right\}= \begin{cases}\{1,0\} & \text { if }\left(p^{A}, p^{B}\right)=(1,1) \\
\{1,0\} & \text { if }\left(p^{A}, p^{B}\right)=(2,1) \\
\{1,1\} & \text { if }\left(p^{A}, p^{B}\right)=(1,2) \\
\{0,0\} & \text { if }\left(p^{A}, p^{B}\right)=(2,2)\end{cases} \\
& \left\{\begin{array}{c}
\mu^{2,1 \mid 0,0}=\mu^{1,2 \mid 0,0}=0 \\
\mu^{2,1 \mid 1,0}=\frac{(1-\pi) \pi}{\pi^{2}+(1-\pi) \pi} \geq \frac{15 \mu^{1,2 \mid 1,0}+10}{14} \& \mu^{1,2 \mid 1,0}=0 \& \pi \leq \frac{2}{7} \\
\mu^{2,1 \mid 0,1} \geq \frac{14 \mu^{1,2 \mid 0,1}-10}{15} \\
0=\mu^{2,1 \mid 1,1} \leq \mu^{1,2 \mid 1,1}=1
\end{array}\right. \\
& a^{*}\left(d^{A}, d^{B}\right) \in \begin{cases}\{0\} & \left(d^{A}, d^{B}\right)=(0,0) \\
\{1\} & \left(d^{A}, d^{B}\right)=(1,0) \\
\{1\} & \left(d^{A}, d^{B}\right)=(0,1) \\
\{0\} & \left(d^{A}, d^{B}\right)=(1,1)\end{cases}
\end{aligned}
$$

4.

$$
\left\{d^{A *}\left(p^{A}, p^{B}\right), d^{B *}\left(p^{A}, p^{B}\right)\right\}= \begin{cases}\{1,1\} & \text { if }\left(p^{A}, p^{B}\right)=(1,1) \\ \{0,0\} & \text { if }\left(p^{A}, p^{B}\right)=(2,1) \\ \{1,1\} & \text { if }\left(p^{A}, p^{B}\right)=(1,2) \\ \{0,0\} & \text { if }\left(p^{A}, p^{B}\right)=(2,2)\end{cases}
$$




$$
\begin{aligned}
& \left\{\begin{array}{c}
\mu^{2,1 \mid 0,0}=\frac{(1-\pi) \pi}{(1-\pi) \pi+(1-\pi)^{2}} \geq \mu^{1,2 \mid 0,0}=0 \\
\mu^{2,1 \mid 1,0} \geq \frac{15 \mu^{1,2 \mid 1,0}+10}{14} \\
\mu^{2,1 \mid 0,1} \leq \frac{14 \mu^{1,2 \mid 0,1}-10}{15} \\
0=\mu^{2,1 \mid 1,1} \leq \mu^{1,2 \mid 1,1}=\frac{(1-\pi) \pi}{\pi^{2}+(1-\pi) \pi}
\end{array}\right. \\
& a^{*}\left(d^{A}, d^{B}\right) \in \begin{cases}\{1\} & \left(d^{A}, d^{B}\right)=(0,0) \\
\{1\} & \left(d^{A}, d^{B}\right)=(1,0) \\
\{0\} & \left(d^{A}, d^{B}\right)=(0,1) \\
\{0\} & \left(d^{A}, d^{B}\right)=(1,1)\end{cases}
\end{aligned}
$$

5.

$$
\begin{gathered}
\left\{d^{A *}\left(p^{A}, p^{B}\right), d^{B *}\left(p^{A}, p^{B}\right)\right\}=\left\{\begin{array}{l}
\{0,1\} \quad \text { if }\left(p^{A}, p^{B}\right)=(1,1) \\
\{1,1\} \quad \text { if }\left(p^{A}, p^{B}\right)=(2,1) \\
\{0,1\} \quad \text { if }\left(p^{A}, p^{B}\right)=(1,2) \\
\{0,0\} \quad \text { if }\left(p^{A}, p^{B}\right)=(2,2)
\end{array}\right. \\
\left\{\begin{array}{c}
0=\mu^{2,1 \mid 0,0}=\mu^{1,2 \mid 0,0}=(1-\pi) \pi \\
\mu^{2,1 \mid 1,0} \leq \frac{15 \mu^{1,2 \mid 1,0}+10}{14}
\end{array}\right. \\
\mu^{2,1 \mid 1,1}=1 \geq \mu^{1,2 \mid 1,1}=0 \\
a^{*}\left(d^{A}, d^{B}\right) \in\left\{\begin{aligned}
\{0\} & \left(d^{A}, d^{B}\right)=(0,0) \\
\{0\} & \left(d^{A}, d^{B}\right)=(1,0) \\
\{0\} & \left(d^{A}, d^{B}\right)=(0,1) \\
\{1\} & \left(d^{A}, d^{B}\right)=(1,1)
\end{aligned}\right.
\end{gathered}
$$

6.

$$
\begin{aligned}
& \left\{d^{A *}\left(p^{A}, p^{B}\right), d^{B *}\left(p^{A}, p^{B}\right)\right\}= \begin{cases}\{0,1\} & \text { if }\left(p^{A}, p^{B}\right)=(1,1) \\
\{0,0\} & \text { if }\left(p^{A}, p^{B}\right)=(2,1) \\
\{0,1\} & \text { if }\left(p^{A}, p^{B}\right)=(1,2) \\
\{0,0\} & \text { if }\left(p^{A}, p^{B}\right)=(2,2)\end{cases} \\
& \left\{\begin{array}{c}
\mu^{2,1 \mid 0,0}=\frac{(1-\pi) \pi}{(1-\pi) \pi+(1-\pi)^{2}} \geq \mu^{1,2 \mid 0,0}=0 \\
\mu^{2,1 \mid 1,0} \leq \frac{15 \mu^{1,2 \mid 1,0}+10}{14} \\
0=\mu^{2,1 \mid 0,1} \leq \frac{14 \mu^{1,2 \mid 0,1}-10}{15} \& \mu^{1,2 \mid 0,1}=\frac{(1-\pi) \pi}{\pi^{2}+(1-\pi) \pi} \& \pi \geq \frac{2}{7} \\
\mu^{2,1 \mid 1,1} \leq \mu^{1,2 \mid 1,1}
\end{array}\right. \\
& a^{*}\left(d^{A}, d^{B}\right) \in \begin{cases}\{1\} & \left(d^{A}, d^{B}\right)=(0,0) \\
\{0\} & \left(d^{A}, d^{B}\right)=(1,0) \\
\{0\} & \left(d^{A}, d^{B}\right)=(0,1) \\
\{0\} & \left(d^{A}, d^{B}\right)=(1,1)\end{cases}
\end{aligned}
$$

Proposition 10 When $\gamma \in\left[0, \frac{1}{12}\right]$ there are multiple PBE such that 
1.

$$
\begin{aligned}
& \left\{d^{A *}\left(p^{A}, p^{B}\right), d^{B *}\left(p^{A}, p^{B}\right)\right\}= \begin{cases}(1,1) & \text { if }\left(p^{A}, p^{B}\right)=(1,1) \\
(1,1) & \text { if }\left(p^{A}, p^{B}\right)=(2,1) \\
(1,1) & \text { if }\left(p^{A}, p^{B}\right)=(1,2) \\
(1,1) & \text { if }\left(p^{A}, p^{B}\right)=(2,2)\end{cases} \\
& \left\{\begin{array}{c}
\mu^{2,1 \mid 0,0} \geq \mu^{1,2 \mid 0,0} \\
\mu^{2,1 \mid 1,0} \geq \frac{15 \mu^{1,2 \mid 1,0}+10}{14} \\
\mu^{2,1 \mid 0,1} \leq \frac{14 \mu^{1,2 \mid 0,1}-10}{15} \\
\mu^{2,1 \mid 1,1}=\mu^{1,2 \mid 1,1}=(1-\pi) \pi
\end{array}\right. \\
& a^{*}\left(d^{A}, d^{B}\right) \in \begin{cases}\{1\} & \left(d^{A}, d^{B}\right)=(0,0) \\
\{1\} & \left(d^{A}, d^{B}\right)=(1,0) \\
\{0\} & \left(d^{A}, d^{B}\right)=(0,1) \\
\{1\} & \left(d^{A}, d^{B}\right)=(1,1)\end{cases}
\end{aligned}
$$

2.

$$
\begin{aligned}
& \left\{d^{A *}\left(p^{A}, p^{B}\right), d^{B *}\left(p^{A}, p^{B}\right)\right\} \in \begin{cases}\{(1,0)\} & \text { if }\left(p^{A}, p^{B}\right)=(1,1) \\
\{(1,0)\} & \text { if }\left(p^{A}, p^{B}\right)=(2,1) \\
\{(0,1)\} & \text { if }\left(p^{A}, p^{B}\right)=(1,2) \\
\{(0,1)\} & \text { if }\left(p^{A}, p^{B}\right)=(2,2)\end{cases} \\
& \left\{\begin{array}{c}
\mu^{2,1 \mid 0,0} \geq \mu^{1,2 \mid 0,0} \\
\frac{(1-\pi) \pi}{\pi^{2}+(1-\pi) \pi}=\mu^{2,1 \mid 1,0} \leq \frac{15 \mu^{1,2 \mid 1,0}+10}{14} \& \mu^{1,2 \mid 1,0}=0 \& \pi \geq \frac{2}{7} \\
0=\mu^{2,1 \mid 0,1} \geq \frac{14 \mu^{1,2 \mid 0,1}-10}{15} \& \mu^{1,2 \mid 0,1}=\frac{(1-\pi) \pi}{(1-\pi) \pi+(1-\pi)^{2}} \& \pi \leq \frac{5}{7} \\
\mu^{2,1 \mid 1,1} \geq \mu^{1,2 \mid 1,1}
\end{array}\right. \\
& a^{*}\left(d^{A}, d^{B}\right) \in \begin{cases}\{1\} & \left(d^{A}, d^{B}\right)=(0,0) \\
\{0\} & \left(d^{A}, d^{B}\right)=(1,0) \\
\{1\} & \left(d^{A}, d^{B}\right)=(0,1) \\
\{1\} & \left(d^{A}, d^{B}\right)=(1,1)\end{cases}
\end{aligned}
$$

3.

$$
\begin{aligned}
& \left\{d^{A *}\left(p^{A}, p^{B}\right), d^{B *}\left(p^{A}, p^{B}\right)\right\} \in \begin{cases}\{(0,1)\} & \text { if }\left(p^{A}, p^{B}\right)=(1,1) \\
\{(1,0)\} & \text { if }\left(p^{A}, p^{B}\right)=(2,1) \\
\{(0,1)\} & \text { if }\left(p^{A}, p^{B}\right)=(1,2) \\
\{(1,0)\} & \text { if }\left(p^{A}, p^{B}\right)=(2,2)\end{cases} \\
& \left\{\begin{array}{c}
\mu^{2,1 \mid 0,0} \geq \mu^{1,2 \mid 0,0} \\
\frac{(1-\pi) \pi}{(1-\pi) \pi+(1-\pi)^{2}}=\mu^{2,1 \mid 1,0} \leq \frac{15 \mu^{1,2 \mid 1,0}+10}{14} \& \mu^{1,2 \mid 1,0}=0 \& \pi \leq \frac{5}{7} \\
0=\mu^{2,1 \mid 0,1} \geq \frac{14 \mu^{1,2 \mid 0,1}-10}{15} \& \mu^{1,2 \mid 0,1}=\frac{(1-\pi) \pi}{\pi^{2}+(1-\pi) \pi} \& \pi \geq \frac{2}{7} \\
\mu^{2,1 \mid 1,1} \geq \mu^{1,2 \mid 1,1}
\end{array}\right.
\end{aligned}
$$




$$
a^{*}\left(d^{A}, d^{B}\right) \in \begin{cases}\{1\} & \left(d^{A}, d^{B}\right)=(0,0) \\ \{0\} & \left(d^{A}, d^{B}\right)=(1,0) \\ \{1\} & \left(d^{A}, d^{B}\right)=(0,1) \\ \{1\} & \left(d^{A}, d^{B}\right)=(1,1)\end{cases}
$$

4.

$$
\begin{aligned}
& \left\{d^{A *}\left(p^{A}, p^{B}\right), d^{B *}\left(p^{A}, p^{B}\right)\right\}= \begin{cases}\{1,0\} & \text { if }\left(p^{A}, p^{B}\right)=(1,1) \\
\{1,0\} & \text { if }\left(p^{A}, p^{B}\right)=(2,1) \\
\{0,1\} & \text { if }\left(p^{A}, p^{B}\right)=(1,2) \\
\{0,1\} & \text { if }\left(p^{A}, p^{B}\right)=(2,2)\end{cases} \\
& \left\{\begin{array}{c}
\mu^{2,1 \mid 0,0} \leq \mu^{1,2 \mid 0,0} \\
\mu^{2,1 \mid 1,0}=\frac{(1-\pi) \pi}{\pi^{2}+(1-\pi) \pi} \leq \frac{15 \mu^{1,2 \mid 1,0}+10}{14} \& \mu^{1,2 \mid 1,0}=0 \& \pi \geq \frac{2}{7} \\
\mu^{2,1 \mid 0,1}=0 \geq \frac{14 \mu^{1,2 \mid 0,1}-10}{15} \& \mu^{1,2 \mid 0,1}=\frac{(1-\pi) \pi}{(1-\pi) \pi+(1-\pi)^{2}} \& \pi \leq \frac{5}{7} \\
\mu^{2,1 \mid 1,1} \geq \mu^{1,2 \mid 1,1}
\end{array}\right. \\
& a^{*}\left(d^{A}, d^{B}\right) \in \begin{cases}\{0\} & \left(d^{A}, d^{B}\right)=(0,0) \\
\{0\} & \left(d^{A}, d^{B}\right)=(1,0) \\
\{1\} & \left(d^{A}, d^{B}\right)=(0,1) \\
\{1\} & \left(d^{A}, d^{B}\right)=(1,1)\end{cases}
\end{aligned}
$$

5.

$$
\begin{aligned}
& \left\{d^{A *}\left(p^{A}, p^{B}\right), d^{B *}\left(p^{A}, p^{B}\right)\right\}= \begin{cases}\{0,1\} & \text { if }\left(p^{A}, p^{B}\right)=(1,1) \\
\{1,0\} & \text { if }\left(p^{A}, p^{B}\right)=(2,1) \\
\{0,1\} & \text { if }\left(p^{A}, p^{B}\right)=(1,2) \\
\{1,0\} & \text { if }\left(p^{A}, p^{B}\right)=(2,2)\end{cases} \\
& \left\{\begin{array}{c}
\mu^{2,1 \mid 0,0} \leq \mu^{1,2 \mid 0,0} \\
\mu^{2,1 \mid 1,0}=\frac{(1-\pi) \pi}{(1-\pi)^{2}+(1-\pi) \pi} \leq \frac{15 \mu^{1,2 \mid 1,0}+10}{14} \& \mu^{1,2 \mid 1,0}=0 \& \pi \leq \frac{5}{7} \\
\mu^{2,1 \mid 0,1}=0 \geq \frac{14 \mu^{1,2 \mid 0,1}-10}{15} \& \mu^{1,2 \mid 0,1}=\frac{(1-\pi) \pi}{(1-\pi) \pi+\pi^{2}} \& \pi \geq \frac{2}{7} \\
\mu^{2,1 \mid 1,1} \geq \mu^{1,2 \mid 1,1}
\end{array}\right. \\
& a^{*}\left(d^{A}, d^{B}\right) \in \begin{cases}\{0\} & \left(d^{A}, d^{B}\right)=(0,0) \\
\{0\} & \left(d^{A}, d^{B}\right)=(1,0) \\
\{1\} & \left(d^{A}, d^{B}\right)=(0,1) \\
\{1\} & \left(d^{A}, d^{B}\right)=(1,1)\end{cases}
\end{aligned}
$$

6.

$$
\left\{d^{A *}\left(p^{A}, p^{B}\right), d^{B *}\left(p^{A}, p^{B}\right)\right\}= \begin{cases}\{1,1\} & \text { if }\left(p^{A}, p^{B}\right)=(1,1) \\ \{1,1\} & \text { if }\left(p^{A}, p^{B}\right)=(2,1) \\ \{1,1\} & \text { if }\left(p^{A}, p^{B}\right)=(1,2) \\ \{1,1\} & \text { if }\left(p^{A}, p^{B}\right)=(2,2)\end{cases}
$$




$$
\begin{gathered}
\left\{\begin{array}{c}
\mu^{2,1 \mid 0,0} \leq \mu^{1,2 \mid 0,0} \\
\mu^{2,1 \mid 1,0} \geq \frac{15 \mu^{1,2 \mid 1,0}+10}{14} \\
\mu^{2,1 \mid 0,1} \leq \frac{14 \mu^{1,2 \mid 0,1}-10}{15} \\
\mu^{2,1 \mid 1,1}=\mu^{1,2 \mid 1,1}=(1-\pi) \pi
\end{array}\right. \\
a^{*}\left(d^{A}, d^{B}\right) \in\left\{\begin{array}{cc}
\{0\} & \left(d^{A}, d^{B}\right)=(0,0) \\
\{1\} & \left(d^{A}, d^{B}\right)=(1,0) \\
\{0\} & \left(d^{A}, d^{B}\right)=(0,1) \\
\{1\} & \left(d^{A}, d^{B}\right)=(1,1)
\end{array}\right.
\end{gathered}
$$

7.

$$
\begin{aligned}
& \left\{d^{A *}\left(p^{A}, p^{B}\right), d^{B *}\left(p^{A}, p^{B}\right)\right\}= \begin{cases}\{0,1\} & \text { if }\left(p^{A}, p^{B}\right)=(1,1) \\
\{1,0\} & \text { if }\left(p^{A}, p^{B}\right)=(2,1) \\
\{0,1\} & \text { if }\left(p^{A}, p^{B}\right)=(1,2) \\
\{1,0\} & \text { if }\left(p^{A}, p^{B}\right)=(2,2)\end{cases} \\
& \left\{\begin{array}{c}
\mu^{2,1 \mid 0,0} \geq \mu^{1,2 \mid 0,0} \\
\frac{(1-\pi) \pi}{(1-\pi) \pi+(1-\pi)^{2}}=\mu^{2,1 \mid 1,0} \leq \frac{15 \mu^{1,2 \mid 1,0}+10}{14} \& \mu^{1,2 \mid 1,0}=0 \& \pi \leq \frac{5}{7} \\
0=\mu^{2,1 \mid 0,1} \geq \frac{14 \mu^{1,2 \mid 0,1}-10}{15} \& \mu^{1,2 \mid 0,1}=\frac{(1-\pi) \pi}{\pi^{2}+(1-\pi) \pi} \& \pi \geq \frac{2}{7} \\
\mu^{2,1 \mid 1,1} \leq \mu^{1,2 \mid 1,1}
\end{array}\right. \\
& a^{*}\left(d^{A}, d^{B}\right) \in \begin{cases}\{1\} & \left(d^{A}, d^{B}\right)=(0,0) \\
\{0\} & \left(d^{A}, d^{B}\right)=(1,0) \\
\{1\} & \left(d^{A}, d^{B}\right)=(0,1) \\
\{0\} & \left(d^{A}, d^{B}\right)=(1,1)\end{cases}
\end{aligned}
$$

8.

$$
\begin{gathered}
\left\{d^{A *}\left(p^{A}, p^{B}\right), d^{B *}\left(p^{A}, p^{B}\right)\right\}=\left\{\begin{array}{l}
\{1,1\} \quad \text { if }\left(p^{A}, p^{B}\right)=(1,1) \\
\{1,1\} \quad \text { if }\left(p^{A}, p^{B}\right)=(2,1) \\
\{1,1\} \quad \text { if }\left(p^{A}, p^{B}\right)=(1,2) \\
\{1,1\} \quad \text { if }\left(p^{A}, p^{B}\right)=(2,2)
\end{array}\right. \\
\left\{\begin{array}{c}
\mu^{2,1 \mid 0,0} \geq \mu^{1,2 \mid 0,0} \\
\mu^{2,1 \mid 1,0} \geq \frac{15 \mu^{1,2 \mid 1,0}+10}{14} \\
\mu^{2,1 \mid 0,1} \leq \frac{14 \mu^{1,2 \mid 0,1}-10}{15} \\
\mu^{2,1 \mid 1,1}=\mu^{1,2 \mid 1,1}=(1-\pi) \pi
\end{array}\right. \\
a^{*}\left(d^{A}, d^{B}\right) \in \begin{cases}\{1\} & \left(d^{A}, d^{B}\right)=(0,0) \\
\{1\} & \left(d^{A}, d^{B}\right)=(1,0) \\
\{0\} & \left(d^{A}, d^{B}\right)=(0,1) \\
\{0\} & \left(d^{A}, d^{B}\right)=(1,1)\end{cases}
\end{gathered}
$$


9.

$$
\begin{gathered}
\left\{d^{A *}\left(p^{A}, p^{B}\right), d^{B *}\left(p^{A}, p^{B}\right)\right\}=\left\{\begin{array}{l}
\{0,1\} \quad \text { if }\left(p^{A}, p^{B}\right)=(1,1) \\
\{1,1\} \quad \text { if }\left(p^{A}, p^{B}\right)=(2,1) \\
\{0,1\} \quad \text { if }\left(p^{A}, p^{B}\right)=(1,2) \\
\{0,1\} \quad \text { if }\left(p^{A}, p^{B}\right)=(2,2)
\end{array}\right. \\
\left\{\begin{array}{c}
\mu^{2,1 \mid 0,0} \leq \mu^{1,2 \mid 0,0} \\
\mu^{2,1 \mid 1,0} \leq \frac{15 \mu^{1,2 \mid 1,0}+10}{14} \\
0=\mu^{2,1 \mid 0,1} \leq \frac{14 \mu^{1,2 \mid 0,1}-10}{15} \& \mu^{1,2 \mid 0,1}=\frac{(1-\pi) \pi}{\pi^{2}+(1-\pi) \pi+(1-\pi)^{2}} \\
\mu^{2,1 \mid 1,1}=1 \geq \mu^{1,2 \mid 1,1}=0
\end{array}\right. \\
a^{*}\left(d^{A}, d^{B}\right) \in \begin{cases}\{0\} & \left(d^{A}, d^{B}\right)=(0,0) \\
\{0\} & \left(d^{A}, d^{B}\right)=(1,0) \\
\{0\} & \left(d^{A}, d^{B}\right)=(0,1) \\
\{1\} & \left(d^{A}, d^{B}\right)=(1,1)\end{cases}
\end{gathered}
$$

10.

$$
\begin{aligned}
& \left\{d^{A *}\left(p^{A}, p^{B}\right), d^{B *}\left(p^{A}, p^{B}\right)\right\}= \begin{cases}\{1,1\} & \text { if }\left(p^{A}, p^{B}\right)=(1,1) \\
\{1,1\} & \text { if }\left(p^{A}, p^{B}\right)=(2,1) \\
\{1,1\} & \text { if }\left(p^{A}, p^{B}\right)=(1,2) \\
\{1,1\} & \text { if }\left(p^{A}, p^{B}\right)=(2,2)\end{cases} \\
& \left\{\begin{array}{c}
\mu^{2,1 \mid 0,0} \leq \mu^{1,2 \mid 0,0} \\
\mu^{2,1 \mid 1,0} \geq \frac{15 \mu^{1,2 \mid 1,0}+10}{14} \\
\mu^{2,1 \mid 0,1} \leq \frac{14 \mu^{1,2 \mid 0,1}-10}{15} \\
\mu^{2,1 \mid 1,1}=\mu^{1,2 \mid 1,1}=(1-\pi) \pi
\end{array}\right. \\
& a^{*}\left(d^{A}, d^{B}\right) \in \begin{cases}\{0\} & \left(d^{A}, d^{B}\right)=(0,0) \\
\{1\} & \left(d^{A}, d^{B}\right)=(1,0) \\
\{0\} & \left(d^{A}, d^{B}\right)=(0,1) \\
\{0\} & \left(d^{A}, d^{B}\right)=(1,1)\end{cases}
\end{aligned}
$$

11.

$$
\begin{gathered}
\left\{d^{A *}\left(p^{A}, p^{B}\right), d^{B *}\left(p^{A}, p^{B}\right)\right\}=\left\{\begin{array}{l}
\{0,1\} \quad \text { if }\left(p^{A}, p^{B}\right)=(1,1) \\
\{1,0\} \quad \text { if }\left(p^{A}, p^{B}\right)=(2,1) \\
\{0,1\} \quad \text { if }\left(p^{A}, p^{B}\right)=(1,2) \\
\{1,0\} \quad \text { if }\left(p^{A}, p^{B}\right)=(2,2)
\end{array}\right. \\
\left\{\begin{array}{c}
\mu^{2,1 \mid 0,0} \leq \mu^{1,2 \mid 0,0} \\
\frac{(1-\pi) \pi}{(1-\pi) \pi+(1-\pi)^{2}}=\mu^{2,1 \mid 1,0} \leq \frac{15 \mu^{1,2 \mid 1,0}+10}{14} \& \mu^{1,2 \mid 1,0}=0 \& \pi \leq \frac{5}{7} \\
0=\mu^{2,1 \mid 0,1} \geq \frac{14 \mu^{1,2 \mid 0,1}-10}{15} \& \mu^{1,2 \mid 0,1}=\frac{(1-\pi) \pi}{\pi^{2}+(1-\pi) \pi} \& \pi \geq \frac{2}{7} \\
\mu^{2,1 \mid 1,1} \leq \mu^{1,2 \mid 1,1}
\end{array}\right.
\end{gathered}
$$




$$
a^{*}\left(d^{A}, d^{B}\right) \in \begin{cases}\{0\} & \left(d^{A}, d^{B}\right)=(0,0) \\ \{0\} & \left(d^{A}, d^{B}\right)=(1,0) \\ \{1\} & \left(d^{A}, d^{B}\right)=(0,1) \\ \{0\} & \left(d^{A}, d^{B}\right)=(1,1)\end{cases}
$$

12.

$$
\begin{aligned}
& \left\{d^{A *}\left(p^{A}, p^{B}\right), d^{B *}\left(p^{A}, p^{B}\right)\right\}= \begin{cases}\{1,0\} & \text { if }\left(p^{A}, p^{B}\right)=(1,1) \\
\{1,0\} & \text { if }\left(p^{A}, p^{B}\right)=(2,1) \\
\{0,1\} & \text { if }\left(p^{A}, p^{B}\right)=(1,2) \\
\{0,1\} & \text { if }\left(p^{A}, p^{B}\right)=(2,2)\end{cases} \\
& \left\{\begin{array}{c}
\mu^{2,1 \mid 0,0} \leq \mu^{1,2 \mid 0,0} \\
\frac{(1-\pi) \pi}{(1-\pi) \pi+\pi^{2}}=\mu^{2,1 \mid 1,0} \leq \frac{15 \mu^{1,2 \mid 1,0}+10}{14} \& \mu^{1,2 \mid 1,0}=0 \& \pi \geq \frac{2}{7} \\
0=\mu^{2,1 \mid 0,1} \geq \frac{14 \mu^{1,2 \mid 0,1}-10}{15} \& \mu^{1,2 \mid 0,1}=\frac{(1-\pi) \pi}{(1-\pi)^{2}+(1-\pi) \pi} \& \pi \leq \frac{5}{7} \\
\mu^{2,1 \mid 1,1} \leq \mu^{1,2 \mid 1,1}
\end{array}\right. \\
& a^{*}\left(d^{A}, d^{B}\right) \in \begin{cases}\{0\} & \left(d^{A}, d^{B}\right)=(0,0) \\
\{0\} & \left(d^{A}, d^{B}\right)=(1,0) \\
\{1\} & \left(d^{A}, d^{B}\right)=(0,1) \\
\{0\} & \left(d^{A}, d^{B}\right)=(1,1)\end{cases}
\end{aligned}
$$

\section{Appendix E: Perfect Bayesian Equilibria of the Game in Scenario 3 with Policy Cooper- ation}

The objective of the Political Union $A \cup B$ is to choose $\left(d^{A}, d^{B}\right)$ such that

$$
\left(d^{A}, d^{B}\right) \in \arg \min \left\{\max \left\{\left(\gamma d^{A}+\frac{a^{*}}{p^{A}+p^{B}+p^{A} d^{A}}\right),\left(\gamma d^{B}+\frac{1-a^{*}}{p^{A}+p^{B}+p^{B} d^{B}}\right)\right\}\right\}
$$

Given the objective function of the Political Union, when there are multiplicity of sequential best replies, we will delete choices that are weakly dominated for the single countries and choose symmetric solutions because of consistency with the idea of a Political Union where choices are reached through consensus building procedures.

Now, consider the 16 possible combination of beliefs and the consequent sequential rational choice of the countries (lemma 1).

1. Suppose

$$
\left\{\begin{array}{c}
\mu^{2,1 \mid 0,0} \geq \mu^{1,2 \mid 0,0} \\
\mu^{2,1 \mid 1,0} \geq \frac{15 \mu^{1,2 \mid 1,0}+10}{14} \\
\mu^{2,1 \mid 0,1} \geq \frac{14 \mu^{1,2 \mid 0,1}-10}{15} \\
\mu^{2,1 \mid 1,1} \geq \mu^{1,2 \mid 1,1}
\end{array}\right.
$$


(a) in $\left(p^{A}, p^{B}\right)=(1,1)$ then

$$
\begin{aligned}
& \max \left\{\left(\gamma d^{A}+\frac{a^{*}}{p^{A}+p^{B}+p^{A} d^{A}}\right),\left(\gamma d^{B}+\frac{1-a^{*}}{p^{A}+p^{B}+p^{B} d^{B}}\right)\right\}= \\
& =\left\{\begin{array} { c l } 
{ \operatorname { m a x } \{ \frac { 1 } { 2 } , 0 \} } & { ( d ^ { A } , d ^ { B } ) = ( 0 , 0 ) } \\
{ \operatorname { m a x } \{ \frac { 1 } { 2 } , \gamma \} } & { ( d ^ { A } , d ^ { B } ) = ( 0 , 1 ) } \\
{ \operatorname { m a x } \{ \gamma + \frac { 1 } { 3 } , 0 \} } & { ( d ^ { A } , d ^ { B } ) = ( 1 , 0 ) } \\
{ \operatorname { m a x } \{ \gamma + \frac { 1 } { 3 } , \gamma \} } & { ( d ^ { A } , d ^ { B } ) = ( 1 , 1 ) }
\end{array} \Rightarrow d ^ { A \cup B * } ( 1 , 1 ) \in \left\{\begin{array}{ll}
\{(1,0)\} & \gamma \in\left[0, \frac{1}{6}\right. \\
\{(0,0)\} & \gamma \in\left[\frac{1}{6}, 1\right]
\end{array}\right.\right.
\end{aligned}
$$

(b) in $\left(p^{A}, p^{B}\right)=(2,1)$ then

$$
\begin{aligned}
& \max \left\{\left(\gamma d^{A}+\frac{a^{*}}{p^{A}+p^{B}+p^{A} d^{A}}\right),\left(\gamma d^{B}+\frac{1-a^{*}}{p^{A}+p^{B}+p^{B} d^{B}}\right)\right\}= \\
& =\left\{\begin{array} { c l } 
{ \operatorname { m a x } \{ \frac { 1 } { 3 } , 0 \} } & { ( d ^ { A } , d ^ { B } ) = ( 0 , 0 ) } \\
{ \operatorname { m a x } \{ \frac { 1 } { 3 } , \gamma \} } & { ( d ^ { A } , d ^ { B } ) = ( 0 , 1 ) } \\
{ \operatorname { m a x } \{ \gamma + \frac { 1 } { 5 } , 0 \} } & { ( d ^ { A } , d ^ { B } ) = ( 1 , 0 ) } \\
{ \operatorname { m a x } \{ \gamma + \frac { 1 } { 5 } , \gamma \} } & { ( d ^ { A } , d ^ { B } ) = ( 1 , 1 ) }
\end{array} \Rightarrow d ^ { A \cup B * } ( 2 , 1 ) \in \left\{\begin{array}{ll}
\{(1,0)\} & \gamma \in\left[0, \frac{2}{15}\right] \\
\{(0,0)\} & \gamma \in\left[\frac{2}{15}, 1\right]
\end{array}\right.\right.
\end{aligned}
$$

(c) in $\left(p^{A}, p^{B}\right)=(1,2)$ then

$$
\begin{aligned}
& \max \left\{\left(\gamma d^{A}+\frac{a^{*}}{p^{A}+p^{B}+p^{A} d^{A}}\right),\left(\gamma d^{B}+\frac{1-a^{*}}{p^{A}+p^{B}+p^{B} d^{B}}\right)\right\}= \\
& =\left\{\begin{array} { c l } 
{ \operatorname { m a x } \{ \frac { 1 } { 3 } , 0 \} } & { ( d ^ { A } , d ^ { B } ) = ( 0 , 0 ) } \\
{ \operatorname { m a x } \{ \frac { 1 } { 3 } , \gamma \} } & { ( d ^ { A } , d ^ { B } ) = ( 0 , 1 ) } \\
{ \operatorname { m a x } \{ \gamma + \frac { 1 } { 4 } , 0 \} } & { ( d ^ { A } , d ^ { B } ) = ( 1 , 0 ) } \\
{ \operatorname { m a x } \{ \gamma + \frac { 1 } { 4 } , \gamma \} } & { ( d ^ { A } , d ^ { B } ) = ( 1 , 1 ) }
\end{array} \Rightarrow d ^ { A \cup B * } ( 1 , 2 ) \in \left\{\begin{array}{ll}
\{(1,0)\} & \gamma \in\left[0, \frac{1}{12}\right] \\
\{(0,0)\} & \gamma \in\left[\frac{1}{12}, 1\right]
\end{array}\right.\right.
\end{aligned}
$$

(d) in $\left(p^{A}, p^{B}\right)=(2,2)$ then

$$
\begin{aligned}
& \max \left\{\left(\gamma d^{A}+\frac{a^{*}}{p^{A}+p^{B}+p^{A} d^{A}}\right),\left(\gamma d^{B}+\frac{1-a^{*}}{p^{A}+p^{B}+p^{B} d^{B}}\right)\right\}= \\
& =\left\{\begin{array}{cl}
\max \left\{\frac{1}{4}, 0\right\} & \left(d^{A}, d^{B}\right)=(0,0) \\
\max \left\{\frac{1}{4}, \gamma\right\} & \left(d^{A}, d^{B}\right)=(0,1) \\
\max \left\{\gamma+\frac{1}{6}, 0\right\} & \left(d^{A}, d^{B}\right)=(1,0) \\
\max \left\{\gamma+\frac{1}{6}, \gamma\right\} & \left(d^{A}, d^{B}\right)=(1,1)
\end{array} \Rightarrow d^{A \cup B *}(2,2) \in\left\{\begin{array}{ll}
\{(1,0)\} & \gamma \in\left[0, \frac{1}{12}\right] \\
\{(0,0)\} & \gamma \in\left[\frac{1}{12}, 1\right]
\end{array}\right]\right.
\end{aligned}
$$

Thus in these beliefs regions, there are the following possible PBE

i. if $\gamma \in\left[0, \frac{1}{12}\right]$

$$
\left\{d^{A *}\left(p^{A}, p^{B}\right), d^{B *}\left(p^{A}, p^{B}\right)\right\} \in \begin{cases}\{(1,0)\} & \text { if }\left(p^{A}, p^{B}\right)=(1,1) \\ \{(1,0)\} & \text { if }\left(p^{A}, p^{B}\right)=(2,1) \\ \{(1,0)\} & \text { if }\left(p^{A}, p^{B}\right)=(1,2) \\ \{(1,0)\} & \text { if }\left(p^{A}, p^{B}\right)=(2,2)\end{cases}
$$


ii. if $\gamma \in\left[\frac{1}{12}, \frac{2}{15}\right]$

$$
\left\{d^{A *}\left(p^{A}, p^{B}\right), d^{B *}\left(p^{A}, p^{B}\right)\right\}= \begin{cases}\{(1,0)\} & \text { if }\left(p^{A}, p^{B}\right)=(1,1) \\ \{(1,0)\} & \text { if }\left(p^{A}, p^{B}\right)=(2,1) \\ \{(0,0)\} & \text { if }\left(p^{A}, p^{B}\right)=(1,2) \\ \{(0,0)\} & \text { if }\left(p^{A}, p^{B}\right)=(2,2)\end{cases}
$$

iii. if $\gamma \in\left[\frac{2}{15}, \frac{1}{6}\right]$

$$
\left\{d^{A *}\left(p^{A}, p^{B}\right), d^{B *}\left(p^{A}, p^{B}\right)\right\}= \begin{cases}\{(1,0)\} & \text { if }\left(p^{A}, p^{B}\right)=(1,1) \\ \{(0,0)\} & \text { if }\left(p^{A}, p^{B}\right)=(2,1) \\ \{(0,0)\} & \text { if }\left(p^{A}, p^{B}\right)=(1,2) \\ \{(0,0)\} & \text { if } \quad\left(p^{A}, p^{B}\right)=(2,2)\end{cases}
$$

iv. if $\gamma \in\left[\frac{1}{6}, 1\right]$

$$
\left\{d^{A *}\left(p^{A}, p^{B}\right), d^{B *}\left(p^{A}, p^{B}\right)\right\}= \begin{cases}\{(0,0)\} & \text { if }\left(p^{A}, p^{B}\right)=(1,1) \\ \{(0,0)\} & \text { if }\left(p^{A}, p^{B}\right)=(2,1) \\ \{(0,0)\} & \text { if }\left(p^{A}, p^{B}\right)=(1,2) \\ \{(0,0)\} & \text { if }\left(p^{A}, p^{B}\right)=(2,2)\end{cases}
$$

Now, we have to go back to terrorists' beliefs. Hence

- when $\gamma \in\left[0, \frac{1}{12}\right]$ then $\mu^{2,1 \mid 1,0}=\mu^{1,2 \mid 1,0}=(1-\pi) \pi$ which means that $\mu^{2,1 \mid 1,0} \geq \frac{15 \mu^{1,2 \mid 1,0}+10}{14}$ is not satisfied, hence we get no PBE.

- when $\gamma \in\left[\frac{1}{12}, \frac{2}{15}\right]$ then $\mu^{2,1 \mid 0,0}=0, \mu^{1,2 \mid 0,0}=\frac{(1-\pi) \pi}{(1-\pi) \pi+(1-\pi)^{2}}$ which means that $\mu^{2,1 \mid 0,0} \geq \mu^{1,2 \mid 0,0}$ is impossible.

- when $\gamma \in\left[\frac{2}{15}, \frac{1}{6}\right]$ then $\mu^{2,1 \mid 1,0}=\mu^{1,2 \mid 1,0}=0$ which means that $\mu^{2,1 \mid 1,0} \geq \frac{15 \mu^{1,2 \mid 1,0}+10}{14}$ is impossible.

- when $\gamma \in\left[\frac{1}{6}, 1\right]$ then $\mu^{2,1 \mid 0,0}=\mu^{1,2 \mid 0,0}=(1-\pi) \pi$ which means that $\mu^{2,1 \mid 0,0} \geq \mu^{1,2 \mid 0,0}$ is satisfied.

2. Suppose

$$
\left\{\begin{array}{c}
\mu^{2,1 \mid 0,0} \geq \mu^{1,2 \mid 0,0} \\
\mu^{2,1 \mid 1,0} \geq \frac{15 \mu^{1,2 \mid 1,0}+10}{14} \\
\mu^{2,1 \mid 0,1} \geq \frac{14 \mu^{1,2 \mid 0,1}-10}{15} \\
\mu^{2,1 \mid 1,1} \leq \mu^{1,2 \mid 1,1}
\end{array}\right.
$$

Following the scheme of point 1, we conclude that in these beliefs regions there are the following possible PBE

(a) i. if $\gamma \in\left[0, \frac{1}{12}\right]$

$$
\left\{d^{A *}\left(p^{A}, p^{B}\right), d^{B *}\left(p^{A}, p^{B}\right)\right\}= \begin{cases}\{1,1\} & \text { if }\left(p^{A}, p^{B}\right)=(1,1) \\ \{1,0\} & \text { if }\left(p^{A}, p^{B}\right)=(2,1) \\ \{1,1\} & \text { if }\left(p^{A}, p^{B}\right)=(1,2) \\ \{1,1\} & \text { if } \quad\left(p^{A}, p^{B}\right)=(2,2)\end{cases}
$$


ii. if $\gamma \in\left[\frac{1}{12}, \frac{2}{15}\right]$

$$
\left\{d^{A *}\left(p^{A}, p^{B}\right), d^{B *}\left(p^{A}, p^{B}\right)\right\}= \begin{cases}\{1,1\} & \text { if }\left(p^{A}, p^{B}\right)=(1,1) \\ \{1,0\} & \text { if }\left(p^{A}, p^{B}\right)=(2,1) \\ \{1,1\} & \text { if }\left(p^{A}, p^{B}\right)=(1,2) \\ \{0,0\} & \text { if }\left(p^{A}, p^{B}\right)=(2,2)\end{cases}
$$

iii. if $\gamma \in\left[\frac{2}{15}, \frac{1}{6}\right]$

$$
\left\{d^{A *}\left(p^{A}, p^{B}\right), d^{B *}\left(p^{A}, p^{B}\right)\right\}= \begin{cases}\{1,1\} & \text { if }\left(p^{A}, p^{B}\right)=(1,1) \\ \{0,0\} & \text { if }\left(p^{A}, p^{B}\right)=(2,1) \\ \{0,0\} & \text { if }\left(p^{A}, p^{B}\right)=(1,2) \\ \{0,0\} & \text { if }\left(p^{A}, p^{B}\right)=(2,2)\end{cases}
$$

iv. if $\gamma \in\left[\frac{1}{6}, 1\right]$

$$
\left\{d^{A *}\left(p^{A}, p^{B}\right), d^{B *}\left(p^{A}, p^{B}\right)\right\}= \begin{cases}\{0,0\} & \text { if }\left(p^{A}, p^{B}\right)=(1,1) \\ \{0,0\} & \text { if }\left(p^{A}, p^{B}\right)=(2,1) \\ \{0,0\} & \text { if }\left(p^{A}, p^{B}\right)=(1,2) \\ \{0,0\} & \text { if }\left(p^{A}, p^{B}\right)=(2,2)\end{cases}
$$

Now, we have to go back to terrorists' beliefs. Hence

- when $\gamma \in\left[0, \frac{1}{12}\right]$ then $\mu^{2,1 \mid 1,0}=1, \mu^{1,2 \mid 1,0}=0$ which means that $\mu^{2,1 \mid 1,0} \geq \frac{15 \mu^{1,2 \mid 1,0}+10}{14}$ is satisfied. Moreover $\mu^{2,1 \mid 1,1}=0, \mu^{1,2 \mid 1,1}=$ $\frac{(1-\pi) \pi}{\pi^{2}+(1-\pi) \pi+(1-\pi)^{2}}$ which means that $\mu^{2,1 \mid 1,1} \leq \mu^{1,2 \mid 1,1}$ is satisfied. Hence we have a PBE

- when $\gamma \in\left[\frac{1}{12}, \frac{2}{15}\right]$ then $\mu^{2,1 \mid 1,0}=1, \mu^{1,2 \mid 1,0}=0$ which means that $\mu^{2,1 \mid 1,0} \geq \frac{15 \mu^{1,2 \mid 1,0}+10}{14}$ is satisfied. Moreover $\mu^{2,1 \mid 0,0}=\mu^{1,2 \mid 0,0}=0$ which means that

$$
\mu^{2,1 \mid 0,0}=\mu^{1,2 \mid 0,0}=0
$$

is satisfied. Finally $\mu^{2,1 \mid 1,1}=0, \mu^{1,2 \mid 1,1}=\frac{(1-\pi) \pi}{\pi^{2}+(1-\pi) \pi}$ which means that $0=\mu^{2,1 \mid 1,1} \leq \mu^{1,2 \mid 1,1}=\frac{(1-\pi) \pi}{\pi^{2}+(1-\pi) \pi}$ is satisfied. Hence we have a PBE

- when $\gamma \in\left[\frac{2}{15}, \frac{1}{6}\right]$ then $\mu^{2,1 \mid 0,0}=\mu^{1,2 \mid 0,0}=\frac{(1-\pi) \pi}{2(1-\pi) \pi+(1-\pi)^{2}} \quad$ which means that $\mu^{2,1 \mid 0,0} \geq \mu^{1,2 \mid 0,0}$ is satisfied. Moreover $\mu^{2,1 \mid 1,1}=\mu^{1,2 \mid 1,1}=$ 0 which means that $\mu^{2,1 \mid 1,1} \leq \mu^{1,2 \mid 1,1}$ is satisfied. Hence we have a $\mathrm{PBE}$

- when $\gamma \in\left[\frac{1}{6}, 1\right]$ then $\mu^{2,1 \mid 0,0}=\mu^{1,2 \mid 0,0}=(1-\pi) \pi$ which means that $\mu^{2,1 \mid 0,0} \geq \mu^{1,2 \mid 0,0}$ is satisfied.

3. Suppose

$$
\left\{\begin{array}{c}
\mu^{2,1 \mid 0,0} \geq \mu^{1,2 \mid 0,0} \\
\mu^{2,1 \mid 1,0} \geq \frac{15 \mu^{1,2 \mid 1,0}+10}{14} \\
\mu^{2,1 \mid 0,1} \leq \frac{14 \mu^{1,2 \mid 0,1}-10}{15} \\
\mu^{2,1 \mid 1,1} \geq \mu^{1,2 \mid 1,1}
\end{array}\right.
$$


Following the scheme of point 1 , we conclude that in these beliefs regions there are the following possible $\mathrm{PBE}$

(a) i. if $\gamma \in\left[0, \frac{1}{12}\right]$

$$
\left\{d^{A *}\left(p^{A}, p^{B}\right), d^{B *}\left(p^{A}, p^{B}\right)\right\}= \begin{cases}(1,1) & \text { if }\left(p^{A}, p^{B}\right)=(1,1) \\ (1,1) & \text { if }\left(p^{A}, p^{B}\right)=(2,1) \\ (1,1) & \text { if }\left(p^{A}, p^{B}\right)=(1,2) \\ (1,1) & \text { if }\left(p^{A}, p^{B}\right)=(2,2)\end{cases}
$$

ii. if $\gamma \in\left[\frac{1}{12}, \frac{2}{15}\right]$

$$
\left\{d^{A *}\left(p^{A}, p^{B}\right), d^{B *}\left(p^{A}, p^{B}\right)\right\}= \begin{cases}(1,1) & \text { if }\left(p^{A}, p^{B}\right)=(1,1) \\ (1,1) & \text { if } \left.\quad p^{A}, p^{B}\right)=(2,1) \\ (0,0) & \text { if }\left(p^{A}, p^{B}\right)=(1,2) \\ (0,0) & \text { if }\left(p^{A}, p^{B}\right)=(2,2)\end{cases}
$$

iii. if $\gamma \in\left[\frac{2}{15}, \frac{1}{6}\right]$

$$
\left\{d^{A *}\left(p^{A}, p^{B}\right), d^{B *}\left(p^{A}, p^{B}\right)\right\}= \begin{cases}(1,1) & \text { if }\left(p^{A}, p^{B}\right)=(1,1) \\ (0,0) & \text { if }\left(p^{A}, p^{B}\right)=(2,1) \\ (0,0) & \text { if }\left(p^{A}, p^{B}\right)=(1,2) \\ (0,0) & \text { if }\left(p^{A}, p^{B}\right)=(2,2)\end{cases}
$$

iv. if $\gamma \in\left[\frac{1}{6}, 1\right]$

$$
\left\{d^{A *}\left(p^{A}, p^{B}\right), d^{B *}\left(p^{A}, p^{B}\right)\right\}= \begin{cases}(0,0) & \text { if }\left(p^{A}, p^{B}\right)=(1,1) \\ (0,0) & \text { if }\left(p^{A}, p^{B}\right)=(2,1) \\ (0,0) & \text { if }\left(p^{A}, p^{B}\right)=(1,2) \\ (0,0) & \text { if }\left(p^{A}, p^{B}\right)=(2,2)\end{cases}
$$

Now, we have to go back to terrorists' beliefs. Hence

- when $\gamma \in\left[0, \frac{1}{12}\right]$ then $\mu^{2,1 \mid 1,1}=\mu^{1,2 \mid 1,1}=(1-\pi) \pi$ which means that $\mu^{2,1 \mid 1,1} \geq \mu^{1,2 \mid 1,1}$ is satisfied, hence we get a PBE.

- when $\gamma \in\left[\frac{1}{12}, \frac{2}{15}\right]$ then $\mu^{2,1 \mid 0,0}=0, \mu^{1,2 \mid 0,0}=\frac{(1-\pi) \pi}{(1-\pi) \pi+(1-\pi)^{2}}$ which means that $\mu^{2,1 \mid 0,0} \geq \mu^{1,2 \mid 0,0}$ is impossible.

- when $\gamma \in\left[\frac{2}{15}, \frac{1}{6}\right]$ then $\mu^{2,1 \mid 1,1}=\mu^{1,2 \mid 1,1}=0$ which means that $\mu^{2,1 \mid 1,1} \geq \mu^{1,2 \mid 1,1}$ is satisfied; moreover $\mu^{2,1 \mid 0,0}=\mu^{1,2 \mid 0,0}=\frac{(1-\pi) \pi}{2(1-\pi) \pi+(1-\pi)^{2}}$ which means that $\mu^{2,1 \mid 0,0} \geq \mu^{1,2 \mid 0,0}$ is satisfied, so that we get a PBE.

- when $\gamma \in\left[\frac{1}{6}, 1\right]$ then $\mu^{2,1 \mid 0,0}=\mu^{1,2 \mid 0,0}=(1-\pi) \pi$ which means that $\mu^{2,1 \mid 0,0} \geq \mu^{1,2 \mid 0,0}$ is satisfied. 
4. Suppose

$$
\left\{\begin{array}{c}
\mu^{2,1 \mid 0,0} \geq \mu^{1,2 \mid 0,0} \\
\mu^{2,1 \mid 1,0} \leq \frac{15 \mu^{1,2 \mid 1,0}+10}{14} \\
\mu^{2,1 \mid 0,1} \geq \frac{14 \mu^{1,2 \mid 0,1}-10}{15} \\
\mu^{2,1 \mid 1,1} \geq \mu^{1,2 \mid 1,1}
\end{array}\right.
$$

Following the scheme of point 1 , we conclude that in these beliefs regions there are the following possible PBE

(a) i. if $\gamma \in\left[0, \frac{1}{12}\right]$

$$
\left\{d^{A *}\left(p^{A}, p^{B}\right), d^{B *}\left(p^{A}, p^{B}\right)\right\} \in\left\{\begin{array}{lll}
\{(1,1)\} & \text { if } & \left(p^{A}, p^{B}\right)=(1,1) \\
\{(1,1)\} & \text { if } & \left(p^{A}, p^{B}\right)=(2,1) \\
\{(0,1)\} & \text { if } & \left(p^{A}, p^{B}\right)=(1,2) \\
\{(1,1)\} & \text { if } & \left(p^{A}, p^{B}\right)=(2,2)
\end{array}\right.
$$

ii. if $\gamma \in\left[\frac{1}{12}, \frac{2}{15}\right]$

$$
\left\{d^{A *}\left(p^{A}, p^{B}\right), d^{B *}\left(p^{A}, p^{B}\right)\right\} \in \begin{cases}\{(1,1)\} & \text { if }\left(p^{A}, p^{B}\right)=(1,1) \\ \{(1,1)\} & \text { if }\left(p^{A}, p^{B}\right)=(2,1) \\ \{(0,1)\} & \text { if }\left(p^{A}, p^{B}\right)=(1,2) \\ \{(0,0)\} & \text { if } \quad\left(p^{A}, p^{B}\right)=(2,2)\end{cases}
$$

iii. if $\gamma \in\left[\frac{2}{15}, \frac{1}{6}\right]$

$$
\left\{d^{A *}\left(p^{A}, p^{B}\right), d^{B *}\left(p^{A}, p^{B}\right)\right\}= \begin{cases}\{(1,1)\} & \text { if }\left(p^{A}, p^{B}\right)=(1,1) \\ \{(0,0)\} & \text { if } \quad\left(p^{A}, p^{B}\right)=(2,1) \\ \{(0,0)\} & \text { if }\left(p^{A}, p^{B}\right)=(1,2) \\ \{(0,0)\} & \text { if } \quad\left(p^{A}, p^{B}\right)=(2,2)\end{cases}
$$

iv. if $\gamma \in\left[\frac{1}{6}, 1\right]$

$$
\left\{d^{A *}\left(p^{A}, p^{B}\right), d^{B *}\left(p^{A}, p^{B}\right)\right\}= \begin{cases}\{(0,0)\} & \text { if }\left(p^{A}, p^{B}\right)=(1,1) \\ \{(0,0)\} & \text { if }\left(p^{A}, p^{B}\right)=(2,1) \\ \{(0,0)\} & \text { if }\left(p^{A}, p^{B}\right)=(1,2) \\ \{(0,0)\} & \text { if }\left(p^{A}, p^{B}\right)=(2,2)\end{cases}
$$

Back to terrorists' beliefs

- when $\gamma \in\left[0, \frac{1}{12}\right]$ then $\mu^{2,1 \mid 0,1}=0, \mu^{1,2 \mid 0,1}=1$ which means that $\mu^{2,1 \mid 0,1} \geq \frac{14 \mu^{1,2 \mid 0,1}-10}{15}$ is impossible.

- when $\gamma \in\left[\frac{1}{12}, \frac{2}{15}\right]$ then $\mu^{2,1 \mid 0,1}=0, \mu^{1,2 \mid 0,1}=1$ which means that $\mu^{2,1 \mid 0,1} \geq \frac{14 \mu^{1,2 \mid 0,1}-10}{15}$ is impossible

- when $\gamma \in\left[\frac{2}{15}, \frac{1}{6}\right]$ then $\mu^{2,1 \mid 0,0}=\mu^{1,2 \mid 0,0}=\frac{(1-\pi) \pi}{2(1-\pi) \pi+(1-\pi)^{2}}$ which means that $\mu^{2,1 \mid 0,0}=\mu^{1,2 \mid 0,0}$ is satisfied. Moreover $\mu^{2,1 \mid 1,1}=\mu^{1,2 \mid 1,1}=$ 0 which means that $\mu^{2,1 \mid 1,1}=\mu^{1,2 \mid 1,1}$ is satisfied. Hence we have an equilibrium 
- when $\gamma \in\left[\frac{1}{6}, 1\right]$ then $\mu^{2,1 \mid 0,0}=\mu^{1,2 \mid 0,0}=(1-\pi) \pi$ which means that $\mu^{2,1 \mid 0,0}=\mu^{1,2 \mid 0,0}$ is satisfied.

5. Suppose

$$
\left\{\begin{array}{c}
\mu^{2,1 \mid 0,0} \leq \mu^{1,2 \mid 0,0} \\
\mu^{2,1 \mid 1,0} \geq \frac{15 \mu^{1,2 \mid 1,0}+10}{14} \\
\mu^{2,1 \mid 0,1} \geq \frac{14 \mu^{1,2 \mid 0,1}-10}{15} \\
\mu^{2,1 \mid 1,1} \geq \mu^{1,2 \mid 1,1}
\end{array}\right.
$$

Following the scheme of point 1 , we conclude that in these beliefs regions there are the following possible PBE

(a) i. if $\gamma \in\left[0, \frac{1}{12}\right]$

$$
\left\{d^{A *}\left(p^{A}, p^{B}\right), d^{B *}\left(p^{A}, p^{B}\right)\right\}= \begin{cases}\{(1,1)\} & \text { if }\left(p^{A}, p^{B}\right)=(1,1) \\ \{(1,1)\} & \text { if }\left(p^{A}, p^{B}\right)=(2,1) \\ \{(1,1)\} & \text { if }\left(p^{A}, p^{B}\right)=(1,2) \\ \{(1,1)\} & \text { if }\left(p^{A}, p^{B}\right)=(2,2)\end{cases}
$$

ii. if $\gamma \in\left[\frac{1}{12}, \frac{2}{15}\right]$

$$
\left\{d^{A *}\left(p^{A}, p^{B}\right), d^{B *}\left(p^{A}, p^{B}\right)\right\}= \begin{cases}\{(1,1)\} & \text { if }\left(p^{A}, p^{B}\right)=(1,1) \\ \{(1,1)\} & \text { if } \quad\left(p^{A}, p^{B}\right)=(2,1) \\ \{(0,0)\} & \text { if }\left(p^{A}, p^{B}\right)=(1,2) \\ \{(0,0)\} & \text { if } \quad\left(p^{A}, p^{B}\right)=(2,2)\end{cases}
$$

iii. if $\gamma \in\left[\frac{2}{15}, \frac{1}{6}\right]$

$$
\left\{d^{A *}\left(p^{A}, p^{B}\right), d^{B *}\left(p^{A}, p^{B}\right)\right\}= \begin{cases}\{(1,1)\} & \text { if }\left(p^{A}, p^{B}\right)=(1,1) \\ \{(0,0)\} & \text { if }\left(p^{A}, p^{B}\right)=(2,1) \\ \{(0,0)\} & \text { if }\left(p^{A}, p^{B}\right)=(1,2) \\ \{(0,0)\} & \text { if } \quad\left(p^{A}, p^{B}\right)=(2,2)\end{cases}
$$

iv. if $\gamma \in\left[\frac{1}{6}, 1\right]$

$$
\left\{d^{A *}\left(p^{A}, p^{B}\right), d^{B *}\left(p^{A}, p^{B}\right)\right\}= \begin{cases}\{(0,0)\} & \text { if }\left(p^{A}, p^{B}\right)=(1,1) \\ \{(0,0)\} & \text { if }\left(p^{A}, p^{B}\right)=(2,1) \\ \{(0,0)\} & \text { if }\left(p^{A}, p^{B}\right)=(1,2) \\ \{(0,0)\} & \text { if } \quad\left(p^{A}, p^{B}\right)=(2,2)\end{cases}
$$

Now, we have to go back to terrorists' beliefs. Hence

- when $\gamma \in\left[0, \frac{1}{12}\right]$ then $\mu^{2,1 \mid 1,1}=\mu^{1,2 \mid 1,1}=(1-\pi) \pi$ which means that $\mu^{2,1 \mid 1,1}=\mu^{1,2 \mid 1,1}$ is satisfied.

- when $\gamma \in\left[\frac{1}{12}, \frac{2}{15}\right]$ then $\mu^{2,1 \mid 1,1}=0, \mu^{1,2 \mid 1,1}=\frac{(1-\pi) \pi}{(1-\pi) \pi+(1-\pi)^{2}}$ which means that $\mu^{2,1 \mid 1,1} \geq \mu^{1,2 \mid 1,1}$ is impossible. 
- when $\gamma \in\left[\frac{2}{15}, \frac{1}{6}\right]$ then $\mu^{2,1 \mid 0,0}=\mu^{1,2 \mid 0,0}=\frac{(1-\pi) \pi}{2(1-\pi) \pi+(1-\pi)^{2}}$ which means that $\mu^{2,1 \mid 0,0}=\mu^{1,2 \mid 0,0}$ is satisfied. Moreover $\mu^{2,1 \mid 1,1}=\mu^{1,2 \mid 1,1}=$ 0 which means that $\mu^{2,1 \mid 1,1}=\mu^{1,2 \mid 1,1}$ is satisfied.

- when $\gamma \in\left[\frac{1}{6}, 1\right]$ then $\mu^{2,1 \mid 0,0}=\mu^{1,2 \mid 0,0}=(1-\pi) \pi$ which means that $\mu^{2,1 \mid 0,0}=\mu^{1,2 \mid 0,0}$ is satisfied.

6. Suppose

$$
\left\{\begin{array}{c}
\mu^{2,1 \mid 0,0} \leq \mu^{1,2 \mid 0,0} \\
\mu^{2,1 \mid 1,0} \leq \frac{15 \mu^{1,2 \mid 1,0}+10}{14} \\
\mu^{2,1 \mid 0,1} \geq \frac{14 \mu^{1,2 \mid 0,1}-10}{15} \\
\mu^{2,1 \mid 1,1} \geq \mu^{1,2 \mid 1,1}
\end{array}\right.
$$

Following the scheme of point 1 , we conclude that in these beliefs regions there are the following possible PBE

(a) i. if $\gamma \in\left[0, \frac{1}{12}\right]$

$$
\left\{d^{A *}\left(p^{A}, p^{B}\right), d^{B *}\left(p^{A}, p^{B}\right)\right\}= \begin{cases}\{(1,1)\} & \text { if }\left(p^{A}, p^{B}\right)=(1,1) \\ \{(1,1)\} & \text { if } \left.\quad p^{A}, p^{B}\right)=(2,1) \\ \{(1,1)\} & \text { if }\left(p^{A}, p^{B}\right)=(1,2) \\ \{(1,1)\} & \text { if } \quad\left(p^{A}, p^{B}\right)=(2,2)\end{cases}
$$

ii. if $\gamma \in\left[\frac{1}{12}, \frac{2}{15}\right]$

$$
\left\{d^{A *}\left(p^{A}, p^{B}\right), d^{B *}\left(p^{A}, p^{B}\right)\right\}= \begin{cases}\{(1,1)\} & \text { if }\left(p^{A}, p^{B}\right)=(1,1) \\ \{(1,1)\} & \text { if } \left.\quad p^{A}, p^{B}\right)=(2,1) \\ \{(1,1)\} & \text { if }\left(p^{A}, p^{B}\right)=(1,2) \\ \{(0,0)\} & \text { if } \quad\left(p^{A}, p^{B}\right)=(2,2)\end{cases}
$$

iii. if $\gamma \in\left[\frac{2}{15}, \frac{1}{6}\right]$

$$
\left\{d^{A *}\left(p^{A}, p^{B}\right), d^{B *}\left(p^{A}, p^{B}\right)\right\}= \begin{cases}\{(1,1)\} & \text { if } \quad\left(p^{A}, p^{B}\right)=(1,1) \\ \{(0,0)\} & \text { if } \quad\left(p^{A}, p^{B}\right)=(2,1) \\ \{(0,0)\} & \text { if }\left(p^{A}, p^{B}\right)=(1,2) \\ \{(0,0)\} & \text { if } \quad\left(p^{A}, p^{B}\right)=(2,2)\end{cases}
$$

iv. if $\gamma \in\left[\frac{1}{6}, 1\right]$

$$
\left\{d^{A *}\left(p^{A}, p^{B}\right), d^{B *}\left(p^{A}, p^{B}\right)\right\}= \begin{cases}\{(0,0)\} & \text { if }\left(p^{A}, p^{B}\right)=(1,1) \\ \{(0,0)\} & \text { if }\left(p^{A}, p^{B}\right)=(2,1) \\ \{(0,0)\} & \text { if }\left(p^{A}, p^{B}\right)=(1,2) \\ \{(0,0)\} & \text { if }\left(p^{A}, p^{B}\right)=(2,2)\end{cases}
$$

Now, we have to go back to terrorists' beliefs. Hence

- when $\gamma \in\left[0, \frac{1}{12}\right]$ then $\mu^{2,1 \mid 1,1}=\mu^{1,2 \mid 1,1}=(1-\pi) \pi$ which means that $\mu^{2,1 \mid 1,1}=\mu^{1,2 \mid 1,1}$ is satisfied. 
- when $\gamma \in\left[\frac{1}{12}, \frac{2}{15}\right]$ then $\mu^{2,1 \mid 1,1}=\mu^{1,2 \mid 1,1}=\frac{(1-\pi) \pi}{\pi^{2}+2(1-\pi) \pi}$ which means that $\mu^{2,1 \mid 1,1}=\mu^{1,2 \mid 1,1}$ is satisfied. Moreover $\mu^{2,1 \mid 0,0}=\mu^{1,2 \mid 0,0}=0$ which means that $\mu^{2,1 \mid 0,0}=\mu^{1,2 \mid 0,0}$ is satisfied.

- when $\gamma \in\left[\frac{2}{15}, \frac{1}{6}\right]$ then $\mu^{2,1 \mid 1,1}=\mu^{1,2 \mid 1,1}=0$ which means that $\mu^{2,1 \mid 1,1}=\mu^{1,2 \mid 1,1}=0$ is satisfied. Moreover $\mu^{2,1 \mid 0,0}=\mu^{1,2 \mid 0,0}=$ $\frac{(1-\pi) \pi}{2(1-\pi) \pi+(1-\pi)^{2}}$ which means that $\mu^{2,1 \mid 0,0}=\mu^{1,2 \mid 0,0}$ is satisfied.

- when $\gamma \in\left[\frac{1}{6}, 1\right]$ then $\mu^{2,1 \mid 0,0}=\mu^{1,2 \mid 0,0}=(1-\pi) \pi$ which means that $\mu^{2,1 \mid 0,0}=\mu^{1,2 \mid 0,0}$ is satisfied.

7. Suppose

$$
\left\{\begin{array}{c}
\mu^{2,1 \mid 0,0} \leq \mu^{1,2 \mid 0,0} \\
\mu^{2,1 \mid 1,0} \geq \frac{15 \mu^{1,2 \mid 1,0}+10}{14} \\
\mu^{2,1 \mid 0,1} \leq \frac{14 \mu^{1,2 \mid 0,1}-10}{15} \\
\mu^{2,1 \mid 1,1} \geq \mu^{1,2 \mid 1,1}
\end{array}\right.
$$

Following the scheme of point 1 , we conclude that in these beliefs regions there are the following possible PBE

(a) i. if $\gamma \in\left[0, \frac{1}{12}\right]$

$$
\left\{d^{A *}\left(p^{A}, p^{B}\right), d^{B *}\left(p^{A}, p^{B}\right)\right\}= \begin{cases}\{1,1\} & \text { if }\left(p^{A}, p^{B}\right)=(1,1) \\ \{1,1\} & \text { if }\left(p^{A}, p^{B}\right)=(2,1) \\ \{1,1\} & \text { if }\left(p^{A}, p^{B}\right)=(1,2) \\ \{1,1\} & \text { if }\left(p^{A}, p^{B}\right)=(2,2)\end{cases}
$$

ii. if $\gamma \in\left[\frac{1}{12}, \frac{2}{15}\right]$

$$
\left\{d^{A *}\left(p^{A}, p^{B}\right), d^{B *}\left(p^{A}, p^{B}\right)\right\}= \begin{cases}\{1,1\} & \text { if }\left(p^{A}, p^{B}\right)=(1,1) \\ \{1,1\} & \text { if }\left(p^{A}, p^{B}\right)=(2,1) \\ \{0,0\} & \text { if }\left(p^{A}, p^{B}\right)=(1,2) \\ \{0,0\} & \text { if }\left(p^{A}, p^{B}\right)=(2,2)\end{cases}
$$

iii. if $\gamma \in\left[\frac{2}{15}, \frac{1}{6}\right]$

$$
\left\{d^{A *}\left(p^{A}, p^{B}\right), d^{B *}\left(p^{A}, p^{B}\right)\right\}= \begin{cases}\{1,1\} & \text { if }\left(p^{A}, p^{B}\right)=(1,1) \\ \{0,0\} & \text { if }\left(p^{A}, p^{B}\right)=(2,1) \\ \{0,0\} & \text { if }\left(p^{A}, p^{B}\right)=(1,2) \\ \{0,0\} & \text { if }\left(p^{A}, p^{B}\right)=(2,2)\end{cases}
$$

iv. if $\gamma \in\left[\frac{1}{6}, 1\right]$

$$
\left\{d^{A *}\left(p^{A}, p^{B}\right), d^{B *}\left(p^{A}, p^{B}\right)\right\}= \begin{cases}\{0,0\} & \text { if }\left(p^{A}, p^{B}\right)=(1,1) \\ \{0,0\} & \text { if }\left(p^{A}, p^{B}\right)=(2,1) \\ \{0,0\} & \text { if }\left(p^{A}, p^{B}\right)=(1,2) \\ \{0,0\} & \text { if }\left(p^{A}, p^{B}\right)=(2,2)\end{cases}
$$


Now, we have to go back to terrorists' beliefs. Hence

- when $\gamma \in\left[0, \frac{1}{12}\right]$ then $\mu^{2,1 \mid 1,1}=\mu^{1,2 \mid 1,1}=(1-\pi) \pi$ which means that $\mu^{2,1 \mid 1,1}=\mu^{1,2 \mid 1,1}$ is satisfied.

- when $\gamma \in\left[\frac{1}{12}, \frac{2}{15}\right]$ then $\mu^{2,1 \mid 1,1}=\frac{(1-\pi) \pi}{\pi^{2}+(1-\pi) \pi}$ and $\mu^{1,2 \mid 1,1}=0$ which means that $\mu^{2,1 \mid 1,1}>\mu^{1,2 \mid 1,1}$ is satisfied. Moreover $\mu^{2,1 \mid 0,0}=0$ and $\mu^{1,2 \mid 0,0}=\frac{(1-\pi) \pi}{(1-\pi) \pi+(1-\pi)^{2}}$ which means that $\mu^{2,1 \mid 0,0}<\mu^{1,2 \mid 0,0}$ is satisfied.

- when $\gamma \in\left[\frac{2}{15}, \frac{1}{6}\right]$ then $\mu^{2,1 \mid 0,0}=\mu^{1,2 \mid 0,0}=\frac{(1-\pi) \pi}{2(1-\pi) \pi+(1-\pi)^{2}}$ which means that $\mu^{2,1 \mid 0,0}=\mu^{1,2 \mid 0,0}$ is satisfied. Moreover $\mu^{2,1 \mid 1,1}=\mu^{1,2 \mid 1,1}=$ 0 which means that $\mu^{2,1 \mid 1,1}=\mu^{1,2 \mid 1,1}$ is satisfied.

- when $\gamma \in\left[\frac{1}{6}, 1\right]$ then $\mu^{2,1 \mid 0,0}=\mu^{1,2 \mid 0,0}=(1-\pi) \pi$ which means that $\mu^{2,1 \mid 0,0}=\mu^{1,2 \mid 0,0}$ is satisfied.

8. Suppose

$$
\left\{\begin{aligned}
\mu^{2,1 \mid 0,0} & \leq \mu^{1,2 \mid 0,0} \\
\mu^{2,1 \mid 1,0} & \geq \frac{15 \mu^{1,2 \mid 1,0}+10}{14} \\
\mu^{2,1 \mid 0,1} \geq \frac{14 \mu^{1,2 \mid 0,1}-10}{15} & \mu^{2,1 \mid 1,1} \leq \mu^{1,2 \mid 1,1}
\end{aligned}\right.
$$

Following the scheme of point 1 , we conclude that in these beliefs regions there are the following possible $\mathrm{PBE}$

(a) i. if $\gamma \in\left[0, \frac{1}{12}\right]$

$$
\left\{d^{A *}\left(p^{A}, p^{B}\right), d^{B *}\left(p^{A}, p^{B}\right)\right\}= \begin{cases}\{1,1\} & \text { if }\left(p^{A}, p^{B}\right)=(1,1) \\ \{1,0\} & \text { if }\left(p^{A}, p^{B}\right)=(2,1) \\ \{1,1\} & \text { if }\left(p^{A}, p^{B}\right)=(1,2) \\ \{1,1\} & \text { if }\left(p^{A}, p^{B}\right)=(2,2)\end{cases}
$$

ii. if $\gamma \in\left[\frac{1}{12}, \frac{2}{15}\right]$

$$
\left\{d^{A *}\left(p^{A}, p^{B}\right), d^{B *}\left(p^{A}, p^{B}\right)\right\}= \begin{cases}\{1,1\} & \text { if }\left(p^{A}, p^{B}\right)=(1,1) \\ \{1,0\} & \text { if }\left(p^{A}, p^{B}\right)=(2,1) \\ \{1,1\} & \text { if }\left(p^{A}, p^{B}\right)=(1,2) \\ \{0,0\} & \text { if }\left(p^{A}, p^{B}\right)=(2,2)\end{cases}
$$

iii. if $\gamma \in\left[\frac{2}{15}, \frac{1}{6}\right]$

$$
\left\{d^{A *}\left(p^{A}, p^{B}\right), d^{B *}\left(p^{A}, p^{B}\right)\right\}= \begin{cases}\{1,1\} & \text { if }\left(p^{A}, p^{B}\right)=(1,1) \\ \{0,0\} & \text { if }\left(p^{A}, p^{B}\right)=(2,1) \\ \{0,0\} & \text { if }\left(p^{A}, p^{B}\right)=(1,2) \\ \{0,0\} & \text { if }\left(p^{A}, p^{B}\right)=(2,2)\end{cases}
$$


iv. if $\gamma \in\left[\frac{1}{6}, 1\right]$

$$
\left\{d^{A *}\left(p^{A}, p^{B}\right), d^{B *}\left(p^{A}, p^{B}\right)\right\}= \begin{cases}\{0,0\} & \text { if }\left(p^{A}, p^{B}\right)=(1,1) \\ \{0,0\} & \text { if }\left(p^{A}, p^{B}\right)=(2,1) \\ \{0,0\} & \text { if }\left(p^{A}, p^{B}\right)=(1,2) \\ \{0,0\} & \text { if }\left(p^{A}, p^{B}\right)=(2,2)\end{cases}
$$

Now, we have to go back to terrorists' beliefs. Hence

- when $\gamma \in\left[0, \frac{1}{12}\right]$ then $\mu^{2,1 \mid 1,0}=1$ and $\mu^{1,2 \mid 1,0}=0$ which means that $\mu^{2,1 \mid 1,0} \geq \frac{15 \mu^{1,2 \mid 1,0}+10}{14}$ is satisfied. Moreover $\mu^{2,1 \mid 1,1}=0$ and $\mu^{1,2 \mid 1,1}=\frac{(1-\pi) \pi}{\pi^{2}+(1-\pi) \pi+(1-\pi)^{2}}$ which means that $\mu^{2,1 \mid 1,1}<\mu^{1,2 \mid 1,1}$ is satisfied.

- when $\gamma \in\left[\frac{1}{12}, \frac{2}{15}\right]$ then $\mu^{2,1 \mid 1,0}=1$ and $\mu^{1,2 \mid 1,0}=0$ which means that $\mu^{2,1 \mid 1,0} \geq \frac{15 \mu^{1,2 \mid 1,0}+10}{14}$ is satisfied. Moreover $\mu^{2,1 \mid 1,1}=0$ and $\mu^{1,2 \mid 1,1}=$ $\frac{(1-\pi) \pi}{\pi^{2}+(1-\pi) \pi}$ and $\mu^{2,1 \mid 1,1}<\mu^{1,2 \mid 1,1}$ is satisfied. Finally $\mu^{2,1 \mid 0,0}=\mu^{1,2 \mid 0,0}=$ 0 which means that $\mu^{2,1 \mid 0,0}=\mu^{1,2 \mid 0,0}$ is satisfied.

- when $\gamma \in\left[\frac{2}{15}, \frac{1}{6}\right]$ then $\mu^{2,1 \mid 1,1}=\mu^{1,2 \mid 1,1}=0$ which means that $\mu^{2,1 \mid 1,1}=\mu^{1,2 \mid 1,1}$ is satisfied. Moreover $\mu^{2,1 \mid 0,0}=\mu^{1,2 \mid 0,0}=\frac{(1-\pi) \pi}{2(1-\pi) \pi+(1-\pi)^{2}}$ which means that $\mu^{2,1 \mid 0,0}=\mu^{1,2 \mid 0,0}$ is satisfied.

- when $\gamma \in\left[\frac{1}{6}, 1\right]$ then $\mu^{2,1 \mid 0,0}=\mu^{1,2 \mid 0,0}=(1-\pi) \pi$ which means that $\mu^{2,1 \mid 0,0}=\mu^{1,2 \mid 0,0}$ is satisfied.

9. Suppose

$$
\left\{\begin{aligned}
\mu^{2,1 \mid 0,0} \geq \mu^{1,2 \mid 0,0} \\
\mu^{2,1 \mid 1,0} \leq \frac{15 \mu^{1,2 \mid 1,0}+10}{14} \\
\mu^{2,1 \mid 0,1} \leq \frac{14 \mu^{1,2 \mid 0,1}-10}{15} \\
\mu^{2,1 \mid 1,1} \geq \mu^{1,2 \mid 1,1}
\end{aligned}\right.
$$

Following the scheme of point 1 , we conclude that in these beliefs regions there are the following possible $\mathrm{PBE}$

(a) i. if $\gamma \in\left[0, \frac{1}{12}\right]$

$$
\left\{d^{A *}\left(p^{A}, p^{B}\right), d^{B *}\left(p^{A}, p^{B}\right)\right\}= \begin{cases}\{1,1\} & \text { if }\left(p^{A}, p^{B}\right)=(1,1) \\ \{1,1\} & \text { if }\left(p^{A}, p^{B}\right)=(2,1) \\ \{0,1\} & \text { if }\left(p^{A}, p^{B}\right)=(1,2) \\ \{1,1\} & \text { if }\left(p^{A}, p^{B}\right)=(2,2)\end{cases}
$$

ii. if $\gamma \in\left[\frac{1}{12}, \frac{2}{15}\right]$

$$
\left\{d^{A *}\left(p^{A}, p^{B}\right), d^{B *}\left(p^{A}, p^{B}\right)\right\}= \begin{cases}\{1,1\} & \text { if }\left(p^{A}, p^{B}\right)=(1,1) \\ \{1,1\} & \text { if }\left(p^{A}, p^{B}\right)=(2,1) \\ \{0,1\} & \text { if }\left(p^{A}, p^{B}\right)=(1,2) \\ \{0,0\} & \text { if }\left(p^{A}, p^{B}\right)=(2,2)\end{cases}
$$


iii. if $\gamma \in\left[\frac{2}{15}, \frac{1}{6}\right]$

$$
\left\{d^{A *}\left(p^{A}, p^{B}\right), d^{B *}\left(p^{A}, p^{B}\right)\right\}= \begin{cases}\{1,1\} & \text { if }\left(p^{A}, p^{B}\right)=(1,1) \\ \{0,0\} & \text { if }\left(p^{A}, p^{B}\right)=(2,1) \\ \{0,0\} & \text { if }\left(p^{A}, p^{B}\right)=(1,2) \\ \{0,0\} & \text { if }\left(p^{A}, p^{B}\right)=(2,2)\end{cases}
$$

iv. if $\gamma \in\left[\frac{1}{6}, 1\right]$

$$
\left\{d^{A *}\left(p^{A}, p^{B}\right), d^{B *}\left(p^{A}, p^{B}\right)\right\}= \begin{cases}\{0,0\} & \text { if }\left(p^{A}, p^{B}\right)=(1,1) \\ \{0,0\} & \text { if }\left(p^{A}, p^{B}\right)=(2,1) \\ \{0,0\} & \text { if }\left(p^{A}, p^{B}\right)=(1,2) \\ \{0,0\} & \text { if }\left(p^{A}, p^{B}\right)=(2,2)\end{cases}
$$

Now, we have to go back to terrorists' beliefs . Hence

- when $\gamma \in\left[0, \frac{1}{12}\right]$ then $\mu^{2,1 \mid 0,1}=0$ and $\mu^{1,2 \mid 0,1}=1$ which means that $\mu^{2,1 \mid 0,1} \leq \frac{14 \mu^{1,2 \mid 0,1}-10}{15}$ is satisfied. Moreover $\mu^{2,1 \mid 1,1}=\frac{(1-\pi) \pi}{\pi^{2}+(1-\pi) \pi+(1-\pi)^{2}}$ and $\mu^{1,2 \mid 1,1}=0$ which means that $\mu^{2,1 \mid 1,1}>\mu^{1,2 \mid 1,1}$ is satisfied.

- when $\gamma \in\left[\frac{1}{12}, \frac{2}{15}\right]$ then $\mu^{2,1 \mid 0,1}=0$ and $\mu^{1,2 \mid 0,1}=1$ which means that $\mu^{2,1 \mid 0,1}<\frac{14 \mu^{1,2 \mid 0,1}-10}{15}$ is satisfied. Moreover $\mu^{2,1 \mid 1,1}=\frac{(1-\pi) \pi}{\pi^{2}+(1-\pi) \pi}$ and $\mu^{1,2 \mid 1,1}=0$ which means that $\mu^{2,1 \mid 1,1}>\mu^{1,2 \mid 1,1}$ is satisfied. Finally $\mu^{2,1 \mid 0,0}=\mu^{1,2 \mid 0,0}=0$ which means that $\mu^{2,1 \mid 0,0}=\mu^{1,2 \mid 0,0}$ is satisfied.

- when $\gamma \in\left[\frac{2}{15}, \frac{1}{6}\right]$ then $\mu^{2,1 \mid 1,1}=\mu^{1,2 \mid 1,1}=0$ which means that $\mu^{2,1 \mid 1,1}=\mu^{1,2 \mid 1,1}$ is satisfied. Finally $\mu^{2,1 \mid 0,0}=\mu^{1,2 \mid 0,0}=\frac{(1-\pi) \pi}{2(1-\pi) \pi+(1-\pi)^{2}}$ which means that $\mu^{2,1 \mid 0,0}=\mu^{1,2 \mid 0,0}$ is satisfied.

- when $\gamma \in\left[\frac{1}{6}, 1\right]$ then $\mu^{2,1 \mid 0,0}=\mu^{1,2 \mid 0,0}=(1-\pi) \pi$ which means that $\mu^{2,1 \mid 0,0}=\mu^{1,2 \mid 0,0}$ is satisfied.

10. Suppose

$$
\left\{\begin{array}{c}
\mu^{2,1 \mid 0,0} \geq \mu^{1,2 \mid 0,0} \\
\mu^{2,1 \mid 1,0} \leq \frac{15 \mu^{1,2 \mid 1,0}+10}{14} \\
\mu^{2,1 \mid 0,1} \geq \frac{14 \mu^{1,2 \mid 0,1}-10}{15} \\
\mu^{2,1 \mid 1,1} \leq \mu^{1,2 \mid 1,1}
\end{array}\right.
$$

Following the scheme of point 1 , we conclude that in these beliefs regions there are the following possible PBE

(a) i. if $\gamma \in\left[0, \frac{1}{12}\right]$

$$
\left\{d^{A *}\left(p^{A}, p^{B}\right), d^{B *}\left(p^{A}, p^{B}\right)\right\}=\left\{\begin{array}{lll}
\{1,1\} & \text { if } \quad\left(p^{A}, p^{B}\right)=(1,1) \\
\{1,0\} & \text { if }\left(p^{A}, p^{B}\right)=(2,1) \\
\{1,1\} & \text { if }\left(p^{A}, p^{B}\right)=(1,2) \\
\{1,1\} & \text { if } \quad\left(p^{A}, p^{B}\right)=(2,2)
\end{array}\right.
$$


ii. if $\gamma \in\left[\frac{1}{12}, \frac{2}{15}\right]$

$$
\left\{d^{A *}\left(p^{A}, p^{B}\right), d^{B *}\left(p^{A}, p^{B}\right)\right\}= \begin{cases}\{1,1\} & \text { if }\left(p^{A}, p^{B}\right)=(1,1) \\ \{1,0\} & \text { if }\left(p^{A}, p^{B}\right)=(2,1) \\ \{1,1\} & \text { if }\left(p^{A}, p^{B}\right)=(1,2) \\ \{0,0\} & \text { if }\left(p^{A}, p^{B}\right)=(2,2)\end{cases}
$$

iii. if $\gamma \in\left[\frac{2}{15}, \frac{1}{6}\right]$

$$
\left\{d^{A *}\left(p^{A}, p^{B}\right), d^{B *}\left(p^{A}, p^{B}\right)\right\}= \begin{cases}\{1,1\} & \text { if }\left(p^{A}, p^{B}\right)=(1,1) \\ \{0,0\} & \text { if }\left(p^{A}, p^{B}\right)=(2,1) \\ \{0,0\} & \text { if }\left(p^{A}, p^{B}\right)=(1,2) \\ \{0,0\} & \text { if }\left(p^{A}, p^{B}\right)=(2,2)\end{cases}
$$

iv. if $\gamma \in\left[\frac{1}{6}, 1\right]$

$$
\left\{d^{A *}\left(p^{A}, p^{B}\right), d^{B *}\left(p^{A}, p^{B}\right)\right\}= \begin{cases}\{0,0\} & \text { if }\left(p^{A}, p^{B}\right)=(1,1) \\ \{0,0\} & \text { if }\left(p^{A}, p^{B}\right)=(2,1) \\ \{0,0\} & \text { if }\left(p^{A}, p^{B}\right)=(1,2) \\ \{0,0\} & \text { if }\left(p^{A}, p^{B}\right)=(2,2)\end{cases}
$$

Now, we have to go back to terrorists' beliefs. Hence

- when $\gamma \in\left[0, \frac{1}{12}\right]$ then $\mu^{2,1 \mid 1,0}=1$ and $\mu^{1,2 \mid 1,0}=0$ which means that $\mu^{2,1 \mid 1,0} \leq \frac{15 \mu^{1,2 \mid 1,0}+10}{14}$ is impossible.

- when $\gamma \in\left[\frac{1}{12}, \frac{2}{15}\right]$ then $\mu^{2,1 \mid 1,0}=1$ and $\mu^{1,2 \mid 1,0}=0$ which means that $\mu^{2,1 \mid 1,0} \leq \frac{15 \mu^{1,2 \mid 1,0}+10}{14}$ is impossible.

- when $\gamma \in\left[\frac{2}{15}, \frac{1}{6}\right]$ then $\mu^{2,1 \mid 1,1}=\mu^{1,2 \mid 1,1}=0$ which means that $\mu^{2,1 \mid 1,1}=\mu^{1,2 \mid 1,1}$ is satisfied. Moreover $\mu^{2,1 \mid 0,0}=\mu^{1,2 \mid 0,0}=\frac{(1-\pi) \pi}{2(1-\pi) \pi+(1-\pi)^{2}}$ which means that $\mu^{2,1 \mid 0,0}=\mu^{1,2 \mid 0,0}$ is satisfied.

- when $\gamma \in\left[\frac{1}{6}, 1\right]$ then $\mu^{2,1 \mid 0,0}=\mu^{1,2 \mid 0,0}=(1-\pi) \pi$ which means that $\mu^{2,1 \mid 0,0}=\mu^{1,2 \mid 0,0}$ is satisfied.

11. Suppose

$$
\left\{\begin{aligned}
& \mu^{2,1 \mid 0,0} \geq \mu^{1,2 \mid 0,0} \\
& \mu^{2,1 \mid 1,0} \geq \frac{15 \mu^{1,2 \mid 1,0}+10}{14} \\
& \mu^{2,1 \mid 0,1} \leq \frac{14 \mu^{1,2 \mid 0,1}-10}{15} \\
& \mu^{2,1 \mid 1,1} \leq \mu^{1,2 \mid 1,1}
\end{aligned}\right.
$$

Following the scheme of point 1 , we conclude that in these beliefs regions there are the following possible PBE

(a) i. if $\gamma \in\left[0, \frac{1}{12}\right]$

$$
\left\{d^{A *}\left(p^{A}, p^{B}\right), d^{B *}\left(p^{A}, p^{B}\right)\right\}= \begin{cases}\{1,1\} & \text { if }\left(p^{A}, p^{B}\right)=(1,1) \\ \{1,1\} & \text { if }\left(p^{A}, p^{B}\right)=(2,1) \\ \{1,1\} & \text { if }\left(p^{A}, p^{B}\right)=(1,2) \\ \{1,1\} & \text { if }\left(p^{A}, p^{B}\right)=(2,2)\end{cases}
$$


ii. if $\gamma \in\left[\frac{1}{12}, \frac{2}{15}\right]$

$$
\left\{d^{A *}\left(p^{A}, p^{B}\right), d^{B *}\left(p^{A}, p^{B}\right)\right\}= \begin{cases}\{1,1\} & \text { if }\left(p^{A}, p^{B}\right)=(1,1) \\ \{0,0\} & \text { if }\left(p^{A}, p^{B}\right)=(2,1) \\ \{1,1\} & \text { if }\left(p^{A}, p^{B}\right)=(1,2) \\ \{0,0\} & \text { if }\left(p^{A}, p^{B}\right)=(2,2)\end{cases}
$$

iii. if $\gamma \in\left[\frac{2}{15}, \frac{1}{6}\right]$

$$
\left\{d^{A *}\left(p^{A}, p^{B}\right), d^{B *}\left(p^{A}, p^{B}\right)\right\}= \begin{cases}\{1,1\} & \text { if }\left(p^{A}, p^{B}\right)=(1,1) \\ \{0,0\} & \text { if }\left(p^{A}, p^{B}\right)=(2,1) \\ \{0,0\} & \text { if }\left(p^{A}, p^{B}\right)=(1,2) \\ \{0,0\} & \text { if }\left(p^{A}, p^{B}\right)=(2,2)\end{cases}
$$

iv. if $\gamma \in\left[\frac{1}{6}, 1\right]$

$$
\left\{d^{A *}\left(p^{A}, p^{B}\right), d^{B *}\left(p^{A}, p^{B}\right)\right\}= \begin{cases}\{0,0\} & \text { if } \quad\left(p^{A}, p^{B}\right)=(1,1) \\ \{0,0\} & \text { if } \quad\left(p^{A}, p^{B}\right)=(2,1) \\ \{0,0\} & \text { if }\left(p^{A}, p^{B}\right)=(1,2) \\ \{0,0\} & \text { if }\left(p^{A}, p^{B}\right)=(2,2)\end{cases}
$$

Now, we have to go back to terrorists' beliefs. Hence

- when $\gamma \in\left[0, \frac{1}{12}\right]$ then $\mu^{2,1 \mid 1,1}=\mu^{1,2 \mid 1,1}=(1-\pi) \pi$ which means that $\mu^{2,1 \mid 1,1}=\mu^{1,2 \mid 1,1}$ is satisfied.

- when $\gamma \in\left[\frac{1}{12}, \frac{2}{15}\right]$ then $\mu^{2,1 \mid 1,1}=0$ and $\mu^{1,2 \mid 1,1}=\frac{(1-\pi) \pi}{\pi^{2}+(1-\pi) \pi}$ which means that $\mu^{2,1 \mid 1,1}<\mu^{1,2 \mid 1,1}$ is satisfied. Moreover $\mu^{2,1 \mid 0,0}=$ $\frac{(1-\pi) \pi}{(1-\pi) \pi+(1-\pi)^{2}}$ and $\mu^{1,2 \mid 0,0}=0$ which means that $\mu^{2,1 \mid 0,0}>\mu^{1,2 \mid 0,0}$ is satisfied

- when $\gamma \in\left[\frac{2}{15}, \frac{1}{6}\right]$ then $\mu^{2,1 \mid 1,1}=\mu^{1,2 \mid 1,1} 0$ which means that $\mu^{2,1 \mid 1,1}=$ $\mu^{1,2 \mid 1,1}$ is satisfied. Moreover $\mu^{2,1 \mid 0,0}=\mu^{1,2 \mid 0,0}=\frac{(1-\pi) \pi}{2(1-\pi) \pi+(1-\pi)^{2}}$ which means that $\mu^{2,1 \mid 0,0}=\mu^{1,2 \mid 0,0}$ is satisfied

- when $\gamma \in\left[\frac{1}{6}, 1\right]$ then $\mu^{2,1 \mid 0,0}=\mu^{1,2 \mid 0,0}=(1-\pi) \pi$ which means that $\mu^{2,1 \mid 0,0} \geq \mu^{1,2 \mid 0,0}$ is satisfied.

12. Suppose

$$
\left\{\begin{array}{c}
\mu^{2,1 \mid 0,0} \leq \mu^{1,2 \mid 0,0} \\
\mu^{2,1 \mid 1,0} \leq \frac{15 \mu^{1,2 \mid 1,0}+10}{14} \\
\mu^{2,1 \mid 0,1} \leq \frac{14 \mu^{1,2 \mid 0,1}-10}{15} \\
\mu^{2,1 \mid 1,1} \geq \mu^{1,2 \mid 1,1}
\end{array}\right.
$$

Following the scheme of point 1 , we conclude that in these beliefs regions there are the following possible PBE 
(a) i. if $\gamma \in\left[0, \frac{1}{12}\right]$

$$
\left\{d^{A *}\left(p^{A}, p^{B}\right), d^{B *}\left(p^{A}, p^{B}\right)\right\}= \begin{cases}\{1,1\} & \text { if }\left(p^{A}, p^{B}\right)=(1,1) \\ \{1,1\} & \text { if }\left(p^{A}, p^{B}\right)=(2,1) \\ \{0,1\} & \text { if }\left(p^{A}, p^{B}\right)=(1,2) \\ \{1,1\} & \text { if }\left(p^{A}, p^{B}\right)=(2,2)\end{cases}
$$

ii. if $\gamma \in\left[\frac{1}{12}, \frac{2}{15}\right]$

$$
\left\{d^{A *}\left(p^{A}, p^{B}\right), d^{B *}\left(p^{A}, p^{B}\right)\right\}= \begin{cases}\{1,1\} & \text { if }\left(p^{A}, p^{B}\right)=(1,1) \\ \{1,1\} & \text { if }\left(p^{A}, p^{B}\right)=(2,1) \\ \{0,1\} & \text { if }\left(p^{A}, p^{B}\right)=(1,2) \\ \{0,0\} & \text { if }\left(p^{A}, p^{B}\right)=(2,2)\end{cases}
$$

iii. if $\gamma \in\left[\frac{2}{15}, \frac{1}{6}\right]$

$$
\left\{d^{A *}\left(p^{A}, p^{B}\right), d^{B *}\left(p^{A}, p^{B}\right)\right\}= \begin{cases}\{1,1\} & \text { if }\left(p^{A}, p^{B}\right)=(1,1) \\ \{0,0\} & \text { if }\left(p^{A}, p^{B}\right)=(2,1) \\ \{0,0\} & \text { if }\left(p^{A}, p^{B}\right)=(1,2) \\ \{0,0\} & \text { if }\left(p^{A}, p^{B}\right)=(2,2)\end{cases}
$$

iv. if $\gamma \in\left[\frac{1}{6}, 1\right]$

$$
\left\{d^{A *}\left(p^{A}, p^{B}\right), d^{B *}\left(p^{A}, p^{B}\right)\right\}= \begin{cases}\{0,0\} & \text { if }\left(p^{A}, p^{B}\right)=(1,1) \\ \{0,0\} & \text { if }\left(p^{A}, p^{B}\right)=(2,1) \\ \{0,0\} & \text { if }\left(p^{A}, p^{B}\right)=(1,2) \\ \{0,0\} & \text { if }\left(p^{A}, p^{B}\right)=(2,2)\end{cases}
$$

Now, we have to go back to terrorists' beliefs. Hence

- when $\gamma \in\left[0, \frac{1}{12}\right]$ then $\mu^{2,1 \mid 1,1}=\frac{(1-\pi) \pi}{\pi^{2}+(1-\pi) \pi+(1-\pi)^{2}}$ and $\mu^{1,2 \mid 1,1}=0$ which means that $\mu^{2,1 \mid 1,1}>\mu^{1,2 \mid 1,1}$ is satisfied. Moreover $\mu^{2,1 \mid 0,1}=0$ and $\mu^{1,2 \mid 0,1}=1$ which means that $\mu^{2,1 \mid 0,1} \leq \frac{14 \mu^{1,2 \mid 0,1}-10}{15}$ is satisfied.

- when $\gamma \in\left[\frac{1}{12}, \frac{2}{15}\right]$ then $\mu^{2,1 \mid 0,1}=0$ and $\mu^{1,2 \mid 0,1}=1$ which means that $\mu^{2,1 \mid 0,1} \leq \frac{14 \mu^{1,2 \mid 0,1}-10}{14}$ is satisfied. Moreover $\mu^{2,1 \mid 0,0}=\mu^{1,2 \mid 0,0}=0$ which means that $\mu^{2,1 \mid 0,0}=\mu^{1,2 \mid 0,0}$ is satisfied. Finally $\mu^{2,1 \mid 1,1}=$ $\frac{(1-\pi) \pi}{\pi^{2}+(1-\pi) \pi}$ and $\mu^{1,2 \mid 1,1}=0$ which means that $\mu^{2,1 \mid 1,1}>\mu^{1,2 \mid 1,1}$ is satisfied.

- when $\gamma \in\left[\frac{2}{15}, \frac{1}{6}\right]$ then $\mu^{2,1 \mid 0,0}=\mu^{1,2 \mid 0,0}=\frac{(1-\pi) \pi}{2(1-\pi) \pi+(1-\pi)^{2}}$ which means that $\mu^{2,1 \mid 0,0}=\mu^{1,2 \mid 0,0}$ is satisfied. Finally $\mu^{2,1 \mid 1,1}=\mu^{1,2 \mid 1,1}=$ 0 which means that $\mu^{2,1 \mid 1,1}=\mu^{1,2 \mid 1,1}$ is satisfied.

- when $\gamma \in\left[\frac{1}{6}, 1\right]$ then $\mu^{2,1 \mid 0,0}=\mu^{1,2 \mid 0,0}=(1-\pi) \pi$ which means that $\mu^{2,1 \mid 0,0}=\mu^{1,2 \mid 0,0}$ is satisfied. 
13. Suppose

$$
\left\{\begin{aligned}
\mu^{2,1 \mid 0,0} & \geq \mu^{1,2 \mid 0,0} \\
\mu^{2,1 \mid 1,0} & \leq \frac{15 \mu^{1,2 \mid 1,0}+10}{14} \\
\mu^{2,1 \mid 0,1} & \leq \frac{14 \mu^{1,2 \mid 0,1}-10}{15} \\
\mu^{2,1 \mid 1,1} & \leq \mu^{1,2 \mid 1,1}
\end{aligned}\right.
$$

Following the scheme of point 1 , we conclude that in these beliefs regions there are the following possible PBE

(a) i. if $\gamma \in\left[0, \frac{1}{12}\right]$

$$
\left\{d^{A *}\left(p^{A}, p^{B}\right), d^{B *}\left(p^{A}, p^{B}\right)\right\}= \begin{cases}\{1,1\} & \text { if }\left(p^{A}, p^{B}\right)=(1,1) \\ \{1,1\} & \text { if }\left(p^{A}, p^{B}\right)=(2,1) \\ \{1,1\} & \text { if }\left(p^{A}, p^{B}\right)=(1,2) \\ \{1,1\} & \text { if }\left(p^{A}, p^{B}\right)=(2,2)\end{cases}
$$

ii. if $\gamma \in\left[\frac{1}{12}, \frac{2}{15}\right]$

$$
\left\{d^{A *}\left(p^{A}, p^{B}\right), d^{B *}\left(p^{A}, p^{B}\right)\right\}= \begin{cases}\{1,1\} & \text { if }\left(p^{A}, p^{B}\right)=(1,1) \\ \{0,0\} & \text { if }\left(p^{A}, p^{B}\right)=(2,1) \\ \{1,1\} & \text { if }\left(p^{A}, p^{B}\right)=(1,2) \\ \{0,0\} & \text { if }\left(p^{A}, p^{B}\right)=(2,2)\end{cases}
$$

iii. if $\gamma \in\left[\frac{2}{15}, \frac{1}{6}\right]$

$$
\left\{d^{A *}\left(p^{A}, p^{B}\right), d^{B *}\left(p^{A}, p^{B}\right)\right\}= \begin{cases}\{1,1\} & \text { if }\left(p^{A}, p^{B}\right)=(1,1) \\ \{0,0\} & \text { if }\left(p^{A}, p^{B}\right)=(2,1) \\ \{0,0\} & \text { if }\left(p^{A}, p^{B}\right)=(1,2) \\ \{0,0\} & \text { if }\left(p^{A}, p^{B}\right)=(2,2)\end{cases}
$$

iv. if $\gamma \in\left[\frac{1}{6}, 1\right]$

$$
\left\{d^{A *}\left(p^{A}, p^{B}\right), d^{B *}\left(p^{A}, p^{B}\right)\right\}= \begin{cases}\{0,0\} & \text { if }\left(p^{A}, p^{B}\right)=(1,1) \\ \{0,0\} & \text { if }\left(p^{A}, p^{B}\right)=(2,1) \\ \{0,0\} & \text { if }\left(p^{A}, p^{B}\right)=(1,2) \\ \{0,0\} & \text { if }\left(p^{A}, p^{B}\right)=(2,2)\end{cases}
$$

Now, we have to go back to terrorists' beliefs. Hence

- when $\gamma \in\left[0, \frac{1}{12}\right]$ then $\mu^{2,1 \mid 1,1}=\mu^{1,2 \mid 1,1}=(1-\pi) \pi$ which means that $\mu^{2,1 \mid 1,1}=\mu^{1,2 \mid 1,1}$ is satisfied.

- when $\gamma \in\left[\frac{1}{12}, \frac{2}{15}\right]$ then $\mu^{2,1 \mid 0,0}=\frac{(1-\pi) \pi}{(1-\pi) \pi+(1-\pi)^{2}}$ and $\mu^{1,2 \mid 0,0}=0$ which means that $\mu^{2,1 \mid 0,0}>\mu^{1,2 \mid 0,0}$ is satisfied. Moreover $\mu^{2,1 \mid 1,1}=$ 0 and $\mu^{1,2 \mid 1,1}=\frac{(1-\pi) \pi}{\pi^{2}+(1-\pi) \pi}$ which means that $\mu^{2,1 \mid 1,1}<\mu^{1,2 \mid 1,1}$ is satisfied. 
- when $\gamma \in\left[\frac{2}{15}, \frac{1}{6}\right]$ then $\mu^{2,1 \mid 0,0}=\mu^{1,2 \mid 0,0}=\frac{(1-\pi) \pi}{2(1-\pi) \pi+(1-\pi)^{2}}$ which means that $\mu^{2,1 \mid 0,0}=\mu^{1,2 \mid 0,0}$ is satisfied. Moreover $\mu^{2,1 \mid 1,1}=\mu^{1,2 \mid 1,1}=$ 0 which means that $\mu^{2,1 \mid 1,1}=\mu^{1,2 \mid 1,1}$ is satisfied.

- when $\gamma \in\left[\frac{1}{6}, 1\right]$ then $\mu^{2,1 \mid 0,0}=\mu^{1,2 \mid 0,0}=(1-\pi) \pi$ which means that $\mu^{2,1 \mid 0,0}=\mu^{1,2 \mid 0,0}$ is satisfied.

14. Suppose

$$
\left\{\begin{array}{c}
\mu^{2,1 \mid 0,0} \leq \mu^{1,2 \mid 0,0} \\
\mu^{2,1 \mid 1,0} \geq \frac{15 \mu^{1,2 \mid 1,0}+10}{14} \\
\mu^{2,1 \mid 0,1} \leq \frac{14 \mu^{1,2 \mid 0,1}-10}{15} \\
\mu^{2,1 \mid 1,1} \leq \mu^{1,2 \mid 1,1}
\end{array}\right.
$$

Following the scheme of point 1 , we conclude that in these beliefs regions there are the following possible PBE

(a) i. if $\gamma \in\left[0, \frac{1}{12}\right]$

$$
\left\{d^{A *}\left(p^{A}, p^{B}\right), d^{B *}\left(p^{A}, p^{B}\right)\right\}= \begin{cases}\{1,1\} & \text { if }\left(p^{A}, p^{B}\right)=(1,1) \\ \{1,1\} & \text { if }\left(p^{A}, p^{B}\right)=(2,1) \\ \{1,1\} & \text { if }\left(p^{A}, p^{B}\right)=(1,2) \\ \{1,1\} & \text { if }\left(p^{A}, p^{B}\right)=(2,2)\end{cases}
$$

ii. if $\gamma \in\left[\frac{1}{12}, \frac{2}{15}\right]$

$$
\left\{d^{A *}\left(p^{A}, p^{B}\right), d^{B *}\left(p^{A}, p^{B}\right)\right\}= \begin{cases}\{1,1\} & \text { if }\left(p^{A}, p^{B}\right)=(1,1) \\ \{0,0\} & \text { if }\left(p^{A}, p^{B}\right)=(2,1) \\ \{1,1\} & \text { if }\left(p^{A}, p^{B}\right)=(1,2) \\ \{0,0\} & \text { if }\left(p^{A}, p^{B}\right)=(2,2)\end{cases}
$$

iii. if $\gamma \in\left[\frac{2}{15}, \frac{1}{6}\right]$

$$
\left\{d^{A *}\left(p^{A}, p^{B}\right), d^{B *}\left(p^{A}, p^{B}\right)\right\}= \begin{cases}\{1,1\} & \text { if }\left(p^{A}, p^{B}\right)=(1,1) \\ \{0,0\} & \text { if }\left(p^{A}, p^{B}\right)=(2,1) \\ \{0,0\} & \text { if }\left(p^{A}, p^{B}\right)=(1,2) \\ \{0,0\} & \text { if }\left(p^{A}, p^{B}\right)=(2,2)\end{cases}
$$

iv. if $\gamma \in\left[\frac{1}{6}, 1\right]$

$$
\left\{d^{A *}\left(p^{A}, p^{B}\right), d^{B *}\left(p^{A}, p^{B}\right)\right\}= \begin{cases}\{0,0\} & \text { if }\left(p^{A}, p^{B}\right)=(1,1) \\ \{0,0\} & \text { if }\left(p^{A}, p^{B}\right)=(2,1) \\ \{0,0\} & \text { if }\left(p^{A}, p^{B}\right)=(1,2) \\ \{0,0\} & \text { if }\left(p^{A}, p^{B}\right)=(2,2)\end{cases}
$$

Now, we have to go back to terrorists' beliefs. Hence

- when $\gamma \in\left[0, \frac{1}{12}\right]$ then $\mu^{2,1 \mid 1,1}=\mu^{1,2 \mid 1,1}=(1-\pi) \pi$ which means that $\mu^{2,1 \mid 1,1}=\mu^{1,2 \mid 1,1}$ is satisfied. 
- when $\gamma \in\left[\frac{1}{12}, \frac{2}{15}\right]$ then $\mu^{2,1 \mid 0,0}=\frac{(1-\pi) \pi}{(1-\pi) \pi+(1-\pi)^{2}}$ and $\mu^{1,2 \mid 0,0}=0$ which means that $\mu^{2,1 \mid 0,0} \leq \mu^{1,2 \mid 0,0}$ is not satisfied.

- when $\gamma \in\left[\frac{2}{15}, \frac{1}{6}\right]$ then $\mu^{2,1 \mid 1,1}=\mu^{1,2 \mid 1,1}=0$ which means that $\mu^{2,1 \mid 1,1}=\mu^{1,2 \mid 1,1}$ is satisfied. Moreover $\mu^{2,1 \mid 0,0}=\mu^{1,2 \mid 0,0}=\frac{(1-\pi) \pi}{2(1-\pi) \pi+(1-\pi)^{2}}$ which means that $\mu^{2,1 \mid 0,0}=\mu^{1,2 \mid 0,0}$ is satisfied

- when $\gamma \in\left[\frac{1}{6}, 1\right]$ then $\mu^{2,1 \mid 0,0}=\mu^{1,2 \mid 0,0}=(1-\pi) \pi$ which means that $\mu^{2,1 \mid 0,0}=\mu^{1,2 \mid 0,0}$ is satisfied.

15. Suppose

$$
\left\{\begin{array}{c}
\mu^{2,1 \mid 0,0} \leq \mu^{1,2 \mid 0,0} \\
\mu^{2,1 \mid 1,0} \leq \frac{15 \mu^{1,2 \mid 1,0}+10}{14} \\
\mu^{2,1 \mid 0,1} \geq \frac{14 \mu^{1,2 \mid 0,1}-10}{15} \\
\mu^{2,1 \mid 1,1} \leq \mu^{1,2 \mid 1,1}
\end{array}\right.
$$

Following the scheme of point 1, we conclude that in these beliefs regions there are the following possible $\mathrm{PBE}$

(a) i. if $\gamma \in\left[0, \frac{1}{12}\right]$

$$
\left\{d^{A *}\left(p^{A}, p^{B}\right), d^{B *}\left(p^{A}, p^{B}\right)\right\}= \begin{cases}\{1,1\} & \text { if }\left(p^{A}, p^{B}\right)=(1,1) \\ \{1,0\} & \text { if }\left(p^{A}, p^{B}\right)=(2,1) \\ \{1,1\} & \text { if }\left(p^{A}, p^{B}\right)=(1,2) \\ \{1,1\} & \text { if }\left(p^{A}, p^{B}\right)=(2,2)\end{cases}
$$

ii. if $\gamma \in\left[\frac{1}{12}, \frac{2}{15}\right]$

$$
\left\{d^{A *}\left(p^{A}, p^{B}\right), d^{B *}\left(p^{A}, p^{B}\right)\right\}= \begin{cases}\{1,1\} & \text { if }\left(p^{A}, p^{B}\right)=(1,1) \\ \{1,0\} & \text { if }\left(p^{A}, p^{B}\right)=(2,1) \\ \{1,1\} & \text { if }\left(p^{A}, p^{B}\right)=(1,2) \\ \{0,0\} & \text { if }\left(p^{A}, p^{B}\right)=(2,2)\end{cases}
$$

iii. if $\gamma \in\left[\frac{2}{15}, \frac{1}{6}\right]$

$$
\left\{d^{A *}\left(p^{A}, p^{B}\right), d^{B *}\left(p^{A}, p^{B}\right)\right\}= \begin{cases}\{1,1\} & \text { if }\left(p^{A}, p^{B}\right)=(1,1) \\ \{0,0\} & \text { if }\left(p^{A}, p^{B}\right)=(2,1) \\ \{0,0\} & \text { if }\left(p^{A}, p^{B}\right)=(1,2) \\ \{0,0\} & \text { if }\left(p^{A}, p^{B}\right)=(2,2)\end{cases}
$$

iv. if $\gamma \in\left[\frac{1}{6}, 1\right]$

$$
\left\{d^{A *}\left(p^{A}, p^{B}\right), d^{B *}\left(p^{A}, p^{B}\right)\right\}= \begin{cases}\{0,0\} & \text { if }\left(p^{A}, p^{B}\right)=(1,1) \\ \{0,0\} & \text { if }\left(p^{A}, p^{B}\right)=(2,1) \\ \{0,0\} & \text { if }\left(p^{A}, p^{B}\right)=(1,2) \\ \{0,0\} & \text { if }\left(p^{A}, p^{B}\right)=(2,2)\end{cases}
$$

Now, we have to go back to terrorists' beliefs. Hence 
- when $\gamma \in\left[0, \frac{1}{12}\right]$ then $\mu^{2,1 \mid 1,0}=1$ and $\mu^{1,2 \mid 1,0}=0$ which means that $\mu^{2,1 \mid 1,0} \leq \frac{15 \mu^{1,2 \mid 1,0}+10}{14}$ is impossible.

- when $\gamma \in\left[\frac{1}{12}, \frac{2}{15}\right]$ then $\mu^{2,1 \mid 1,0}=1$ and $\mu^{1,2 \mid 1,0}=0$ which means that $\mu^{2,1 \mid 1,0} \leq \frac{15 \mu^{1,2 \mid 1,0}+10}{14}$ is impossible.

- when $\gamma \in\left[\frac{2}{15}, \frac{1}{6}\right]$ then $\mu^{2,1 \mid 1,1}=\mu^{1,2 \mid 1,1}=0$ which means that $\mu^{2,1 \mid 1,1}=\mu^{1,2 \mid 1,1}$ is satisfied. Moreover $\mu^{2,1 \mid 0,0}=\mu^{1,2 \mid 0,0}=\frac{(1-\pi) \pi}{2(1-\pi) \pi+(1-\pi)^{2}}$ which means that $\mu^{2,1 \mid 0,0}=\mu^{1,2 \mid 0,0}$ is satisfied.

- When $\gamma \in\left[\frac{1}{6}, 1\right]$ then $\mu^{2,1 \mid 0,0}=\mu^{1,2 \mid 0,0}=(1-\pi) \pi$ which means that $\mu^{2,1 \mid 0,0}=\mu^{1,2 \mid 0,0}$ is satisfied.

16. Suppose

$$
\left\{\begin{array}{c}
\mu^{2,1 \mid 0,0} \leq \mu^{1,2 \mid 0,0} \\
\mu^{2,1 \mid 1,0} \leq \frac{15 \mu^{1,2 \mid 1,0}+10}{14} \\
\mu^{2,1 \mid 0,1} \leq \frac{14 \mu^{1,2 \mid 0,1}-10}{15} \\
\mu^{2,1 \mid 1,1} \leq \mu^{1,2 \mid 1,1}
\end{array}\right.
$$

Following the scheme of point 1 , we conclude that in these beliefs regions there are the following possible PBE

(a) i. if $\gamma \in\left[0, \frac{1}{12}\right]$

$$
\left\{d^{A *}\left(p^{A}, p^{B}\right), d^{B *}\left(p^{A}, p^{B}\right)\right\}= \begin{cases}\{1,1\} & \text { if }\left(p^{A}, p^{B}\right)=(1,1) \\ \{1,1\} & \text { if }\left(p^{A}, p^{B}\right)=(2,1) \\ \{1,1\} & \text { if }\left(p^{A}, p^{B}\right)=(1,2) \\ \{1,1\} & \text { if }\left(p^{A}, p^{B}\right)=(2,2)\end{cases}
$$

ii. if $\gamma \in\left[\frac{1}{12}, \frac{2}{15}\right]$

$$
\left\{d^{A *}\left(p^{A}, p^{B}\right), d^{B *}\left(p^{A}, p^{B}\right)\right\}= \begin{cases}\{1,1\} & \text { if }\left(p^{A}, p^{B}\right)=(1,1) \\ \{0,0\} & \text { if }\left(p^{A}, p^{B}\right)=(2,1) \\ \{1,1\} & \text { if }\left(p^{A}, p^{B}\right)=(1,2) \\ \{0,0\} & \text { if }\left(p^{A}, p^{B}\right)=(2,2)\end{cases}
$$

iii. if $\gamma \in\left[\frac{2}{15}, \frac{1}{6}\right]$

$$
\left\{d^{A *}\left(p^{A}, p^{B}\right), d^{B *}\left(p^{A}, p^{B}\right)\right\}= \begin{cases}\{1,1\} & \text { if }\left(p^{A}, p^{B}\right)=(1,1) \\ \{0,0\} & \text { if }\left(p^{A}, p^{B}\right)=(2,1) \\ \{0,0\} & \text { if }\left(p^{A}, p^{B}\right)=(1,2) \\ \{0,0\} & \text { if }\left(p^{A}, p^{B}\right)=(2,2)\end{cases}
$$

iv. if $\gamma \in\left[\frac{1}{6}, 1\right]$

$$
\left\{d^{A *}\left(p^{A}, p^{B}\right), d^{B *}\left(p^{A}, p^{B}\right)\right\}= \begin{cases}\{0,0\} & \text { if }\left(p^{A}, p^{B}\right)=(1,1) \\ \{0,0\} & \text { if }\left(p^{A}, p^{B}\right)=(2,1) \\ \{0,0\} & \text { if }\left(p^{A}, p^{B}\right)=(1,2) \\ \{0,0\} & \text { if }\left(p^{A}, p^{B}\right)=(2,2)\end{cases}
$$


Now, we have to go back to terrorists' beliefs. Hence

- when $\gamma \in\left[0, \frac{1}{12}\right]$ then $\mu^{2,1 \mid 1,1}=\mu^{1,2 \mid 1,1}=(1-\pi) \pi$ which means that $\mu^{2,1 \mid 1,1}=\mu^{1,2 \mid 1,1}$ is satisfied.

- when $\gamma \in\left[\frac{1}{12}, \frac{2}{15}\right]$ then $\mu^{2,1 \mid 0,0}=\frac{(1-\pi) \pi}{(1-\pi) \pi+(1-\pi)^{2}}$ and $\mu^{1,2 \mid 0,0}=0$ which means that $\mu^{2,1 \mid 0,0} \leq \mu^{1,2 \mid 0,0}$ is not satisfied.

- when $\gamma \in\left[\frac{2}{15}, \frac{1}{6}\right]$ then $\mu^{2,1 \mid 1,1}=\mu^{1,2 \mid 1,1}=0$ which means that $\mu^{2,1 \mid 1,1}=\mu^{1,2 \mid 1,1}$ is satisfied. Moreover $\mu^{2,1 \mid 0,0}=\mu^{1,2 \mid 0,0}=\frac{(1-\pi) \pi}{2(1-\pi) \pi+(1-\pi)^{2}}$ which means that $\mu^{2,1 \mid 0,0}=\mu^{1,2 \mid 0,0}$ is satisfied.

- when $\gamma \in\left[\frac{1}{6}, 1\right]$ then $\mu^{2,1 \mid 0,0}=\mu^{1,2 \mid 0,0}=(1-\pi) \pi$ which means that $\mu^{2,1 \mid 0,0}=\mu^{1,2 \mid 0,0}$ is satisfied.

We sum up the previous results in the following proposition

Proposition 11 1. When $\gamma \in\left[0, \frac{1}{12}\right]$ there is a PBE such that

$$
\begin{gathered}
\left\{d^{A *}\left(p^{A}, p^{B}\right), d^{B *}\left(p^{A}, p^{B}\right)\right\} \in\left\{\begin{array}{l}
\{1,1\} \quad \text { if }\left(p^{A}, p^{B}\right)=(1,1) \\
\{1,1\} \quad \text { if }\left(p^{A}, p^{B}\right)=(2,1) \\
\{1,1\} \quad \text { if }\left(p^{A}, p^{B}\right)=(1,2) \\
\{1,1\} \quad \text { if }\left(p^{A}, p^{B}\right)=(2,2)
\end{array}\right. \\
\left\{\begin{aligned}
\mu^{2,1 \mid 0,0} \gtrless \mu^{1,2 \mid 0,0} \\
\mu^{2,1 \mid 1,0} \gtrless \frac{15 \mu^{1,2 \mid 1,0}+10}{14} \\
\mu^{2,1 \mid 0,1} \gtrless \frac{14 \mu^{1,2 \mid 0,1}-10}{15} \\
\mu^{2,1 \mid 1,1}=\mu^{1,2 \mid 1,1}=(1-\pi) \pi
\end{aligned}\right. \\
a^{*}\left(d^{A}, d^{B}\right) \in\left\{\begin{aligned}
{[0,1] } & \left(d^{A}, d^{B}\right)=(0,0) \\
{[0,1] } & \left(d^{A}, d^{B}\right)=(1,0) \\
{[0,1] } & \left(d^{A}, d^{B}\right)=(0,1) \\
{[0,1] } & \left(d^{A}, d^{B}\right)=(1,1)
\end{aligned}\right.
\end{gathered}
$$

2. When $\gamma \in\left[\frac{1}{12}, \frac{2}{15}\right]$ there is a PBE such that

$$
\begin{gathered}
\left\{d^{A *}\left(p^{A}, p^{B}\right), d^{B *}\left(p^{A}, p^{B}\right)\right\}=\left\{\begin{array}{l}
\{(1,1)\} \quad \text { if }\left(p^{A}, p^{B}\right)=(1,1) \\
\{(1,1)\} \quad \text { if }\left(p^{A}, p^{B}\right)=(2,1) \\
\{(1,1)\} \quad \text { if }\left(p^{A}, p^{B}\right)=(1,2) \\
\{(0,0)\} \quad \text { if }\left(p^{A}, p^{B}\right)=(2,2)
\end{array}\right. \\
\left\{\begin{aligned}
\mu^{2,1 \mid 0,0}=\mu^{1,2 \mid 0,0}=0 \\
\mu^{2,1 \mid 1,0} \leq \frac{15 \mu^{1,2 \mid 1,0}+10}{14} \\
\mu^{2,1 \mid 0,1} \geq \frac{14 \mu^{1,2 \mid 0,1}-10}{15} \\
\mu^{2,1 \mid 1,1}=\mu^{1,2 \mid 1,1}=\frac{(1-\pi) \pi}{\pi^{2}+2(1-\pi) \pi}
\end{aligned}\right. \\
a^{*}\left(d^{A}, d^{B}\right) \in\left\{\begin{array}{cc}
{[0,1]} & \left(d^{A}, d^{B}\right)=(0,0) \\
\{0\} & \left(d^{A}, d^{B}\right)=(1,0) \\
\{1\} & \left(d^{A}, d^{B}\right)=(0,1) \\
{[0,1]} & \left(d^{A}, d^{B}\right)=(1,1)
\end{array}\right.
\end{gathered}
$$


3. When $\gamma \in\left[\frac{2}{15}, \frac{1}{6}\right]$ there is a PBE such that

$$
\begin{aligned}
& \left\{d^{A *}\left(p^{A}, p^{B}\right), d^{B *}\left(p^{A}, p^{B}\right)\right\}= \begin{cases}\{1,1\} & \text { if }\left(p^{A}, p^{B}\right)=(1,1) \\
\{0,0\} & \text { if }\left(p^{A}, p^{B}\right)=(2,1) \\
\{0,0\} & \text { if }\left(p^{A}, p^{B}\right)=(1,2) \\
\{0,0\} & \text { if }\left(p^{A}, p^{B}\right)=(2,2)\end{cases} \\
& \left\{\begin{array}{c}
\mu^{2,1 \mid 0,0}=\mu^{1,2 \mid 0,0}=\frac{(1-\pi) \pi}{2(1-\pi) \pi+(1-\pi)^{2}} \\
\mu^{2,1 \mid 1,0} \lessgtr \frac{15 \mu^{1,2 \mid 1,0}+10}{14} \\
\mu^{2,1 \mid 0,1} \lessgtr \frac{14 \mu^{1,2 \mid 0,1}-10}{15} \\
0=\mu^{2,1 \mid 1,1}=\mu^{1,2 \mid 1,1}
\end{array}\right. \\
& a^{*}\left(d^{A}, d^{B}\right) \in \begin{cases}{[0,1]} & \left(d^{A}, d^{B}\right)=(0,0) \\
{[0,1]} & \left(d^{A}, d^{B}\right)=(1,0) \\
{[0,1]} & \left(d^{A}, d^{B}\right)=(0,1) \\
{[0,1]} & \left(d^{A}, d^{B}\right)=(1,1)\end{cases}
\end{aligned}
$$

4. when $\gamma \in\left[\frac{1}{6}, 1\right]$ there is a PBE such that

$$
\begin{gathered}
\left\{d^{A *}\left(p^{A}, p^{B}\right), d^{B *}\left(p^{A}, p^{B}\right)\right\}=\left\{\begin{array}{l}
\{0,0\} \quad \text { if }\left(p^{A}, p^{B}\right)=(1,1) \\
\{0,0\} \quad \text { if }\left(p^{A}, p^{B}\right)=(2,1) \\
\{0,0\} \quad \text { if }\left(p^{A}, p^{B}\right)=(1,2) \\
\{0,0\} \quad \text { if }\left(p^{A}, p^{B}\right)=(2,2)
\end{array}\right. \\
\left\{\begin{array}{r}
\mu^{2,1 \mid 0,0}=\mu^{1,2 \mid 0,0}=(1-\pi) \pi \\
\mu^{2,1 \mid 1,0} \lessgtr \frac{15 \mu^{1,2 \mid 1,0}+10}{14} \\
\mu^{2,1 \mid 0,1} \lessgtr \frac{14 \mu^{1,2 \mid 0,1}-10}{15} \\
\mu^{2,1 \mid 1,1} \lessgtr \mu^{1,2 \mid 1,1}
\end{array}\right. \\
a^{*}\left(d^{A}, d^{B}\right) \in\left\{\begin{array}{rr}
{[0,1]} & \left(d^{A}, d^{B}\right)=(0,0) \\
{[0,1]} & \left(d^{A}, d^{B}\right)=(1,0) \\
{[0,1]} & \left(d^{A}, d^{B}\right)=(0,1) \\
{[0,1]} & \left(d^{A}, d^{B}\right)=(1,1)
\end{array}\right.
\end{gathered}
$$

